\title{
Summary Data on Cooling Water Use at Utilities and Nonutilities
}
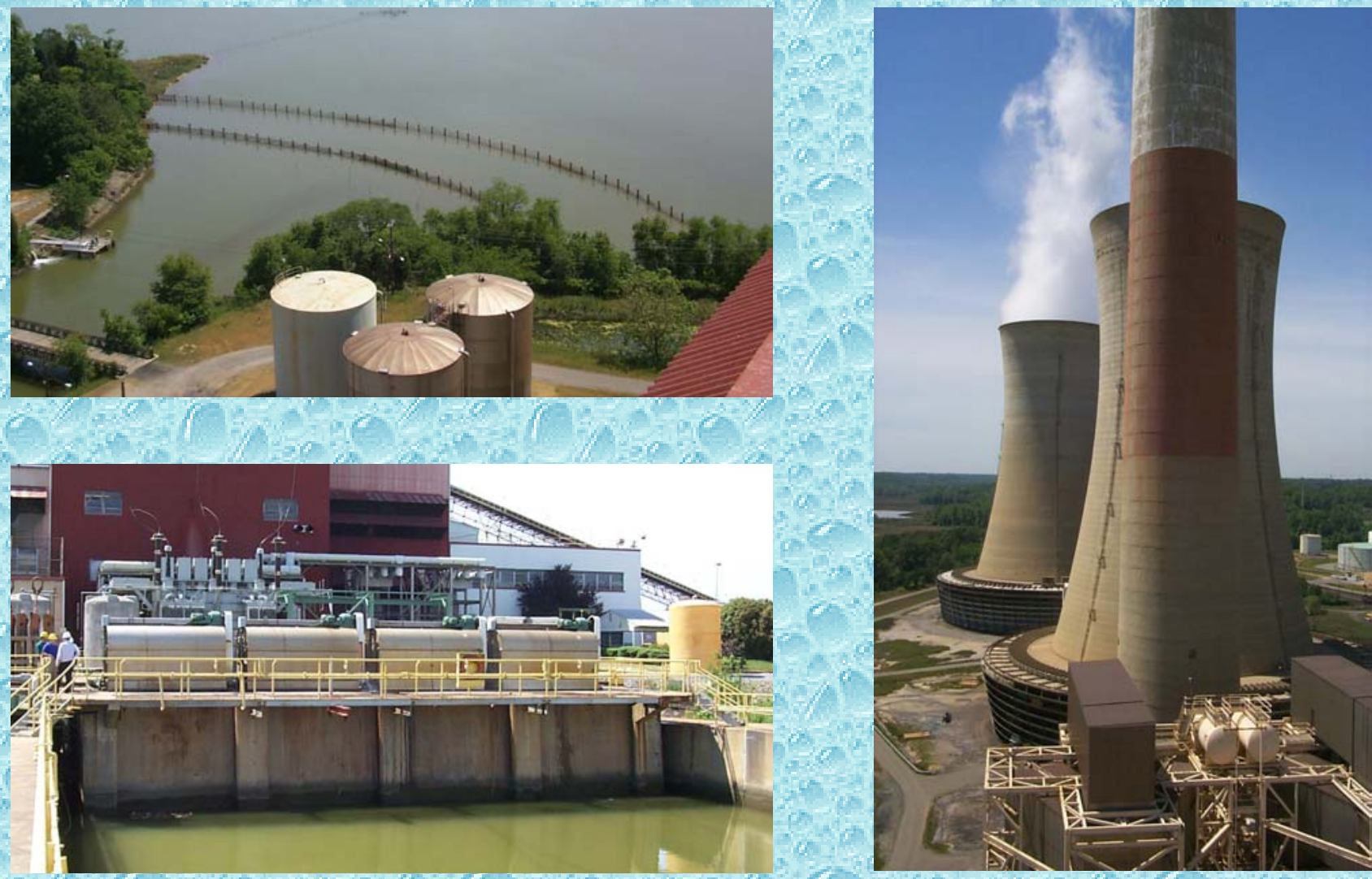

Prepared for U.S. Department of Energy Office of Fossil Energy and Office of Policy and International Affairs under Contract W-31-109-Eng-38

Prepared by Markus G. Puder and John A. Veil Argonne National Laboratory 


\title{
Summary Data on Cooling Water Use at Utilities and Nonutilities
}

\author{
Markus G. Puder and John A. Veil \\ Argonne National Laboratory
}

$\underline{\text { Background }}$

The U.S. Environmental Protection Agency (EPA) is currently developing regulations to implement $\S 316($ b) of the Clean Water Act. Section 316(b) specifies that "any standard established pursuant to section 301 or section 306 of this Act and applicable to a point source shall require that the location, design, construction, and capacity of cooling water intake structures reflect the best technology available for minimizing adverse environmental impact." The EPA initially planned to propose regulations by the summer of 1999; however, it has postponed that date indefinitely.

In the fall of 1998, the U.S. Department of Energy (DOE), recognizing the potential of the proposed $\S 316$ (b) regulations to cause economic impacts on the electric power industry, commissioned Argonne National Laboratory (Argonne) to collect information on cooling water use patterns at utility and nonutility electricity generating facilities. One purpose of the project was to estimate whether the economic impacts of the final $\$ 316(\mathrm{~b})$ regulations would cause some companies to shut down generating units or to switch units to part-time or seasonal operation rather than retrofit existing units with new compliant technologies. If such reductions in generating capacity were clustered in particular states or regions, electricity reliability problems could occur.

Argonne began collecting information on the subset of electric generating facilities that use once-through cooling systems. These are the facilities that potentially could be affected by the $\$ 316(\mathrm{~b})$ regulations. Extensive discussion took place between DOE and Argonne about the scope of the study. A tentative project scope was described at an April 1999 conference on $\$ 316($ b) issues sponsored by the Electric Power Research Institute and DOE (Veil 1999). After hearing remarks by an EPA representative at the same conference, however, it became apparent that a study on electricity reliability effects of the $\$ 316(\mathrm{~b})$ regulations would not be appropriate or necessary. Consequently, the DOE project managers (Debra Littleton and David Moses) directed Argonne to discontinue work on the reliability study and to compile the data that had been collected into a data summary report. This document represents a compilation of the information Argonne has collected to date on the following:

- Cooling water use at utilities,

- Cooling water use at nonutilities,

- $\quad$ Estimated capital costs for retrofitting cooling towers at plants presently using oncethrough cooling,

- $\quad$ Operations and maintenance (O\&M) costs for retrofitted cooling towers,

- $\quad$ Size of the energy penalty experienced by plants converting from once-through cooling 
systems to cooling towers, and

- Amount of outage time needed to retrofit cooling towers at a plant presently using oncethrough cooling.

Each of these topics is discussed in the following sections.

\section{$\underline{\text { Cooling Water Use at Utilities }}$}

For 1997, DOE's Energy Information Administration (EIA) reported that U.S. generators produced 778,513 megawatts (MW) of electricity. Utilities produced 711,889 MW, whereas $74,021 \mathrm{MW}$ was generated by nonutility sources. The EIA noted that its methodology does not permit direct summation of the utility and nonutility totals (EIA 1998a). The Edison Electric Institute (EEI) reported that of the utility total, approximately $584,000 \mathrm{MW}$ can be attributed to steam electric plants that require a cooling cycle. Of these, about $44 \%$ utilize once-through cooling systems, and another $5 \%$ utilize a combination of once-through cooling and closed-cycle cooling (EEI 1996).

Argonne attempted to identify all utility units that use just once-through cooling systems or use once-through systems in conjunction with other cooling systems like helper cooling towers. Information on these units was stored in a Microsoft Access database. A printout of the database is attached as Appendix 1. The database contains 12 data fields for 1,321 different generating units representing $288,633 \mathrm{MW}$. The data are arranged alphabetically by plant name. "Plant Name" provides the name of the plant. Two-letter state abbreviations appear after those plants that have the same name as plants in other states. "Utility Name" specifies the name of the operating utility company. "State" denotes the state in which the plant is located. "Utility/Plant/Unit ID" furnishes the respective utility, plant, and unit identification numbers assigned by the EIA. "MW" provides the plant's nameplate unit capacity in megawatts. "Status" explains whether the plant is in commercial operation or on standby, shut down, or in economic reserve. "Year" specifies the actual or projected year of commercial operation. "Fuel" denotes whether the plant's primary fuel is coal, gas, oil, refuse-derived, uranium, or wood. "Water" furnishes information pertaining to the type of cooling water used at the plant (i.e., brackish, fresh, ground, municipal, saline, or unknown). "Cooling System" explains whether the plant uses a once-through, closed cycle, combination or mixed mode of cooling.

The data were extracted primarily from the latest version of the EEI Power Statistics Data Base (EEI 1996), which includes data compiled through December 1995. The unit, plant, and utility identification numbers were obtained from the EIA's Form EIA-767 database. Identification numbers were not included for certain plants and utilities because Form EIA-767 does not provide data for U.S. entities (Guam, Puerto Rico, and Virgin Islands) other than the 50 states. Identification numbers could not be secured for a few additional units that are reported in the EEI Power Statistics Database. These facilities are either very small or nonoperational.

Cooling Water Use at Nonutilities 
No comprehensive data were previously available on the types of cooling water systems used by nonutility facilities. Therefore, any cooling water data collected for the nonutility sector had to be conducted by directly contacting facilities. Approximately 2,000 nonutility electricity generating facilities exist in the United States, far too many to be contacted individually (EIA 1998b). The generating capacity of these plants ranges from 1 to 1,849 MW. Argonne determined that $56 \%$ of the total nonutility capacity $(41,494 \mathrm{MW})$ could be captured by examining only the 123 facilities generating at least $150 \mathrm{MW}$. Each of these facilities was contacted, and the results were entered into a database. Argonne obtained cooling water use information from an additional 58 waste-to-energy facilities, which represent about 2,200 MW and entered it into another database. Both these databases have been included in a manuscript submitted for publication in a scientific journal in June 1999 (Veil et al. 1999). A copy of the manuscript is attached as Appendix 2.

Only a relatively small portion of nonutility power sources utilize once-through cooling. The percentage of the generating capacity using once-through cooling ranges from 11 to $17 \%$ in the two subsets of the nonutility population that were studied. A conservative estimate of the total once-through nonutility power generation yields $15,372 \mathrm{MW}$, which is equivalent to only $6 \%$ of the utility capacity employing once-through cooling.

Estimated Capital Costs for Retrofitting Cooling Towers at Plants Presently Using OnceThrough Cooling

If Argonne were asked to estimate the current capital costs for retrofitting cooling towers at plants presently using once-through cooling, it would use the cost rates developed in a previous Argonne study (Veil 1993) and scale up the report's 1992 dollars to 1998 dollars by a factor of 1.19, as indicated by the Construction Cost Index (Engineering News Record 1999). The resulting cost rates would be $\$ 125 / \mathrm{kilowatt}(\mathrm{kw})$ to $\$ 128 / \mathrm{kw}$ for fossil-fuel plants and $\$ 203 / \mathrm{kw}$ to $\$ 239 / \mathrm{kw}$ for nuclear plants.

\section{Operations and Maintenance (O\&M) Costs for Retrofitted Cooling Towers}

Stone and Webster (1992) has estimated that O\&M costs of cooling towers are approximately $0.7 \%$ of capital costs. Such a small percentage easily falls within the margin of error of our cost estimate. Consequently, O\&M costs are considered to be negligible in relation to capital costs.

Size of the Energy Penalty Experienced by Plants Converting from Once-Through Cooling Systems to Cooling Towers

When a generating unit is switched from once-through cooling to a cooling tower system, the net amount of energy produced by the unit is diminished by an amount known as the energy penalty. The energy penalty is caused by a reduction in turbine back pressure and the need to operate more recirculating pumps to lift cooling water into the cooling tower structure. Veil et al. (1993) surveyed literature values of the energy penalty and found a range of 1.0 to $5.8 \%$. 
Most values fell within the range of 1.5 to $2.5 \%$ for fossil-fuel plants and 2.0 to $3.0 \%$ for nuclear plants.

The Amount of Outage Time Needed to Retrofit Cooling Towers at a Plant Presently Using Once-Through Cooling

Argonne contacted representatives from cooling tower manufacturers, construction engineering firms, and utilities and learned that it is impossible to generalize the length of outage needed to convert a once-through cooling system to a cooling tower. If a plant has adequate land area to build a tower while the plant is in service, it may be possible to connect the tower to the cooling system during a scheduled one- to two-month outage. In other cases, the plant layout does not allow simultaneous construction of the new system, and the needed outage could be as long as one year.

\section{$\underline{\text { Final Notes }}$}

Some of the data reported here are compilations of existing information (e.g., utility cooling water use information); other data are completely new (e.g., nonutility cooling water use information, time needed for retrofit). Although the proposed study on electricity reliability has been postponed indefinitely, the information included in this summary report should be useful for the ongoing $\$ 316(\mathrm{~b})$ rulemaking and for future analyses.

\section{$\underline{\text { References }}$}

EEI, 1996, Environmental Directory of US Powerplants, Edison Electric Institute, Washington, DC.

EIA, 1998a, Electric Power Annual 1997, Volume II, U.S. Department of Energy, Energy Information Administration, DOE/EIA-0348(97)/2, October.

EIA, 1998b, Inventory of Power Plants in the United States As of January 1, 1998, U.S. Department of Energy, Energy Information Administration, DOE/EIA-0095(98), December.

Engineering News Record, 1999, website at [http://www.enr.com/cost/costcci.asp]

Stone and Webster, 1992, Evaluation of the Potential Costs and Environmental Impacts of Retrofitting Cooling Towers on Existing Steam Electric Power Plants That Have Obtained Variances under Section 316(a) of the Clean Water Act, prepared for Edison Electric Institute, Washington, DC.

Veil, J.A., 1993, Impact on the Steam Electric Power Industry of Deleting Section 316(a) of the Clean Water Act: Capital Costs, ANL/EAIS-4, Argonne National Laboratory, Argonne, IL, January. 
Veil, J.A., J.C. VanKuiken, S. Folga, and J.L. Gillette, 1993, Impact on the Steam Electric Power Industry of Deleting Section 316(a) of the Clean Water Act: Energy and Environmental Impacts, ANL/EAIS-5, Argonne National Laboratory, Argonne, IL, January.

Veil, J.A., 1999, "Potential Impacts of 316(b) Regulatory Controls on Economics, Electricity Reliability, and the Environment," in Proceedings of the Power Generation Impacts on Aquatic Resources Conference, sponsored by the Electric Power Research Institute and the U.S. Department of Energy, April 12-15, 1999, Atlanta, GA.

Veil, J.A., and M.G. Puder, D.J. Littleton, and D.O. Moses, 1999, "Cooling Water Use Patterns at U.S. Nonutility Electric Generating Stations," manuscript submitted for publication in Environmental Science \& Policy, June. 
Appendix 1 - Electric Utilities and Their Cooling Systems (printout of Microsoft Access Database) 


\section{Electric Utilities and Their Cooling Systems}

\begin{tabular}{|c|c|c|c|c|c|c|c|c|c|c|c|}
\hline Plant Name & Utility Name & State & Utility/P & $n t / U n i$ & $I D$ & $M W$ & Status & Year & Fuel & Water & Cooling System \\
\hline ADVANCE & $\begin{array}{l}\text { WOLVERINE PWR } \\
\text { SUPPLY COOP }\end{array}$ & MI & 20910 & 1869 & 2 & 7.5 & Operational & 1953 & COAL & FRESH & ONCE THROUGH \\
\hline ADVANCE & $\begin{array}{l}\text { WOLVERINE PWR } \\
\text { SUPPLY COOP }\end{array}$ & MI & 20910 & 1869 & 3 & 22 & Operational & 1967 & COAL & FRESH & ONCE THROUGH \\
\hline ADVANCE & $\begin{array}{l}\text { WOLVERINE PWR } \\
\text { SUPPLY COOP }\end{array}$ & MI & 20910 & 1869 & 1 & 7.5 & Operational & 1953 & COAL & FRESH & ONCE THROUGH \\
\hline AGUIRRE & $\begin{array}{l}\text { PUERTO RICO ELEC } \\
\text { PWR AUTH }\end{array}$ & PR & 15497 & & 1 & 485 & Operational & 1975 & OIL & SALINE & ONCE THROUGH \\
\hline AGUIRRE & $\begin{array}{l}\text { PUERTO RICO ELEC } \\
\text { PWR AUTH }\end{array}$ & PR & 15497 & & 2 & 485 & Operational & 1975 & OIL & SALINE & ONCE THROUGH \\
\hline ALAMITOS & $\begin{array}{l}\text { SOUTHERN CALIF } \\
\text { EDISON CO }\end{array}$ & $\mathrm{CA}$ & 17609 & 315 & 3 & 333 & Operational & 1961 & GAS & SALINE & ONCE THROUGH \\
\hline ALAMITOS & $\begin{array}{l}\text { SOUTHERN CALIF } \\
\text { EDISON CO }\end{array}$ & $\mathrm{CA}$ & 17609 & 315 & 4 & 333 & Operational & 1962 & GAS & SALINE & ONCE THROUGH \\
\hline ALAMITOS & $\begin{array}{l}\text { SOUTHERN CALIF } \\
\text { EDISON CO }\end{array}$ & $\mathrm{CA}$ & 17609 & 315 & 6 & 495 & Operational & 1966 & GAS & SALINE & ONCE THROUGH \\
\hline ALAMITOS & $\begin{array}{l}\text { SOUTHERN CALIF } \\
\text { EDISON CO }\end{array}$ & $\mathrm{CA}$ & 17609 & 315 & 2 & 163 & Operational & 1957 & GAS & SALINE & ONCE THROUGH \\
\hline ALAMITOS & $\begin{array}{l}\text { SOUTHERN CALIF } \\
\text { EDISON CO }\end{array}$ & $\mathrm{CA}$ & 17609 & 315 & 1 & 163 & Operational & 1956 & GAS & SALINE & ONCE THROUGH \\
\hline ALAMITOS & $\begin{array}{l}\text { SOUTHERN CALIF } \\
\text { EDISON CO }\end{array}$ & $\mathrm{CA}$ & 17609 & 315 & 5 & 495 & Operational & 1966 & GAS & SALINE & ONCE THROUGH \\
\hline ALBANY & $\begin{array}{l}\text { NIAGARA MOHAWK } \\
\text { POWER CORP }\end{array}$ & NY & 13573 & 2539 & 1 & 100 & Operational & 1952 & GAS & FRESH & ONCE THROUGH \\
\hline
\end{tabular}




\begin{tabular}{|c|c|c|c|c|c|c|c|c|c|c|c|}
\hline Plant Name & Utility Name & State & Utility/P & $n t / U n i$ & $I D$ & $M W$ & Status & Year & Fuel & Water & Cooling System \\
\hline ALBANY & $\begin{array}{l}\text { NIAGARA MOHAWK } \\
\text { POWER CORP }\end{array}$ & NY & 13573 & 2539 & 2 & 100 & Operational & 1952 & GAS & FRESH & ONCE THROUGH \\
\hline ALBANY & $\begin{array}{l}\text { NIAGARA MOHAWK } \\
\text { POWER CORP }\end{array}$ & NY & 13573 & 2539 & 3 & 100 & Operational & 1953 & GAS & FRESH & ONCE THROUGH \\
\hline ALBANY & $\begin{array}{l}\text { NIAGARA MOHAWK } \\
\text { POWER CORP }\end{array}$ & NY & 13573 & 2539 & 4 & 100 & Operational & 1954 & OIL & FRESH & ONCE THROUGH \\
\hline ALBRIGHT & $\begin{array}{l}\text { MONONGAHELA } \\
\text { POWER CO }\end{array}$ & WV & 12796 & 3942 & 2 & 69 & Operational & 1952 & COAL & FRESH & $\begin{array}{l}\text { MIXED MODE (ONCE } \\
\text { THROUGH/COOLING TOWER) }\end{array}$ \\
\hline ALBRIGHT & $\begin{array}{l}\text { MONONGAHELA } \\
\text { POWER CO }\end{array}$ & WV & 12796 & 3942 & 3 & 140 & Operational & 1954 & COAL & FRESH & $\begin{array}{l}\text { MIXED MODE (ONCE } \\
\text { THROUGH/COOLING TOWER) }\end{array}$ \\
\hline ALBRIGHT & $\begin{array}{l}\text { MONONGAHELA } \\
\text { POWER CO }\end{array}$ & WV & 12796 & 3942 & 1 & 69 & Operational & 1952 & COAL & FRESH & $\begin{array}{l}\text { MIXED MODE (ONCE } \\
\text { THROUGH/COOLING TOWER) }\end{array}$ \\
\hline ALLEN & DUKE POWER CO & $\mathrm{NC}$ & 5416 & 2718 & 5 & 275 & Operational & 1961 & COAL & FRESH & ONCE THROUGH \\
\hline ALLEN & DUKE POWER CO & $\mathrm{NC}$ & 5416 & 2718 & 1 & 165 & Operational & 1957 & COAL & FRESH & ONCE THROUGH \\
\hline ALLEN & DUKE POWER CO & NC & 5416 & 2718 & 2 & 165 & Operational & 1957 & COAL & FRESH & ONCE THROUGH \\
\hline ALLEN & DUKE POWER CO & $\mathrm{NC}$ & 5416 & 2718 & 3 & 275 & Operational & 1959 & COAL & FRESH & ONCE THROUGH \\
\hline ALLEN & DUKE POWER CO & $\mathrm{NC}$ & 5416 & 2718 & 4 & 275 & Operational & 1960 & COAL & FRESH & ONCE THROUGH \\
\hline ALLEN S KING & $\begin{array}{l}\text { NORTHERN STATES } \\
\text { POWER CO }\end{array}$ & $\mathrm{MN}$ & 13781 & 1915 & 1 & 598 & Operational & 1968 & COAL & FRESH & $\begin{array}{l}\text { COMBINATION (ONCE } \\
\text { THROUGH/HELPER TOWER) }\end{array}$ \\
\hline ALMA & $\begin{array}{l}\text { DAIRYLAND POWER } \\
\text { COOP }\end{array}$ & WI & 4716 & 4140 & 2 & 15 & Operational & 1947 & COAL & FRESH & ONCE THROUGH \\
\hline ALMA & $\begin{array}{l}\text { DAIRYLAND POWER } \\
\text { COOP }\end{array}$ & WI & 4716 & 4140 & 3 & 15 & Operational & 1951 & COAL & FRESH & ONCE THROUGH \\
\hline
\end{tabular}




\begin{tabular}{|c|c|c|c|c|c|c|c|c|c|c|c|}
\hline Plant Name & Utility Name & State & Utility/P & $n t / U n i$ & $I D$ & $M W$ & Status & Year & Fuel & Water & Cooling System \\
\hline ALMA & $\begin{array}{l}\text { DAIRYLAND POWER } \\
\text { COOP }\end{array}$ & WI & 4716 & 4140 & 4 & 54.4 & Operational & 1957 & COAL & FRESH & ONCE THROUGH \\
\hline ALMA & $\begin{array}{l}\text { DAIRYLAND POWER } \\
\text { COOP }\end{array}$ & WI & 4716 & 4140 & 5 & 81.6 & Operational & 1959 & COAL & FRESH & ONCE THROUGH \\
\hline ALMA & $\begin{array}{l}\text { DAIRYLAND POWER } \\
\text { COOP }\end{array}$ & WI & 4716 & 4140 & 1 & 15 & Operational & 1947 & COAL & FRESH & ONCE THROUGH \\
\hline AM WILLIAMS & $\begin{array}{l}\text { SOUTH CAROLINA } \\
\text { GEN CO }\end{array}$ & SC & 17554 & 3298 & 1 & 633 & Operational & 1973 & COAL & BRACKISH & $\begin{array}{l}\text { COMBINATION (ONCE } \\
\text { THROUGH/HELPER TOWER) }\end{array}$ \\
\hline ANCLOTE & $\begin{array}{l}\text { FLORIDA POWER } \\
\text { CORP }\end{array}$ & $\mathrm{FL}$ & 6455 & 8048 & 1 & 556 & Operational & 1974 & OIL & BRACKISH & $\begin{array}{l}\text { COMBINATION (ONCE } \\
\text { THROUGH/HELPER TOWER) }\end{array}$ \\
\hline ANCLOTE & $\begin{array}{l}\text { FLORIDA POWER } \\
\text { CORP }\end{array}$ & $\mathrm{FL}$ & 6455 & 8048 & 2 & 556 & Operational & 1978 & OIL & BRACKISH & $\begin{array}{l}\text { COMBINATION (ONCE } \\
\text { THROUGH/HELPER TOWER) }\end{array}$ \\
\hline ANDRUS & $\begin{array}{l}\text { MISSISSIPPI } \\
\text { POWER \& LIGHT }\end{array}$ & MS & 12685 & 8054 & 1 & 782 & Operational & 1974 & OIL & FRESH & ONCE THROUGH \\
\hline ANIMAS & $\begin{array}{l}\text { FARMINGTON ELEC } \\
\text { UTIL }\end{array}$ & NM & 6204 & 2465 & 4 & 16.5 & Operational & 1959 & GAS & FRESH & $\begin{array}{l}\text { COMBINATION (ONCE } \\
\text { THROUGH/HELPER TOWER) }\end{array}$ \\
\hline ANIMAS & $\begin{array}{l}\text { FARMINGTON ELEC } \\
\text { UTIL }\end{array}$ & NM & 6204 & 2465 & 3 & 9 & Operational & 1958 & GAS & FRESH & $\begin{array}{l}\text { COMBINATION (ONCE } \\
\text { THROUGH/HELPER TOWER) }\end{array}$ \\
\hline ANIMAS & $\begin{array}{l}\text { FARMINGTON ELEC } \\
\text { UTIL }\end{array}$ & NM & 6204 & 2465 & 1 & 3 & Standby & 1955 & GAS & FRESH & $\begin{array}{l}\text { COMBINATION (ONCE } \\
\text { THROUGH/HELPER TOWER) }\end{array}$ \\
\hline ANIMAS & $\begin{array}{l}\text { FARMINGTON ELEC } \\
\text { UTIL }\end{array}$ & NM & 6204 & 2465 & 2 & 3 & Standby & 1955 & GAS & FRESH & $\begin{array}{l}\text { COMBINATION (ONCE } \\
\text { THROUGH/HELPER TOWER) }\end{array}$ \\
\hline $\begin{array}{l}\text { ARKANSAS } \\
\text { ONE }\end{array}$ & $\begin{array}{l}\text { ENTERGY } \\
\text { OPERATIONS INC }\end{array}$ & $A R$ & 6001 & 8055 & 1 & 903 & Operational & 1974 & UR & FRESH & ONCE THROUGH \\
\hline ARKWRIGHT & $\begin{array}{l}\text { GEORGIA POWER } \\
\text { CO }\end{array}$ & GA & 7140 & 699 & 4 & 49 & Operational & 1948 & COAL & FRESH & ONCE THROUGH \\
\hline ARKWRIGHT & $\begin{array}{l}\text { GEORGIA POWER } \\
\text { CO }\end{array}$ & GA & 7140 & 699 & 3 & 40.3 & Operational & 1943 & COAL & FRESH & ONCE THROUGH \\
\hline
\end{tabular}




\begin{tabular}{|c|c|c|c|c|c|c|c|c|c|c|c|}
\hline Plant Name & Utility Name & State & Utility/Pl & $n t / U n i$ & $I D$ & $M W$ & Status & Year & Fuel & Water & Cooling System \\
\hline ARKWRIGHT & $\begin{array}{l}\text { GEORGIA POWER } \\
\text { CO }\end{array}$ & GA & 7140 & 699 & 2 & 46 & Operational & 1942 & COAL & FRESH & ONCE THROUGH \\
\hline ARKWRIGHT & $\begin{array}{l}\text { GEORGIA POWER } \\
\text { CO }\end{array}$ & GA & 7140 & 699 & 1 & 46 & Operational & 1941 & COAL & FRESH & ONCE THROUGH \\
\hline ARMSTRONG & $\begin{array}{l}\text { WEST PENN } \\
\text { POWER CO }\end{array}$ & PA & 20387 & 3178 & 1 & 163 & Operational & 1958 & COAL & FRESH & ONCE THROUGH \\
\hline ARMSTRONG & $\begin{array}{l}\text { WEST PENN } \\
\text { POWER CO }\end{array}$ & PA & 20387 & 3178 & 2 & 163 & Operational & 1959 & COAL & FRESH & ONCE THROUGH \\
\hline ARTHUR KILL & $\begin{array}{l}\text { CONSOLIDATED } \\
\text { EDISON CO }\end{array}$ & NY & 4226 & 2490 & 2 & 376 & Operational & 1959 & GAS & SALINE & ONCE THROUGH \\
\hline ARTHUR KILL & $\begin{array}{l}\text { CONSOLIDATED } \\
\text { EDISON CO }\end{array}$ & NY & 4226 & 2490 & 3 & 536 & Operational & 1969 & GAS & SALINE & ONCE THROUGH \\
\hline ASHTABULA & $\begin{array}{l}\text { CLEVELAND ELEC } \\
\text { ILLUM CO }\end{array}$ & $\mathrm{OH}$ & 3755 & 2835 & 5 & 256 & Operational & 1958 & COAL & FRESH & ONCE THROUGH \\
\hline ASHTABULA & $\begin{array}{l}\text { CLEVELAND ELEC } \\
\text { ILLUM CO }\end{array}$ & $\mathrm{OH}$ & 3755 & 2835 & 6 & 46 & Operational & 1949 & COAL & FRESH & ONCE THROUGH \\
\hline ASHTABULA & $\begin{array}{l}\text { CLEVELAND ELEC } \\
\text { ILLUM CO }\end{array}$ & $\mathrm{OH}$ & 3755 & 2835 & 7 & 46 & Operational & 1949 & COAL & FRESH & ONCE THROUGH \\
\hline ASHTABULA & $\begin{array}{l}\text { CLEVELAND ELEC } \\
\text { ILLUM CO }\end{array}$ & $\mathrm{OH}$ & 3755 & 2835 & 8 & 46 & Operational & 1948 & COAL & FRESH & ONCE THROUGH \\
\hline ASHTABULA & $\begin{array}{l}\text { CLEVELAND ELEC } \\
\text { ILLUM CO }\end{array}$ & $\mathrm{OH}$ & 3755 & 2835 & 9 & 46 & Operational & 1948 & COAL & FRESH & ONCE THROUGH \\
\hline ASTORIA (NY) & $\begin{array}{l}\text { CONSOLIDATED } \\
\text { EDISON CO }\end{array}$ & NY & 4226 & 8906 & 3 & 376 & Operational & 1958 & GAS & SALINE & ONCE THROUGH \\
\hline ASTORIA (NY) & $\begin{array}{l}\text { CONSOLIDATED } \\
\text { EDISON CO }\end{array}$ & NY & 4226 & 8906 & 5 & 387 & Operational & 1962 & GAS & SALINE & ONCE THROUGH \\
\hline ASTORIA (NY) & $\begin{array}{l}\text { CONSOLIDATED } \\
\text { EDISON CO }\end{array}$ & NY & 4226 & 8906 & 4 & 387 & Operational & 1961 & GAS & SALINE & ONCE THROUGH \\
\hline
\end{tabular}




\begin{tabular}{|c|c|c|c|c|c|c|c|c|c|c|c|}
\hline Plant Name & Utility Name & State & Utility/Pl & $n t / U n i$ & $I D$ & $M W$ & Status & Year & Fuel & Water & Cooling System \\
\hline ATKINSON & $\begin{array}{l}\text { GEORGIA POWER } \\
\text { CO }\end{array}$ & GA & 7140 & 700 & 3 & 63 & Operational & 1945 & GAS & FRESH & ONCE THROUGH \\
\hline ATKINSON & $\begin{array}{l}\text { GEORGIA POWER } \\
\text { CO }\end{array}$ & GA & 7140 & 700 & 4 & 75 & Operational & 1948 & GAS & FRESH & ONCE THROUGH \\
\hline ATKINSON & $\begin{array}{l}\text { GEORGIA POWER } \\
\text { CO }\end{array}$ & GA & 7140 & 700 & 2 & 60 & Operational & 1941 & GAS & FRESH & ONCE THROUGH \\
\hline AVON LAKE & $\begin{array}{l}\text { CLEVELAND ELEC } \\
\text { ILLUM CO }\end{array}$ & $\mathrm{OH}$ & 3755 & 2336 & 7 & 86 & Operational & 1949 & COAL & FRESH & ONCE THROUGH \\
\hline AVON LAKE & $\begin{array}{l}\text { CLEVELAND ELEC } \\
\text { ILLUM CO }\end{array}$ & $\mathrm{OH}$ & 3755 & 2336 & 9 & 680 & Operational & 1970 & COAL & FRESH & ONCE THROUGH \\
\hline AVON LAKE & $\begin{array}{l}\text { CLEVELAND ELEC } \\
\text { ILLUM CO }\end{array}$ & $\mathrm{OH}$ & 3755 & 2336 & 6 & 86 & Operational & 1949 & COAL & FRESH & ONCE THROUGH \\
\hline AVON PARK & $\begin{array}{l}\text { FLORIDA POWER } \\
\text { CORP }\end{array}$ & $\mathrm{FL}$ & 6455 & & 2 & 46 & Standby & 1952 & OIL & FRESH & ONCE THROUGH \\
\hline BAILEY & $\begin{array}{l}\text { ARKANSAS ELEC } \\
\text { COOP CORP }\end{array}$ & $\mathrm{AR}$ & 807 & 202 & 1 & 122 & Operational & 1966 & GAS & FRESH & ONCE THROUGH \\
\hline BAILLY & $\begin{array}{l}\text { NO INDIANA PUBLIC } \\
\text { SERVICE }\end{array}$ & IN & 13756 & 995 & 7 & 194 & Operational & 1962 & COAL & FRESH & ONCE THROUGH \\
\hline BAILLY & $\begin{array}{l}\text { NO INDIANA PUBLIC } \\
\text { SERVICE }\end{array}$ & IN & 13756 & 995 & 8 & 422 & Operational & 1968 & COAL & FRESH & ONCE THROUGH \\
\hline BARNEY DAVIS & $\begin{array}{l}\text { CENTRAL POWER \& } \\
\text { LIGHT CO }\end{array}$ & $\mathrm{TX}$ & 3278 & 4939 & 1 & 353 & Operational & 1974 & GAS & BRACKISH & $\begin{array}{l}\text { COMBINATION (ONCE } \\
\text { THROUGH/HELPER POND) }\end{array}$ \\
\hline BARNEY DAVIS & $\begin{array}{l}\text { CENTRAL POWER \& } \\
\text { LIGHT CO }\end{array}$ & $\mathrm{TX}$ & 3278 & 4939 & 2 & 351 & Operational & 1976 & GAS & BRACKISH & $\begin{array}{l}\text { COMBINATION (ONCE } \\
\text { THROUGH/HELPER POND) }\end{array}$ \\
\hline BARRY & $\begin{array}{l}\text { ALABAMA POWER } \\
\text { CO }\end{array}$ & $\mathrm{AL}$ & 195 & 3 & 4 & 404 & Operational & 1969 & COAL & FRESH & ONCE THROUGH \\
\hline BARRY & $\begin{array}{l}\text { ALABAMA POWER } \\
\text { CO }\end{array}$ & $\mathrm{AL}$ & 195 & 3 & 5 & 789 & Operational & 1971 & COAL & FRESH & ONCE THROUGH \\
\hline
\end{tabular}




\begin{tabular}{|c|c|c|c|c|c|c|c|c|c|c|c|}
\hline Plant Name & Utility Name & State & Utility/Plc & $n t / U n i$ & $I D$ & $M W$ & Status & Year & Fuel & Water & Cooling System \\
\hline BARRY & $\begin{array}{l}\text { ALABAMA POWER } \\
\text { CO }\end{array}$ & $\mathrm{AL}$ & 195 & 3 & 3 & 272 & Operational & 1959 & COAL & FRESH & ONCE THROUGH \\
\hline BARRY & $\begin{array}{l}\text { ALABAMA POWER } \\
\text { CO }\end{array}$ & $\mathrm{AL}$ & 195 & 3 & 2 & 153 & Operational & 1954 & COAL & FRESH & ONCE THROUGH \\
\hline BARRY & $\begin{array}{l}\text { ALABAMA POWER } \\
\text { CO }\end{array}$ & $\mathrm{AL}$ & 195 & 3 & 1 & 153 & Operational & 1954 & COAL & FRESH & ONCE THROUGH \\
\hline BARTOW & $\begin{array}{l}\text { FLORIDA POWER } \\
\text { CORP }\end{array}$ & $\mathrm{FL}$ & 6455 & 634 & 2 & 128 & Operational & 1961 & OIL & SALINE & ONCE THROUGH \\
\hline BARTOW & $\begin{array}{l}\text { FLORIDA POWER } \\
\text { CORP }\end{array}$ & $\mathrm{FL}$ & 6455 & 634 & 3 & 239 & Operational & 1963 & OIL & SALINE & ONCE THROUGH \\
\hline BARTOW & $\begin{array}{l}\text { FLORIDA POWER } \\
\text { CORP }\end{array}$ & $\mathrm{FL}$ & 6455 & 634 & 1 & 128 & Operational & 1958 & OIL & SALINE & ONCE THROUGH \\
\hline $\begin{array}{l}\text { BAXTER } \\
\text { WILSON }\end{array}$ & $\begin{array}{l}\text { MISSISSIPPI } \\
\text { POWER \& LIGHT }\end{array}$ & MS & 12685 & 2050 & 1 & 545 & Operational & 1966 & GAS & FRESH & ONCE THROUGH \\
\hline $\begin{array}{l}\text { BAXTER } \\
\text { WILSON }\end{array}$ & $\begin{array}{l}\text { MISSISSIPPI } \\
\text { POWER \& LIGHT }\end{array}$ & MS & 12685 & 3050 & 2 & 783 & Operational & 1971 & OIL & FRESH & ONCE THROUGH \\
\hline BAY FRONT & $\begin{array}{l}\text { NORTHERN STATES } \\
\text { POWER CO }\end{array}$ & WI & 13781 & 3982 & 4 & 20 & Operational & 1954 & WOOD & FRESH & ONCE THROUGH \\
\hline BAY FRONT & $\begin{array}{l}\text { NORTHERN STATES } \\
\text { POWER CO }\end{array}$ & WI & 13781 & 3982 & 6 & 27 & Operational & 1960 & WOOD & FRESH & ONCE THROUGH \\
\hline BAY FRONT & $\begin{array}{l}\text { NORTHERN STATES } \\
\text { POWER CO }\end{array}$ & WI & 13781 & 3982 & 5 & 20 & Operational & 1952 & COAL & FRESH & ONCE THROUGH \\
\hline BAY SHORE & TOLEDO EDISON CO & $\mathrm{OH}$ & 18997 & 2878 & 1 & 141 & Operational & 1955 & COAL & FRESH & ONCE THROUGH \\
\hline BAY SHORE & TOLEDO EDISON CO & $\mathrm{OH}$ & 18997 & 2878 & 2 & 141 & Operational & 1959 & COAL & FRESH & ONCE THROUGH \\
\hline BAY SHORE & TOLEDO EDISON CO & $\mathrm{OH}$ & 18997 & 2878 & 3 & 141 & Operational & 1963 & COAL & FRESH & ONCE THROUGH \\
\hline
\end{tabular}




\begin{tabular}{|c|c|c|c|c|c|c|c|c|c|c|c|}
\hline Plant Name & Utility Name & State & Utility/Pl & $n t / U n i$ & $I D$ & $M W$ & Status & Year & Fuel & Water & Cooling System \\
\hline BAY SHORE & TOLEDO EDISON CO & $\mathrm{OH}$ & 18997 & 2878 & 4 & 218 & Operational & 1968 & COAL & FRESH & ONCE THROUGH \\
\hline BAYSIDE & $\begin{array}{l}\text { TRAVERSE CITY LT } \\
\text { \& POWER }\end{array}$ & $\mathrm{Ml}$ & 19125 & 1859 & 2 & 5 & Standby & 1950 & COAL & FRESH & ONCE THROUGH \\
\hline BAYSIDE & $\begin{array}{l}\text { TRAVERSE CITY LT } \\
\text { \& POWER }\end{array}$ & Ml & 19125 & 1859 & 3 & 7.5 & Standby & 1954 & GAS & FRESH & ONCE THROUGH \\
\hline BAYSIDE & $\begin{array}{l}\text { TRAVERSE CITY LT } \\
\text { \& POWER }\end{array}$ & MI & 19125 & 1859 & 1 & 2.5 & Standby & 1946 & COAL & FRESH & ONCE THROUGH \\
\hline BAYSIDE & $\begin{array}{l}\text { TRAVERSE CITY LT } \\
\text { \& POWER }\end{array}$ & $\mathrm{Ml}$ & 19125 & 1859 & 4 & 16.5 & Operational & 1968 & COAL & FRESH & ONCE THROUGH \\
\hline BC COBB & $\begin{array}{l}\text { CONSUMERS } \\
\text { POWER CO }\end{array}$ & Ml & 4254 & 1695 & 4 & 156 & Operational & 1956 & COAL & FRESH & ONCE THROUGH \\
\hline BC COBB & $\begin{array}{l}\text { CONSUMERS } \\
\text { POWER CO }\end{array}$ & Ml & 4254 & 1695 & 5 & 156 & Operational & 1957 & COAL & FRESH & ONCE THROUGH \\
\hline BELLE RIVER & $\begin{array}{l}\text { DETROIT EDISON } \\
\text { CO }\end{array}$ & $\mathrm{Ml}$ & 5109 & 6034 & 2 & 698 & Operational & 1985 & COAL & FRESH & ONCE THROUGH \\
\hline BELLE RIVER & $\begin{array}{l}\text { DETROIT EDISON } \\
\text { CO }\end{array}$ & Ml & 5109 & 6034 & 1 & 698 & Operational & 1984 & COAL & FRESH & ONCE THROUGH \\
\hline BERGEN & $\begin{array}{l}\text { PUBLIC SERVICE } \\
\text { ELEC \& GAS }\end{array}$ & $\mathrm{NJ}$ & 15477 & 2398 & 1 & 325 & Operational & 1959 & GAS & BRACKISH & ONCE THROUGH \\
\hline BERGEN & $\begin{array}{l}\text { PUBLIC SERVICE } \\
\text { ELEC \& GAS }\end{array}$ & $\mathrm{NJ}$ & 15477 & 2398 & 2 & 325 & Operational & 1960 & GAS & BRACKISH & ONCE THROUGH \\
\hline BF CLEARY & $\begin{array}{l}\text { TAUNTON MUNI } \\
\text { LIGHT CO }\end{array}$ & MA & 18488 & 1682 & 8 & 28.3 & Operational & 1966 & OIL & BRACKISH & ONCE THROUGH \\
\hline BIG BEND (FL) & $\begin{array}{l}\text { TAMPA ELECTRIC } \\
\text { CO }\end{array}$ & $\mathrm{FL}$ & 18454 & 645 & 4 & 486 & Operational & 1985 & COAL & SALINE & ONCE THROUGH \\
\hline BIG BEND (FL) & $\begin{array}{l}\text { TAMPA ELECTRIC } \\
\text { CO }\end{array}$ & $\mathrm{FL}$ & 18454 & 645 & 3 & 446 & Operational & 1976 & COAL & SALINE & ONCE THROUGH \\
\hline
\end{tabular}




\begin{tabular}{|c|c|c|c|c|c|c|c|c|c|c|c|}
\hline Plant Name & Utility Name & State & Utility/Pl & tt/Uni & $I D$ & $\boldsymbol{M W}$ & Status & Year & Fuel & Water & Cooling System \\
\hline BIG BEND (FL) & $\begin{array}{l}\text { TAMPA ELECTRIC } \\
\text { CO }\end{array}$ & $\mathrm{FL}$ & 18454 & 645 & 1 & 446 & Operational & 1970 & COAL & SALINE & ONCE THROUGH \\
\hline BIG BEND (FL) & $\begin{array}{l}\text { TAMPA ELECTRIC } \\
\text { CO }\end{array}$ & $\mathrm{FL}$ & 18454 & 645 & 2 & 446 & Operational & 1973 & COAL & SALINE & ONCE THROUGH \\
\hline BIG BROWN & $\begin{array}{l}\text { TEXAS UTILITIES } \\
\text { ELEC CO }\end{array}$ & TX & 44372 & 3497 & 1 & 593 & Operational & 1971 & COAL & FRESH & ONCE THROUGH \\
\hline BIG BROWN & $\begin{array}{l}\text { TEXAS UTILITIES } \\
\text { ELEC CO }\end{array}$ & $\mathrm{TX}$ & 44372 & 3497 & 2 & 593 & Operational & 1972 & COAL & FRESH & ONCE THROUGH \\
\hline $\begin{array}{l}\text { BIG CAJUN } \\
\text { TWO }\end{array}$ & $\begin{array}{l}\text { CAJUN ELECTRIC } \\
\text { POWER COOP }\end{array}$ & LA & 2777 & 6055 & 3 & 560 & Operational & 1983 & COAL & FRESH & ONCE THROUGH \\
\hline BL ENGLAND & $\begin{array}{l}\text { ATLANTIC CITY } \\
\text { ELECTRIC CO }\end{array}$ & NJ & 963 & 2378 & 2 & 163 & Operational & 1964 & COAL & BRACKISH & ONCE THROUGH \\
\hline BL ENGLAND & $\begin{array}{l}\text { ATLANTIC CITY } \\
\text { ELECTRIC CO }\end{array}$ & $\mathrm{NJ}$ & 963 & 2378 & 1 & 136 & Operational & 1962 & COAL & BRACKISH & ONCE THROUGH \\
\hline BLACK DOG & $\begin{array}{l}\text { NORTHERN STATES } \\
\text { POWER CO }\end{array}$ & $\mathrm{MN}$ & 13781 & 1904 & 1 & 81 & Operational & 1952 & COAL & FRESH & $\begin{array}{l}\text { COMBINATION (ONCE } \\
\text { THROUGH/HELPER POND) }\end{array}$ \\
\hline BLACK DOG & $\begin{array}{l}\text { NORTHERN STATES } \\
\text { POWER CO }\end{array}$ & $\mathrm{MN}$ & 13781 & 1904 & 2 & 100 & Operational & 1954 & COAL & FRESH & $\begin{array}{l}\text { COMBINATION (ONCE } \\
\text { THROUGH/HELPER POND) }\end{array}$ \\
\hline BLACK DOG & $\begin{array}{l}\text { NORTHERN STATES } \\
\text { POWER CO }\end{array}$ & MN & 13781 & 1904 & 3 & 102 & Operational & 1955 & COAL & FRESH & $\begin{array}{l}\text { COMBINATION (ONCE } \\
\text { THROUGH/HELPER POND) }\end{array}$ \\
\hline BLACK DOG & $\begin{array}{l}\text { NORTHERN STATES } \\
\text { POWER CO }\end{array}$ & $\mathrm{MN}$ & 13781 & 1904 & 4 & 162 & Operational & 1960 & COAL & FRESH & $\begin{array}{l}\text { COMBINATION (ONCE } \\
\text { THROUGH/HELPER POND) }\end{array}$ \\
\hline BLACKHAWK & $\begin{array}{l}\text { WISCONSIN } \\
\text { POWER \& LIGHT }\end{array}$ & WI & 20856 & 4048 & 3 & 25 & Operational & 1946 & GAS & FRESH & ONCE THROUGH \\
\hline BLACKHAWK & $\begin{array}{l}\text { WISCONSIN } \\
\text { POWER \& LIGHT }\end{array}$ & WI & 20856 & 4048 & 4 & 25 & Operational & 1948 & GAS & FRESH & ONCE THROUGH \\
\hline $\begin{array}{l}\text { BLACKSTONE } \\
\text { STREET }\end{array}$ & $\begin{array}{l}\text { CAMBRIDGE ELEC } \\
\text { LIGHT CO }\end{array}$ & MA & 2886 & 1594 & 1 & 12.5 & Operational & 1930 & GAS & FRESH & ONCE THROUGH \\
\hline
\end{tabular}




\begin{tabular}{|c|c|c|c|c|c|c|c|c|c|c|c|}
\hline Plant Name & Utility Name & State & Utility/Pl & $n t / U n i$ & $I D$ & $M W$ & Status & Year & Fuel & Water & Cooling System \\
\hline $\begin{array}{l}\text { BLOUNT } \\
\text { STREET }\end{array}$ & $\begin{array}{l}\text { MADISON GAS \& } \\
\text { ELEC CO }\end{array}$ & WI & 11479 & 3992 & 6 & 44 & Operational & 1957 & GAS & FRESH & ONCE THROUGH \\
\hline $\begin{array}{l}\text { BLOUNT } \\
\text { STREET }\end{array}$ & $\begin{array}{l}\text { MADISON GAS \& } \\
\text { ELEC CO }\end{array}$ & WI & 11479 & 3992 & 7 & 44 & Operational & 1961 & GAS & FRESH & ONCE THROUGH \\
\hline $\begin{array}{l}\text { BLOUNT } \\
\text { STREET }\end{array}$ & $\begin{array}{l}\text { MADISON GAS \& } \\
\text { ELEC CO }\end{array}$ & WI & 11479 & 3992 & 5 & 25 & Operational & 1948 & GAS & FRESH & ONCE THROUGH \\
\hline $\begin{array}{l}\text { BLOUNT } \\
\text { STREET }\end{array}$ & $\begin{array}{l}\text { MADISON GAS \& } \\
\text { ELEC CO }\end{array}$ & WI & 11479 & 3992 & 4 & 20 & Operational & 1938 & COAL & FRESH & ONCE THROUGH \\
\hline $\begin{array}{l}\text { BLOUNT } \\
\text { STREET }\end{array}$ & $\begin{array}{l}\text { MADISON GAS \& } \\
\text { ELEC CO }\end{array}$ & WI & 11479 & 3992 & 3 & 33 & Operational & 1953 & GAS & FRESH & ONCE THROUGH \\
\hline $\begin{array}{l}\text { BLOUNT } \\
\text { STREET }\end{array}$ & $\begin{array}{l}\text { MADISON GAS \& } \\
\text { ELEC CO }\end{array}$ & WI & 11479 & 3992 & 1 & 12.5 & Operational & 1925 & GAS & FRESH & ONCE THROUGH \\
\hline $\begin{array}{l}\text { BOWLINE } \\
\text { POINT }\end{array}$ & $\begin{array}{l}\text { ORANGE \& } \\
\text { ROCKLAND UTIL }\end{array}$ & NY & 14154 & 2625 & 1 & 621 & Operational & 1972 & OIL & BRACKISH & ONCE THROUGH \\
\hline $\begin{array}{l}\text { BOWLINE } \\
\text { POINT }\end{array}$ & $\begin{array}{l}\text { ORANGE \& } \\
\text { ROCKLAND UTIL }\end{array}$ & NY & 14154 & 2625 & 2 & 621 & Operational & 1974 & GAS & BRACKISH & ONCE THROUGH \\
\hline $\begin{array}{l}\text { BRAYTON } \\
\text { POINT }\end{array}$ & $\begin{array}{l}\text { NEW ENGLAND } \\
\text { POWER CO }\end{array}$ & MA & 13433 & 1619 & 4 & 476 & Operational & 1974 & OIL & SALINE & ONCE THROUGH \\
\hline $\begin{array}{l}\text { BRAYTON } \\
\text { POINT }\end{array}$ & $\begin{array}{l}\text { NEW ENGLAND } \\
\text { POWER CO }\end{array}$ & MA & 13433 & 1619 & 1 & 241 & Operational & 1963 & COAL & SALINE & ONCE THROUGH \\
\hline $\begin{array}{l}\text { BRAYTON } \\
\text { POINT }\end{array}$ & $\begin{array}{l}\text { NEW ENGLAND } \\
\text { POWER CO }\end{array}$ & MA & 13433 & 1619 & 2 & 241 & Operational & 1964 & COAL & SALINE & ONCE THROUGH \\
\hline $\begin{array}{l}\text { BRAYTON } \\
\text { POINT }\end{array}$ & $\begin{array}{l}\text { NEW ENGLAND } \\
\text { POWER CO }\end{array}$ & MA & 13433 & 1619 & 3 & 643 & Operational & 1969 & COAL & SALINE & ONCE THROUGH \\
\hline BREMO BLUFF & $\begin{array}{l}\text { VIRGINIA ELEC \& } \\
\text { POWER CO }\end{array}$ & VA & 19876 & 3796 & 4 & 185 & Operational & 1958 & COAL & FRESH & ONCE THROUGH \\
\hline BREMO BLUFF & $\begin{array}{l}\text { VIRGINIA ELEC \& } \\
\text { POWER CO }\end{array}$ & VA & 19876 & 3796 & 3 & 69 & Operational & 1950 & COAL & FRESH & ONCE THROUGH \\
\hline
\end{tabular}




\begin{tabular}{|c|c|c|c|c|c|c|c|c|c|c|c|}
\hline Plant Name & Utility Name & State & Utility/Plc & $n t / U n i t$ & $I D$ & $M W$ & Status & Year & Fuel & Water & Cooling System \\
\hline $\begin{array}{l}\text { BRIDGEPORT } \\
\text { HARBOR }\end{array}$ & $\begin{array}{l}\text { UNITED } \\
\text { ILLUMINATING CO }\end{array}$ & $\mathrm{CT}$ & 19497 & 568 & 2 & 180 & Operational & 1961 & OIL & SALINE & ONCE THROUGH \\
\hline $\begin{array}{l}\text { BRIDGEPORT } \\
\text { HARBOR }\end{array}$ & $\begin{array}{l}\text { UNITED } \\
\text { ILLUMINATING CO }\end{array}$ & $\mathrm{CT}$ & 19497 & 568 & 3 & 400 & Operational & 1968 & COAL & SALINE & ONCE THROUGH \\
\hline $\begin{array}{l}\text { BROWNS } \\
\text { FERRY }\end{array}$ & $\begin{array}{l}\text { TENNESSEE } \\
\text { VALLEY AUTH }\end{array}$ & $A L$ & 18642 & 46 & 1 & 1152 & Standby & 1974 & UR & FRESH & $\begin{array}{l}\text { MIXED MODE (ONCE } \\
\text { THROUGH/COOLING TOWER) }\end{array}$ \\
\hline $\begin{array}{l}\text { BROWNS } \\
\text { FERRY }\end{array}$ & $\begin{array}{l}\text { TENNESSEE } \\
\text { VALLEY AUTH }\end{array}$ & $\mathrm{AL}$ & 18642 & 46 & 2 & 1152 & Operational & 1975 & UR & FRESH & $\begin{array}{l}\text { MIXED MODE (ONCE } \\
\text { THROUGH/COOLING TOWER) }\end{array}$ \\
\hline $\begin{array}{l}\text { BROWNS } \\
\text { FERRY }\end{array}$ & $\begin{array}{l}\text { TENNESSEE } \\
\text { VALLEY AUTH }\end{array}$ & $A L$ & 18642 & 46 & 3 & 1152 & Operational & 1977 & UR & FRESH & $\begin{array}{l}\text { MIXED MODE (ONCE } \\
\text { THROUGH/COOLING TOWER) }\end{array}$ \\
\hline $\begin{array}{l}\text { BRUNNER } \\
\text { ISLAND }\end{array}$ & $\begin{array}{l}\text { PENNSYLVANIA } \\
\text { POWER \& LT }\end{array}$ & PA & 14715 & 3140 & 1 & 363 & Operational & 1961 & COAL & FRESH & ONCE THROUGH \\
\hline $\begin{array}{l}\text { BRUNNER } \\
\text { ISLAND }\end{array}$ & $\begin{array}{l}\text { PENNSYLVANIA } \\
\text { POWER \& LT }\end{array}$ & PA & 14715 & 3140 & 2 & 405 & Operational & 1965 & COAL & FRESH & ONCE THROUGH \\
\hline $\begin{array}{l}\text { BRUNNER } \\
\text { ISLAND }\end{array}$ & $\begin{array}{l}\text { PENNSYLVANIA } \\
\text { POWER \& LT }\end{array}$ & PA & 14715 & 3140 & 3 & 790 & Operational & 1969 & COAL & FRESH & ONCE THROUGH \\
\hline $\begin{array}{l}\text { BRUNSWICK } \\
\text { (NC) }\end{array}$ & $\begin{array}{l}\text { CAROLINA POWER } \\
\& \text { LIGHT CO }\end{array}$ & NC & 3046 & 6014 & 1 & 867 & Operational & 1977 & UR & SALINE & ONCE THROUGH \\
\hline $\begin{array}{l}\text { BRUNSWICK } \\
\text { (NC) }\end{array}$ & $\begin{array}{l}\text { CAROLINA POWER } \\
\& \text { LIGHT CO }\end{array}$ & NC & 3046 & 6014 & 2 & 867 & Operational & 1975 & UR & SALINE & ONCE THROUGH \\
\hline BUCK (NC) & DUKE POWER CO & NC & 5416 & 2720 & 3 & 80 & Standby & 1941 & COAL & FRESH & ONCE THROUGH \\
\hline BUCK (NC) & DUKE POWER CO & NC & 5416 & 2720 & 4 & 40 & Standby & 1942 & COAL & FRESH & ONCE THROUGH \\
\hline BUCK (NC) & DUKE POWER CO & NC & 5416 & 2720 & 5 & 125 & Operational & 1953 & COAL & FRESH & ONCE THROUGH \\
\hline BUCK (NC) & DUKE POWER CO & NC & 5416 & 2720 & 6 & 125 & Operational & 1953 & COAL & FRESH & ONCE THROUGH \\
\hline
\end{tabular}




\begin{tabular}{|c|c|c|c|c|c|c|c|c|c|c|c|}
\hline Plant Name & Utility Name & State & Utility/P & $n t / U n i$ & $I D$ & $M W$ & Status & Year & Fuel & Water & Cooling System \\
\hline BULL RUN (TN) & $\begin{array}{l}\text { TENNESSEE } \\
\text { VALLEY AUTH }\end{array}$ & $\mathrm{TN}$ & 18642 & 3396 & 1 & 950 & Operational & 1967 & COAL & FRESH & ONCE THROUGH \\
\hline BULLOCK & $\begin{array}{l}\text { TRI-STATE G\&T } \\
\text { ASSN }\end{array}$ & $\mathrm{CO}$ & 30151 & & 1 & 6 & Standby & 1951 & COAL & FRESH & ONCE THROUGH \\
\hline BULLOCK & $\begin{array}{l}\text { TRI-STATE G\&T } \\
\text { ASSN }\end{array}$ & $\mathrm{CO}$ & 30151 & & 2 & 6 & Standby & 1955 & COAL & FRESH & ONCE THROUGH \\
\hline BURDICK & $\begin{array}{l}\text { GRAND ISLAND } \\
\text { WTR \& LIGHT }\end{array}$ & NE & 40606 & 2241 & 1 & 18.8 & Standby & 1957 & GAS & GROUND & ONCE THROUGH \\
\hline BURDICK & $\begin{array}{l}\text { GRAND ISLAND } \\
\text { WTR \& LIGHT }\end{array}$ & NE & 40606 & 2241 & 2 & 25 & Standby & 1963 & GAS & GROUND & ONCE THROUGH \\
\hline $\begin{array}{l}\text { BURLINGTON } \\
\text { (IA) }\end{array}$ & IES UTILITIES INC & IA & 9162 & 1104 & 1 & 212 & Operational & 1968 & COAL & FRESH & ONCE THROUGH \\
\hline $\begin{array}{l}\text { BURLINGTON } \\
\text { (NJ) }\end{array}$ & $\begin{array}{l}\text { PUBLIC SERVICE } \\
\text { ELEC \& GAS }\end{array}$ & NJ & 15477 & 2399 & 7 & 205 & Operational & 1955 & OIL & FRESH & ONCE THROUGH \\
\hline $\begin{array}{l}\text { CABOT- } \\
\text { HOLYOKE }\end{array}$ & $\begin{array}{l}\text { HOLYOKE GAS \& } \\
\text { ELEC DEPT }\end{array}$ & MA & 8776 & 9864 & 8 & 9.4 & Operational & 1951 & OIL & FRESH & ONCE THROUGH \\
\hline $\begin{array}{l}\text { CABOT- } \\
\text { HOLYOKE }\end{array}$ & $\begin{array}{l}\text { HOLYOKE GAS \& } \\
\text { ELEC DEPT }\end{array}$ & MA & 8776 & 9864 & 6 & 9.4 & Operational & 1955 & OIL & FRESH & ONCE THROUGH \\
\hline CABRAS & $\begin{array}{l}\text { GUAM POWER } \\
\text { AUTHORITY }\end{array}$ & GU & 40428 & & 1 & 66 & Operational & 1974 & OIL & SALINE & ONCE THROUGH \\
\hline CABRAS & $\begin{array}{l}\text { GUAM POWER } \\
\text { AUTHORITY }\end{array}$ & GU & 40428 & & 2 & 66 & Operational & 1975 & OIL & SALINE & ONCE THROUGH \\
\hline $\begin{array}{l}\text { CALVERT } \\
\text { CLIFFS }\end{array}$ & $\begin{array}{l}\text { BALTIMORE GAS \& } \\
\text { ELEC CO }\end{array}$ & MD & 1167 & 6011 & 1 & 918 & Operational & 1975 & UR & BRACKISH & ONCE THROUGH \\
\hline $\begin{array}{l}\text { CALVERT } \\
\text { CLIFFS }\end{array}$ & $\begin{array}{l}\text { BALTIMORE GAS \& } \\
\text { ELEC CO }\end{array}$ & MD & 1167 & 6011 & 2 & 918 & Operational & 1977 & UR & BRACKISH & ONCE THROUGH \\
\hline CAMEO & $\begin{array}{l}\text { PUBLIC SERVICE } \\
\text { COLORADO }\end{array}$ & $\mathrm{CO}$ & 15466 & 468 & 1 & 22 & Operational & 1957 & COAL & FRESH & ONCE THROUGH \\
\hline
\end{tabular}




\begin{tabular}{|c|c|c|c|c|c|c|c|c|c|c|c|}
\hline Plant Name & Utility Name & State & Utility/Pl & $n t / U n i t$ & $I D$ & $M W$ & Status & Year & Fuel & Water & Cooling System \\
\hline CAMEO & $\begin{array}{l}\text { PUBLIC SERVICE } \\
\text { COLORADO }\end{array}$ & $\mathrm{CO}$ & 15466 & 468 & 2 & 44 & Operational & 1960 & COAL & FRESH & $\begin{array}{l}\text { COMBINATION (ONCE } \\
\text { THROUGH/SPRAY POND) }\end{array}$ \\
\hline CANADAY & $\begin{array}{l}\text { CEN NEBRASKA } \\
\text { PUB PWR\&IRR }\end{array}$ & NE & 3271 & 2226 & 1 & 109 & Operational & 1958 & GAS & FRESH & ONCE THROUGH \\
\hline CANADYS & $\begin{array}{l}\text { SOUTH CAROLINA } \\
\text { ELEC \& GAS }\end{array}$ & SC & 17539 & 3280 & 1 & 136 & Operational & 1962 & COAL & FRESH & ONCE THROUGH \\
\hline CANADYS & $\begin{array}{l}\text { SOUTH CAROLINA } \\
\text { ELEC \& GAS }\end{array}$ & SC & 17539 & 3280 & 2 & 136 & Operational & 1964 & COAL & FRESH & ONCE THROUGH \\
\hline CANAL & $\begin{array}{l}\text { CANAL ELECTRIC } \\
\text { CO }\end{array}$ & MA & 2951 & 1599 & 1 & 543 & Operational & 1968 & OIL & SALINE & ONCE THROUGH \\
\hline CANAL & $\begin{array}{l}\text { CANAL ELECTRIC } \\
\text { CO }\end{array}$ & MA & 2951 & 1599 & 2 & 530 & Operational & 1976 & OIL & SALINE & ONCE THROUGH \\
\hline CANE RUN & $\begin{array}{l}\text { LOUISVILLE GAS \& } \\
\text { ELEC CO }\end{array}$ & KY & 11249 & 1363 & 6 & 272 & Operational & 1969 & COAL & FRESH & ONCE THROUGH \\
\hline CANE RUN & $\begin{array}{l}\text { LOUISVILLE GAS \& } \\
\text { ELEC CO }\end{array}$ & KY & 11249 & 1363 & 3 & 147 & Standby & 1958 & GAS & FRESH & ONCE THROUGH \\
\hline CANE RUN & $\begin{array}{l}\text { LOUISVILLE GAS \& } \\
\text { ELEC CO }\end{array}$ & KY & 11249 & 1363 & 4 & 163 & Operational & 1962 & COAL & FRESH & ONCE THROUGH \\
\hline CANE RUN & $\begin{array}{l}\text { LOUISVILLE GAS \& } \\
\text { ELEC CO }\end{array}$ & KY & 11249 & 1363 & 5 & 209 & Operational & 1966 & COAL & FRESH & ONCE THROUGH \\
\hline $\begin{array}{l}\text { CAPE } \\
\text { CANAVERAL }\end{array}$ & $\begin{array}{l}\text { FLORIDA POWER \& } \\
\text { LIGHT CO }\end{array}$ & $\mathrm{FL}$ & 6452 & 609 & 2 & 402 & Operational & 1969 & OIL & BRACKISH & ONCE THROUGH \\
\hline $\begin{array}{l}\text { CAPE } \\
\text { CANAVERAL }\end{array}$ & $\begin{array}{l}\text { FLORIDA POWER \& } \\
\text { LIGHT CO }\end{array}$ & $\mathrm{FL}$ & 6452 & 609 & 1 & 402 & Operational & 1965 & OIL & BRACKISH & ONCE THROUGH \\
\hline CAPE FEAR & $\begin{array}{l}\text { CAROLINA POWER } \\
\& \text { LIGHT CO }\end{array}$ & NC & 3046 & 2708 & 5 & 141 & Operational & 1956 & COAL & FRESH & $\begin{array}{l}\text { COMBINATION (ONCE } \\
\text { THROUGH/HELPER TOWER) }\end{array}$ \\
\hline CAPE FEAR & $\begin{array}{l}\text { CAROLINA POWER } \\
\& \text { LIGHT CO }\end{array}$ & NC & 3046 & 2708 & 6 & 188 & Operational & 1958 & COAL & FRESH & $\begin{array}{l}\text { COMBINATION (ONCE } \\
\text { THROUGH/HELPER TOWER) }\end{array}$ \\
\hline
\end{tabular}




\begin{tabular}{|c|c|c|c|c|c|c|c|c|c|c|c|}
\hline Plant Name & Utility Name & State & Utility/Pl & nt/Uni & $I D$ & $M W$ & Status & Year & Fuel & Water & Cooling System \\
\hline CARDINAL & $\begin{array}{l}\text { CARDINAL } \\
\text { OPERATING CO }\end{array}$ & $\mathrm{OH}$ & 3006 & 2828 & 1 & 615 & Operational & 1967 & COAL & FRESH & ONCE THROUGH \\
\hline CARDINAL & $\begin{array}{l}\text { CARDINAL } \\
\text { OPERATING CO }\end{array}$ & $\mathrm{OH}$ & 3006 & 2828 & 2 & 615 & Operational & 1967 & COAL & FRESH & ONCE THROUGH \\
\hline CARLSON & $\begin{array}{l}\text { JAMESTOWN BD OF } \\
\text { PUB UTIL }\end{array}$ & NY & 9645 & 2682 & 5 & 30.5 & Operational & 1951 & COAL & FRESH & ONCE THROUGH \\
\hline CARLSON & $\begin{array}{l}\text { JAMESTOWN BD OF } \\
\text { PUB UTIL }\end{array}$ & NY & 9645 & 2682 & 6 & 27.2 & Operational & 1968 & COAL & FRESH & ONCE THROUGH \\
\hline CAYUGA & PSI ENERGY INC & IN & 15470 & 1001 & 1 & 531 & Operational & 1970 & COAL & FRESH & $\begin{array}{l}\text { COMBINATION (ONCE } \\
\text { THROUGH/HELPER TOWER) }\end{array}$ \\
\hline CAYUGA & PSI ENERGY INC & IN & 15470 & 1001 & 2 & 531 & Operational & 1972 & COAL & FRESH & $\begin{array}{l}\text { COMBINATION (ONCE } \\
\text { THROUGH/HELPER TOWER) }\end{array}$ \\
\hline CEDAR BAYOU & $\begin{array}{l}\text { HOUSTON } \\
\text { LIGHTING \& POWER }\end{array}$ & TX & 8901 & 3460 & 1 & 765 & Operational & 1970 & GAS & BRACKISH & $\begin{array}{l}\text { COMBINATION (ONCE } \\
\text { THROUGH/HELPER POND) }\end{array}$ \\
\hline CEDAR BAYOU & $\begin{array}{l}\text { HOUSTON } \\
\text { LIGHTING \& POWER }\end{array}$ & $\mathrm{TX}$ & 8901 & 3460 & 2 & 765 & Operational & 1972 & GAS & BRACKISH & $\begin{array}{l}\text { COMBINATION (ONCE } \\
\text { THROUGH/HELPER POND) }\end{array}$ \\
\hline CEDAR BAYOU & $\begin{array}{l}\text { HOUSTON } \\
\text { LIGHTING \& POWER }\end{array}$ & $\mathrm{TX}$ & 8901 & 3460 & 3 & 765 & Operational & 1974 & GAS & BRACKISH & $\begin{array}{l}\text { COMBINATION (ONCE } \\
\text { THROUGH/HELPER POND) }\end{array}$ \\
\hline CHALK POINT & $\begin{array}{l}\text { POTOMAC } \\
\text { ELECTRIC POWER }\end{array}$ & MD & 15270 & 1571 & 1 & 364 & Operational & 1964 & COAL & BRACKISH & ONCE THROUGH \\
\hline CHALK POINT & $\begin{array}{l}\text { POTOMAC } \\
\text { ELECTRIC POWER }\end{array}$ & MD & 15270 & 1571 & 2 & 364 & Operational & 1965 & COAL & BRACKISH & ONCE THROUGH \\
\hline CHAMOIS & $\begin{array}{l}\text { CENT ELEC PWR } \\
\operatorname{COOP}(\mathrm{MO})\end{array}$ & MO & 3242 & 2169 & 1 & 15 & Operational & 1953 & COAL & FRESH & ONCE THROUGH \\
\hline CHAMOIS & $\begin{array}{l}\text { CENT ELEC PWR } \\
\text { COOP (MO) }\end{array}$ & MO & 3242 & 2169 & 2 & 44 & Operational & 1960 & COAL & FRESH & ONCE THROUGH \\
\hline $\begin{array}{l}\text { CHARLES } \\
\text { POLETTI }\end{array}$ & $\begin{array}{l}\text { NEW YORK POWER } \\
\text { AUTHORITY }\end{array}$ & NY & 15296 & 2491 & 1 & 883 & Operational & 1977 & GAS & SALINE & ONCE THROUGH \\
\hline
\end{tabular}




\begin{tabular}{|c|c|c|c|c|c|c|c|c|c|c|c|}
\hline Plant Name & Utility Name & State & Utility/Pl & $n t / U n i$ & $I D$ & $M W$ & Status & Year & Fuel & Water & Cooling System \\
\hline CHENA & $\begin{array}{l}\text { FAIRBANKS } \\
\text { UTILITIES SYS }\end{array}$ & AK & 6129 & 79 & 1 & 5 & Operational & 1951 & COAL & GROUND & ONCE THROUGH \\
\hline CHENA & $\begin{array}{l}\text { FAIRBANKS } \\
\text { UTILITIES SYS }\end{array}$ & AK & 6129 & 79 & 2 & 2 & Operational & 1951 & COAL & GROUND & ONCE THROUGH \\
\hline CHENA & $\begin{array}{l}\text { FAIRBANKS } \\
\text { UTILITIES SYS }\end{array}$ & AK & 6129 & 79 & 3 & 1.5 & Operational & 1951 & COAL & GROUND & ONCE THROUGH \\
\hline CHENA & $\begin{array}{l}\text { FAIRBANKS } \\
\text { UTILITIES SYS }\end{array}$ & AK & 6129 & 79 & 5 & 20 & Operational & 1970 & COAL & GROUND & ONCE THROUGH \\
\hline CHESAPEAKE & $\begin{array}{l}\text { VIRGINIA ELEC \& } \\
\text { POWER CO }\end{array}$ & VA & 19876 & 3803 & 2 & 113 & Operational & 1954 & COAL & BRACKISH & ONCE THROUGH \\
\hline CHESAPEAKE & $\begin{array}{l}\text { VIRGINIA ELEC \& } \\
\text { POWER CO }\end{array}$ & VA & 19876 & 3803 & 3 & 185 & Operational & 1959 & COAL & BRACKISH & ONCE THROUGH \\
\hline CHESAPEAKE & $\begin{array}{l}\text { VIRGINIA ELEC \& } \\
\text { POWER CO }\end{array}$ & VA & 19876 & 3803 & 4 & 239 & Operational & 1962 & COAL & BRACKISH & ONCE THROUGH \\
\hline CHESAPEAKE & $\begin{array}{l}\text { VIRGINIA ELEC \& } \\
\text { POWER CO }\end{array}$ & VA & 19876 & 3803 & 1 & 113 & Operational & 1953 & COAL & BRACKISH & ONCE THROUGH \\
\hline $\begin{array}{l}\text { CHESTERFIEL } \\
\text { D }\end{array}$ & $\begin{array}{l}\text { VIRGINIA ELEC \& } \\
\text { POWER CO }\end{array}$ & VA & 19876 & 3797 & 6 & 694 & Operational & 1969 & COAL & FRESH & ONCE THROUGH \\
\hline $\begin{array}{l}\text { CHESTERFIEL } \\
\text { D }\end{array}$ & $\begin{array}{l}\text { VIRGINIA ELEC \& } \\
\text { POWER CO }\end{array}$ & VA & 19876 & 3797 & 3 & 113 & Operational & 1952 & COAL & FRESH & ONCE THROUGH \\
\hline $\begin{array}{l}\text { CHESTERFIEL } \\
\text { D }\end{array}$ & $\begin{array}{l}\text { VIRGINIA ELEC \& } \\
\text { POWER CO }\end{array}$ & VA & 19876 & 3797 & 4 & 188 & Operational & 1960 & COAL & FRESH & ONCE THROUGH \\
\hline $\begin{array}{l}\text { CHESTERFIEL } \\
\text { D }\end{array}$ & $\begin{array}{l}\text { VIRGINIA ELEC \& } \\
\text { POWER CO }\end{array}$ & VA & 19876 & 3797 & 5 & 359 & Operational & 1964 & COAL & FRESH & ONCE THROUGH \\
\hline CHESWICK & $\begin{array}{l}\text { DUQUESNE LIGHT } \\
\text { CO }\end{array}$ & PA & 5487 & 8226 & 1 & 565 & Operational & 1970 & COAL & FRESH & ONCE THROUGH \\
\hline CHICKASAW & $\begin{array}{l}\text { ALABAMA POWER } \\
\text { CO }\end{array}$ & $\mathrm{AL}$ & 195 & 5 & 3 & 46 & Operational & 1951 & GAS & FRESH & ONCE THROUGH \\
\hline
\end{tabular}




\begin{tabular}{|c|c|c|c|c|c|c|c|c|c|c|c|}
\hline Plant Name & Utility Name & State & Utility/Pl & $n t / U n$ & $I D$ & $M W$ & Status & Year & Fuel & Water & Cooling System \\
\hline $\begin{array}{l}\text { CLAY } \\
\text { BOSWELL }\end{array}$ & $\begin{array}{l}\text { MINNESOTA } \\
\text { POWER \& LIGHT }\end{array}$ & $\mathrm{MN}$ & 12647 & 1893 & 1 & 75 & Operational & 1958 & COAL & FRESH & ONCE THROUGH \\
\hline $\begin{array}{l}\text { CLAY } \\
\text { BOSWELL }\end{array}$ & $\begin{array}{l}\text { MINNESOTA } \\
\text { POWER \& LIGHT }\end{array}$ & $\mathrm{MN}$ & 12647 & 1893 & 2 & 75 & Operational & 1960 & COAL & FRESH & ONCE THROUGH \\
\hline CLIFFSIDE & DUKE POWER CO & NC & 5416 & 2721 & 1 & 40 & Standby & 1940 & COAL & FRESH & ONCE THROUGH \\
\hline CLIFFSIDE & DUKE POWER CO & NC & 5416 & 2721 & 2 & 40 & Standby & 1940 & COAL & FRESH & ONCE THROUGH \\
\hline CLIFFSIDE & DUKE POWER CO & NC & 5416 & 2721 & 3 & 65 & Standby & 1948 & COAL & FRESH & ONCE THROUGH \\
\hline CLIFFSIDE & DUKE POWER CO & NC & 5416 & 2721 & 4 & 65 & Standby & 1948 & COAL & FRESH & ONCE THROUGH \\
\hline CLIFTY CREEK & $\begin{array}{l}\text { INDIANA KENTUCKY } \\
\text { ELEC COR }\end{array}$ & IN & 9269 & 983 & 2 & 217 & Operational & 1955 & COAL & FRESH & ONCE THROUGH \\
\hline CLIFTY CREEK & $\begin{array}{l}\text { INDIANA KENTUCKY } \\
\text { ELEC COR }\end{array}$ & IN & 9269 & 983 & 6 & 217 & Operational & 1956 & COAL & FRESH & ONCE THROUGH \\
\hline CLIFTY CREEK & $\begin{array}{l}\text { INDIANA KENTUCKY } \\
\text { ELEC COR }\end{array}$ & IN & 9269 & 983 & 5 & 217 & Operational & 1955 & COAL & FRESH & ONCE THROUGH \\
\hline CLIFTY CREEK & $\begin{array}{l}\text { INDIANA KENTUCKY } \\
\text { ELEC COR }\end{array}$ & IN & 9269 & 983 & 3 & 217 & Operational & 1955 & COAL & FRESH & ONCE THROUGH \\
\hline CLIFTY CREEK & $\begin{array}{l}\text { INDIANA KENTUCKY } \\
\text { ELEC COR }\end{array}$ & IN & 9269 & 983 & 1 & 217 & Operational & 1955 & COAL & FRESH & ONCE THROUGH \\
\hline CLIFTY CREEK & $\begin{array}{l}\text { INDIANA KENTUCKY } \\
\text { ELEC COR }\end{array}$ & IN & 9269 & 983 & 4 & 217 & Operational & 1955 & COAL & FRESH & ONCE THROUGH \\
\hline COLBERT & $\begin{array}{l}\text { TENNESSEE } \\
\text { VALLEY AUTH }\end{array}$ & $\mathrm{AL}$ & 18642 & 47 & 1 & 200 & Operational & 1955 & COAL & FRESH & ONCE THROUGH \\
\hline COLBERT & $\begin{array}{l}\text { TENNESSEE } \\
\text { VALLEY AUTH }\end{array}$ & $\mathrm{AL}$ & 18642 & 47 & 5 & 550 & Operational & 1965 & COAL & FRESH & ONCE THROUGH \\
\hline
\end{tabular}




\begin{tabular}{|c|c|c|c|c|c|c|c|c|c|c|c|}
\hline Plant Name & Utility Name & State & Utility/Pl & $n t / U n i$ & $I D$ & $M W$ & Status & Year & Fuel & Water & Cooling System \\
\hline COLBERT & $\begin{array}{l}\text { TENNESSEE } \\
\text { VALLEY AUTH }\end{array}$ & $\mathrm{AL}$ & 18642 & 47 & 4 & 200 & Operational & 1955 & COAL & FRESH & ONCE THROUGH \\
\hline COLBERT & $\begin{array}{l}\text { TENNESSEE } \\
\text { VALLEY AUTH }\end{array}$ & $\mathrm{AL}$ & 18642 & 47 & 2 & 200 & Operational & 1955 & COAL & FRESH & ONCE THROUGH \\
\hline COLBERT & $\begin{array}{l}\text { TENNESSEE } \\
\text { VALLEY AUTH }\end{array}$ & $\mathrm{AL}$ & 18642 & 47 & 3 & 200 & Operational & 1955 & COAL & FRESH & ONCE THROUGH \\
\hline COLEMAN (KY) & $\begin{array}{l}\text { BIG RIVERS ELEC } \\
\text { CORP }\end{array}$ & KY & 1692 & 1381 & 3 & 173 & Operational & 1972 & COAL & FRESH & ONCE THROUGH \\
\hline COLEMAN (KY) & $\begin{array}{l}\text { BIG RIVERS ELEC } \\
\text { CORP }\end{array}$ & KY & 1692 & 1381 & 1 & 174 & Operational & 1969 & COAL & FRESH & ONCE THROUGH \\
\hline COLEMAN (KY) & $\begin{array}{l}\text { BIG RIVERS ELEC } \\
\text { CORP }\end{array}$ & KY & 1692 & 1381 & 2 & 174 & Operational & 1970 & COAL & FRESH & ONCE THROUGH \\
\hline CONESVILLE & $\begin{array}{l}\text { COLUMBUS } \\
\text { SOUTHERN POWER }\end{array}$ & $\mathrm{OH}$ & 4062 & 2840 & 3 & 162 & Operational & 1962 & COAL & FRESH & ONCE THROUGH \\
\hline CONESVILLE & $\begin{array}{l}\text { COLUMBUS } \\
\text { SOUTHERN POWER }\end{array}$ & $\mathrm{OH}$ & 4062 & 2840 & 1 & 148 & Operational & 1959 & COAL & FRESH & ONCE THROUGH \\
\hline CONESVILLE & $\begin{array}{l}\text { COLUMBUS } \\
\text { SOUTHERN POWER }\end{array}$ & $\mathrm{OH}$ & 4062 & 2840 & 2 & 136 & Operational & 1957 & COAL & FRESH & ONCE THROUGH \\
\hline $\begin{array}{l}\text { CONNORS } \\
\text { CREEK }\end{array}$ & $\begin{array}{l}\text { DETROIT EDISON } \\
\text { CO }\end{array}$ & MI & 5109 & 1726 & 16 & 150 & Standby & 1951 & COAL & FRESH & ONCE THROUGH \\
\hline $\begin{array}{l}\text { CONNORS } \\
\text { CREEK }\end{array}$ & $\begin{array}{l}\text { DETROIT EDISON } \\
\text { CO }\end{array}$ & MI & 5109 & 1726 & 15 & 150 & Standby & 1951 & COAL & FRESH & ONCE THROUGH \\
\hline $\begin{array}{l}\text { CONTRA } \\
\text { COSTA }\end{array}$ & $\begin{array}{l}\text { PACIFIC GAS \& } \\
\text { ELEC CO }\end{array}$ & $\mathrm{CA}$ & 14328 & 228 & 6 & 359 & Operational & 1964 & GAS & BRACKISH & ONCE THROUGH \\
\hline $\begin{array}{l}\text { CONTRA } \\
\text { COSTA }\end{array}$ & $\begin{array}{l}\text { PACIFIC GAS \& } \\
\text { ELEC CO }\end{array}$ & $\mathrm{CA}$ & 14328 & 228 & 7 & 359 & Operational & 1964 & GAS & BRACKISH & ONCE THROUGH \\
\hline COOPER & $\begin{array}{l}\text { NEBRASKA PUBLIC } \\
\text { POWER DIS }\end{array}$ & NE & 13337 & 8036 & 1 & 836 & Operational & 1974 & UR & FRESH & ONCE THROUGH \\
\hline
\end{tabular}




\begin{tabular}{|c|c|c|c|c|c|c|c|c|c|c|c|}
\hline Plant Name & Utility Name & State & Utility/Plc & $n t / U n i t$ & $I D$ & $M W$ & Status & Year & Fuel & Water & Cooling System \\
\hline COSTA SUR & $\begin{array}{l}\text { PUERTO RICO ELEC } \\
\text { PWR AUTH }\end{array}$ & PR & 15497 & & 1 & 44 & Operational & 1957 & OIL & SALINE & ONCE THROUGH \\
\hline COSTA SUR & $\begin{array}{l}\text { PUERTO RICO ELEC } \\
\text { PWR AUTH }\end{array}$ & PR & 15497 & & 6 & 462 & Operational & 1973 & OIL & SALINE & ONCE THROUGH \\
\hline COSTA SUR & $\begin{array}{l}\text { PUERTO RICO ELEC } \\
\text { PWR AUTH }\end{array}$ & PR & 15497 & & 5 & 462 & Operational & 1972 & OIL & SALINE & ONCE THROUGH \\
\hline COSTA SUR & $\begin{array}{l}\text { PUERTO RICO ELEC } \\
\text { PWR AUTH }\end{array}$ & PR & 15497 & & 4 & 93.8 & Operational & 1968 & OIL & SALINE & ONCE THROUGH \\
\hline COSTA SUR & $\begin{array}{l}\text { PUERTO RICO ELEC } \\
\text { PWR AUTH }\end{array}$ & PR & 15497 & & 2 & 44 & Operational & 1958 & OIL & SALINE & ONCE THROUGH \\
\hline COSTA SUR & $\begin{array}{l}\text { PUERTO RICO ELEC } \\
\text { PWR AUTH }\end{array}$ & PR & 15497 & & 3 & 93.8 & Operational & 1967 & OIL & SALINE & ONCE THROUGH \\
\hline $\begin{array}{l}\text { COUNCIL } \\
\text { BLUFFS }\end{array}$ & IOWA POWER INC & $\mathrm{IA}$ & 9429 & 1082 & 3 & 726 & Operational & 1978 & COAL & FRESH & ONCE THROUGH \\
\hline $\begin{array}{l}\text { COUNCIL } \\
\text { BLUFFS }\end{array}$ & IOWA POWER INC & $\mathrm{IA}$ & 9429 & 1082 & 2 & 81.6 & Operational & 1958 & COAL & FRESH & ONCE THROUGH \\
\hline $\begin{array}{l}\text { COUNCIL } \\
\text { BLUFFS }\end{array}$ & IOWA POWER INC & IA & 9429 & 1082 & 1 & 49 & Operational & 1954 & COAL & FRESH & ONCE THROUGH \\
\hline CP CRANE & $\begin{array}{l}\text { BALTIMORE GAS \& } \\
\text { ELEC CO }\end{array}$ & $\mathrm{MD}$ & 1167 & 1552 & 1 & 190 & Operational & 1961 & COAL & BRACKISH & ONCE THROUGH \\
\hline CP CRANE & $\begin{array}{l}\text { BALTIMORE GAS \& } \\
\text { ELEC CO }\end{array}$ & MD & 1167 & 1552 & 2 & 209 & Operational & 1963 & COAL & BRACKISH & ONCE THROUGH \\
\hline CR HUNTLEY & $\begin{array}{l}\text { NIAGARA MOHAWK } \\
\text { POWER CORP }\end{array}$ & NY & 13573 & 2549 & 66 & 100 & Operational & 1954 & COAL & FRESH & ONCE THROUGH \\
\hline CR HUNTLEY & $\begin{array}{l}\text { NIAGARA MOHAWK } \\
\text { POWER CORP }\end{array}$ & NY & 13573 & 2549 & 67 & 218 & Operational & 1957 & COAL & FRESH & ONCE THROUGH \\
\hline CR HUNTLEY & $\begin{array}{l}\text { NIAGARA MOHAWK } \\
\text { POWER CORP }\end{array}$ & NY & 13573 & 2549 & 65 & 100 & Operational & 1953 & COAL & FRESH & ONCE THROUGH \\
\hline
\end{tabular}




\begin{tabular}{|c|c|c|c|c|c|c|c|c|c|c|c|}
\hline Plant Name & Utility Name & State & Utility/Pla & $n t / U n i$ & $I D$ & $M W$ & Status & Year & Fuel & Water & Cooling System \\
\hline CR HUNTLEY & $\begin{array}{l}\text { NIAGARA MOHAWK } \\
\text { POWER CORP }\end{array}$ & NY & 13573 & 2549 & 64 & 100 & Operational & 1948 & COAL & FRESH & ONCE THROUGH \\
\hline CR HUNTLEY & $\begin{array}{l}\text { NIAGARA MOHAWK } \\
\text { POWER CORP }\end{array}$ & NY & 13573 & 2549 & 63 & 92 & Operational & 1942 & COAL & FRESH & ONCE THROUGH \\
\hline CR HUNTLEY & $\begin{array}{l}\text { NIAGARA MOHAWK } \\
\text { POWER CORP }\end{array}$ & NY & 13573 & 2549 & 68 & 218 & Operational & 1958 & COAL & FRESH & ONCE THROUGH \\
\hline CR LOWMAN & $\begin{array}{l}\text { ALABAMA ELECTRIC } \\
\text { COOP }\end{array}$ & $\mathrm{AL}$ & 189 & 56 & 1 & 66 & Operational & 1969 & COAL & FRESH & ONCE THROUGH \\
\hline CRAWFORD & $\begin{array}{l}\text { COMMONWEALTH } \\
\text { EDISON CO }\end{array}$ & IL & 4110 & 867 & 7 & 239 & Operational & 1958 & COAL & FRESH & ONCE THROUGH \\
\hline CRAWFORD & $\begin{array}{l}\text { COMMONWEALTH } \\
\text { EDISON CO }\end{array}$ & IL & 4110 & 867 & 8 & 358 & Operational & 1961 & COAL & FRESH & ONCE THROUGH \\
\hline CRISP & $\begin{array}{l}\text { CRISP COUNTY } \\
\text { PWR COMM }\end{array}$ & GA & 4538 & 753 & 1 & 12.5 & Operational & 1958 & GAS & FRESH & ONCE THROUGH \\
\hline CRIST & GULF POWER CO & $\mathrm{FL}$ & 7801 & 641 & 4 & 93.8 & Operational & 1959 & COAL & BRACKISH & $\begin{array}{l}\text { COMBINATION (ONCE } \\
\text { THROUGH/HELPER TOWER) }\end{array}$ \\
\hline CRIST & GULF POWER CO & $\mathrm{FL}$ & 7801 & 641 & 5 & 93.8 & Operational & 1961 & COAL & BRACKISH & $\begin{array}{l}\text { COMBINATION (ONCE } \\
\text { THROUGH/HELPER TOWER) }\end{array}$ \\
\hline CRIST & GULF POWER CO & $\mathrm{FL}$ & 7801 & 641 & 3 & 37.5 & Operational & 1952 & GAS & BRACKISH & $\begin{array}{l}\text { COMBINATION (ONCE } \\
\text { THROUGH/HELPER TOWER) }\end{array}$ \\
\hline CRIST & GULF POWER CO & $\mathrm{FL}$ & 7801 & 641 & 2 & 28.1 & Operational & 1949 & GAS & BRACKISH & $\begin{array}{l}\text { COMBINATION (ONCE } \\
\text { THROUGH/HELPER TOWER) }\end{array}$ \\
\hline CRIST & GULF POWER CO & $\mathrm{FL}$ & 7801 & 641 & 1 & 28.1 & Operational & 1945 & GAS & BRACKISH & $\begin{array}{l}\text { COMBINATION (ONCE } \\
\text { THROUGH/HELPER TOWER) }\end{array}$ \\
\hline CROMBY & PECO ENERGY CO & PA & 14940 & 3159 & 1 & 188 & Operational & 1954 & COAL & FRESH & ONCE THROUGH \\
\hline CROMBY & PECO ENERGY CO & PA & 14940 & 3159 & 2 & 230 & Operational & 1955 & GAS & FRESH & ONCE THROUGH \\
\hline
\end{tabular}




\begin{tabular}{|c|c|c|c|c|c|c|c|c|c|c|c|}
\hline Plant Name & Utility Name & State & Utility/Pl & nt/Uni & $I D$ & $\boldsymbol{M W}$ & Status & Year & Fuel & Water & Cooling System \\
\hline $\begin{array}{l}\text { CRYSTAL } \\
\text { RIVER }\end{array}$ & $\begin{array}{l}\text { FLORIDA POWER } \\
\text { CORP }\end{array}$ & $\mathrm{FL}$ & 6455 & 628 & 2 & 524 & Operational & 1969 & COAL & SALINE & $\begin{array}{l}\text { COMBINATION (ONCE } \\
\text { THROUGH/HELPER TOWER) }\end{array}$ \\
\hline $\begin{array}{l}\text { CRYSTAL } \\
\text { RIVER }\end{array}$ & $\begin{array}{l}\text { FLORIDA POWER } \\
\text { CORP }\end{array}$ & $\mathrm{FL}$ & 6455 & 628 & 1 & 441 & Operational & 1966 & COAL & SALINE & $\begin{array}{l}\text { COMBINATION (ONCE } \\
\text { THROUGH/HELPER TOWER) }\end{array}$ \\
\hline $\begin{array}{l}\text { CRYSTAL } \\
\text { RIVER }\end{array}$ & $\begin{array}{l}\text { FLORIDA POWER } \\
\text { CORP }\end{array}$ & $\mathrm{FL}$ & 6455 & 628 & 3 & 890 & Operational & 1977 & UR & SALINE & $\begin{array}{l}\text { COMBINATION (ONCE } \\
\text { THROUGH/HELPER TOWER) }\end{array}$ \\
\hline CULLEY & $\begin{array}{l}\text { SOUTHERN INDIANA } \\
\text { GAS ELEC }\end{array}$ & IN & 17633 & 1012 & 1 & 46 & Operational & 1955 & COAL & FRESH & ONCE THROUGH \\
\hline CULLEY & $\begin{array}{l}\text { SOUTHERN INDIANA } \\
\text { GAS ELEC }\end{array}$ & IN & 17633 & 1012 & 2 & 99 & Operational & 1966 & COAL & FRESH & ONCE THROUGH \\
\hline CULLEY & $\begin{array}{l}\text { SOUTHERN INDIANA } \\
\text { GAS ELEC }\end{array}$ & IN & 17633 & 1012 & 3 & 265 & Operational & 1973 & COAL & FRESH & ONCE THROUGH \\
\hline CUMBERLAND & $\begin{array}{l}\text { TENNESSEE } \\
\text { VALLEY AUTH }\end{array}$ & $\mathrm{TN}$ & 18642 & 3399 & 2 & 1300 & Operational & 1973 & COAL & FRESH & ONCE THROUGH \\
\hline CUMBERLAND & $\begin{array}{l}\text { TENNESSEE } \\
\text { VALLEY AUTH }\end{array}$ & $\mathrm{TN}$ & 18642 & 3399 & 1 & 1300 & Operational & 1973 & COAL & FRESH & ONCE THROUGH \\
\hline CUTLER (FL) & $\begin{array}{l}\text { FLORIDA POWER \& } \\
\text { LIGHT CO }\end{array}$ & $\mathrm{FL}$ & 6452 & 610 & 5 & 75 & Operational & 1954 & GAS & SALINE & ONCE THROUGH \\
\hline CUTLER (FL) & $\begin{array}{l}\text { FLORIDA POWER \& } \\
\text { LIGHT CO }\end{array}$ & $\mathrm{FL}$ & 6452 & 610 & 6 & 162 & Operational & 1955 & GAS & SALINE & ONCE THROUGH \\
\hline DALE & $\begin{array}{l}\text { EAST KENTUCKY } \\
\text { POWER COOP }\end{array}$ & KY & 5580 & 1385 & 1 & 22 & Operational & 1954 & COAL & FRESH & ONCE THROUGH \\
\hline DALE & $\begin{array}{l}\text { EAST KENTUCKY } \\
\text { POWER COOP }\end{array}$ & KY & 5580 & 1385 & 2 & 22 & Operational & 1954 & COAL & FRESH & ONCE THROUGH \\
\hline DALE & $\begin{array}{l}\text { EAST KENTUCKY } \\
\text { POWER COOP }\end{array}$ & KY & 5580 & 1385 & 3 & 66 & Operational & 1957 & COAL & FRESH & ONCE THROUGH \\
\hline DALE & $\begin{array}{l}\text { EAST KENTUCKY } \\
\text { POWER COOP }\end{array}$ & KY & 5580 & 1385 & 4 & 66 & Operational & 1960 & COAL & FRESH & COMBINATION (NS) \\
\hline
\end{tabular}




\begin{tabular}{|c|c|c|c|c|c|c|c|c|c|c|c|}
\hline Plant Name & Utility Name & State & Utility/Pl & nt/Uni & $I D$ & $M W$ & Status & Year & Fuel & Water & Cooling System \\
\hline DALLMAN & $\begin{array}{l}\text { SPRINGFIELD WTR } \\
\text { LT \& PWR }\end{array}$ & IL & 17828 & 963 & 1 & 90.3 & Operational & 1968 & COAL & FRESH & ONCE THROUGH \\
\hline DALLMAN & $\begin{array}{l}\text { SPRINGFIELD WTR } \\
\text { LT \& PWR }\end{array}$ & IL & 17828 & 963 & 2 & 90.3 & Operational & 1971 & COAL & FRESH & ONCE THROUGH \\
\hline DALLMAN & $\begin{array}{l}\text { SPRINGFIELD WTR } \\
\text { LT \& PWR }\end{array}$ & IL & 17828 & 963 & 3 & 207 & Operational & 1978 & COAL & FRESH & ONCE THROUGH \\
\hline DAN RIVER & DUKE POWER CO & NC & 5416 & 2723 & 1 & 70 & Operational & 1949 & COAL & FRESH & ONCE THROUGH \\
\hline DAN RIVER & DUKE POWER CO & NC & 5416 & 2723 & 2 & 70 & Operational & 1950 & COAL & FRESH & ONCE THROUGH \\
\hline DAN RIVER & DUKE POWER CO & NC & 5416 & 2723 & 3 & 150 & Operational & 1955 & COAL & FRESH & ONCE THROUGH \\
\hline $\begin{array}{l}\text { DANSKAMMER } \\
\text { POINT }\end{array}$ & $\begin{array}{l}\text { CENTRAL HUDSON } \\
\text { GAS \& ELEC }\end{array}$ & NY & 3249 & 2480 & 2 & 73.5 & Operational & 1954 & GAS & FRESH & ONCE THROUGH \\
\hline $\begin{array}{l}\text { DANSKAMMER } \\
\text { POINT }\end{array}$ & $\begin{array}{l}\text { CENTRAL HUDSON } \\
\text { GAS \& ELEC }\end{array}$ & NY & 3249 & 2480 & 3 & 147 & Operational & 1959 & COAL & FRESH & ONCE THROUGH \\
\hline $\begin{array}{l}\text { DANSKAMMER } \\
\text { POINT }\end{array}$ & $\begin{array}{l}\text { CENTRAL HUDSON } \\
\text { GAS \& ELEC }\end{array}$ & NY & 3249 & 2480 & 1 & 72 & Operational & 1951 & GAS & FRESH & ONCE THROUGH \\
\hline $\begin{array}{l}\text { DANSKAMMER } \\
\text { POINT }\end{array}$ & $\begin{array}{l}\text { CENTRAL HUDSON } \\
\text { GAS \& ELEC }\end{array}$ & NY & 3249 & 2480 & 4 & 239 & Operational & 1967 & COAL & FRESH & ONCE THROUGH \\
\hline $\begin{array}{l}\text { DAVE } \\
\text { JOHNSTON }\end{array}$ & PACIFICORP & WY & 14356 & 4158 & 1 & 100 & Operational & 1959 & COAL & FRESH & $\begin{array}{l}\text { COMBINATION (ONCE } \\
\text { THROUGH/HELPER TOWER) }\end{array}$ \\
\hline $\begin{array}{l}\text { DAVE } \\
\text { JOHNSTON }\end{array}$ & PACIFICORP & WY & 14356 & 4158 & 2 & 100 & Operational & 1960 & COAL & FRESH & $\begin{array}{l}\text { COMBINATION (ONCE } \\
\text { THROUGH/HELPER TOWER) }\end{array}$ \\
\hline $\begin{array}{l}\text { DAVE } \\
\text { JOHNSTON }\end{array}$ & PACIFICORP & WY & 14356 & 4158 & 3 & 220 & Operational & 1964 & COAL & FRESH & $\begin{array}{l}\text { COMBINATION (ONCE } \\
\text { THROUGH/HELPER TOWER) }\end{array}$ \\
\hline DC COOK & $\begin{array}{l}\text { INDIANA MICHIGAN } \\
\text { POWER CO }\end{array}$ & MI & 9324 & 6000 & 1 & 1152 & Operational & 1975 & UR & FRESH & ONCE THROUGH \\
\hline
\end{tabular}




\begin{tabular}{|c|c|c|c|c|c|c|c|c|c|c|c|}
\hline Plant Name & Utility Name & State & Utility/Pl & $n t / U n i$ & $I D$ & $\boldsymbol{M W}$ & Status & Year & Fuel & Water & Cooling System \\
\hline DC COOK & $\begin{array}{l}\text { INDIANA MICHIGAN } \\
\text { POWER CO }\end{array}$ & MI & 9324 & 6000 & 2 & 1133 & Operational & 1978 & UR & FRESH & ONCE THROUGH \\
\hline DE KARN & $\begin{array}{l}\text { CONSUMERS } \\
\text { POWER CO }\end{array}$ & MI & 4254 & 1702 & 2 & 265 & Operational & 1961 & COAL & FRESH & ONCE THROUGH \\
\hline DE KARN & $\begin{array}{l}\text { CONSUMERS } \\
\text { POWER CO }\end{array}$ & MI & 4254 & 1702 & 1 & 265 & Operational & 1959 & COAL & FRESH & ONCE THROUGH \\
\hline DECKER & $\begin{array}{l}\text { AUSTIN ELECTRIC } \\
\text { DEPT }\end{array}$ & $\mathrm{TX}$ & 1015 & 3548 & 1 & 348 & Operational & 1972 & GAS & FRESH & ONCE THROUGH \\
\hline DECKER & $\begin{array}{l}\text { AUSTIN ELECTRIC } \\
\text { DEPT }\end{array}$ & TX & 1015 & 3548 & 2 & 440 & Operational & 1977 & GAS & FRESH & ONCE THROUGH \\
\hline DECORDOVA & $\begin{array}{l}\text { TEXAS UTILITIES } \\
\text { ELEC CO }\end{array}$ & $\mathrm{TX}$ & 44372 & 8063 & 1 & 799 & Operational & 1975 & GAS & FRESH & ONCE THROUGH \\
\hline $\begin{array}{l}\text { DEEPWATER } \\
(\mathrm{NJ})\end{array}$ & $\begin{array}{l}\text { DEEPWATER } \\
\text { OPERATING CO }\end{array}$ & $\mathrm{NJ}$ & 963 & 2384 & 4 & 53 & Operational & 1930 & OIL & BRACKISH & ONCE THROUGH \\
\hline $\begin{array}{l}\text { DEEPWATER } \\
(\mathrm{NJ})\end{array}$ & $\begin{array}{l}\text { DEEPWATER } \\
\text { OPERATING CO }\end{array}$ & NJ & 963 & 2384 & 6 & 73.5 & Operational & 1954 & COAL & BRACKISH & ONCE THROUGH \\
\hline $\begin{array}{l}\text { DEEPWATER } \\
(\mathrm{NJ})\end{array}$ & $\begin{array}{l}\text { DEEPWATER } \\
\text { OPERATING CO }\end{array}$ & NJ & 963 & 2384 & 1 & 81.6 & Operational & 1958 & GAS & BRACKISH & ONCE THROUGH \\
\hline $\begin{array}{l}\text { DEEPWATER } \\
\text { (TX) }\end{array}$ & $\begin{array}{l}\text { HOUSTON } \\
\text { LIGHTING \& POWER }\end{array}$ & $\mathrm{TX}$ & 8901 & 3461 & 9 & 188 & Operational & 1955 & GAS & BRACKISH & ONCE THROUGH \\
\hline DELAWARE & PECO ENERGY CO & PA & 14940 & 3160 & 7 & 156 & Operational & 1953 & OIL & FRESH & ONCE THROUGH \\
\hline DELAWARE & PECO ENERGY CO & PA & 14940 & 3160 & 8 & 156 & Operational & 1953 & OIL & FRESH & ONCE THROUGH \\
\hline DEVON & $\begin{array}{l}\text { CONNECTICUT } \\
\text { LIGHT \& POWER }\end{array}$ & CT & 4176 & 544 & 7 & 104 & Operational & 1954 & OIL & BRACKISH & ONCE THROUGH \\
\hline DEVON & $\begin{array}{l}\text { CONNECTICUT } \\
\text { LIGHT \& POWER }\end{array}$ & CT & 4176 & 544 & 8 & 104 & Operational & 1958 & OIL & BRACKISH & ONCE THROUGH \\
\hline
\end{tabular}




\begin{tabular}{|c|c|c|c|c|c|c|c|c|c|c|c|}
\hline Plant Name & Utility Name & State & Utility/Pl & $i t / U n i$ & $I D$ & $M W$ & Status & Year & Fuel & Water & Cooling System \\
\hline DH MITCHELL & $\begin{array}{l}\text { NO INDIANA PUBLIC } \\
\text { SERVICE }\end{array}$ & IN & 13756 & 996 & 4 & 138 & Operational & 1956 & COAL & FRESH & ONCE THROUGH \\
\hline DH MITCHELL & $\begin{array}{l}\text { NO INDIANA PUBLIC } \\
\text { SERVICE }\end{array}$ & IN & 13756 & 996 & 5 & 138 & Operational & 1959 & COAL & FRESH & ONCE THROUGH \\
\hline DH MITCHELL & $\begin{array}{l}\text { NO INDIANA PUBLIC } \\
\text { SERVICE }\end{array}$ & IN & 13756 & 996 & 6 & 138 & Operational & 1959 & COAL & FRESH & ONCE THROUGH \\
\hline DH MITCHELL & $\begin{array}{l}\text { NO INDIANA PUBLIC } \\
\text { SERVICE }\end{array}$ & IN & 13756 & 996 & 11 & 115 & Operational & 1970 & COAL & FRESH & ONCE THROUGH \\
\hline $\begin{array}{l}\text { DIABLO } \\
\text { CANYON }\end{array}$ & $\begin{array}{l}\text { PACIFIC GAS \& } \\
\text { ELEC CO }\end{array}$ & CA & 14328 & 6099 & 2 & 1164 & Operational & 1986 & UR & SALINE & ONCE THROUGH \\
\hline $\begin{array}{l}\text { DIABLO } \\
\text { CANYON }\end{array}$ & $\begin{array}{l}\text { PACIFIC GAS \& } \\
\text { ELEC CO }\end{array}$ & $\mathrm{CA}$ & 14328 & 6099 & 1 & 1137 & Operational & 1985 & UR & SALINE & ONCE THROUGH \\
\hline DICKERSON & $\begin{array}{l}\text { POTOMAC } \\
\text { ELECTRIC POWER } \\
n\end{array}$ & MD & 15270 & 1572 & 3 & 196 & Operational & 1962 & COAL & FRESH & ONCE THROUGH \\
\hline DICKERSON & $\begin{array}{l}\text { POTOMAC } \\
\text { ELECTRIC POWER } \\
n\end{array}$ & MD & 15270 & 1572 & 2 & 196 & Operational & 1960 & COAL & FRESH & ONCE THROUGH \\
\hline DICKERSON & $\begin{array}{l}\text { POTOMAC } \\
\text { ELECTRIC POWER } \\
n\end{array}$ & MD & 15270 & 1572 & 1 & 196 & Operational & 1959 & COAL & FRESH & ONCE THROUGH \\
\hline DOVER $(\mathrm{OH})$ & $\begin{array}{l}\text { DOVER }(\mathrm{OH}) \text { LIGHT } \\
\text { \& POWER }\end{array}$ & $\mathrm{OH}$ & 5336 & 2914 & 4 & 19.5 & Operational & 1968 & COAL & FRESH & ONCE THROUGH \\
\hline DUBUQUE & $\begin{array}{l}\text { INTERSTATE } \\
\text { POWER CO }\end{array}$ & IA & 9392 & 1046 & 2 & 15 & Operational & 1929 & COAL & FRESH & ONCE THROUGH \\
\hline DUBUQUE & $\begin{array}{l}\text { INTERSTATE } \\
\text { POWER CO }\end{array}$ & IA & 9392 & 1046 & 3 & 28.8 & Operational & 1952 & COAL & FRESH & ONCE THROUGH \\
\hline DUBUQUE & $\begin{array}{l}\text { INTERSTATE } \\
\text { POWER CO }\end{array}$ & IA & 9392 & 1046 & 4 & 37.5 & Operational & 1959 & COAL & FRESH & ONCE THROUGH \\
\hline DUNKIRK & $\begin{array}{l}\text { NIAGARA MOHAWK } \\
\text { POWER CORP }\end{array}$ & NY & 13573 & 2554 & 3 & 218 & Operational & 1959 & COAL & FRESH & ONCE THROUGH \\
\hline
\end{tabular}




\begin{tabular}{|c|c|c|c|c|c|c|c|c|c|c|c|}
\hline Plant Name & Utility Name & State & Utility/Pl & $n t / U n$ & $I D$ & $M W$ & Status & Year & Fuel & Water & Cooling System \\
\hline DUNKIRK & $\begin{array}{l}\text { NIAGARA MOHAWK } \\
\text { POWER CORP }\end{array}$ & NY & 13573 & 2554 & 4 & 218 & Operational & 1960 & COAL & FRESH & ONCE THROUGH \\
\hline DUNKIRK & $\begin{array}{l}\text { NIAGARA MOHAWK } \\
\text { POWER CORP }\end{array}$ & NY & 13573 & 2554 & 2 & 96 & Operational & 1950 & COAL & FRESH & ONCE THROUGH \\
\hline DUNKIRK & $\begin{array}{l}\text { NIAGARA MOHAWK } \\
\text { POWER CORP }\end{array}$ & NY & 13573 & 2554 & 1 & 96 & Operational & 1950 & COAL & FRESH & ONCE THROUGH \\
\hline $\begin{array}{l}\text { EAGLE } \\
\text { MOUNTAIN }\end{array}$ & $\begin{array}{l}\text { TEXAS UTILITIES } \\
\text { ELEC CO }\end{array}$ & $\mathrm{TX}$ & 44372 & 3489 & 2 & 188 & Operational & 1956 & GAS & FRESH & ONCE THROUGH \\
\hline $\begin{array}{l}\text { EAGLE } \\
\text { MOUNTAIN }\end{array}$ & $\begin{array}{l}\text { TEXAS UTILITIES } \\
\text { ELEC CO }\end{array}$ & $\mathrm{TX}$ & 44372 & 3489 & 3 & 396 & Operational & 1971 & GAS & FRESH & ONCE THROUGH \\
\hline $\begin{array}{l}\text { EAGLE } \\
\text { MOUNTAIN }\end{array}$ & $\begin{array}{l}\text { TEXAS UTILITIES } \\
\text { ELEC CO }\end{array}$ & $\mathrm{TX}$ & 44372 & 3489 & 1 & 123 & Operational & 1954 & GAS & FRESH & ONCE THROUGH \\
\hline EAST RIVER & $\begin{array}{l}\text { CONSOLIDATED } \\
\text { EDISON CO }\end{array}$ & NY & 4226 & 2493 & 7 & 200 & Operational & 1955 & GAS & SALINE & ONCE THROUGH \\
\hline EAST RIVER & $\begin{array}{l}\text { CONSOLIDATED } \\
\text { EDISON CO }\end{array}$ & NY & 4226 & 2493 & 6 & 156 & Operational & 1951 & GAS & SALINE & ONCE THROUGH \\
\hline EAST RIVER & $\begin{array}{l}\text { CONSOLIDATED } \\
\text { EDISON CO }\end{array}$ & NY & 4226 & 2493 & 5 & 156 & Operational & 1951 & OIL & SALINE & ONCE THROUGH \\
\hline EASTLAKE & $\begin{array}{l}\text { CLEVELAND ELEC } \\
\text { ILLUM CO }\end{array}$ & $\mathrm{OH}$ & 3755 & 2837 & 1 & 123 & Operational & 1953 & COAL & FRESH & ONCE THROUGH \\
\hline EASTLAKE & $\begin{array}{l}\text { CLEVELAND ELEC } \\
\text { ILLUM CO }\end{array}$ & $\mathrm{OH}$ & 3755 & 2837 & 2 & 123 & Operational & 1953 & COAL & FRESH & ONCE THROUGH \\
\hline EASTLAKE & $\begin{array}{l}\text { CLEVELAND ELEC } \\
\text { ILLUM CO }\end{array}$ & $\mathrm{OH}$ & 3755 & 2837 & 3 & 123 & Operational & 1954 & COAL & FRESH & ONCE THROUGH \\
\hline EASTLAKE & $\begin{array}{l}\text { CLEVELAND ELEC } \\
\text { ILLUM CO }\end{array}$ & $\mathrm{OH}$ & 3755 & 2837 & 4 & 208 & Operational & 1956 & COAL & FRESH & ONCE THROUGH \\
\hline EASTLAKE & $\begin{array}{l}\text { CLEVELAND ELEC } \\
\text { ILLUM CO }\end{array}$ & $\mathrm{OH}$ & 3755 & 2837 & 5 & 680 & Operational & 1972 & COAL & FRESH & ONCE THROUGH \\
\hline
\end{tabular}




\begin{tabular}{|c|c|c|c|c|c|c|c|c|c|c|c|}
\hline Plant Name & Utility Name & State & Utility/P & $n t / U n i$ & $I D$ & $M W$ & Status & Year & Fuel & Water & Cooling System \\
\hline EATON & $\begin{array}{l}\text { MISSISSIPPI } \\
\text { POWER CO }\end{array}$ & MS & 12686 & 2046 & 1 & 22.5 & Standby & 1945 & GAS & FRESH & ONCE THROUGH \\
\hline EATON & $\begin{array}{l}\text { MISSISSIPPI } \\
\text { POWER CO }\end{array}$ & MS & 12686 & 2046 & 2 & 22.5 & Standby & 1947 & GAS & FRESH & ONCE THROUGH \\
\hline EATON & $\begin{array}{l}\text { MISSISSIPPI } \\
\text { POWER CO }\end{array}$ & MS & 12686 & 2046 & 3 & 22.5 & Standby & 1949 & GAS & FRESH & ONCE THROUGH \\
\hline ECKERT & $\begin{array}{l}\text { LANSING BD } \\
\text { WATER \& LIGHT }\end{array}$ & MI & 10704 & 1831 & 1 & 50 & Operational & 1954 & COAL & FRESH & $\begin{array}{l}\text { COMBINATION (ONCE } \\
\text { THROUGH/HELPER TOWER) }\end{array}$ \\
\hline ECKERT & $\begin{array}{l}\text { LANSING BD } \\
\text { WATER \& LIGHT }\end{array}$ & MI & 10704 & 1831 & 2 & 46 & Operational & 1958 & COAL & FRESH & $\begin{array}{l}\text { COMBINATION (ONCE } \\
\text { THROUGH/HELPER TOWER) }\end{array}$ \\
\hline ECKERT & $\begin{array}{l}\text { LANSING BD } \\
\text { WATER \& LIGHT }\end{array}$ & MI & 10704 & 1831 & 3 & 50 & Operational & 1960 & COAL & FRESH & $\begin{array}{l}\text { COMBINATION (ONCE } \\
\text { THROUGH/HELPER TOWER) }\end{array}$ \\
\hline ECKERT & $\begin{array}{l}\text { LANSING BD } \\
\text { WATER \& LIGHT }\end{array}$ & MI & 10704 & 1831 & 4 & 75 & Operational & 1964 & COAL & FRESH & $\begin{array}{l}\text { COMBINATION (ONCE } \\
\text { THROUGH/HELPER TOWER) }\end{array}$ \\
\hline ECKERT & $\begin{array}{l}\text { LANSING BD } \\
\text { WATER \& LIGHT }\end{array}$ & MI & 10704 & 1831 & 5 & 75 & Operational & 1968 & COAL & FRESH & $\begin{array}{l}\text { COMBINATION (ONCE } \\
\text { THROUGH/HELPER TOWER) }\end{array}$ \\
\hline ECKERT & $\begin{array}{l}\text { LANSING BD } \\
\text { WATER \& LIGHT }\end{array}$ & MI & 10704 & 1831 & 6 & 75 & Operational & 1970 & COAL & FRESH & $\begin{array}{l}\text { COMBINATION (ONCE } \\
\text { THROUGH/HELPER TOWER) }\end{array}$ \\
\hline ED EDWARDS & $\begin{array}{l}\text { CENTRAL ILLINOIS } \\
\text { LIGHT CO }\end{array}$ & $\mathrm{IL}$ & 3252 & 856 & 1 & 136 & Operational & 1960 & COAL & FRESH & ONCE THROUGH \\
\hline ED EDWARDS & $\begin{array}{l}\text { CENTRAL ILLINOIS } \\
\text { LIGHT CO }\end{array}$ & IL & 3252 & 856 & 3 & 364 & Operational & 1972 & COAL & FRESH & ONCE THROUGH \\
\hline ED EDWARDS & $\begin{array}{l}\text { CENTRAL ILLINOIS } \\
\text { LIGHT CO }\end{array}$ & IL & 3252 & 856 & 2 & 281 & Operational & 1968 & COAL & FRESH & ONCE THROUGH \\
\hline EDDYSTONE & PECO ENERGY CO & PA & 14940 & 3161 & 1 & 354 & Operational & 1960 & COAL & FRESH & ONCE THROUGH \\
\hline EDDYSTONE & PECO ENERGY CO & PA & 14940 & 3161 & 2 & 354 & Operational & 1960 & COAL & FRESH & ONCE THROUGH \\
\hline
\end{tabular}




\begin{tabular}{|c|c|c|c|c|c|c|c|c|c|c|c|}
\hline Plant Name & Utility Name & State & Utility/Pl & $n t / U n i$ & $I D$ & $M W$ & Status & Year & Fuel & Water & Cooling System \\
\hline EDDYSTONE & PECO ENERGY CO & PA & 14940 & 3161 & 3 & 391 & Operational & 1974 & OIL & FRESH & ONCE THROUGH \\
\hline EDDYSTONE & PECO ENERGY CO & PA & 14940 & 3161 & 4 & 391 & Operational & 1976 & OIL & FRESH & ONCE THROUGH \\
\hline EDGE MOOR & $\begin{array}{l}\text { DELMARVA POWER } \\
\& \text { LIGHT CO }\end{array}$ & DE & 5027 & 593 & 4 & 177 & Operational & 1966 & COAL & BRACKISH & ONCE THROUGH \\
\hline EDGE MOOR & $\begin{array}{l}\text { DELMARVA POWER } \\
\& \text { LIGHT CO }\end{array}$ & DE & 5027 & 593 & 5 & 446 & Operational & 1973 & OIL & BRACKISH & ONCE THROUGH \\
\hline EDGE MOOR & $\begin{array}{l}\text { DELMARVA POWER } \\
\& \text { LIGHT CO }\end{array}$ & DE & 5027 & 593 & 3 & 75 & Operational & 1954 & COAL & BRACKISH & ONCE THROUGH \\
\hline $\begin{array}{l}\text { EDGEWATER } \\
(\mathrm{OH})\end{array}$ & OHIO EDISON CO & $\mathrm{OH}$ & 13998 & 2857 & 4 & 105 & Operational & 1957 & COAL & FRESH & ONCE THROUGH \\
\hline $\begin{array}{l}\text { EDGEWATER } \\
(\mathrm{OH})\end{array}$ & OHIO EDISON CO & $\mathrm{OH}$ & 13998 & 2857 & 2 & 25 & Standby & 1924 & COAL & FRESH & ONCE THROUGH \\
\hline $\begin{array}{l}\text { EDGEWATER } \\
(\mathrm{Wl}\end{array}$ & $\begin{array}{l}\text { WISCONSIN } \\
\text { POWER \& LIGHT }\end{array}$ & WI & 20856 & 4050 & 3 & 66 & Operational & 1951 & COAL & FRESH & ONCE THROUGH \\
\hline $\begin{array}{l}\text { EDGEWATER } \\
(\mathrm{WI})\end{array}$ & $\begin{array}{l}\text { WISCONSIN } \\
\text { POWER \& LIGHT }\end{array}$ & WI & 20856 & 4050 & 4 & 351 & Operational & 1969 & COAL & FRESH & ONCE THROUGH \\
\hline $\begin{array}{l}\text { EDWARDSPOR } \\
\text { T }\end{array}$ & PSI ENERGY INC & IN & 15470 & 1004 & 8 & 69 & Operational & 1951 & COAL & FRESH & ONCE THROUGH \\
\hline $\begin{array}{l}\text { EDWARDSPOR } \\
T\end{array}$ & PSI ENERGY INC & IN & 15470 & 1004 & 6 & 35 & Operational & 1944 & OIL & FRESH & ONCE THROUGH \\
\hline $\begin{array}{l}\text { EDWARDSPOR } \\
\text { T }\end{array}$ & PSI ENERGY INC & IN & 15470 & 1004 & 7 & 40.3 & Operational & 1949 & COAL & FRESH & ONCE THROUGH \\
\hline EF BARRETT & $\begin{array}{l}\text { LONG ISLAND } \\
\text { LIGHTING CO }\end{array}$ & NY & 11172 & 2511 & 2 & 188 & Operational & 1963 & GAS & SALINE & ONCE THROUGH \\
\hline EF BARRETT & $\begin{array}{l}\text { LONG ISLAND } \\
\text { LIGHTING CO }\end{array}$ & NY & 11172 & 2511 & 1 & 188 & Operational & 1956 & GAS & SALINE & ONCE THROUGH \\
\hline
\end{tabular}




\begin{tabular}{|c|c|c|c|c|c|c|c|c|c|c|c|}
\hline Plant Name & Utility Name & State & Utility/Pl & $n t / U n i$ & $I D$ & $\boldsymbol{M W}$ & Status & Year & Fuel & Water & Cooling System \\
\hline EL SEGUNDO & $\begin{array}{l}\text { SOUTHERN CALIF } \\
\text { EDISON CO }\end{array}$ & $\mathrm{CA}$ & 17609 & 330 & 3 & 342 & Operational & 1964 & GAS & SALINE & ONCE THROUGH \\
\hline EL SEGUNDO & $\begin{array}{l}\text { SOUTHERN CALIF } \\
\text { EDISON CO }\end{array}$ & $\mathrm{CA}$ & 17609 & 330 & 4 & 342 & Operational & 1965 & GAS & SALINE & ONCE THROUGH \\
\hline EL SEGUNDO & $\begin{array}{l}\text { SOUTHERN CALIF } \\
\text { EDISON CO }\end{array}$ & $\mathrm{CA}$ & 17609 & 330 & 2 & 156 & Operational & 1956 & GAS & SALINE & ONCE THROUGH \\
\hline EL SEGUNDO & $\begin{array}{l}\text { SOUTHERN CALIF } \\
\text { EDISON CO }\end{array}$ & $\mathrm{CA}$ & 17609 & 330 & 1 & 156 & Operational & 1955 & GAS & SALINE & ONCE THROUGH \\
\hline ELK RIVER & $\begin{array}{l}\text { UNITED POWER } \\
\text { ASSOCIATION }\end{array}$ & $\mathrm{MN}$ & 19514 & 2039 & 2 & 11.5 & Operational & 1951 & RDF & FRESH & ONCE THROUGH \\
\hline ELK RIVER & $\begin{array}{l}\text { UNITED POWER } \\
\text { ASSOCIATION }\end{array}$ & $\mathrm{MN}$ & 19514 & 2039 & 3 & 22.5 & Operational & 1959 & RDF & FRESH & ONCE THROUGH \\
\hline ELK RIVER & $\begin{array}{l}\text { UNITED POWER } \\
\text { ASSOCIATION }\end{array}$ & MN & 19514 & 2039 & 1 & 11.5 & Operational & 1951 & RDF & FRESH & ONCE THROUGH \\
\hline ELMER SMITH & $\begin{array}{l}\text { OWENSBORO } \\
\text { MUNICIPAL UTIL }\end{array}$ & KY & 14268 & 1374 & 1 & 151 & Operational & 1964 & COAL & FRESH & ONCE THROUGH \\
\hline ELMER SMITH & $\begin{array}{l}\text { OWENSBORO } \\
\text { MUNICIPAL UTIL }\end{array}$ & KY & 14268 & 1374 & 2 & 265 & Operational & 1974 & COAL & FRESH & ONCE THROUGH \\
\hline ELRAMA & $\begin{array}{l}\text { DUQUESNE LIGHT } \\
\text { CO }\end{array}$ & PA & 5487 & 3098 & 3 & 125 & Operational & 1954 & COAL & FRESH & ONCE THROUGH \\
\hline ELRAMA & $\begin{array}{l}\text { DUQUESNE LIGHT } \\
\text { CO }\end{array}$ & PA & 5487 & 3098 & 4 & 185 & Operational & 1960 & COAL & FRESH & ONCE THROUGH \\
\hline ELRAMA & $\begin{array}{l}\text { DUQUESNE LIGHT } \\
\text { CO }\end{array}$ & PA & 5487 & 3098 & 1 & 100 & Operational & 1952 & COAL & FRESH & ONCE THROUGH \\
\hline ELRAMA & $\begin{array}{l}\text { DUQUESNE LIGHT } \\
\text { CO }\end{array}$ & PA & 5487 & 3098 & 2 & 100 & Operational & 1953 & COAL & FRESH & ONCE THROUGH \\
\hline ENCINA & $\begin{array}{l}\text { SAN DIEGO GAS \& } \\
\text { ELEC CO }\end{array}$ & $\mathrm{CA}$ & 16609 & 302 & 1 & 110 & Operational & 1954 & GAS & SALINE & ONCE THROUGH \\
\hline
\end{tabular}




\begin{tabular}{|c|c|c|c|c|c|c|c|c|c|c|c|}
\hline Plant Name & Utility Name & State & Utility/Pla & $n t / U n i$ & $I D$ & $M W$ & Status & Year & Fuel & Water & Cooling System \\
\hline ENCINA & $\begin{array}{l}\text { SAN DIEGO GAS \& } \\
\text { ELEC CO }\end{array}$ & $\mathrm{CA}$ & 16609 & 302 & 2 & 110 & Operational & 1956 & GAS & SALINE & ONCE THROUGH \\
\hline ENCINA & $\begin{array}{l}\text { SAN DIEGO GAS \& } \\
\text { ELEC CO }\end{array}$ & $\mathrm{CA}$ & 16609 & 302 & 3 & 110 & Operational & 1958 & GAS & SALINE & ONCE THROUGH \\
\hline ENCINA & $\begin{array}{l}\text { SAN DIEGO GAS \& } \\
\text { ELEC CO }\end{array}$ & $\mathrm{CA}$ & 16609 & 302 & 4 & 306 & Operational & 1973 & GAS & SALINE & ONCE THROUGH \\
\hline ENCINA & $\begin{array}{l}\text { SAN DIEGO GAS \& } \\
\text { ELEC CO }\end{array}$ & CA & 16609 & 302 & 5 & 346 & Operational & 1978 & GAS & SALINE & ONCE THROUGH \\
\hline ESCANABA & $\begin{array}{l}\text { UPPER PENINSULA } \\
\text { POWER CO }\end{array}$ & MI & 19578 & 1771 & 1 & 11.5 & Operational & 1958 & COAL & FRESH & ONCE THROUGH \\
\hline ESCANABA & $\begin{array}{l}\text { UPPER PENINSULA } \\
\text { POWER CO }\end{array}$ & $\mathrm{MI}$ & 19578 & 1771 & 2 & 11.5 & Operational & 1958 & COAL & FRESH & ONCE THROUGH \\
\hline EW STOUT & $\begin{array}{l}\text { INDIANAPOLIS } \\
\text { POWER \& LT }\end{array}$ & IN & 9273 & 990 & 4 & 37.5 & Standby & 1947 & OIL & FRESH & ONCE THROUGH \\
\hline EW STOUT & $\begin{array}{l}\text { INDIANAPOLIS } \\
\text { POWER \& LT }\end{array}$ & IN & 9273 & 990 & 5 & 114 & Operational & 1958 & COAL & FRESH & $\begin{array}{l}\text { COMBINATION (ONCE } \\
\text { THROUGH/HELPER TOWER) }\end{array}$ \\
\hline EW STOUT & $\begin{array}{l}\text { INDIANAPOLIS } \\
\text { POWER \& LT }\end{array}$ & IN & 9273 & 990 & 3 & 37.5 & Standby & 1941 & OIL & FRESH & ONCE THROUGH \\
\hline EW STOUT & $\begin{array}{l}\text { INDIANAPOLIS } \\
\text { POWER \& LT }\end{array}$ & IN & 9273 & 990 & 6 & 114 & Operational & 1961 & COAL & FRESH & $\begin{array}{l}\text { COMBINATION (ONCE } \\
\text { THROUGH/HELPER TOWER) }\end{array}$ \\
\hline FAIRBURY & $\begin{array}{l}\text { FAIRBURY LIGHT \& } \\
\text { WTR DEPT }\end{array}$ & NE & 6132 & 2236 & 2 & 2.5 & Standby & 1938 & GAS & FRESH & ONCE THROUGH \\
\hline $\begin{array}{l}\text { FAR } \\
\text { ROCKAWAY }\end{array}$ & $\begin{array}{l}\text { LONG ISLAND } \\
\text { LIGHTING CO }\end{array}$ & NY & 11172 & 2513 & 4 & 114 & Operational & 1953 & GAS & SALINE & ONCE THROUGH \\
\hline FE FAIR & $\begin{array}{l}\text { CENTRAL IOWA } \\
\text { POWER COOP }\end{array}$ & IA & 3258 & 1218 & 1 & 25 & Operational & 1960 & COAL & FRESH & ONCE THROUGH \\
\hline FE FAIR & $\begin{array}{l}\text { CENTRAL IOWA } \\
\text { POWER COOP }\end{array}$ & IA & 3258 & 1218 & 2 & 37.5 & Operational & 1967 & COAL & FRESH & ONCE THROUGH \\
\hline
\end{tabular}




\begin{tabular}{|c|c|c|c|c|c|c|c|c|c|c|c|}
\hline Plant Name & Utility Name & State & Utility/Pl & $n t / U n i$ & $I D$ & $\boldsymbol{M W}$ & Status & Year & Fuel & Water & Cooling System \\
\hline FISK & $\begin{array}{l}\text { COMMONWEALTH } \\
\text { EDISON CO }\end{array}$ & IL & 4110 & 886 & 19 & 374 & Operational & 1959 & COAL & FRESH & ONCE THROUGH \\
\hline FITZHUGH & $\begin{array}{l}\text { ARKANSAS ELEC } \\
\text { COOP CORP }\end{array}$ & AR & 807 & 201 & 1 & 60 & Operational & 1963 & GAS & FRESH & ONCE THROUGH \\
\hline FITZPATRICK & $\begin{array}{l}\text { NEW YORK POWER } \\
\text { AUTHORITY }\end{array}$ & NY & 15296 & 6110 & 1 & 883 & Operational & 1975 & UR & FRESH & ONCE THROUGH \\
\hline $\begin{array}{l}\text { FORT } \\
\text { CALHOUN }\end{array}$ & $\begin{array}{l}\text { OMAHA PUBLIC } \\
\text { POWER DIST }\end{array}$ & NE & 14127 & 2289 & 1 & 502 & Operational & 1974 & UR & FRESH & ONCE THROUGH \\
\hline FORT MYERS & $\begin{array}{l}\text { FLORIDA POWER \& } \\
\text { LIGHT CO }\end{array}$ & $\mathrm{FL}$ & 6452 & 612 & 1 & 156 & Operational & 1958 & OIL & BRACKISH & ONCE THROUGH \\
\hline FORT MYERS & $\begin{array}{l}\text { FLORIDA POWER \& } \\
\text { LIGHT CO }\end{array}$ & $\mathrm{FL}$ & 6452 & 612 & 2 & 402 & Operational & 1969 & OIL & BRACKISH & ONCE THROUGH \\
\hline $\begin{array}{l}\text { FORT } \\
\text { PHANTOM }\end{array}$ & $\begin{array}{l}\text { WEST TEXAS UTIL } \\
\text { CO }\end{array}$ & TX & 20404 & 4938 & 1 & 157 & Operational & 1974 & GAS & FRESH & ONCE THROUGH \\
\hline $\begin{array}{l}\text { FORT } \\
\text { PHANTOM }\end{array}$ & $\begin{array}{l}\text { WEST TEXAS UTIL } \\
\text { CO }\end{array}$ & TX & 20404 & 4938 & 2 & 207 & Operational & 1977 & GAS & FRESH & ONCE THROUGH \\
\hline $\begin{array}{l}\text { FOURTH } \\
\text { AVENUE }\end{array}$ & AUSTIN UTILITIES & $\mathrm{MN}$ & 1009 & 1961 & 3 & 8 & Operational & 1949 & GAS & FRESH & ONCE THROUGH \\
\hline FOX LAKE & $\begin{array}{l}\text { INTERSTATE } \\
\text { POWER CO }\end{array}$ & $\mathrm{MN}$ & 9392 & 1888 & 3 & 81.6 & Operational & 1962 & GAS & FRESH & ONCE THROUGH \\
\hline FOX LAKE & $\begin{array}{l}\text { INTERSTATE } \\
\text { POWER CO }\end{array}$ & $\mathrm{MN}$ & 9392 & 1888 & 2 & 11.5 & Operational & 1951 & GAS & FRESH & ONCE THROUGH \\
\hline FOX LAKE & $\begin{array}{l}\text { INTERSTATE } \\
\text { POWER CO }\end{array}$ & $\mathrm{MN}$ & 9392 & 1888 & 1 & 11.5 & Operational & 1950 & GAS & FRESH & ONCE THROUGH \\
\hline FR PHILLIPS & $\begin{array}{l}\text { DUQUESNE LIGHT } \\
\text { CO }\end{array}$ & PA & 5487 & 3099 & 1 & 69 & Standby & 1943 & COAL & FRESH & ONCE THROUGH \\
\hline FR PHILLIPS & $\begin{array}{l}\text { DUQUESNE LIGHT } \\
\text { CO }\end{array}$ & PA & 5487 & 3099 & 2 & 81.3 & Standby & 1949 & COAL & FRESH & ONCE THROUGH \\
\hline
\end{tabular}




\begin{tabular}{|c|c|c|c|c|c|c|c|c|c|c|c|}
\hline Plant Name & Utility Name & State & Utility/Pl & tt/Uni & $D$ & $M W$ & Status & Year & Fuel & Water & Cooling System \\
\hline FR PHILLIPS & $\begin{array}{l}\text { DUQUESNE LIGHT } \\
\text { CO }\end{array}$ & PA & 5487 & 3099 & 3 & 81.3 & Standby & 1950 & COAL & FRESH & ONCE THROUGH \\
\hline FR PHILLIPS & $\begin{array}{l}\text { DUQUESNE LIGHT } \\
\text { CO }\end{array}$ & PA & 5487 & 3099 & 4 & 180 & Operational & 1956 & COAL & FRESH & ONCE THROUGH \\
\hline FRANK BIRD & $\begin{array}{l}\text { MONTANA POWER } \\
\text { CO }\end{array}$ & MT & 12825 & 2187 & 1 & 69 & Standby & 1951 & GAS & FRESH & ONCE THROUGH \\
\hline $\begin{array}{l}\text { FRENCH } \\
\text { ISLAND }\end{array}$ & $\begin{array}{l}\text { NORTHERN STATES } \\
\text { POWER CO }\end{array}$ & WI & 13781 & 4005 & 1 & 15.3 & Operational & 1940 & WOOD & FRESH & ONCE THROUGH \\
\hline $\begin{array}{l}\text { FRENCH } \\
\text { ISLAND }\end{array}$ & $\begin{array}{l}\text { NORTHERN STATES } \\
\text { POWER CO }\end{array}$ & WI & 13781 & 4005 & 2 & 12.5 & Operational & 1948 & WOOD & FRESH & ONCE THROUGH \\
\hline $\begin{array}{l}\text { GADSDEN } \\
\text { NEW }\end{array}$ & $\begin{array}{l}\text { ALABAMA POWER } \\
\text { CO }\end{array}$ & $A L$ & 195 & 7 & 1 & 69 & Operational & 1949 & COAL & FRESH & ONCE THROUGH \\
\hline $\begin{array}{l}\text { GADSDEN } \\
\text { NEW }\end{array}$ & $\begin{array}{l}\text { ALABAMA POWER } \\
\text { CO }\end{array}$ & $\mathrm{AL}$ & 195 & 7 & 2 & 69 & Operational & 1949 & COAL & FRESH & ONCE THROUGH \\
\hline GALLAGHER & PSI ENERGY INC & IN & 15470 & 1008 & 3 & 150 & Operational & 1960 & COAL & FRESH & ONCE THROUGH \\
\hline GALLAGHER & PSI ENERGY INC & IN & 15470 & 1008 & 4 & 150 & Operational & 1961 & COAL & FRESH & ONCE THROUGH \\
\hline GALLAGHER & PSI ENERGY INC & IN & 15470 & 1008 & 1 & 150 & Operational & 1959 & COAL & FRESH & ONCE THROUGH \\
\hline GALLAGHER & PSI ENERGY INC & IN & 15470 & 1008 & 2 & 150 & Operational & 1958 & COAL & FRESH & ONCE THROUGH \\
\hline GALLATIN & $\begin{array}{l}\text { TENNESSEE } \\
\text { VALLEY AUTH }\end{array}$ & TN & 18642 & 3403 & 2 & 300 & Operational & 1957 & COAL & FRESH & ONCE THROUGH \\
\hline GALLATIN & $\begin{array}{l}\text { TENNESSEE } \\
\text { VALLEY AUTH }\end{array}$ & $\mathrm{TN}$ & 18642 & 3403 & 3 & 328 & Operational & 1959 & COAL & FRESH & ONCE THROUGH \\
\hline GALLATIN & $\begin{array}{l}\text { TENNESSEE } \\
\text { VALLEY AUTH }\end{array}$ & $\mathrm{TN}$ & 18642 & 3403 & 4 & 328 & Operational & 1959 & COAL & FRESH & ONCE THROUGH \\
\hline
\end{tabular}




\begin{tabular}{|c|c|c|c|c|c|c|c|c|c|c|c|}
\hline Plant Name & Utility Name & State & Utility/Pl & $n t / U n i$ & $I D$ & $M W$ & Status & Year & Fuel & Water & Cooling System \\
\hline GALLATIN & $\begin{array}{l}\text { TENNESSEE } \\
\text { VALLEY AUTH }\end{array}$ & $\mathrm{TN}$ & 18642 & 3403 & 1 & 300 & Operational & 1956 & COAL & FRESH & ONCE THROUGH \\
\hline GANNON & $\begin{array}{l}\text { TAMPA ELECTRIC } \\
\text { CO }\end{array}$ & $\mathrm{FL}$ & 18454 & 646 & 3 & 180 & Operational & 1960 & COAL & SALINE & ONCE THROUGH \\
\hline GANNON & $\begin{array}{l}\text { TAMPA ELECTRIC } \\
\text { CO }\end{array}$ & $\mathrm{FL}$ & 18454 & 646 & 6 & 414 & Operational & 1967 & COAL & SALINE & ONCE THROUGH \\
\hline GANNON & $\begin{array}{l}\text { TAMPA ELECTRIC } \\
\text { CO }\end{array}$ & $\mathrm{FL}$ & 18454 & 646 & 4 & 188 & Operational & 1963 & COAL & SALINE & ONCE THROUGH \\
\hline GANNON & $\begin{array}{l}\text { TAMPA ELECTRIC } \\
\text { CO }\end{array}$ & $\mathrm{FL}$ & 18454 & 646 & 2 & 125 & Operational & 1958 & COAL & SALINE & ONCE THROUGH \\
\hline GANNON & $\begin{array}{l}\text { TAMPA ELECTRIC } \\
\text { CO }\end{array}$ & $\mathrm{FL}$ & 18454 & 646 & 1 & 125 & Operational & 1957 & COAL & SALINE & ONCE THROUGH \\
\hline GANNON & $\begin{array}{l}\text { TAMPA ELECTRIC } \\
\text { CO }\end{array}$ & $\mathrm{FL}$ & 18454 & 646 & 5 & 239 & Operational & 1965 & COAL & SALINE & ONCE THROUGH \\
\hline GASTON (AL) & $\begin{array}{l}\text { ALABAMA POWER } \\
\text { CO }\end{array}$ & $A L$ & 195 & 26 & 1 & 272 & Operational & 1960 & COAL & FRESH & ONCE THROUGH \\
\hline GASTON (AL) & $\begin{array}{l}\text { ALABAMA POWER } \\
\text { CO }\end{array}$ & $\mathrm{AL}$ & 195 & 26 & 2 & 272 & Operational & 1960 & COAL & FRESH & ONCE THROUGH \\
\hline GASTON (AL) & $\begin{array}{l}\text { ALABAMA POWER } \\
\text { CO }\end{array}$ & $\mathrm{AL}$ & 195 & 26 & 3 & 272 & Operational & 1961 & COAL & FRESH & ONCE THROUGH \\
\hline GASTON (AL) & $\begin{array}{l}\text { ALABAMA POWER } \\
\text { CO }\end{array}$ & $\mathrm{AL}$ & 195 & 26 & 4 & 245 & Operational & 1962 & COAL & FRESH & ONCE THROUGH \\
\hline GENOA THREE & $\begin{array}{l}\text { DAIRYLAND POWER } \\
\text { COOP }\end{array}$ & WI & 4716 & 4143 & 1 & 346 & Operational & 1969 & COAL & FRESH & ONCE THROUGH \\
\hline GEORGE NEAL & $\begin{array}{l}\text { MIDWEST POWER } \\
\text { SYSTEMS }\end{array}$ & $\mathrm{IA}$ & 9435 & 1091 & 1 & 147 & Operational & 1964 & COAL & FRESH & ONCE THROUGH \\
\hline GEORGE NEAL & $\begin{array}{l}\text { MIDWEST POWER } \\
\text { SYSTEMS }\end{array}$ & $\mathrm{IA}$ & 9435 & 1091 & 2 & 349 & Operational & 1972 & COAL & FRESH & ONCE THROUGH \\
\hline
\end{tabular}




\begin{tabular}{|c|c|c|c|c|c|c|c|c|c|c|c|}
\hline Plant Name & Utility Name & State & Utility/Pl & $n t / U n i$ & $I D$ & $M W$ & Status & Year & Fuel & Water & Cooling System \\
\hline GEORGE NEAL & $\begin{array}{l}\text { MIDWEST POWER } \\
\text { SYSTEMS }\end{array}$ & IA & 9435 & 1091 & 3 & 550 & Operational & 1976 & COAL & FRESH & ONCE THROUGH \\
\hline GEORGE NEAL & $\begin{array}{l}\text { MIDWEST POWER } \\
\text { SYSTEMS }\end{array}$ & IA & 9435 & 1091 & 4 & 640 & Operational & 1979 & COAL & FRESH & ONCE THROUGH \\
\hline $\begin{array}{l}\text { GEORGE NEAL } \\
\text { NORTH }\end{array}$ & $\begin{array}{l}\text { MIDWEST POWER } \\
\text { SYSTEMS }\end{array}$ & IA & 9435 & 1091 & 3 & 550 & Operational & 1975 & COAL & FRESH & ONCE THROUGH \\
\hline $\begin{array}{l}\text { GEORGE NEAL } \\
\text { NORTH }\end{array}$ & $\begin{array}{l}\text { MIDWEST POWER } \\
\text { SYSTEMS }\end{array}$ & IA & 9435 & 1091 & 1 & 147 & Operational & 1964 & COAL & FRESH & ONCE THROUGH \\
\hline $\begin{array}{l}\text { GEORGE NEAL } \\
\text { NORTH }\end{array}$ & $\begin{array}{l}\text { MIDWEST POWER } \\
\text { SYSTEMS }\end{array}$ & IA & 9435 & 1091 & 2 & 349 & Operational & 1972 & COAL & FRESH & ONCE THROUGH \\
\hline $\begin{array}{l}\text { GEORGE NEAL } \\
\text { SOUTH }\end{array}$ & $\begin{array}{l}\text { MIDWEST POWER } \\
\text { SYSTEMS }\end{array}$ & IA & 9435 & 1091 & 4 & 640 & Operational & 1979 & COAL & FRESH & ONCE THROUGH \\
\hline $\begin{array}{l}\text { GERALD } \\
\text { GENTLEMAN }\end{array}$ & $\begin{array}{l}\text { NEBRASKA PUBLIC } \\
\text { POWER DIS }\end{array}$ & NE & 13337 & 6077 & 2 & 628 & Operational & 1982 & COAL & FRESH & ONCE THROUGH \\
\hline $\begin{array}{l}\text { GERALD } \\
\text { GENTLEMAN }\end{array}$ & $\begin{array}{l}\text { NEBRASKA PUBLIC } \\
\text { POWER DIS }\end{array}$ & NE & 13337 & 6077 & 1 & 681 & Operational & 1979 & COAL & FRESH & ONCE THROUGH \\
\hline GILBERT & $\begin{array}{l}\text { JERSEY CENT } \\
\text { POWER \& LIGHT }\end{array}$ & NJ & 9726 & 2393 & 1 & 11.8 & Operational & 1930 & OIL & FRESH & ONCE THROUGH \\
\hline GILBERT & $\begin{array}{l}\text { JERSEY CENT } \\
\text { POWER \& LIGHT }\end{array}$ & NJ & 9726 & 2393 & 2 & 45.3 & Operational & 1930 & OIL & FRESH & ONCE THROUGH \\
\hline GILBERT & $\begin{array}{l}\text { JERSEY CENT } \\
\text { POWER \& LIGHT }\end{array}$ & NJ & 9726 & 2393 & 3 & 69 & Operational & 1949 & OIL & FRESH & ONCE THROUGH \\
\hline GINNA & $\begin{array}{l}\text { ROCHESTER GAS \& } \\
\text { ELEC CORP }\end{array}$ & NY & 16183 & 6122 & 1 & 490 & Operational & 1970 & UR & FRESH & ONCE THROUGH \\
\hline GLEN LYN & $\begin{array}{l}\text { APPALACHIAN } \\
\text { POWER CO }\end{array}$ & VA & 733 & 3776 & 6 & 238 & Operational & 1957 & COAL & FRESH & ONCE THROUGH \\
\hline GLEN LYN & $\begin{array}{l}\text { APPALACHIAN } \\
\text { POWER CO }\end{array}$ & VA & 733 & 3776 & 5 & 100 & Operational & 1944 & COAL & FRESH & ONCE THROUGH \\
\hline
\end{tabular}




\begin{tabular}{|c|c|c|c|c|c|c|c|c|c|c|c|}
\hline Plant Name & Utility Name & State & Utility/Pl & $n t / U n i$ & $I D$ & $M W$ & Status & Year & Fuel & Water & Cooling System \\
\hline $\begin{array}{l}\text { GLENWOOD } \\
\text { (NY) }\end{array}$ & $\begin{array}{l}\text { LONG ISLAND } \\
\text { LIGHTING CO }\end{array}$ & NY & 11172 & 2514 & 4 & 114 & Operational & 1952 & GAS & BRACKISH & ONCE THROUGH \\
\hline $\begin{array}{l}\text { GLENWOOD } \\
\text { (NY) }\end{array}$ & $\begin{array}{l}\text { LONG ISLAND } \\
\text { LIGHTING CO }\end{array}$ & NY & 11172 & 2514 & 5 & 114 & Operational & 1954 & GAS & BRACKISH & ONCE THROUGH \\
\hline GORGAS TWO & $\begin{array}{l}\text { ALABAMA POWER } \\
\text { CO }\end{array}$ & $A L$ & 195 & 8 & 9 & 190 & Operational & 1958 & COAL & FRESH & ONCE THROUGH \\
\hline GORGAS TWO & $\begin{array}{l}\text { ALABAMA POWER } \\
\text { CO }\end{array}$ & $A L$ & 195 & 8 & 10 & 789 & Operational & 1972 & COAL & FRESH & ONCE THROUGH \\
\hline GORGAS TWO & $\begin{array}{l}\text { ALABAMA POWER } \\
\text { CO }\end{array}$ & $A L$ & 195 & 8 & 6 & 125 & Operational & 1951 & COAL & FRESH & ONCE THROUGH \\
\hline GORGAS TWO & $\begin{array}{l}\text { ALABAMA POWER } \\
\text { CO }\end{array}$ & $\mathrm{AL}$ & 195 & 8 & 7 & 125 & Operational & 1952 & COAL & FRESH & ONCE THROUGH \\
\hline GORGAS TWO & $\begin{array}{l}\text { ALABAMA POWER } \\
\text { CO }\end{array}$ & $A L$ & 195 & 8 & 8 & 188 & Operational & 1956 & COAL & FRESH & ONCE THROUGH \\
\hline GOUDEY & $\begin{array}{l}\text { NEW YORK STATE } \\
\text { ELEC \& GAS }\end{array}$ & NY & 13511 & 2526 & 8 & 75 & Operational & 1951 & COAL & FRESH & ONCE THROUGH \\
\hline GOUDEY & $\begin{array}{l}\text { NEW YORK STATE } \\
\text { ELEC \& GAS }\end{array}$ & NY & 13511 & 2526 & 7 & 43.8 & Operational & 1943 & COAL & FRESH & ONCE THROUGH \\
\hline $\begin{array}{l}\text { GOULD } \\
\text { STREET }\end{array}$ & $\begin{array}{l}\text { BALTIMORE GAS \& } \\
\text { ELEC CO }\end{array}$ & MD & 1167 & 1553 & 3 & 104 & Operational & 1952 & OIL & BRACKISH & ONCE THROUGH \\
\hline GRAHAM & $\begin{array}{l}\text { TEXAS UTILITIES } \\
\text { ELEC CO }\end{array}$ & TX & 44372 & 3490 & 1 & 248 & Operational & 1960 & GAS & FRESH & ONCE THROUGH \\
\hline GRAHAM & $\begin{array}{l}\text { TEXAS UTILITIES } \\
\text { ELEC CO }\end{array}$ & TX & 44372 & 3490 & 2 & 387 & Operational & 1969 & GAS & FRESH & ONCE THROUGH \\
\hline GRAINGER & $\begin{array}{l}\text { SOUTH CAROLINA } \\
\text { PUB SERV }\end{array}$ & SC & 17543 & 3317 & 1 & 81.6 & Operational & 1966 & COAL & FRESH & $\begin{array}{l}\text { COMBINATION (ONCE } \\
\text { THROUGH/HELPER POND) }\end{array}$ \\
\hline GRAINGER & $\begin{array}{l}\text { SOUTH CAROLINA } \\
\text { PUB SERV }\end{array}$ & SC & 17543 & 3317 & 2 & 81.6 & Operational & 1966 & COAL & FRESH & $\begin{array}{l}\text { COMBINATION (ONCE } \\
\text { THROUGH/HELPER POND) }\end{array}$ \\
\hline
\end{tabular}




\begin{tabular}{|c|c|c|c|c|c|c|c|c|c|c|c|}
\hline Plant Name & Utility Name & State & Utility/Pl & $n t / U n i$ & $I D$ & $M W$ & Status & Year & Fuel & Water & Cooling System \\
\hline $\begin{array}{l}\text { GRAND } \\
\text { TOWER }\end{array}$ & $\begin{array}{l}\text { CENT ILLINOIS } \\
\text { PUBLIC SERV }\end{array}$ & IL & 3253 & 862 & 3 & 85.7 & Operational & 1951 & COAL & FRESH & ONCE THROUGH \\
\hline $\begin{array}{l}\text { GRAND } \\
\text { TOWER }\end{array}$ & $\begin{array}{l}\text { CENT ILLINOIS } \\
\text { PUBLIC SERV }\end{array}$ & IL & 3253 & 862 & 4 & 114 & Operational & 1958 & COAL & FRESH & ONCE THROUGH \\
\hline GREEN RIVER & $\begin{array}{l}\text { KENTUCKY } \\
\text { UTILITIES CO }\end{array}$ & KY & 10171 & 1357 & 2 & 37.5 & Operational & 1950 & COAL & FRESH & ONCE THROUGH \\
\hline GREEN RIVER & $\begin{array}{l}\text { KENTUCKY } \\
\text { UTILITIES CO }\end{array}$ & KY & 10171 & 1357 & 3 & 75 & Operational & 1954 & COAL & FRESH & ONCE THROUGH \\
\hline GREEN RIVER & $\begin{array}{l}\text { KENTUCKY } \\
\text { UTILITIES CO }\end{array}$ & KY & 10171 & 1357 & 4 & 114 & Operational & 1959 & COAL & FRESH & ONCE THROUGH \\
\hline GREEN RIVER & $\begin{array}{l}\text { KENTUCKY } \\
\text { UTILITIES CO }\end{array}$ & KY & 10171 & 1357 & 1 & 37.5 & Operational & 1950 & COAL & FRESH & ONCE THROUGH \\
\hline $\begin{array}{l}\text { GREENE } \\
\text { COUNTY (AL) }\end{array}$ & $\begin{array}{l}\text { ALABAMA POWER } \\
\text { CO }\end{array}$ & $\mathrm{AL}$ & 195 & 10 & 1 & 299 & Operational & 1965 & COAL & FRESH & ONCE THROUGH \\
\hline $\begin{array}{l}\text { GREENE } \\
\text { COUNTY (AL) }\end{array}$ & $\begin{array}{l}\text { ALABAMA POWER } \\
\text { CO }\end{array}$ & $\mathrm{AL}$ & 195 & 10 & 2 & 269 & Operational & 1966 & COAL & FRESH & ONCE THROUGH \\
\hline GREENIDGE & $\begin{array}{l}\text { NEW YORK STATE } \\
\text { ELEC \& GAS }\end{array}$ & NY & 13511 & 2527 & 3 & 58.8 & Operational & 1950 & COAL & FRESH & ONCE THROUGH \\
\hline GREENIDGE & $\begin{array}{l}\text { NEW YORK STATE } \\
\text { ELEC \& GAS }\end{array}$ & NY & 13511 & 2527 & 4 & 113 & Operational & 1953 & COAL & FRESH & ONCE THROUGH \\
\hline GREENVILLE & $\begin{array}{l}\text { GREENVILLE ELEC } \\
\text { DEPT }\end{array}$ & TX & 7634 & 4195 & 1 & 22.1 & Operational & 1966 & GAS & FRESH & ONCE THROUGH \\
\hline GREENVILLE & $\begin{array}{l}\text { GREENVILLE ELEC } \\
\text { DEPT }\end{array}$ & TX & 7634 & 4195 & 2 & 29.4 & Operational & 1969 & GAS & FRESH & ONCE THROUGH \\
\hline GREENVILLE & $\begin{array}{l}\text { GREENVILLE ELEC } \\
\text { DEPT }\end{array}$ & TX & 7634 & 4195 & 3 & 48 & Operational & 1977 & GAS & FRESH & ONCE THROUGH \\
\hline HA WAGNER & $\begin{array}{l}\text { BALTIMORE GAS \& } \\
\text { ELEC CO }\end{array}$ & MD & 1167 & 1554 & 1 & 133 & Operational & 1956 & OIL & BRACKISH & ONCE THROUGH \\
\hline
\end{tabular}




\begin{tabular}{|c|c|c|c|c|c|c|c|c|c|c|c|}
\hline Plant Name & Utility Name & State & Utility/P & $n t / U n i$ & $I D$ & $M W$ & Status & Year & Fuel & Water & Cooling Systen \\
\hline HA WAGNER & $\begin{array}{l}\text { BALTIMORE GAS \& } \\
\text { ELEC CO }\end{array}$ & MD & 1167 & 1554 & 2 & 136 & Operational & 1959 & COAL & BRACKISH & ONCE THROUGH \\
\hline HA WAGNER & $\begin{array}{l}\text { BALTIMORE GAS \& } \\
\text { ELEC CO }\end{array}$ & MD & 1167 & 1554 & 3 & 359 & Operational & 1966 & COAL & BRACKISH & ONCE THROUGH \\
\hline HA WAGNER & $\begin{array}{l}\text { BALTIMORE GAS \& } \\
\text { ELEC CO }\end{array}$ & MD & 1167 & 1554 & 4 & 415 & Operational & 1972 & OIL & BRACKISH & ONCE THROUGH \\
\hline $\begin{array}{l}\text { HAMILTON } \\
(\mathrm{OH})\end{array}$ & $\begin{array}{l}\text { HAMILTON DEPT } \\
\text { PUBLIC UTIL }\end{array}$ & $\mathrm{OH}$ & 7977 & 2917 & 9 & 50.6 & Operational & 1975 & GAS & FRESH & ONCE THROUGH \\
\hline $\begin{array}{l}\text { HAMILTON } \\
(\mathrm{OH})\end{array}$ & $\begin{array}{l}\text { HAMILTON DEPT } \\
\text { PUBLIC UTIL }\end{array}$ & $\mathrm{OH}$ & 7977 & 2917 & 8 & 25 & Operational & 1965 & COAL & FRESH & ONCE THROUGH \\
\hline $\begin{array}{l}\text { HAMILTON } \\
(\mathrm{OH})\end{array}$ & $\begin{array}{l}\text { HAMILTON DEPT } \\
\text { PUBLIC UTIL }\end{array}$ & $\mathrm{OH}$ & 7977 & 2917 & 7 & 25 & Standby & 1960 & GAS & FRESH & ONCE THROUGH \\
\hline HAMMOND & $\begin{array}{l}\text { GEORGIA POWER } \\
\text { CO }\end{array}$ & GA & 7140 & 708 & 1 & 125 & Operational & 1954 & COAL & FRESH & ONCE THROUGH \\
\hline HAMMOND & $\begin{array}{l}\text { GEORGIA POWER } \\
\text { CO }\end{array}$ & GA & 7140 & 708 & 2 & 125 & Operational & 1954 & COAL & FRESH & ONCE THROUGH \\
\hline HAMMOND & $\begin{array}{l}\text { GEORGIA POWER } \\
\text { CO }\end{array}$ & GA & 7140 & 708 & 3 & 125 & Operational & 1955 & COAL & FRESH & ONCE THROUGH \\
\hline HAMMOND & $\begin{array}{l}\text { GEORGIA POWER } \\
\text { CO }\end{array}$ & GA & 7140 & 708 & 4 & 578 & Operational & 1970 & COAL & FRESH & ONCE THROUGH \\
\hline $\begin{array}{l}\text { HARBOR } \\
\text { BEACH }\end{array}$ & $\begin{array}{l}\text { DETROIT EDISON } \\
\text { CO }\end{array}$ & MI & 5109 & 1731 & 1 & 121 & Operational & 1968 & COAL & FRESH & ONCE THROUGH \\
\hline $\begin{array}{l}\text { HARLLEE } \\
\text { BRANCH }\end{array}$ & $\begin{array}{l}\text { GEORGIA POWER } \\
\text { CO }\end{array}$ & GA & 7140 & 709 & 1 & 299 & Operational & 1965 & COAL & FRESH & ONCE THROUGH \\
\hline $\begin{array}{l}\text { HARLLEE } \\
\text { BRANCH }\end{array}$ & $\begin{array}{l}\text { GEORGIA POWER } \\
\text { CO }\end{array}$ & GA & 7140 & 709 & 2 & 359 & Operational & 1967 & COAL & FRESH & ONCE THROUGH \\
\hline $\begin{array}{l}\text { HARLLEE } \\
\text { BRANCH }\end{array}$ & $\begin{array}{l}\text { GEORGIA POWER } \\
\text { CO }\end{array}$ & GA & 7140 & 709 & 3 & 544 & Operational & 1968 & COAL & FRESH & ONCE THROUGH \\
\hline
\end{tabular}




\begin{tabular}{|c|c|c|c|c|c|c|c|c|c|c|c|}
\hline Plant Name & Utility Name & State & Utility/Pl & $n t / U n i$ & $I D$ & $\boldsymbol{M W}$ & Status & Year & Fuel & Water & Cooling System \\
\hline $\begin{array}{l}\text { HARLLEE } \\
\text { BRANCH }\end{array}$ & $\begin{array}{l}\text { GEORGIA POWER } \\
\text { CO }\end{array}$ & GA & 7140 & 709 & 4 & 544 & Operational & 1969 & COAL & FRESH & ONCE THROUGH \\
\hline HAVANA & ILLINOIS POWER CO & $\mathrm{IL}$ & 9208 & 891 & 3 & 46 & Standby & 1948 & OIL & FRESH & ONCE THROUGH \\
\hline HAVANA & ILLINOIS POWER CO & $\mathrm{IL}$ & 9208 & 891 & 4 & 46 & Standby & 1950 & OIL & FRESH & ONCE THROUGH \\
\hline HAVANA & ILLINOIS POWER CO & IL & 9208 & 891 & 1 & 46 & Standby & 1947 & OIL & FRESH & ONCE THROUGH \\
\hline HAVANA & ILLINOIS POWER CO & $\mathrm{IL}$ & 9208 & 891 & 5 & 46 & Standby & 1950 & OIL & FRESH & ONCE THROUGH \\
\hline HAVANA & ILLINOIS POWER CO & $\mathrm{IL}$ & 9208 & 891 & 2 & 46 & Standby & 1947 & OIL & FRESH & ONCE THROUGH \\
\hline HAWTHORN & $\begin{array}{l}\text { KANSAS CITY } \\
\text { POWER \& LIGHT }\end{array}$ & MO & 10000 & 2079 & 5 & 515 & Operational & 1969 & COAL & FRESH & ONCE THROUGH \\
\hline HAYNES & $\begin{array}{l}\text { LOS ANGELES DEPT } \\
\text { WTR PWR }\end{array}$ & $\mathrm{CA}$ & 11208 & 400 & 3 & 230 & Operational & 1964 & GAS & SALINE & ONCE THROUGH \\
\hline HAYNES & $\begin{array}{l}\text { LOS ANGELES DEPT } \\
\text { WTR PWR }\end{array}$ & $\mathrm{CA}$ & 11208 & 400 & 6 & 343 & Operational & 1967 & GAS & SALINE & ONCE THROUGH \\
\hline HAYNES & $\begin{array}{l}\text { LOS ANGELES DEPT } \\
\text { WTR PWR }\end{array}$ & $\mathrm{CA}$ & 11208 & 400 & 4 & 230 & Operational & 1965 & GAS & SALINE & ONCE THROUGH \\
\hline HAYNES & $\begin{array}{l}\text { LOS ANGELES DEPT } \\
\text { WTR PWR }\end{array}$ & $\mathrm{CA}$ & 11208 & 400 & 2 & 230 & Operational & 1963 & GAS & SALINE & ONCE THROUGH \\
\hline HAYNES & $\begin{array}{l}\text { LOS ANGELES DEPT } \\
\text { WTR PWR }\end{array}$ & $\mathrm{CA}$ & 11208 & 400 & 1 & 230 & Operational & 1962 & GAS & SALINE & ONCE THROUGH \\
\hline HAYNES & $\begin{array}{l}\text { LOS ANGELES DEPT } \\
\text { WTR PWR }\end{array}$ & $\mathrm{CA}$ & 11208 & 400 & 5 & 343 & Operational & 1966 & GAS & SALINE & ONCE THROUGH \\
\hline HEALY & $\begin{array}{l}\text { GOLDEN VALLEY } \\
\text { ELEC ASSN }\end{array}$ & $\mathrm{AK}$ & 7353 & 6288 & 1 & 25 & Operational & 1967 & COAL & FRESH & ONCE THROUGH \\
\hline
\end{tabular}




\begin{tabular}{|c|c|c|c|c|c|c|c|c|c|c|c|}
\hline Plant Name & Utility Name & State & Utility/P & $n t / U n i$ & $I D$ & $M W$ & Status & Year & Fuel & Water & Cooling System \\
\hline $\begin{array}{l}\text { HENDERSON } \\
\text { (MS) }\end{array}$ & $\begin{array}{l}\text { GREENWOOD } \\
\text { UTILITIES }\end{array}$ & MS & 7651 & 2062 & 1 & 12.7 & Operational & 1956 & GAS & GROUND & ONCE THROUGH \\
\hline $\begin{array}{l}\text { HENDERSON } \\
\text { ONE }\end{array}$ & $\begin{array}{l}\text { HENDERSON MUN } \\
\text { POWER \& LT }\end{array}$ & KY & 8449 & 1372 & 6 & 32.3 & Operational & 1968 & COAL & FRESH & ONCE THROUGH \\
\hline $\begin{array}{l}\text { HENDERSON } \\
\text { ONE }\end{array}$ & $\begin{array}{l}\text { HENDERSON MUN } \\
\text { POWER \& LT }\end{array}$ & KY & 8449 & 1372 & 5 & 11.5 & Operational & 1957 & COAL & FRESH & ONCE THROUGH \\
\hline HENNEPIN & ILLINOIS POWER CO & IL & 9208 & 892 & 1 & 75 & Operational & 1953 & COAL & FRESH & ONCE THROUGH \\
\hline HENNEPIN & ILLINOIS POWER CO & $\mathrm{IL}$ & 9208 & 892 & 2 & 231 & Operational & 1959 & COAL & FRESH & ONCE THROUGH \\
\hline HESKETT & $\begin{array}{l}\text { MONTANA-DAKOTA } \\
\text { UTILITIES }\end{array}$ & ND & 12819 & 2790 & 1 & 40 & Operational & 1954 & COAL & FRESH & ONCE THROUGH \\
\hline HESKETT & $\begin{array}{l}\text { MONTANA-DAKOTA } \\
\text { UTILITIES }\end{array}$ & ND & 12819 & 2790 & 2 & 75 & Operational & 1963 & COAL & FRESH & ONCE THROUGH \\
\hline HICKLING & $\begin{array}{l}\text { NEW YORK STATE } \\
\text { ELEC \& GAS }\end{array}$ & NY & 13511 & 2529 & 1 & 37.5 & Operational & 1948 & COAL & FRESH & ONCE THROUGH \\
\hline HICKLING & $\begin{array}{l}\text { NEW YORK STATE } \\
\text { ELEC \& GAS }\end{array}$ & NY & 13511 & 2529 & 2 & 49 & Operational & 1952 & COAL & FRESH & ONCE THROUGH \\
\hline HIGH BRIDGE & $\begin{array}{l}\text { NORTHERN STATES } \\
\text { POWER CO }\end{array}$ & MN & 13781 & 1912 & 4 & 62.5 & Operational & 1944 & COAL & FRESH & ONCE THROUGH \\
\hline HIGH BRIDGE & $\begin{array}{l}\text { NORTHERN STATES } \\
\text { POWER CO }\end{array}$ & MN & 13781 & 1912 & 5 & 114 & Operational & 1956 & COAL & FRESH & ONCE THROUGH \\
\hline HIGH BRIDGE & $\begin{array}{l}\text { NORTHERN STATES } \\
\text { POWER CO }\end{array}$ & $\mathrm{MN}$ & 13781 & 1912 & 6 & 163 & Operational & 1959 & COAL & FRESH & ONCE THROUGH \\
\hline HOLLY STREET & $\begin{array}{l}\text { AUSTIN ELECTRIC } \\
\text { DEPT }\end{array}$ & $\mathrm{TX}$ & 1015 & 3549 & 4 & 210 & Operational & 1974 & GAS & FRESH & ONCE THROUGH \\
\hline HOLLY STREET & $\begin{array}{l}\text { AUSTIN ELECTRIC } \\
\text { DEPT }\end{array}$ & TX & 1015 & 3549 & 1 & 113 & Operational & 1960 & GAS & FRESH & ONCE THROUGH \\
\hline
\end{tabular}




\begin{tabular}{|c|c|c|c|c|c|c|c|c|c|c|c|}
\hline Plant Name & Utility Name & State & Utility/Pl & th/Uni & $I D$ & $M W$ & Status & Year & Fuel & Water & Cooling System \\
\hline HOLLY STREET & $\begin{array}{l}\text { AUSTIN ELECTRIC } \\
\text { DEPT }\end{array}$ & TX & 1015 & 3549 & 2 & 113 & Operational & 1964 & GAS & FRESH & ONCE THROUGH \\
\hline HOLLY STREET & $\begin{array}{l}\text { AUSTIN ELECTRIC } \\
\text { DEPT }\end{array}$ & TX & 1015 & 3549 & 3 & 190 & Operational & 1966 & GAS & FRESH & ONCE THROUGH \\
\hline HOLTWOOD & $\begin{array}{l}\text { PENNSYLVANIA } \\
\text { POWER \& LT }\end{array}$ & PA & 14715 & 3145 & 17 & 75 & Operational & 1954 & COAL & FRESH & ONCE THROUGH \\
\hline $\begin{array}{l}\text { HOOKERS } \\
\text { POINT }\end{array}$ & $\begin{array}{l}\text { TAMPA ELECTRIC } \\
\text { CO }\end{array}$ & $\mathrm{FL}$ & 18454 & 647 & 5 & 81.6 & Operational & 1955 & OIL & SALINE & ONCE THROUGH \\
\hline $\begin{array}{l}\text { HOOKERS } \\
\text { POINT }\end{array}$ & $\begin{array}{l}\text { TAMPA ELECTRIC } \\
\text { CO }\end{array}$ & $\mathrm{FL}$ & 18454 & 647 & 1 & 33 & Operational & 1948 & OIL & SALINE & ONCE THROUGH \\
\hline $\begin{array}{l}\text { HOOKERS } \\
\text { POINT }\end{array}$ & $\begin{array}{l}\text { TAMPA ELECTRIC } \\
\text { CO }\end{array}$ & $\mathrm{FL}$ & 18454 & 647 & 2 & 34.5 & Operational & 1950 & OIL & SALINE & ONCE THROUGH \\
\hline $\begin{array}{l}\text { HOOKERS } \\
\text { POINT }\end{array}$ & $\begin{array}{l}\text { TAMPA ELECTRIC } \\
\text { CO }\end{array}$ & $\mathrm{FL}$ & 18454 & 647 & 3 & 34.5 & Operational & 1950 & OIL & SALINE & ONCE THROUGH \\
\hline $\begin{array}{l}\text { HOOKERS } \\
\text { POINT }\end{array}$ & $\begin{array}{l}\text { TAMPA ELECTRIC } \\
\text { CO }\end{array}$ & FL & 18454 & 647 & 4 & 49 & Operational & 1953 & OIL & SALINE & ONCE THROUGH \\
\hline HOOT LAKE & $\begin{array}{l}\text { OTTER TAIL POWER } \\
\text { CO }\end{array}$ & MN & 14232 & 1943 & 3 & 75 & Operational & 1964 & COAL & FRESH & $\begin{array}{l}\text { COMBINATION (ONCE } \\
\text { THROUGH/HELPER TOWER) }\end{array}$ \\
\hline HOOT LAKE & $\begin{array}{l}\text { OTTER TAIL POWER } \\
\text { CO }\end{array}$ & MN & 14232 & 1943 & 2 & 54.4 & Operational & 1959 & COAL & FRESH & $\begin{array}{l}\text { COMBINATION (ONCE } \\
\text { THROUGH/HELPER TOWER) }\end{array}$ \\
\hline HOOT LAKE & $\begin{array}{l}\text { OTTER TAIL POWER } \\
\text { CO }\end{array}$ & MN & 14232 & 1943 & 1 & 7.5 & Operational & 1948 & COAL & FRESH & $\begin{array}{l}\text { COMBINATION (ONCE } \\
\text { THROUGH/HELPER TOWER) }\end{array}$ \\
\hline HT PRITCHARD & $\begin{array}{l}\text { INDIANAPOLIS } \\
\text { POWER \& LT }\end{array}$ & IN & 9273 & 991 & 6 & 114 & Operational & 1956 & COAL & FRESH & $\begin{array}{l}\text { COMBINATION (ONCE } \\
\text { THROUGH/HELPER TOWER) }\end{array}$ \\
\hline HT PRITCHARD & $\begin{array}{l}\text { INDIANAPOLIS } \\
\text { POWER \& LT }\end{array}$ & IN & 9273 & 991 & 5 & 69 & Operational & 1953 & COAL & FRESH & $\begin{array}{l}\text { COMBINATION (ONCE } \\
\text { THROUGH/HELPER TOWER) }\end{array}$ \\
\hline HT PRITCHARD & $\begin{array}{l}\text { INDIANAPOLIS } \\
\text { POWER \& LT }\end{array}$ & IN & 9273 & 991 & 4 & 69 & Operational & 1953 & COAL & FRESH & $\begin{array}{l}\text { COMBINATION (ONCE } \\
\text { THROUGH/HELPER TOWER) }\end{array}$ \\
\hline
\end{tabular}




\begin{tabular}{|c|c|c|c|c|c|c|c|c|c|c|c|}
\hline Plant Name & Utility Name & State & Utility/Pl & nt/Uni & $I D$ & $\boldsymbol{M W}$ & Status & Year & Fuel & Water & Cooling System \\
\hline HT PRITCHARD & $\begin{array}{l}\text { INDIANAPOLIS } \\
\text { POWER \& LT }\end{array}$ & IN & 9273 & 991 & 3 & 50 & Operational & 1951 & COAL & FRESH & $\begin{array}{l}\text { COMBINATION (ONCE } \\
\text { THROUGH/HELPER TOWER) }\end{array}$ \\
\hline HT PRITCHARD & $\begin{array}{l}\text { INDIANAPOLIS } \\
\text { POWER \& LT }\end{array}$ & IN & 9273 & 991 & 1 & 46 & Operational & 1949 & OIL & FRESH & $\begin{array}{l}\text { COMBINATION (ONCE } \\
\text { THROUGH/HELPER TOWER) }\end{array}$ \\
\hline HT PRITCHARD & $\begin{array}{l}\text { INDIANAPOLIS } \\
\text { POWER \& LT }\end{array}$ & IN & 9273 & 991 & 2 & 46 & Operational & 1950 & OIL & FRESH & $\begin{array}{l}\text { COMBINATION (ONCE } \\
\text { THROUGH/HELPER TOWER) }\end{array}$ \\
\hline HUDSON & $\begin{array}{l}\text { PUBLIC SERVICE } \\
\text { ELEC \& GAS }\end{array}$ & NJ & 15477 & 2403 & 1 & 455 & Operational & 1964 & GAS & BRACKISH & ONCE THROUGH \\
\hline HUDSON & $\begin{array}{l}\text { PUBLIC SERVICE } \\
\text { ELEC \& GAS }\end{array}$ & $\mathrm{NJ}$ & 15477 & 2403 & 2 & 660 & Operational & 1968 & COAL & BRACKISH & ONCE THROUGH \\
\hline HUMBOLDT & $\begin{array}{l}\text { CORN BELT POWER } \\
\text { COOP }\end{array}$ & IA & 4363 & 1213 & 1 & 9.4 & Standby & 1950 & COAL & FRESH & ONCE THROUGH \\
\hline HUMBOLDT & $\begin{array}{l}\text { CORN BELT POWER } \\
\text { COOP }\end{array}$ & IA & 4363 & 1213 & 2 & 9.4 & Standby & 1950 & COAL & FRESH & ONCE THROUGH \\
\hline HUMBOLDT & $\begin{array}{l}\text { CORN BELT POWER } \\
\text { COOP }\end{array}$ & IA & 4363 & 1213 & 3 & 13.5 & Operational & 1951 & COAL & FRESH & ONCE THROUGH \\
\hline HUMBOLDT & $\begin{array}{l}\text { CORN BELT POWER } \\
\text { COOP }\end{array}$ & IA & 4363 & 1213 & 4 & 20.3 & Operational & 1953 & COAL & FRESH & ONCE THROUGH \\
\hline $\begin{array}{l}\text { HUMBOLDT } \\
\text { BAY }\end{array}$ & $\begin{array}{l}\text { PACIFIC GAS \& } \\
\text { ELEC CO }\end{array}$ & $\mathrm{CA}$ & 14328 & 246 & 1 & 51.2 & Operational & 1956 & GAS & SALINE & ONCE THROUGH \\
\hline $\begin{array}{l}\text { HUMBOLDT } \\
\text { BAY }\end{array}$ & $\begin{array}{l}\text { PACIFIC GAS \& } \\
\text { ELEC CO }\end{array}$ & $\mathrm{CA}$ & 14328 & 246 & 2 & 51.2 & Operational & 1958 & GAS & SALINE & ONCE THROUGH \\
\hline HUNLOCK & UGI UTILITIES INC & PA & 19390 & 3176 & 3 & 50 & Operational & 1959 & COAL & FRESH & ONCE THROUGH \\
\hline $\begin{array}{l}\text { HUNTERS } \\
\text { POINT }\end{array}$ & $\begin{array}{l}\text { PACIFIC GAS \& } \\
\text { ELEC CO }\end{array}$ & $\mathrm{CA}$ & 14328 & 247 & 2 & 100 & Operational & 1948 & GAS & SALINE & ONCE THROUGH \\
\hline $\begin{array}{l}\text { HUNTERS } \\
\text { POINT }\end{array}$ & $\begin{array}{l}\text { PACIFIC GAS \& } \\
\text { ELEC CO }\end{array}$ & $\mathrm{CA}$ & 14328 & 247 & 3 & 100 & Operational & 1949 & GAS & SALINE & ONCE THROUGH \\
\hline
\end{tabular}




\begin{tabular}{|c|c|c|c|c|c|c|c|c|c|c|c|}
\hline Plant Name & Utility Name & State & Utility/Pl & it/Uni & $I D$ & $M W$ & Status & Year & Fuel & Water & Cooling System \\
\hline $\begin{array}{l}\text { HUNTERS } \\
\text { POINT }\end{array}$ & $\begin{array}{l}\text { PACIFIC GAS \& } \\
\text { ELEC CO }\end{array}$ & $\mathrm{CA}$ & 14328 & 247 & 4 & 156 & Operational & 1958 & GAS & SALINE & ONCE THROUGH \\
\hline $\begin{array}{l}\text { HUNTINGTON } \\
\text { BEACH }\end{array}$ & $\begin{array}{l}\text { SOUTHERN CALIF } \\
\text { EDISON CO }\end{array}$ & $\mathrm{CA}$ & 17609 & 335 & 1 & 218 & Operational & 1958 & GAS & SALINE & ONCE THROUGH \\
\hline $\begin{array}{l}\text { HUNTINGTON } \\
\text { BEACH }\end{array}$ & $\begin{array}{l}\text { SOUTHERN CALIF } \\
\text { EDISON CO }\end{array}$ & $\mathrm{CA}$ & 17609 & 335 & 4 & 218 & Operational & 1961 & GAS & SALINE & ONCE THROUGH \\
\hline $\begin{array}{l}\text { HUNTINGTON } \\
\text { BEACH }\end{array}$ & $\begin{array}{l}\text { SOUTHERN CALIF } \\
\text { EDISON CO }\end{array}$ & $\mathrm{CA}$ & 17609 & 335 & 3 & 218 & Operational & 1961 & GAS & SALINE & ONCE THROUGH \\
\hline $\begin{array}{l}\text { HUNTINGTON } \\
\text { BEACH }\end{array}$ & $\begin{array}{l}\text { SOUTHERN CALIF } \\
\text { EDISON CO }\end{array}$ & $\mathrm{CA}$ & 17609 & 335 & 2 & 218 & Operational & 1958 & GAS & SALINE & ONCE THROUGH \\
\hline HUTCHINGS & $\begin{array}{l}\text { DAYTON POWER \& } \\
\text { LIGHT CO }\end{array}$ & $\mathrm{OH}$ & 4922 & 2848 & 6 & 69 & Operational & 1953 & COAL & FRESH & ONCE THROUGH \\
\hline HUTCHINGS & $\begin{array}{l}\text { DAYTON POWER \& } \\
\text { LIGHT CO }\end{array}$ & $\mathrm{OH}$ & 4922 & 2848 & 1 & 69 & Operational & 1948 & COAL & FRESH & ONCE THROUGH \\
\hline HUTCHINGS & $\begin{array}{l}\text { DAYTON POWER \& } \\
\text { LIGHT CO }\end{array}$ & $\mathrm{OH}$ & 4922 & 2848 & 2 & 69 & Operational & 1949 & COAL & FRESH & ONCE THROUGH \\
\hline HUTCHINGS & $\begin{array}{l}\text { DAYTON POWER \& } \\
\text { LIGHT CO }\end{array}$ & $\mathrm{OH}$ & 4922 & 2848 & 3 & 69 & Operational & 1950 & COAL & FRESH & ONCE THROUGH \\
\hline HUTCHINGS & $\begin{array}{l}\text { DAYTON POWER \& } \\
\text { LIGHT CO }\end{array}$ & $\mathrm{OH}$ & 4922 & 2848 & 4 & 69 & Operational & 1951 & COAL & FRESH & ONCE THROUGH \\
\hline HUTCHINGS & $\begin{array}{l}\text { DAYTON POWER \& } \\
\text { LIGHT CO }\end{array}$ & $\mathrm{OH}$ & 4922 & 2848 & 5 & 69 & Operational & 1952 & COAL & FRESH & ONCE THROUGH \\
\hline HUTSONVILLE & $\begin{array}{l}\text { CENT ILLINOIS } \\
\text { PUBLIC SERV }\end{array}$ & IL & 3253 & 863 & 3 & 75 & Operational & 1953 & COAL & FRESH & ONCE THROUGH \\
\hline HUTSONVILLE & $\begin{array}{l}\text { CENT ILLINOIS } \\
\text { PUBLIC SERV }\end{array}$ & IL & 3253 & 863 & 4 & 75 & Operational & 1954 & COAL & FRESH & ONCE THROUGH \\
\hline IATAN & $\begin{array}{l}\text { KANSAS CITY } \\
\text { POWER \& LIGHT }\end{array}$ & MO & 10000 & 6065 & 1 & 726 & Operational & 1980 & COAL & FRESH & ONCE THROUGH \\
\hline
\end{tabular}




\begin{tabular}{|c|c|c|c|c|c|c|c|c|c|c|c|}
\hline Plant Name & Utility Name & State & Utility/Pl & nt/Uni & $I D$ & $M W$ & Status & Year & Fuel & Water & Cooling System \\
\hline $\begin{array}{l}\text { INDIAN POINT } \\
\text { TWO } 1\end{array}$ & $\begin{array}{l}\text { CONSOLIDATED } \\
\text { EDISON CO }\end{array}$ & NY & 4226 & 2497 & 1 & 1013 & Operational & 1974 & UR & BRACKISH & ONCE THROUGH \\
\hline $\begin{array}{l}\text { INDIAN POINT } \\
\text { THREE } 1\end{array}$ & $\begin{array}{l}\text { NEW YORK POWER } \\
\text { AUTHORITY }\end{array}$ & NY & 15296 & 8907 & 1 & 1013 & Operational & 1976 & UR & BRACKISH & ONCE THROUGH \\
\hline $\begin{array}{l}\text { INDIAN RIVER } \\
\text { (DE) }\end{array}$ & $\begin{array}{l}\text { DELMARVA POWER } \\
\& \text { LIGHT CO }\end{array}$ & DE & 5027 & 594 & 1 & 81.6 & Operational & 1957 & COAL & BRACKISH & ONCE THROUGH \\
\hline $\begin{array}{l}\text { INDIAN RIVER } \\
\text { (DE) }\end{array}$ & $\begin{array}{l}\text { DELMARVA POWER } \\
\& \text { LIGHT CO }\end{array}$ & DE & 5027 & 594 & 2 & 81.6 & Operational & 1959 & COAL & BRACKISH & ONCE THROUGH \\
\hline $\begin{array}{l}\text { INDIAN RIVER } \\
\text { (DE) }\end{array}$ & $\begin{array}{l}\text { DELMARVA POWER } \\
\text { \& LIGHT CO }\end{array}$ & DE & 5027 & 594 & 3 & 177 & Operational & 1970 & COAL & BRACKISH & ONCE THROUGH \\
\hline $\begin{array}{l}\text { INDIAN RIVER } \\
(\mathrm{FL})\end{array}$ & $\begin{array}{l}\text { ORLANDO UTILITIES } \\
\text { COMM }\end{array}$ & $\mathrm{FL}$ & 14610 & 683 & 1 & 86.7 & Operational & 1960 & GAS & BRACKISH & ONCE THROUGH \\
\hline $\begin{array}{l}\text { INDIAN RIVER } \\
(\mathrm{FL})\end{array}$ & $\begin{array}{l}\text { ORLANDO UTILITIES } \\
\text { COMM }\end{array}$ & $\mathrm{FL}$ & 14610 & 683 & 2 & 208 & Operational & 1964 & GAS & BRACKISH & ONCE THROUGH \\
\hline $\begin{array}{l}\text { INDIAN RIVER } \\
\text { (FL) }\end{array}$ & $\begin{array}{l}\text { ORLANDO UTILITIES } \\
\text { COMM }\end{array}$ & $\mathrm{FL}$ & 14610 & 683 & 3 & 345 & Operational & 1974 & GAS & BRACKISH & ONCE THROUGH \\
\hline IOLA & IOLA ELEC DEPT & KS & 9418 & 1291 & 5 & 5 & Standby & 1957 & GAS & FRESH & ONCE THROUGH \\
\hline IOLA & IOLA ELEC DEPT & KS & 9418 & 1291 & 4 & 3.5 & Operational & 1949 & GAS & FRESH & ONCE THROUGH \\
\hline JACK WATSON & $\begin{array}{l}\text { MISSISSIPPI } \\
\text { POWER CO }\end{array}$ & MS & 12686 & 2049 & 2 & 75 & Operational & 1960 & GAS & BRACKISH & $\begin{array}{l}\text { COMBINATION (ONCE } \\
\text { THROUGH/SPRAY CANAL) }\end{array}$ \\
\hline JACK WATSON & $\begin{array}{l}\text { MISSISSIPPI } \\
\text { POWER CO }\end{array}$ & MS & 12686 & 2049 & 1 & 75 & Operational & 1957 & GAS & BRACKISH & $\begin{array}{l}\text { COMBINATION (ONCE } \\
\text { THROUGH/SPRAY CANAL) }\end{array}$ \\
\hline JACK WATSON & $\begin{array}{l}\text { MISSISSIPPI } \\
\text { POWER CO }\end{array}$ & MS & 12686 & 2049 & 3 & 112 & Operational & 1962 & GAS & BRACKISH & $\begin{array}{l}\text { COMBINATION (ONCE } \\
\text { THROUGH/SPRAY CANAL) }\end{array}$ \\
\hline JACK WATSON & $\begin{array}{l}\text { MISSISSIPPI } \\
\text { POWER CO }\end{array}$ & MS & 12686 & 2049 & 4 & 250 & Operational & 1968 & GAS & BRACKISH & $\begin{array}{l}\text { COMBINATION (ONCE } \\
\text { THROUGH/SPRAY CANAL) }\end{array}$ \\
\hline
\end{tabular}




\begin{tabular}{|c|c|c|c|c|c|c|c|c|c|c|c|}
\hline Plant Name & Utility Name & State & Utility/Pl & $t /$ Uni & $I D$ & $M W$ & Status & Year & Fuel & Water & Cooling System \\
\hline $\begin{array}{l}\text { JAMES } \\
\text { DEYOUNG }\end{array}$ & $\begin{array}{l}\text { HOLLAND BD } \\
\text { PUBLIC WORKS }\end{array}$ & MI & 8723 & 1830 & 3 & 11.5 & Operational & 1952 & COAL & FRESH & ONCE THROUGH \\
\hline $\begin{array}{l}\text { JAMES } \\
\text { DEYOUNG }\end{array}$ & $\begin{array}{l}\text { HOLLAND BD } \\
\text { PUBLIC WORKS }\end{array}$ & MI & 8723 & 1830 & 4 & 22 & Operational & 1962 & COAL & FRESH & ONCE THROUGH \\
\hline $\begin{array}{l}\text { JAMES } \\
\text { DEYOUNG }\end{array}$ & $\begin{array}{l}\text { HOLLAND BD } \\
\text { PUBLIC WORKS }\end{array}$ & MI & 8723 & 1830 & 5 & 28.8 & Operational & 1969 & COAL & FRESH & ONCE THROUGH \\
\hline JAMES RIVER & $\begin{array}{l}\text { SPRINGFIELD } \\
\text { UTILITIES }\end{array}$ & MO & 17833 & 2161 & 3 & 44 & Operational & 1960 & COAL & FRESH & $\begin{array}{l}\text { COMBINATION (ONCE } \\
\text { THROUGH/HELPER TOWER) }\end{array}$ \\
\hline JAMES RIVER & $\begin{array}{l}\text { SPRINGFIELD } \\
\text { UTILITIES }\end{array}$ & MO & 17833 & 2161 & 4 & 60 & Operational & 1964 & COAL & FRESH & $\begin{array}{l}\text { COMBINATION (ONCE } \\
\text { THROUGH/HELPER TOWER) }\end{array}$ \\
\hline JAMES RIVER & $\begin{array}{l}\text { SPRINGFIELD } \\
\text { UTILITIES }\end{array}$ & MO & 17833 & 2161 & 2 & 22 & Operational & 1957 & COAL & FRESH & $\begin{array}{l}\text { COMBINATION (ONCE } \\
\text { THROUGH/HELPER TOWER) }\end{array}$ \\
\hline JAMES RIVER & $\begin{array}{l}\text { SPRINGFIELD } \\
\text { UTILITIES }\end{array}$ & MO & 17833 & 2161 & 1 & 22 & Operational & 1957 & COAL & FRESH & $\begin{array}{l}\text { COMBINATION (ONCE } \\
\text { THROUGH/HELPER TOWER) }\end{array}$ \\
\hline JAMES RIVER & $\begin{array}{l}\text { SPRINGFIELD } \\
\text { UTILITIES }\end{array}$ & MO & 17833 & 2161 & 5 & 105 & Operational & 1970 & COAL & FRESH & $\begin{array}{l}\text { COMBINATION (ONCE } \\
\text { THROUGH/HELPER TOWER) }\end{array}$ \\
\hline JB SIMS & $\begin{array}{l}\text { GRAND HAVEN BD } \\
\text { LT \& PWR }\end{array}$ & MI & 7483 & 1825 & 1 & 7.5 & Standby & 1961 & COAL & FRESH & $\begin{array}{l}\text { COMBINATION (ONCE } \\
\text { THROUGH/HELPER POND) }\end{array}$ \\
\hline JB SIMS & $\begin{array}{l}\text { GRAND HAVEN BD } \\
\text { LT \& PWR }\end{array}$ & MI & 7483 & 1825 & 2 & 7.5 & Standby & 1961 & COAL & FRESH & $\begin{array}{l}\text { COMBINATION (ONCE } \\
\text { THROUGH/HELPER POND) }\end{array}$ \\
\hline JB SIMS & $\begin{array}{l}\text { GRAND HAVEN BD } \\
\text { LT \& PWR }\end{array}$ & MI & 7483 & 1825 & 3 & 65 & Operational & 1983 & COAL & GROUND & $\begin{array}{l}\text { COMBINATION (ONCE } \\
\text { THROUGH/HELPER POND) }\end{array}$ \\
\hline JC MCNEIL & $\begin{array}{l}\text { BURLINGTON } \\
\text { ELECTRIC DEPT }\end{array}$ & VT & 2548 & 589 & 1 & 50 & Operational & 1984 & WOOD & GROUND & ONCE THROUGH \\
\hline JC WEADOCK & $\begin{array}{l}\text { CONSUMERS } \\
\text { POWER CO }\end{array}$ & MI & 4254 & 1720 & 8 & 156 & Operational & 1958 & COAL & FRESH & ONCE THROUGH \\
\hline JC WEADOCK & $\begin{array}{l}\text { CONSUMERS } \\
\text { POWER CO }\end{array}$ & MI & 4254 & 1720 & 7 & 156 & Operational & 1955 & COAL & FRESH & ONCE THROUGH \\
\hline
\end{tabular}




\begin{tabular}{|c|c|c|c|c|c|c|c|c|c|c|c|}
\hline Plant Name & Utility Name & State & Utility/Pl & $n t / U n$ & $I D$ & $M W$ & Status & Year & Fuel & Water & Cooling System \\
\hline JD KENNEDY & $\begin{array}{l}\text { JACKSONVILLE } \\
\text { ELEC AUTH }\end{array}$ & $\mathrm{FL}$ & 9617 & 666 & 9 & 50 & Standby & 1958 & OIL & BRACKISH & ONCE THROUGH \\
\hline JD KENNEDY & $\begin{array}{l}\text { JACKSONVILLE } \\
\text { ELEC AUTH }\end{array}$ & $\mathrm{FL}$ & 9617 & 666 & 8 & 50 & Standby & 1955 & OIL & BRACKISH & ONCE THROUGH \\
\hline JD KENNEDY & $\begin{array}{l}\text { JACKSONVILLE } \\
\text { ELEC AUTH }\end{array}$ & $\mathrm{FL}$ & 9617 & 666 & 10 & 150 & Operational & 1961 & GAS & BRACKISH & ONCE THROUGH \\
\hline JE CORETTE & $\begin{array}{l}\text { MONTANA POWER } \\
\text { CO }\end{array}$ & MT & 12825 & 2187 & 1 & 191 & Operational & 1968 & COAL & FRESH & ONCE THROUGH \\
\hline JEFFERIES & $\begin{array}{l}\text { SOUTH CAROLINA } \\
\text { PUB SERV }\end{array}$ & SC & 17543 & 3319 & 1 & 50 & Standby & 1954 & OIL & FRESH & ONCE THROUGH \\
\hline JEFFERIES & $\begin{array}{l}\text { SOUTH CAROLINA } \\
\text { PUB SERV }\end{array}$ & SC & 17543 & 3319 & 2 & 50 & Standby & 1954 & OIL & FRESH & ONCE THROUGH \\
\hline JENNISON & $\begin{array}{l}\text { NEW YORK STATE } \\
\text { ELEC \& GAS }\end{array}$ & NY & 13511 & 2531 & 1 & 37.5 & Operational & 1945 & COAL & FRESH & ONCE THROUGH \\
\hline JENNISON & $\begin{array}{l}\text { NEW YORK STATE } \\
\text { ELEC \& GAS }\end{array}$ & NY & 13511 & 2531 & 2 & 37.5 & Operational & 1950 & COAL & FRESH & ONCE THROUGH \\
\hline JH CAMPBELL & $\begin{array}{l}\text { CONSUMERS } \\
\text { POWER CO }\end{array}$ & MI & 4254 & 1710 & 3 & 871 & Operational & 1980 & COAL & FRESH & ONCE THROUGH \\
\hline JH CAMPBELL & $\begin{array}{l}\text { CONSUMERS } \\
\text { POWER CO }\end{array}$ & MI & 4254 & 1710 & 1 & 265 & Operational & 1962 & COAL & FRESH & ONCE THROUGH \\
\hline JH CAMPBELL & $\begin{array}{l}\text { CONSUMERS } \\
\text { POWER CO }\end{array}$ & MI & 4254 & 1710 & 2 & 385 & Operational & 1967 & COAL & FRESH & ONCE THROUGH \\
\hline JM STUART & $\begin{array}{l}\text { DAYTON POWER \& } \\
\text { LIGHT CO }\end{array}$ & $\mathrm{OH}$ & 4922 & 2850 & 1 & 610 & Operational & 1971 & COAL & FRESH & ONCE THROUGH \\
\hline JM STUART & $\begin{array}{l}\text { DAYTON POWER \& } \\
\text { LIGHT CO }\end{array}$ & $\mathrm{OH}$ & 4922 & 2850 & 2 & 610 & Operational & 1971 & COAL & FRESH & ONCE THROUGH \\
\hline JM STUART & $\begin{array}{l}\text { DAYTON POWER \& } \\
\text { LIGHT CO }\end{array}$ & $\mathrm{OH}$ & 4922 & 2850 & 3 & 610 & Operational & 1972 & COAL & FRESH & ONCE THROUGH \\
\hline
\end{tabular}




\begin{tabular}{|c|c|c|c|c|c|c|c|c|c|c|c|}
\hline Plant Name & Utility Name & State & Utility/Pl & $n t / U n i$ & $I D$ & $M W$ & Status & Year & Fuel & Water & Cooling System \\
\hline JOHN SEVIER & $\begin{array}{l}\text { TENNESSEE } \\
\text { VALLEY AUTH }\end{array}$ & $\mathrm{TN}$ & 18642 & 3405 & 1 & 200 & Operational & 1955 & COAL & FRESH & ONCE THROUGH \\
\hline JOHN SEVIER & $\begin{array}{l}\text { TENNESSEE } \\
\text { VALLEY AUTH }\end{array}$ & TN & 18642 & 3405 & 2 & 200 & Operational & 1955 & COAL & FRESH & ONCE THROUGH \\
\hline JOHN SEVIER & $\begin{array}{l}\text { TENNESSEE } \\
\text { VALLEY AUTH }\end{array}$ & $\mathrm{TN}$ & 18642 & 3405 & 3 & 200 & Operational & 1956 & COAL & FRESH & ONCE THROUGH \\
\hline JOHN SEVIER & $\begin{array}{l}\text { TENNESSEE } \\
\text { VALLEY AUTH }\end{array}$ & $\mathrm{TN}$ & 18642 & 3405 & 4 & 200 & Operational & 1957 & COAL & FRESH & ONCE THROUGH \\
\hline $\begin{array}{l}\text { JOHNSONVILL } \\
\text { E (TN) }\end{array}$ & $\begin{array}{l}\text { TENNESSEE } \\
\text { VALLEY AUTH }\end{array}$ & TN & 18642 & 3406 & 6 & 147 & Operational & 1953 & COAL & FRESH & ONCE THROUGH \\
\hline $\begin{array}{l}\text { JOHNSONVILL } \\
\text { E (TN) }\end{array}$ & $\begin{array}{l}\text { TENNESSEE } \\
\text { VALLEY AUTH }\end{array}$ & TN & 18642 & 3406 & 9 & 173 & Operational & 1959 & COAL & FRESH & ONCE THROUGH \\
\hline $\begin{array}{l}\text { JOHNSONVILL } \\
\text { E (TN) }\end{array}$ & $\begin{array}{l}\text { TENNESSEE } \\
\text { VALLEY AUTH }\end{array}$ & TN & 18642 & 3406 & 7 & 173 & Operational & 1958 & COAL & FRESH & ONCE THROUGH \\
\hline $\begin{array}{l}\text { JOHNSONVILL } \\
\text { E (TN) }\end{array}$ & $\begin{array}{l}\text { TENNESSEE } \\
\text { VALLEY AUTH }\end{array}$ & TN & 18642 & 3406 & 5 & 147 & Operational & 1952 & COAL & FRESH & ONCE THROUGH \\
\hline $\begin{array}{l}\text { JOHNSONVILL } \\
\text { E (TN) }\end{array}$ & $\begin{array}{l}\text { TENNESSEE } \\
\text { VALLEY AUTH }\end{array}$ & TN & 18642 & 3406 & 3 & 125 & Operational & 1952 & COAL & FRESH & ONCE THROUGH \\
\hline $\begin{array}{l}\text { JOHNSONVILL } \\
\text { E (TN) }\end{array}$ & $\begin{array}{l}\text { TENNESSEE } \\
\text { VALLEY AUTH }\end{array}$ & TN & 18642 & 3406 & 8 & 173 & Operational & 1959 & COAL & FRESH & ONCE THROUGH \\
\hline $\begin{array}{l}\text { JOHNSONVILL } \\
\text { E (TN) }\end{array}$ & $\begin{array}{l}\text { TENNESSEE } \\
\text { VALLEY AUTH }\end{array}$ & TN & 18642 & 3406 & 2 & 125 & Operational & 1951 & COAL & FRESH & ONCE THROUGH \\
\hline $\begin{array}{l}\text { JOHNSONVILL } \\
\text { E (TN) }\end{array}$ & $\begin{array}{l}\text { TENNESSEE } \\
\text { VALLEY AUTH }\end{array}$ & TN & 18642 & 3406 & 10 & 173 & Operational & 1959 & COAL & FRESH & ONCE THROUGH \\
\hline $\begin{array}{l}\text { JOHNSONVILL } \\
\text { E (TN) }\end{array}$ & $\begin{array}{l}\text { TENNESSEE } \\
\text { VALLEY AUTH }\end{array}$ & TN & 18642 & 3406 & 1 & 125 & Operational & 1951 & COAL & FRESH & ONCE THROUGH \\
\hline $\begin{array}{l}\text { JOHNSONVILL } \\
\text { E (TN) }\end{array}$ & $\begin{array}{l}\text { TENNESSEE } \\
\text { VALLEY AUTH }\end{array}$ & TN & 18642 & 3406 & 4 & 125 & Operational & 1952 & COAL & FRESH & ONCE THROUGH \\
\hline
\end{tabular}




\begin{tabular}{|c|c|c|c|c|c|c|c|c|c|c|c|}
\hline Plant Name & Utility Name & State & Utility/Pl & $n t / U n i$ & $I D$ & $M W$ & Status & Year & Fuel & Water & Cooling System \\
\hline JOLIET & $\begin{array}{l}\text { COMMONWEALTH } \\
\text { EDISON CO }\end{array}$ & $\mathrm{IL}$ & 4110 & 874 & 6 & 360 & Operational & 1959 & COAL & FRESH & ONCE THROUGH \\
\hline JOLIET & $\begin{array}{l}\text { COMMONWEALTH } \\
\text { EDISON CO }\end{array}$ & $\mathrm{IL}$ & 4110 & 384 & 7 & 660 & Operational & 1965 & COAL & FRESH & ONCE THROUGH \\
\hline JOLIET & $\begin{array}{l}\text { COMMONWEALTH } \\
\text { EDISON CO }\end{array}$ & IL & 4110 & 384 & 8 & 660 & Operational & 1966 & COAL & FRESH & ONCE THROUGH \\
\hline JOPPA & $\begin{array}{l}\text { ELECTRIC ENERGY } \\
\text { INC }\end{array}$ & $\mathrm{IL}$ & 5748 & 887 & 2 & 183 & Operational & 1953 & COAL & FRESH & ONCE THROUGH \\
\hline JOPPA & $\begin{array}{l}\text { ELECTRIC ENERGY } \\
\text { INC }\end{array}$ & $\mathrm{IL}$ & 5748 & 887 & 3 & 183 & Operational & 1954 & COAL & FRESH & ONCE THROUGH \\
\hline JOPPA & $\begin{array}{l}\text { ELECTRIC ENERGY } \\
\text { INC }\end{array}$ & $\mathrm{IL}$ & 5748 & 887 & 4 & 183 & Operational & 1954 & COAL & FRESH & ONCE THROUGH \\
\hline JOPPA & $\begin{array}{l}\text { ELECTRIC ENERGY } \\
\text { INC }\end{array}$ & $\mathrm{IL}$ & 5748 & 887 & 5 & 183 & Operational & 1955 & COAL & FRESH & ONCE THROUGH \\
\hline JOPPA & $\begin{array}{l}\text { ELECTRIC ENERGY } \\
\text { INC }\end{array}$ & $\mathrm{IL}$ & 5748 & 887 & 6 & 183 & Operational & 1955 & COAL & FRESH & ONCE THROUGH \\
\hline JOPPA & $\begin{array}{l}\text { ELECTRIC ENERGY } \\
\text { INC }\end{array}$ & $\mathrm{IL}$ & 5748 & 887 & 1 & 183 & Operational & 1953 & COAL & FRESH & ONCE THROUGH \\
\hline JOSLIN & $\begin{array}{l}\text { CENTRAL POWER \& } \\
\text { LIGHT CO }\end{array}$ & $\mathrm{TX}$ & 3278 & 3436 & 1 & 261 & Operational & 1971 & GAS & BRACKISH & ONCE THROUGH \\
\hline JP PULLIAM & $\begin{array}{l}\text { WISCONSIN PUBLIC } \\
\text { SERVICE }\end{array}$ & WI & 20860 & 4072 & 4 & 30 & Operational & 1947 & COAL & FRESH & ONCE THROUGH \\
\hline JP PULLIAM & $\begin{array}{l}\text { WISCONSIN PUBLIC } \\
\text { SERVICE }\end{array}$ & WI & 20860 & 4072 & 8 & 125 & Operational & 1964 & COAL & FRESH & ONCE THROUGH \\
\hline JP PULLIAM & $\begin{array}{l}\text { WISCONSIN PUBLIC } \\
\text { SERVICE }\end{array}$ & WI & 20860 & 4072 & 7 & 75 & Operational & 1958 & COAL & FRESH & ONCE THROUGH \\
\hline JP PULLIAM & $\begin{array}{l}\text { WISCONSIN PUBLIC } \\
\text { SERVICE }\end{array}$ & WI & 20860 & 4072 & 5 & 50 & Operational & 1949 & COAL & FRESH & ONCE THROUGH \\
\hline
\end{tabular}




\begin{tabular}{|c|c|c|c|c|c|c|c|c|c|c|c|}
\hline Plant Name & Utility Name & State & Utility/Pl & $n t / U n i$ & $I D$ & $M W$ & Status & Year & Fuel & Water & Cooling System \\
\hline JP PULLIAM & $\begin{array}{l}\text { WISCONSIN PUBLIC } \\
\text { SERVICE }\end{array}$ & WI & 20860 & 4072 & 3 & 30 & Operational & 1943 & COAL & FRESH & ONCE THROUGH \\
\hline JP PULLIAM & $\begin{array}{l}\text { WISCONSIN PUBLIC } \\
\text { SERVICE }\end{array}$ & WI & 20860 & 4072 & 6 & 62.5 & Operational & 1951 & COAL & FRESH & ONCE THROUGH \\
\hline JR WHITING & $\begin{array}{l}\text { CONSUMERS } \\
\text { POWER CO }\end{array}$ & MI & 4254 & 1723 & 2 & 100 & Operational & 1952 & COAL & FRESH & ONCE THROUGH \\
\hline JR WHITING & $\begin{array}{l}\text { CONSUMERS } \\
\text { POWER CO }\end{array}$ & MI & 4254 & 1723 & 3 & 125 & Operational & 1953 & COAL & FRESH & ONCE THROUGH \\
\hline JR WHITING & $\begin{array}{l}\text { CONSUMERS } \\
\text { POWER CO }\end{array}$ & MI & 4254 & 1723 & 1 & 100 & Operational & 1952 & COAL & FRESH & ONCE THROUGH \\
\hline JS COOPER & $\begin{array}{l}\text { EAST KENTUCKY } \\
\text { POWER COOP }\end{array}$ & KY & 5580 & 1384 & 2 & 221 & Operational & 1969 & COAL & FRESH & ONCE THROUGH \\
\hline JS COOPER & $\begin{array}{l}\text { EAST KENTUCKY } \\
\text { POWER COOP }\end{array}$ & KY & 5580 & 1384 & 1 & 100 & Operational & 1965 & COAL & FRESH & ONCE THROUGH \\
\hline KAHE & $\begin{array}{l}\text { HAWAIIAN } \\
\text { ELECTRIC CO }\end{array}$ & $\mathrm{HI}$ & 19547 & 765 & 2 & 81.6 & Operational & 1964 & OIL & SALINE & ONCE THROUGH \\
\hline KAHE & $\begin{array}{l}\text { HAWAIIAN } \\
\text { ELECTRIC CO }\end{array}$ & $\mathrm{HI}$ & 19547 & 765 & 5 & 135 & Operational & 1974 & OIL & SALINE & ONCE THROUGH \\
\hline KAHE & $\begin{array}{l}\text { HAWAIIAN } \\
\text { ELECTRIC CO }\end{array}$ & $\mathrm{HI}$ & 19547 & 765 & 6 & 135 & Operational & 1981 & OIL & SALINE & ONCE THROUGH \\
\hline KAHE & $\begin{array}{l}\text { HAWAIIAN } \\
\text { ELECTRIC CO }\end{array}$ & $\mathrm{HI}$ & 19547 & 765 & 1 & 81.6 & Operational & 1963 & OIL & SALINE & ONCE THROUGH \\
\hline KAHE & $\begin{array}{l}\text { HAWAIIAN } \\
\text { ELECTRIC CO }\end{array}$ & $\mathrm{HI}$ & 19547 & 765 & 4 & 90.9 & Operational & 1972 & OIL & SALINE & ONCE THROUGH \\
\hline KAHE & $\begin{array}{l}\text { HAWAIIAN } \\
\text { ELECTRIC CO }\end{array}$ & $\mathrm{HI}$ & 19547 & 765 & 3 & 85.9 & Operational & 1970 & OIL & SALINE & ONCE THROUGH \\
\hline KAHULUI & MAUI ELECTRIC CO & $\mathrm{HI}$ & 11834 & 6056 & 1 & 5 & Operational & 1948 & OIL & GROUND & ONCE THROUGH \\
\hline
\end{tabular}




\begin{tabular}{|c|c|c|c|c|c|c|c|c|c|c|c|}
\hline Plant Name & Utility Name & State & Utility/Pl & $n t / U n$ & $I D$ & $M W$ & Status & Year & Fuel & Water & Cooling System \\
\hline KAHULUI & MAUI ELECTRIC CO & $\mathrm{HI}$ & 11834 & 6056 & 2 & 5 & Operational & 1949 & OIL & GROUND & ONCE THROUGH \\
\hline KAHULUI & MAUI ELECTRIC CO & $\mathrm{HI}$ & 11834 & 6056 & 3 & 11.5 & Operational & 1954 & OIL & GROUND & ONCE THROUGH \\
\hline KAHULUI & MAUI ELECTRIC CO & $\mathrm{HI}$ & 11834 & 6056 & 4 & 12.5 & Operational & 1966 & OIL & GROUND & ONCE THROUGH \\
\hline KAMMER & OHIO POWER CO & WV & 14006 & 3947 & 1 & 238 & Operational & 1958 & COAL & FRESH & ONCE THROUGH \\
\hline KAMMER & OHIO POWER CO & WV & 14006 & 3947 & 2 & 238 & Operational & 1958 & COAL & FRESH & ONCE THROUGH \\
\hline KAMMER & OHIO POWER CO & WV & 14006 & 3947 & 3 & 238 & Operational & 1959 & COAL & FRESH & ONCE THROUGH \\
\hline $\begin{array}{l}\text { KANAWHA } \\
\text { RIVER }\end{array}$ & $\begin{array}{l}\text { APPALACHIAN } \\
\text { POWER CO }\end{array}$ & WV & 733 & 3936 & 1 & 220 & Operational & 1953 & COAL & FRESH & ONCE THROUGH \\
\hline $\begin{array}{l}\text { KANAWHA } \\
\text { RIVER }\end{array}$ & $\begin{array}{l}\text { APPALACHIAN } \\
\text { POWER CO }\end{array}$ & WV & 733 & 3936 & 2 & 220 & Operational & 1953 & COAL & FRESH & ONCE THROUGH \\
\hline KAW (KS) & $\begin{array}{l}\text { KANSAS CITY BD } \\
\text { PUB UTIL }\end{array}$ & KS & 9996 & 1294 & 1 & 46 & Operational & 1955 & COAL & FRESH & ONCE THROUGH \\
\hline KAW (KS) & $\begin{array}{l}\text { KANSAS CITY BD } \\
\text { PUB UTIL }\end{array}$ & KS & 9996 & 1294 & 2 & 50 & Operational & 1957 & COAL & FRESH & ONCE THROUGH \\
\hline KAW (KS) & $\begin{array}{l}\text { KANSAS CITY BD } \\
\text { PUB UTIL }\end{array}$ & KS & 9996 & 1294 & 3 & 65.3 & Operational & 1962 & COAL & FRESH & ONCE THROUGH \\
\hline KEARNY (NJ) & $\begin{array}{l}\text { PUBLIC SERVICE } \\
\text { ELEC \& GAS }\end{array}$ & $\mathrm{NJ}$ & 15477 & 2404 & 7 & 157 & Operational & 1953 & OIL & BRACKISH & ONCE THROUGH \\
\hline KEARNY (NJ) & $\begin{array}{l}\text { PUBLIC SERVICE } \\
\text { ELEC \& GAS }\end{array}$ & $\mathrm{NJ}$ & 15477 & 2404 & 8 & 157 & Operational & 1953 & OIL & BRACKISH & ONCE THROUGH \\
\hline $\begin{array}{l}\text { KENDALL } \\
\text { SQUARE }\end{array}$ & $\begin{array}{l}\text { CAMBRIDGE ELEC } \\
\text { LIGHT CO }\end{array}$ & MA & 2886 & 1595 & 2 & 23 & Operational & 1951 & GAS & FRESH & ONCE THROUGH \\
\hline
\end{tabular}




\begin{tabular}{|c|c|c|c|c|c|c|c|c|c|c|c|}
\hline Plant Name & Utility Name & State & Utility/Pl & $n t / U n i$ & $I D$ & $\boldsymbol{M W}$ & Status & Year & Fuel & Water & Cooling System \\
\hline $\begin{array}{l}\text { KENDALL } \\
\text { SQUARE }\end{array}$ & $\begin{array}{l}\text { CAMBRIDGE ELEC } \\
\text { LIGHT CO }\end{array}$ & MA & 2886 & 1595 & 3 & 27.2 & Operational & 1958 & GAS & FRESH & ONCE THROUGH \\
\hline $\begin{array}{l}\text { KENDALL } \\
\text { SQUARE }\end{array}$ & $\begin{array}{l}\text { CAMBRIDGE ELEC } \\
\text { LIGHT CO }\end{array}$ & MA & 2886 & 1595 & 1 & 17.3 & Operational & 1949 & GAS & FRESH & ONCE THROUGH \\
\hline KEWAUNEE & $\begin{array}{l}\text { WISCONSIN PUBLIC } \\
\text { SERVICE }\end{array}$ & WI & 20860 & 8024 & 1 & 560 & Operational & 1974 & UR & FRESH & ONCE THROUGH \\
\hline KING & $\begin{array}{l}\text { FORT PIERCE UTIL } \\
\text { AUTH }\end{array}$ & $\mathrm{FL}$ & 6616 & 658 & 6 & 16.5 & Standby & 1958 & GAS & SALINE & ONCE THROUGH \\
\hline KING & $\begin{array}{l}\text { FORT PIERCE UTIL } \\
\text { AUTH }\end{array}$ & $\mathrm{FL}$ & 6616 & 658 & 7 & 33 & Standby & 1964 & GAS & SALINE & ONCE THROUGH \\
\hline KINGSTON & $\begin{array}{l}\text { TENNESSEE } \\
\text { VALLEY AUTH }\end{array}$ & $\mathrm{TN}$ & 18642 & 3407 & 4 & 175 & Operational & 1954 & COAL & FRESH & ONCE THROUGH \\
\hline KINGSTON & $\begin{array}{l}\text { TENNESSEE } \\
\text { VALLEY AUTH }\end{array}$ & $\mathrm{TN}$ & 18642 & 3407 & 9 & 200 & Operational & 1955 & COAL & FRESH & ONCE THROUGH \\
\hline KINGSTON & $\begin{array}{l}\text { TENNESSEE } \\
\text { VALLEY AUTH }\end{array}$ & $\mathrm{TN}$ & 18642 & 3407 & 8 & 200 & Operational & 1955 & COAL & FRESH & ONCE THROUGH \\
\hline KINGSTON & $\begin{array}{l}\text { TENNESSEE } \\
\text { VALLEY AUTH }\end{array}$ & $\mathrm{TN}$ & 18642 & 3407 & 7 & 200 & Operational & 1955 & COAL & FRESH & ONCE THROUGH \\
\hline KINGSTON & $\begin{array}{l}\text { TENNESSEE } \\
\text { VALLEY AUTH }\end{array}$ & $\mathrm{TN}$ & 18642 & 3407 & 6 & 200 & Operational & 1955 & COAL & FRESH & ONCE THROUGH \\
\hline KINGSTON & $\begin{array}{l}\text { TENNESSEE } \\
\text { VALLEY AUTH }\end{array}$ & $\mathrm{TN}$ & 18642 & 3407 & 3 & 175 & Operational & 1954 & COAL & FRESH & ONCE THROUGH \\
\hline KINGSTON & $\begin{array}{l}\text { TENNESSEE } \\
\text { VALLEY AUTH }\end{array}$ & $\mathrm{TN}$ & 18642 & 3407 & 2 & 175 & Operational & 1954 & COAL & FRESH & ONCE THROUGH \\
\hline KINGSTON & $\begin{array}{l}\text { TENNESSEE } \\
\text { VALLEY AUTH }\end{array}$ & $\mathrm{TN}$ & 18642 & 3407 & 1 & 175 & Operational & 1954 & COAL & FRESH & ONCE THROUGH \\
\hline KINGSTON & $\begin{array}{l}\text { TENNESSEE } \\
\text { VALLEY AUTH }\end{array}$ & $\mathrm{TN}$ & 18642 & 3407 & 5 & 200 & Operational & 1955 & COAL & FRESH & ONCE THROUGH \\
\hline
\end{tabular}




\begin{tabular}{|c|c|c|c|c|c|c|c|c|c|c|c|}
\hline Plant Name & Utility Name & State & Utility/P & $n t / U n i$ & $I D$ & $M W$ & Status & Year & Fuel & Water & Cooling System \\
\hline KINTIGH & $\begin{array}{l}\text { NEW YORK STATE } \\
\text { ELEC \& GAS }\end{array}$ & NY & 13511 & 6082 & 1 & 655 & Operational & 1984 & COAL & FRESH & ONCE THROUGH \\
\hline KRAFT & $\begin{array}{l}\text { SAVANNAH ELEC \& } \\
\text { POWER CO }\end{array}$ & GA & 16687 & 733 & 1 & 50 & Operational & 1958 & COAL & BRACKISH & ONCE THROUGH \\
\hline KRAFT & $\begin{array}{l}\text { SAVANNAH ELEC \& } \\
\text { POWER CO }\end{array}$ & GA & 16687 & 733 & 2 & 54.4 & Operational & 1961 & COAL & BRACKISH & ONCE THROUGH \\
\hline KRAFT & $\begin{array}{l}\text { SAVANNAH ELEC \& } \\
\text { POWER CO }\end{array}$ & GA & 16687 & 733 & 3 & 104 & Operational & 1965 & COAL & BRACKISH & ONCE THROUGH \\
\hline KRAFT & $\begin{array}{l}\text { SAVANNAH ELEC \& } \\
\text { POWER CO }\end{array}$ & GA & 16687 & 733 & 4 & 126 & Operational & 1972 & OIL & BRACKISH & ONCE THROUGH \\
\hline KYGER CREEK & $\begin{array}{l}\text { OHIO VALLEY ELEC } \\
\text { CORP }\end{array}$ & $\mathrm{OH}$ & 14015 & 2876 & 1 & 217 & Operational & 1955 & COAL & FRESH & ONCE THROUGH \\
\hline KYGER CREEK & $\begin{array}{l}\text { OHIO VALLEY ELEC } \\
\text { CORP }\end{array}$ & $\mathrm{OH}$ & 14015 & 2876 & 5 & 217 & Operational & 1955 & COAL & FRESH & ONCE THROUGH \\
\hline KYGER CREEK & $\begin{array}{l}\text { OHIO VALLEY ELEC } \\
\text { CORP }\end{array}$ & $\mathrm{OH}$ & 14015 & 2876 & 4 & 217 & Operational & 1955 & COAL & FRESH & ONCE THROUGH \\
\hline KYGER CREEK & $\begin{array}{l}\text { OHIO VALLEY ELEC } \\
\text { CORP }\end{array}$ & $\mathrm{OH}$ & 14015 & 2876 & 3 & 217 & Operational & 1955 & COAL & FRESH & ONCE THROUGH \\
\hline KYGER CREEK & $\begin{array}{l}\text { OHIO VALLEY ELEC } \\
\text { CORP }\end{array}$ & $\mathrm{OH}$ & 14015 & 2876 & 2 & 217 & Operational & 1955 & COAL & FRESH & ONCE THROUGH \\
\hline KYRENE & $\begin{array}{l}\text { SALT RIVER } \\
\text { PROJECT }\end{array}$ & $A Z$ & 16572 & 147 & 1 & 34.5 & Standby & 1952 & GAS & FRESH & $\begin{array}{l}\text { COMBINATION (ONCE } \\
\text { THROUGH/HELPER TOWER) }\end{array}$ \\
\hline KYRENE & $\begin{array}{l}\text { SALT RIVER } \\
\text { PROJECT }\end{array}$ & $A Z$ & 16572 & 147 & 2 & 73.5 & Standby & 1954 & GAS & FRESH & $\begin{array}{l}\text { COMBINATION (ONCE } \\
\text { THROUGH/HELPER TOWER) }\end{array}$ \\
\hline LABADIE & UNION ELECTRIC CO & $\mathrm{MO}$ & 19436 & 2103 & 1 & 621 & Operational & 1970 & COAL & FRESH & ONCE THROUGH \\
\hline LABADIE & UNION ELECTRIC CO & $\mathrm{MO}$ & 19436 & 2103 & 2 & 621 & Operational & 1971 & COAL & FRESH & ONCE THROUGH \\
\hline
\end{tabular}




\begin{tabular}{|c|c|c|c|c|c|c|c|c|c|c|c|}
\hline Plant Name & Utility Name & State & Utility/P & $n t / U n$ & $I D$ & $M W$ & Status & Year & Fuel & Water & Cooling System \\
\hline LABADIE & UNION ELECTRIC CO & MO & 19436 & 2103 & 3 & 621 & Operational & 1972 & COAL & FRESH & ONCE THROUGH \\
\hline LABADIE & UNION ELECTRIC CO & MO & 19436 & 2103 & 4 & 621 & Operational & 1973 & COAL & FRESH & ONCE THROUGH \\
\hline $\begin{array}{l}\text { LAKE } \\
\text { CATHERINE }\end{array}$ & $\begin{array}{l}\text { ARKANSAS POWER } \\
\& \text { LIGHT CO }\end{array}$ & AR & 814 & 170 & 3 & 120 & Operational & 1953 & GAS & FRESH & ONCE THROUGH \\
\hline $\begin{array}{l}\text { LAKE } \\
\text { CATHERINE }\end{array}$ & $\begin{array}{l}\text { ARKANSAS POWER } \\
\& \text { LIGHT CO }\end{array}$ & $A R$ & 814 & 170 & 4 & 553 & Operational & 1970 & GAS & FRESH & ONCE THROUGH \\
\hline $\begin{array}{l}\text { LAKE } \\
\text { CATHERINE }\end{array}$ & $\begin{array}{l}\text { ARKANSAS POWER } \\
\& \text { LIGHT CO }\end{array}$ & AR & 814 & 170 & 1 & 41 & Standby & 1950 & GAS & FRESH & ONCE THROUGH \\
\hline $\begin{array}{l}\text { LAKE } \\
\text { CATHERINE }\end{array}$ & $\begin{array}{l}\text { ARKANSAS POWER } \\
\& \text { LIGHT CO }\end{array}$ & AR & 814 & 170 & 2 & 40 & Standby & 1950 & GAS & FRESH & ONCE THROUGH \\
\hline $\begin{array}{l}\text { LAKE } \\
\text { HUBBARD }\end{array}$ & $\begin{array}{l}\text { TEXAS UTILITIES } \\
\text { ELEC CO }\end{array}$ & TX & 44372 & 3452 & 1 & 397 & Operational & 1970 & GAS & FRESH & ONCE THROUGH \\
\hline $\begin{array}{l}\text { LAKE } \\
\text { HUBBARD }\end{array}$ & $\begin{array}{l}\text { TEXAS UTILITIES } \\
\text { ELEC CO }\end{array}$ & TX & 44372 & 3452 & 2 & 531 & Operational & 1973 & GAS & FRESH & ONCE THROUGH \\
\hline LAKE PAULINE & $\begin{array}{l}\text { WEST TEXAS UTIL } \\
\text { CO }\end{array}$ & TX & 20404 & 3521 & 1 & 20 & Standby & 1928 & GAS & FRESH & ONCE THROUGH \\
\hline LAKE PAULINE & $\begin{array}{l}\text { WEST TEXAS UTIL } \\
\text { CO }\end{array}$ & TX & 20404 & 3521 & 2 & 24.4 & Standby & 1951 & GAS & FRESH & ONCE THROUGH \\
\hline $\begin{array}{l}\text { LAKE ROAD } \\
(\mathrm{MO})\end{array}$ & $\begin{array}{l}\text { ST JOSEPH LIGHT \& } \\
\text { POWER }\end{array}$ & MO & 17881 & 2098 & 4 & 90 & Operational & 1967 & COAL & GROUND & ONCE THROUGH \\
\hline $\begin{array}{l}\text { LAKE ROAD } \\
(\mathrm{OH})\end{array}$ & $\begin{array}{l}\text { CLEVELAND PUBLIC } \\
\text { POWER }\end{array}$ & $\mathrm{OH}$ & 3762 & 2908 & 10 & 25 & Standby & 1953 & COAL & FRESH & ONCE THROUGH \\
\hline $\begin{array}{l}\text { LAKE ROAD } \\
(\mathrm{OH})\end{array}$ & $\begin{array}{l}\text { CLEVELAND PUBLIC } \\
\text { POWER }\end{array}$ & $\mathrm{OH}$ & 3762 & 2908 & 11 & 85 & Standby & 1967 & COAL & FRESH & ONCE THROUGH \\
\hline $\begin{array}{l}\text { LAKE ROAD } \\
(\mathrm{OH})\end{array}$ & $\begin{array}{l}\text { CLEVELAND PUBLIC } \\
\text { POWER }\end{array}$ & $\mathrm{OH}$ & 3762 & 2908 & 8 & 25 & Standby & 1941 & COAL & FRESH & ONCE THROUGH \\
\hline
\end{tabular}




\begin{tabular}{|c|c|c|c|c|c|c|c|c|c|c|c|}
\hline Plant Name & Utility Name & State & Utility/Pl & $n t / U n i$ & $I D$ & $M W$ & Status & Year & Fuel & Water & Cooling System \\
\hline $\begin{array}{l}\text { LAKE ROAD } \\
(\mathrm{OH})\end{array}$ & $\begin{array}{l}\text { CLEVELAND PUBLIC } \\
\text { POWER }\end{array}$ & $\mathrm{OH}$ & 3762 & 2908 & 9 & 25 & Standby & 1953 & COAL & FRESH & ONCE THROUGH \\
\hline LAKESIDE (IL) & $\begin{array}{l}\text { SPRINGFIELD WTR } \\
\text { LT \& PWR }\end{array}$ & IL & 17828 & 964 & 7 & 37.5 & Operational & 1965 & COAL & FRESH & ONCE THROUGH \\
\hline LAKESIDE (IL) & $\begin{array}{l}\text { SPRINGFIELD WTR } \\
\text { LT \& PWR }\end{array}$ & IL & 17828 & 964 & 6 & 37.5 & Operational & 1961 & COAL & FRESH & ONCE THROUGH \\
\hline LAMAR & $\begin{array}{l}\text { LAMAR UTILITIES } \\
\text { BOARD }\end{array}$ & $\mathrm{CO}$ & 10633 & 508 & 4 & 25 & Standby & 1972 & GAS & GROUND & ONCE THROUGH \\
\hline LAMAR & $\begin{array}{l}\text { LAMAR UTILITIES } \\
\text { BOARD }\end{array}$ & $\mathrm{CO}$ & 10633 & 508 & 3 & 5 & Standby & 1952 & GAS & GROUND & ONCE THROUGH \\
\hline LANSING & $\begin{array}{l}\text { INTERSTATE } \\
\text { POWER CO }\end{array}$ & IA & 9392 & 1047 & 1 & 15 & Operational & 1948 & COAL & FRESH & ONCE THROUGH \\
\hline LANSING & $\begin{array}{l}\text { INTERSTATE } \\
\text { POWER CO }\end{array}$ & IA & 9392 & 1047 & 2 & 11.5 & Operational & 1949 & COAL & FRESH & ONCE THROUGH \\
\hline LANSING & $\begin{array}{l}\text { INTERSTATE } \\
\text { POWER CO }\end{array}$ & IA & 9392 & 1047 & 3 & 37.5 & Operational & 1957 & COAL & FRESH & ONCE THROUGH \\
\hline LANSING & $\begin{array}{l}\text { INTERSTATE } \\
\text { POWER CO }\end{array}$ & IA & 9392 & 1047 & 4 & 275 & Operational & 1977 & COAL & FRESH & ONCE THROUGH \\
\hline $\begin{array}{l}\text { LANSING } \\
\text { SMITH }\end{array}$ & GULF POWER CO & $\mathrm{FL}$ & 7801 & 643 & 2 & 190 & Operational & 1967 & COAL & SALINE & ONCE THROUGH \\
\hline $\begin{array}{l}\text { LANSING } \\
\text { SMITH }\end{array}$ & GULF POWER CO & $\mathrm{FL}$ & 7801 & 643 & 1 & 150 & Operational & 1965 & COAL & SALINE & ONCE THROUGH \\
\hline $\begin{array}{l}\text { LARSEN } \\
\text { MEMORIAL }\end{array}$ & $\begin{array}{l}\text { LAKELAND LT \& } \\
\text { WATER DEPT }\end{array}$ & $\mathrm{FL}$ & 10623 & 675 & 7 & 50 & Operational & 1966 & OIL & FRESH & ONCE THROUGH \\
\hline $\begin{array}{l}\text { LARSEN } \\
\text { MEMORIAL }\end{array}$ & $\begin{array}{l}\text { LAKELAND LT \& } \\
\text { WATER DEPT }\end{array}$ & $\mathrm{FL}$ & 10623 & 675 & 6 & 25 & Operational & 1959 & OIL & FRESH & ONCE THROUGH \\
\hline LD WRIGHT & $\begin{array}{l}\text { FREMONT DEPT OF } \\
\text { UTILITIES }\end{array}$ & NE & 6779 & 2240 & 6 & 16 & Operational & 1957 & COAL & GROUND & ONCE THROUGH \\
\hline
\end{tabular}




\begin{tabular}{|c|c|c|c|c|c|c|c|c|c|c|c|}
\hline Plant Name & Utility Name & State & Utility/Pl & $n t / U n$ & $I D$ & $M W$ & Status & Year & Fuel & Water & Cooling System \\
\hline LD WRIGHT & $\begin{array}{l}\text { FREMONT DEPT OF } \\
\text { UTILITIES }\end{array}$ & NE & 6779 & 2240 & 7 & 22 & Operational & 1963 & COAL & GROUND & ONCE THROUGH \\
\hline LEE (SC) & DUKE POWER CO & SC & 5416 & 3264 & 1 & 90 & Operational & 1951 & COAL & FRESH & $\begin{array}{l}\text { COMBINATION (ONCE } \\
\text { THROUGH/HELPER TOWER) }\end{array}$ \\
\hline LEE (SC) & DUKE POWER CO & SC & 5416 & 3264 & 2 & 90 & Operational & 1951 & COAL & FRESH & $\begin{array}{l}\text { COMBINATION (ONCE } \\
\text { THROUGH/HELPER TOWER) }\end{array}$ \\
\hline LEE (SC) & DUKE POWER CO & SC & 5416 & 3264 & 3 & 165 & Operational & 1958 & COAL & FRESH & $\begin{array}{l}\text { COMBINATION (ONCE } \\
\text { THROUGH/HELPER TOWER) }\end{array}$ \\
\hline LELAND OLDS & $\begin{array}{l}\text { BASIN ELEC } \\
\text { POWER COOP }\end{array}$ & ND & 1307 & 2817 & 1 & 216 & Operational & 1966 & COAL & FRESH & ONCE THROUGH \\
\hline LELAND OLDS & $\begin{array}{l}\text { BASIN ELEC } \\
\text { POWER COOP }\end{array}$ & ND & 1307 & 2817 & 2 & 440 & Operational & 1975 & COAL & FRESH & ONCE THROUGH \\
\hline $\begin{array}{l}\text { LEWIS \& } \\
\text { CLARK }\end{array}$ & $\begin{array}{l}\text { MONTANA-DAKOTA } \\
\text { UTILITIES }\end{array}$ & MT & 12819 & 6089 & 1 & 50 & Operational & 1958 & COAL & FRESH & ONCE THROUGH \\
\hline LINDEN & $\begin{array}{l}\text { PUBLIC SERVICE } \\
\text { ELEC \& GAS }\end{array}$ & $\mathrm{NJ}$ & 15477 & 2406 & 1 & 260 & Operational & 1957 & OIL & SALINE & ONCE THROUGH \\
\hline LINDEN & $\begin{array}{l}\text { PUBLIC SERVICE } \\
\text { ELEC \& GAS }\end{array}$ & NJ & 15477 & 2406 & 2 & 260 & Operational & 1957 & OIL & SALINE & ONCE THROUGH \\
\hline LITTLE GYPSY & $\begin{array}{l}\text { LOUISIANA POWER } \\
\text { \& LIGHT }\end{array}$ & LA & 11241 & 1402 & 3 & 582 & Operational & 1969 & GAS & FRESH & ONCE THROUGH \\
\hline LITTLE GYPSY & $\begin{array}{l}\text { LOUISIANA POWER } \\
\text { \& LIGHT }\end{array}$ & LA & 11241 & 1402 & 2 & 383 & Operational & 1965 & GAS & FRESH & ONCE THROUGH \\
\hline LITTLE GYPSY & $\begin{array}{l}\text { LOUISIANA POWER } \\
\& \text { LIGHT }\end{array}$ & LA & 11241 & 1402 & 1 & 225 & Operational & 1961 & GAS & FRESH & ONCE THROUGH \\
\hline LL WILKINS & $\begin{array}{l}\text { CLARKSDALE PUB } \\
\text { UTIL COMM }\end{array}$ & MS & 3702 & 2059 & 6 & 6 & Standby & 1956 & GAS & GROUND & ONCE THROUGH \\
\hline LL WILKINS & $\begin{array}{l}\text { CLARKSDALE PUB } \\
\text { UTIL COMM }\end{array}$ & MS & 3702 & 2059 & 7 & 9 & Standby & 1965 & GAS & GROUND & ONCE THROUGH \\
\hline
\end{tabular}




\begin{tabular}{|c|c|c|c|c|c|c|c|c|c|c|c|}
\hline Plant Name & Utility Name & State & Utility/Pl & nt/Uni & $I D$ & $M W$ & Status & Year & Fuel & Water & Cooling System \\
\hline LOGANSPORT & $\begin{array}{l}\text { LOGANSPORT MUNI } \\
\text { UTIL }\end{array}$ & IN & 11142 & 1032 & 4 & 18 & Operational & 1958 & COAL & FRESH & ONCE THROUGH \\
\hline LOGANSPORT & $\begin{array}{l}\text { LOGANSPORT MUNI } \\
\text { UTIL }\end{array}$ & IN & 11142 & 1032 & 5 & 25 & Operational & 1964 & COAL & FRESH & ONCE THROUGH \\
\hline LOVETT & $\begin{array}{l}\text { ORANGE \& } \\
\text { ROCKLAND UTIL }\end{array}$ & NY & 14154 & 2629 & 3 & 69 & Operational & 1955 & GAS & BRACKISH & ONCE THROUGH \\
\hline LOVETT & $\begin{array}{l}\text { ORANGE \& } \\
\text { ROCKLAND UTIL }\end{array}$ & NY & 14154 & 2629 & 4 & 180 & Operational & 1966 & COAL & BRACKISH & ONCE THROUGH \\
\hline LOVETT & $\begin{array}{l}\text { ORANGE \& } \\
\text { ROCKLAND UTIL }\end{array}$ & NY & 14154 & 2629 & 2 & 23 & Standby & 1951 & GAS & BRACKISH & ONCE THROUGH \\
\hline LOVETT & $\begin{array}{l}\text { ORANGE \& } \\
\text { ROCKLAND UTIL }\end{array}$ & NY & 14154 & 2629 & 1 & 23 & Standby & 1949 & GAS & BRACKISH & ONCE THROUGH \\
\hline LOVETT & $\begin{array}{l}\text { ORANGE \& } \\
\text { ROCKLAND UTIL }\end{array}$ & NY & 14154 & 2629 & 5 & 201 & Operational & 1969 & COAL & BRACKISH & ONCE THROUGH \\
\hline MADGETT & $\begin{array}{l}\text { DAIRYLAND POWER } \\
\text { COOP }\end{array}$ & WI & 4716 & 4271 & 1 & 387 & Operational & 1979 & COAL & FRESH & ONCE THROUGH \\
\hline $\begin{array}{l}\text { MANCHESTER } \\
\text { STREET }\end{array}$ & $\begin{array}{l}\text { NEW ENGLAND } \\
\text { POWER CO }\end{array}$ & $\mathrm{RI}$ & 13433 & 3236 & 10 & 46 & Operational & 1947 & GAS & BRACKISH & ONCE THROUGH \\
\hline $\begin{array}{l}\text { MANCHESTER } \\
\text { STREET }\end{array}$ & $\begin{array}{l}\text { NEW ENGLAND } \\
\text { POWER CO }\end{array}$ & $\mathrm{RI}$ & 13433 & 3236 & 11 & 46 & Operational & 1949 & OIL & BRACKISH & ONCE THROUGH \\
\hline $\begin{array}{l}\text { MANCHESTER } \\
\text { STREET }\end{array}$ & $\begin{array}{l}\text { NEW ENGLAND } \\
\text { POWER CO }\end{array}$ & $\mathrm{RI}$ & 13433 & 3236 & 9 & 46 & Operational & 1941 & GAS & BRACKISH & ONCE THROUGH \\
\hline MANDALAY & $\begin{array}{l}\text { SOUTHERN CALIF } \\
\text { EDISON CO }\end{array}$ & $\mathrm{CA}$ & 17609 & 345 & 1 & 218 & Operational & 1959 & GAS & SALINE & ONCE THROUGH \\
\hline MANDALAY & $\begin{array}{l}\text { SOUTHERN CALIF } \\
\text { EDISON CO }\end{array}$ & $\mathrm{CA}$ & 17609 & 345 & 2 & 218 & Operational & 1959 & GAS & SALINE & ONCE THROUGH \\
\hline MANITOWOC & $\begin{array}{l}\text { MANITOWOC } \\
\text { PUBLIC UTIL }\end{array}$ & WI & 11571 & 4125 & 3 & 10 & Standby & 1941 & COAL & FRESH & ONCE THROUGH \\
\hline
\end{tabular}




\begin{tabular}{|c|c|c|c|c|c|c|c|c|c|c|c|}
\hline Plant Name & Utility Name & State & Utility/Pl & tt/Uni & $I D$ & $M W$ & Status & Year & Fuel & Water & Cooling Systen \\
\hline MANITOWOC & $\begin{array}{l}\text { MANITOWOC } \\
\text { PUBLIC UTIL }\end{array}$ & WI & 11571 & 4125 & 7 & 21 & Operational & 1964 & COAL & FRESH & ONCE THROUGH \\
\hline MANITOWOC & $\begin{array}{l}\text { MANITOWOC } \\
\text { PUBLIC UTIL }\end{array}$ & WI & 11571 & 4125 & 6 & 32 & Operational & 1956 & COAL & FRESH & ONCE THROUGH \\
\hline MANITOWOC & $\begin{array}{l}\text { MANITOWOC } \\
\text { PUBLIC UTIL }\end{array}$ & WI & 11571 & 4125 & 4 & 10 & Standby & 1946 & COAL & FRESH & ONCE THROUGH \\
\hline MANITOWOC & $\begin{array}{l}\text { MANITOWOC } \\
\text { PUBLIC UTIL }\end{array}$ & WI & 11571 & 4125 & 5 & 22 & Operational & 1952 & COAL & FRESH & ONCE THROUGH \\
\hline MARION (IL) & $\begin{array}{l}\text { SOUTH ILLINOIS } \\
\text { POWER COOP }\end{array}$ & IL & 17632 & 976 & 1 & 33 & Operational & 1963 & COAL & FRESH & ONCE THROUGH \\
\hline MARION (IL) & $\begin{array}{l}\text { SOUTH ILLINOIS } \\
\text { POWER COOP }\end{array}$ & IL & 17632 & 976 & 2 & 33 & Operational & 1963 & COAL & FRESH & ONCE THROUGH \\
\hline MARION (IL) & $\begin{array}{l}\text { SOUTH ILLINOIS } \\
\text { POWER COOP }\end{array}$ & IL & 17632 & 976 & 3 & 33 & Operational & 1963 & COAL & FRESH & ONCE THROUGH \\
\hline MARION (IL) & $\begin{array}{l}\text { SOUTH ILLINOIS } \\
\text { POWER COOP }\end{array}$ & IL & 17632 & 976 & 4 & 173 & Operational & 1978 & COAL & FRESH & ONCE THROUGH \\
\hline $\begin{array}{l}\text { MARSHALL } \\
\text { (NC) }\end{array}$ & DUKE POWER CO & NC & 5416 & 2727 & 1 & 350 & Operational & 1965 & COAL & FRESH & ONCE THROUGH \\
\hline $\begin{array}{l}\text { MARSHALL } \\
\text { (NC) }\end{array}$ & DUKE POWER CO & $\mathrm{NC}$ & 5416 & 2727 & 2 & 350 & Operational & 1966 & COAL & FRESH & ONCE THROUGH \\
\hline $\begin{array}{l}\text { MARSHALL } \\
\text { (NC) }\end{array}$ & DUKE POWER CO & NC & 5416 & 2727 & 3 & 648 & Operational & 1969 & COAL & FRESH & ONCE THROUGH \\
\hline $\begin{array}{l}\text { MARSHALL } \\
\text { (NC) }\end{array}$ & DUKE POWER CO & $\mathrm{NC}$ & 5416 & 2727 & 4 & 648 & Operational & 1970 & COAL & FRESH & ONCE THROUGH \\
\hline $\begin{array}{l}\text { MARTINS } \\
\text { CREEK }\end{array}$ & $\begin{array}{l}\text { PENNSYLVANIA } \\
\text { POWER \& LT }\end{array}$ & PA & 14715 & 3148 & 1 & 156 & Operational & 1954 & COAL & FRESH & ONCE THROUGH \\
\hline $\begin{array}{l}\text { MARTINS } \\
\text { CREEK }\end{array}$ & $\begin{array}{l}\text { PENNSYLVANIA } \\
\text { POWER \& LT }\end{array}$ & PA & 14715 & 3148 & 2 & 156 & Operational & 1956 & COAL & FRESH & ONCE THROUGH \\
\hline
\end{tabular}




\begin{tabular}{|c|c|c|c|c|c|c|c|c|c|c|c|}
\hline Plant Name & Utility Name & State & Utility/Pl & $n t / U n$ & $I D$ & $M W$ & Status & Year & Fuel & Water & Cooling System \\
\hline MARYSVILLE & $\begin{array}{l}\text { DETROIT EDISON } \\
\text { CO }\end{array}$ & MI & 5109 & 1732 & 8 & 75 & Operational & 1947 & COAL & FRESH & ONCE THROUGH \\
\hline MARYSVILLE & $\begin{array}{l}\text { DETROIT EDISON } \\
\text { CO }\end{array}$ & $\mathrm{Ml}$ & 5109 & 1732 & 6 & 50 & Operational & 1942 & COAL & FRESH & ONCE THROUGH \\
\hline MARYSVILLE & $\begin{array}{l}\text { DETROIT EDISON } \\
\text { CO }\end{array}$ & MI & 5109 & 1732 & 7 & 75 & Operational & 1943 & COAL & FRESH & ONCE THROUGH \\
\hline MCCLELLAN & $\begin{array}{l}\text { ARKANSAS ELEC } \\
\text { COOP CORP }\end{array}$ & AR & 807 & 203 & 1 & 133 & Operational & 1972 & GAS & FRESH & $\begin{array}{l}\text { COMBINATION (ONCE } \\
\text { THROUGH/SPRAY POND) }\end{array}$ \\
\hline MCDONOUGH & $\begin{array}{l}\text { GEORGIA POWER } \\
\text { CO }\end{array}$ & GA & 7140 & 710 & 1 & 299 & Operational & 1963 & COAL & FRESH & ONCE THROUGH \\
\hline MCDONOUGH & $\begin{array}{l}\text { GEORGIA POWER } \\
\text { CO }\end{array}$ & GA & 7140 & 710 & 2 & 299 & Operational & 1964 & COAL & FRESH & ONCE THROUGH \\
\hline MCGUIRE & DUKE POWER CO & $\mathrm{NC}$ & 5416 & 6038 & 1 & 1305 & Operational & 1981 & UR & FRESH & ONCE THROUGH \\
\hline MCGUIRE & DUKE POWER CO & $\mathrm{NC}$ & 5416 & 6038 & 2 & 1305 & Operational & 1984 & UR & FRESH & ONCE THROUGH \\
\hline MCINTOSH (FL) & $\begin{array}{l}\text { LAKELAND LT \& } \\
\text { WATER DEPT }\end{array}$ & $\mathrm{FL}$ & 10623 & 676 & 1 & 90 & Operational & 1971 & OIL & FRESH & ONCE THROUGH \\
\hline $\begin{array}{l}\text { MCINTOSH } \\
\text { (GA) }\end{array}$ & $\begin{array}{l}\text { SAVANNAH ELEC \& } \\
\text { POWER CO }\end{array}$ & GA & 16687 & 6124 & 1 & 178 & Operational & 1979 & COAL & FRESH & ONCE THROUGH \\
\hline MCMANUS & $\begin{array}{l}\text { GEORGIA POWER } \\
\text { CO }\end{array}$ & GA & 7140 & 715 & 1 & 50 & Operational & 1952 & OIL & BRACKISH & ONCE THROUGH \\
\hline MCMANUS & $\begin{array}{l}\text { GEORGIA POWER } \\
\text { CO }\end{array}$ & GA & 7140 & 715 & 2 & 93.8 & Operational & 1959 & OIL & BRACKISH & ONCE THROUGH \\
\hline MCMEEKIN & $\begin{array}{l}\text { SOUTH CAROLINA } \\
\text { ELEC \& GAS }\end{array}$ & SC & 17539 & 3287 & 1 & 147 & Operational & 1958 & COAL & FRESH & ONCE THROUGH \\
\hline MCMEEKIN & $\begin{array}{l}\text { SOUTH CAROLINA } \\
\text { ELEC \& GAS }\end{array}$ & SC & 17539 & 3287 & 2 & 147 & Operational & 1958 & COAL & FRESH & ONCE THROUGH \\
\hline
\end{tabular}




\begin{tabular}{|c|c|c|c|c|c|c|c|c|c|c|c|}
\hline Plant Name & Utility Name & State & Utility/Pl & $n t / U n$ & $I D$ & $M W$ & Status & Year & Fuel & Water & Cooling System \\
\hline MCWILLIAMS & $\begin{array}{l}\text { ALABAMA ELECTRIC } \\
\text { COOP }\end{array}$ & $\mathrm{AL}$ & 189 & 533 & 1 & 7.5 & Operational & 1954 & GAS & FRESH & ONCE THROUGH \\
\hline MCWILLIAMS & $\begin{array}{l}\text { ALABAMA ELECTRIC } \\
\text { COOP }\end{array}$ & $\mathrm{AL}$ & 189 & 533 & 3 & 25 & Operational & 1959 & GAS & FRESH & ONCE THROUGH \\
\hline MCWILLIAMS & $\begin{array}{l}\text { ALABAMA ELECTRIC } \\
\text { COOP }\end{array}$ & $A L$ & 189 & 533 & 2 & 7.5 & Operational & 1954 & GAS & FRESH & ONCE THROUGH \\
\hline MERAMEC & UNION ELECTRIC CO & MO & 19436 & 2104 & 1 & 138 & Operational & 1953 & COAL & FRESH & ONCE THROUGH \\
\hline MERAMEC & UNION ELECTRIC CO & MO & 19436 & 2104 & 2 & 138 & Operational & 1954 & COAL & FRESH & ONCE THROUGH \\
\hline MERAMEC & UNION ELECTRIC CO & MO & 19436 & 2104 & 3 & 289 & Operational & 1959 & COAL & FRESH & ONCE THROUGH \\
\hline MERAMEC & UNION ELECTRIC CO & MO & 19436 & 2104 & 4 & 359 & Operational & 1961 & COAL & FRESH & ONCE THROUGH \\
\hline MERCER & $\begin{array}{l}\text { PUBLIC SERVICE } \\
\text { ELEC \& GAS }\end{array}$ & NJ & 15477 & 2408 & 1 & 326 & Operational & 1960 & COAL & FRESH & ONCE THROUGH \\
\hline MERCER & $\begin{array}{l}\text { PUBLIC SERVICE } \\
\text { ELEC \& GAS }\end{array}$ & NJ & 15477 & 2408 & 2 & 326 & Operational & 1961 & COAL & FRESH & ONCE THROUGH \\
\hline MEREDOSIA & $\begin{array}{l}\text { CENT ILLINOIS } \\
\text { PUBLIC SERV }\end{array}$ & IL & 3253 & 864 & 1 & 57.5 & Operational & 1948 & COAL & FRESH & ONCE THROUGH \\
\hline MEREDOSIA & $\begin{array}{l}\text { CENT ILLINOIS } \\
\text { PUBLIC SERV }\end{array}$ & IL & 3253 & 864 & 2 & 57.5 & Operational & 1949 & COAL & FRESH & ONCE THROUGH \\
\hline MEREDOSIA & $\begin{array}{l}\text { CENT ILLINOIS } \\
\text { PUBLIC SERV }\end{array}$ & IL & 3253 & 864 & 3 & 239 & Operational & 1960 & COAL & FRESH & ONCE THROUGH \\
\hline MERRIMACK & $\begin{array}{l}\text { PUBLIC SERV NEW } \\
\text { HAMPSHIRE }\end{array}$ & $\mathrm{NH}$ & 15472 & 2364 & 1 & 114 & Operational & 1960 & COAL & FRESH & $\begin{array}{l}\text { COMBINATION (ONCE } \\
\text { THROUGH/SPRAY CANAL) }\end{array}$ \\
\hline MERRIMACK & $\begin{array}{l}\text { PUBLIC SERV NEW } \\
\text { HAMPSHIRE }\end{array}$ & $\mathrm{NH}$ & 15472 & 2364 & 2 & 346 & Operational & 1968 & COAL & FRESH & $\begin{array}{l}\text { COMBINATION (ONCE } \\
\text { THROUGH/SPRAY CANAL) }\end{array}$ \\
\hline
\end{tabular}




\begin{tabular}{|c|c|c|c|c|c|c|c|c|c|c|c|}
\hline Plant Name & Utility Name & State & Utility/P & $n t / U n i$ & $I D$ & $M W$ & Status & Year & Fuel & Water & Cooling System \\
\hline MIAMI FORT & $\begin{array}{l}\text { CINCINNATI GAS \& } \\
\text { ELEC CO }\end{array}$ & $\mathrm{OH}$ & 3542 & 2832 & 5 & 100 & Operational & 1949 & COAL & FRESH & ONCE THROUGH \\
\hline MIAMI FORT & $\begin{array}{l}\text { CINCINNATI GAS \& } \\
\text { ELEC CO }\end{array}$ & $\mathrm{OH}$ & 3542 & 2832 & 6 & 163 & Operational & 1960 & COAL & FRESH & ONCE THROUGH \\
\hline MICHIGAN CITY & $\begin{array}{l}\text { NO INDIANA PUBLIC } \\
\text { SERVICE }\end{array}$ & IN & 13756 & 997 & 2 & 70 & Operational & 1950 & GAS & FRESH & ONCE THROUGH \\
\hline MICHIGAN CITY & $\begin{array}{l}\text { NO INDIANA PUBLIC } \\
\text { SERVICE }\end{array}$ & IN & 13756 & 997 & 3 & 70 & Operational & 1951 & GAS & FRESH & ONCE THROUGH \\
\hline MICHOUD & $\begin{array}{l}\text { NEW ORLEANS } \\
\text { PUBLIC SERV }\end{array}$ & LA & 13478 & 1409 & 3 & 582 & Operational & 1967 & GAS & BRACKISH & ONCE THROUGH \\
\hline MICHOUD & $\begin{array}{l}\text { NEW ORLEANS } \\
\text { PUBLIC SERV }\end{array}$ & LA & 13478 & 1409 & 1 & 115 & Standby & 1957 & GAS & BRACKISH & ONCE THROUGH \\
\hline MICHOUD & $\begin{array}{l}\text { NEW ORLEANS } \\
\text { PUBLIC SERV }\end{array}$ & LA & 13478 & 1409 & 2 & 238 & Operational & 1963 & GAS & BRACKISH & ONCE THROUGH \\
\hline MIDDLETOWN & $\begin{array}{l}\text { CONNECTICUT } \\
\text { LIGHT \& POWER }\end{array}$ & CT & 4176 & 562 & 2 & 114 & Operational & 1958 & OIL & FRESH & ONCE THROUGH \\
\hline MIDDLETOWN & $\begin{array}{l}\text { CONNECTICUT } \\
\text { LIGHT \& POWER }\end{array}$ & CT & 4176 & 562 & 3 & 239 & Operational & 1964 & OIL & FRESH & ONCE THROUGH \\
\hline $\begin{array}{l}\text { MILL CREEK } \\
(\mathrm{KY})\end{array}$ & $\begin{array}{l}\text { LOUISVILLE GAS \& } \\
\text { ELEC CO }\end{array}$ & KY & 11249 & 1364 & 1 & 356 & Operational & 1972 & COAL & FRESH & ONCE THROUGH \\
\hline MILLIKEN & $\begin{array}{l}\text { NEW YORK STATE } \\
\text { ELEC \& GAS }\end{array}$ & NY & 13511 & 2535 & 1 & 155 & Operational & 1955 & COAL & FRESH & ONCE THROUGH \\
\hline MILLIKEN & $\begin{array}{l}\text { NEW YORK STATE } \\
\text { ELEC \& GAS }\end{array}$ & NY & 13511 & 2535 & 2 & 167 & Operational & 1958 & COAL & FRESH & ONCE THROUGH \\
\hline MILLSTONE & $\begin{array}{l}\text { NORTHEAST NUC } \\
\text { ENERGY CO }\end{array}$ & $\mathrm{CT}$ & 21687 & 566 & 1 & 662 & Operational & 1971 & UR & SALINE & ONCE THROUGH \\
\hline MILLSTONE & $\begin{array}{l}\text { NORTHEAST NUC } \\
\text { ENERGY CO }\end{array}$ & $\mathrm{CT}$ & 21687 & 566 & 2 & 909 & Operational & 1975 & UR & SALINE & ONCE THROUGH \\
\hline
\end{tabular}




\begin{tabular}{|c|c|c|c|c|c|c|c|c|c|c|c|}
\hline Plant Name & Utility Name & State & Utility/Pl & $n t / U n i$ & $I D$ & $\boldsymbol{M W}$ & Status & Year & Fuel & Water & Cooling System \\
\hline MILLSTONE & $\begin{array}{l}\text { NORTHEAST NUC } \\
\text { ENERGY CO }\end{array}$ & CT & 21687 & 566 & 3 & 1253 & Operational & 1986 & UR & SALINE & ONCE THROUGH \\
\hline $\begin{array}{l}\text { MINNESOTA } \\
\text { VALLEY }\end{array}$ & $\begin{array}{l}\text { NORTHERN STATES } \\
\text { POWER CO }\end{array}$ & $\mathrm{MN}$ & 13781 & 1918 & 3 & 46 & Operational & 1953 & COAL & FRESH & ONCE THROUGH \\
\hline MISSOURI CITY & $\begin{array}{l}\text { INDEPENDENCE } \\
\text { POWER \& LT }\end{array}$ & MO & 9231 & 2171 & 1 & 23 & Operational & 1954 & COAL & FRESH & ONCE THROUGH \\
\hline MISSOURI CITY & $\begin{array}{l}\text { INDEPENDENCE } \\
\text { POWER \& LT }\end{array}$ & MO & 9231 & 2171 & 2 & 23 & Operational & 1954 & COAL & FRESH & ONCE THROUGH \\
\hline MISTERSKY & $\begin{array}{l}\text { DETROIT PUBLIC } \\
\text { LIGHTING }\end{array}$ & $\mathrm{Ml}$ & 5107 & 1822 & 5 & 44 & Operational & 1950 & OIL & FRESH & ONCE THROUGH \\
\hline MISTERSKY & $\begin{array}{l}\text { DETROIT PUBLIC } \\
\text { LIGHTING }\end{array}$ & $\mathrm{Ml}$ & 5107 & 1822 & 6 & 50 & Operational & 1958 & OIL & FRESH & ONCE THROUGH \\
\hline MISTERSKY & $\begin{array}{l}\text { DETROIT PUBLIC } \\
\text { LIGHTING }\end{array}$ & Ml & 5107 & 1822 & 7 & 60 & Operational & 1979 & GAS & FRESH & ONCE THROUGH \\
\hline MITCHELL (GA) & $\begin{array}{l}\text { GEORGIA POWER } \\
\text { CO }\end{array}$ & GA & 7140 & 727 & 1 & 27.6 & Operational & 1948 & COAL & FRESH & ONCE THROUGH \\
\hline MITCHELL (GA) & $\begin{array}{l}\text { GEORGIA POWER } \\
\text { CO }\end{array}$ & GA & 7140 & 727 & 2 & 27.6 & Operational & 1949 & COAL & FRESH & ONCE THROUGH \\
\hline MITCHELL (GA) & $\begin{array}{l}\text { GEORGIA POWER } \\
\text { CO }\end{array}$ & GA & 7140 & 727 & 3 & 163 & Operational & 1964 & COAL & FRESH & ONCE THROUGH \\
\hline MITCHELL (PA) & $\begin{array}{l}\text { WEST PENN } \\
\text { POWER CO }\end{array}$ & PA & 20387 & 3181 & 1 & 74.8 & Operational & 1948 & OIL & FRESH & ONCE THROUGH \\
\hline MITCHELL (PA) & $\begin{array}{l}\text { WEST PENN } \\
\text { POWER CO }\end{array}$ & PA & 20387 & 3181 & 2 & 74.8 & Standby & 1949 & OIL & FRESH & ONCE THROUGH \\
\hline MITCHELL (PA) & $\begin{array}{l}\text { WEST PENN } \\
\text { POWER CO }\end{array}$ & PA & 20387 & 3181 & 3 & 299 & Operational & 1963 & COAL & FRESH & ONCE THROUGH \\
\hline ML HIBBARD & $\begin{array}{l}\text { MINNESOTA } \\
\text { POWER \& LIGHT }\end{array}$ & MN & 12647 & 1897 & 1 & 25 & Standby & 1931 & OIL & FRESH & ONCE THROUGH \\
\hline
\end{tabular}




\begin{tabular}{|c|c|c|c|c|c|c|c|c|c|c|c|}
\hline Plant Name & Utility Name & State & Utility/Pl & th/Uni & $I D$ & $M W$ & Status & Year & Fuel & Water & Cooling System \\
\hline ML HIBBARD & $\begin{array}{l}\text { MINNESOTA } \\
\text { POWER \& LIGHT }\end{array}$ & $\mathrm{MN}$ & 12647 & 1897 & 4 & 37.5 & Operational & 1951 & WOOD & FRESH & ONCE THROUGH \\
\hline ML HIBBARD & $\begin{array}{l}\text { MINNESOTA } \\
\text { POWER \& LIGHT }\end{array}$ & MN & 12647 & 1897 & 2 & 25 & Standby & 1943 & OIL & FRESH & ONCE THROUGH \\
\hline ML HIBBARD & $\begin{array}{l}\text { MINNESOTA } \\
\text { POWER \& LIGHT }\end{array}$ & MN & 12647 & 1897 & 3 & 35 & Operational & 1949 & WOOD & FRESH & ONCE THROUGH \\
\hline ML KAPP & $\begin{array}{l}\text { INTERSTATE } \\
\text { POWER CO }\end{array}$ & IA & 9392 & 1048 & 1 & 18.8 & Operational & 1947 & GAS & FRESH & ONCE THROUGH \\
\hline ML KAPP & $\begin{array}{l}\text { INTERSTATE } \\
\text { POWER CO }\end{array}$ & IA & 9392 & 1048 & 2 & 219 & Operational & 1967 & COAL & FRESH & ONCE THROUGH \\
\hline MONROE (MI) & $\begin{array}{l}\text { DETROIT EDISON } \\
\text { CO }\end{array}$ & $\mathrm{Ml}$ & 5109 & 1733 & 3 & 823 & Operational & 1973 & COAL & FRESH & ONCE THROUGH \\
\hline MONROE (MI) & $\begin{array}{l}\text { DETROIT EDISON } \\
\text { CO }\end{array}$ & Ml & 5109 & 1733 & 4 & 817 & Operational & 1974 & COAL & FRESH & ONCE THROUGH \\
\hline MONROE (MI) & $\begin{array}{l}\text { DETROIT EDISON } \\
\text { CO }\end{array}$ & $\mathrm{Ml}$ & 5109 & 1733 & 1 & 817 & Operational & 1971 & COAL & FRESH & ONCE THROUGH \\
\hline MONROE (MI) & $\begin{array}{l}\text { DETROIT EDISON } \\
\text { CO }\end{array}$ & Ml & 5109 & 1733 & 2 & 823 & Operational & 1973 & COAL & FRESH & ONCE THROUGH \\
\hline $\begin{array}{l}\text { MONTICELLO } \\
\text { (MN) }\end{array}$ & $\begin{array}{l}\text { NORTHERN STATES } \\
\text { POWER CO }\end{array}$ & MN & 13781 & 1922 & 1 & 569 & Operational & 1971 & UR & FRESH & $\begin{array}{l}\text { MIXED MODE (ONCE } \\
\text { THROUGH/COOLING TOWER) }\end{array}$ \\
\hline MONTVILLE & $\begin{array}{l}\text { CONNECTICUT } \\
\text { LIGHT \& POWER }\end{array}$ & CT & 4176 & 546 & 5 & 75 & Operational & 1954 & OIL & BRACKISH & ONCE THROUGH \\
\hline MONTVILLE & $\begin{array}{l}\text { CONNECTICUT } \\
\text { LIGHT \& POWER }\end{array}$ & CT & 4176 & 546 & 6 & 415 & Operational & 1971 & OIL & BRACKISH & ONCE THROUGH \\
\hline $\begin{array}{l}\text { MORGANTOW } \\
\mathrm{N}\end{array}$ & $\begin{array}{l}\text { POTOMAC } \\
\text { ELECTRIC POWER }\end{array}$ & MD & 15270 & 1573 & 1 & 626 & Operational & 1970 & COAL & BRACKISH & ONCE THROUGH \\
\hline $\begin{array}{l}\text { MORGANTOW } \\
\text { N }\end{array}$ & $\begin{array}{l}\text { POTOMAC } \\
\text { ELECTRIC POWER }\end{array}$ & MD & 15270 & 1573 & 2 & 626 & Operational & 1971 & COAL & BRACKISH & ONCE THROUGH \\
\hline
\end{tabular}




\begin{tabular}{|c|c|c|c|c|c|c|c|c|c|c|c|}
\hline \multirow{2}{*}{$\frac{\text { Plant Name }}{\text { MORRO BAY }}$} & \multirow{2}{*}{$\begin{array}{l}\text { Utility Name } \\
\text { PACIFIC GAS \& } \\
\text { ELEC CO }\end{array}$} & \multirow{2}{*}{$\frac{\text { State }}{\mathrm{CA}}$} & \multicolumn{3}{|c|}{ Utility/Plant/Unit ID } & \multirow{2}{*}{$\frac{\boldsymbol{M W}}{169}$} & \multirow{2}{*}{$\frac{\text { Status }}{\text { Operational }}$} & \multirow{2}{*}{$\begin{array}{r}\text { Year } \\
1955\end{array}$} & \multirow{2}{*}{$\frac{\text { Fuel }}{\text { GAS }}$} & \multirow{2}{*}{$\begin{array}{l}\text { Water } \\
\text { SALINE }\end{array}$} & \multirow{2}{*}{$\frac{\text { Cooling System }}{\text { ONCE THROUGH }}$} \\
\hline & & & 14328 & 259 & 2 & & & & & & \\
\hline MORRO BAY & $\begin{array}{l}\text { PACIFIC GAS \& } \\
\text { ELEC CO }\end{array}$ & $\mathrm{CA}$ & 14328 & 259 & 3 & 359 & Operational & 1962 & GAS & SALINE & ONCE THROUGH \\
\hline MORRO BAY & $\begin{array}{l}\text { PACIFIC GAS \& } \\
\text { ELEC CO }\end{array}$ & $\mathrm{CA}$ & 14328 & 259 & 4 & 359 & Operational & 1963 & GAS & SALINE & ONCE THROUGH \\
\hline MORRO BAY & $\begin{array}{l}\text { PACIFIC GAS \& } \\
\text { ELEC CO }\end{array}$ & CA & 14328 & 259 & 1 & 169 & Operational & 1956 & GAS & SALINE & ONCE THROUGH \\
\hline $\begin{array}{l}\text { MOSS } \\
\text { LANDING }\end{array}$ & $\begin{array}{l}\text { PACIFIC GAS \& } \\
\text { ELEC CO }\end{array}$ & $\mathrm{CA}$ & 14328 & 260 & 6 & 812 & Operational & 1967 & GAS & SALINE & ONCE THROUGH \\
\hline $\begin{array}{l}\text { MOSS } \\
\text { LANDING }\end{array}$ & $\begin{array}{l}\text { PACIFIC GAS \& } \\
\text { ELEC CO }\end{array}$ & CA & 14328 & 260 & 7 & 812 & Operational & 1968 & GAS & SALINE & ONCE THROUGH \\
\hline MOUNT STORM & $\begin{array}{l}\text { VIRGINIA ELEC \& } \\
\text { POWER CO }\end{array}$ & WV & 19876 & 3954 & 1 & 570 & Operational & 1965 & COAL & FRESH & ONCE THROUGH \\
\hline MOUNT STORM & $\begin{array}{l}\text { VIRGINIA ELEC \& } \\
\text { POWER CO }\end{array}$ & WV & 19876 & 3954 & 2 & 570 & Operational & 1966 & COAL & FRESH & ONCE THROUGH \\
\hline MOUNT STORM & $\begin{array}{l}\text { VIRGINIA ELEC \& } \\
\text { POWER CO }\end{array}$ & WV & 19876 & 3954 & 3 & 522 & Operational & 1973 & COAL & FRESH & ONCE THROUGH \\
\hline MOUNT TOM & $\begin{array}{l}\text { HOLYOKE WATER } \\
\text { POWER CO }\end{array}$ & MA & 8779 & 1606 & 1 & 136 & Operational & 1960 & COAL & FRESH & ONCE THROUGH \\
\hline $\begin{array}{l}\text { MOUNTAIN } \\
\text { CREEK }\end{array}$ & $\begin{array}{l}\text { TEXAS UTILITIES } \\
\text { ELEC CO }\end{array}$ & TX & 44372 & 3453 & 3 & 75 & Operational & 1949 & GAS & FRESH & ONCE THROUGH \\
\hline $\begin{array}{l}\text { MOUNTAIN } \\
\text { CREEK }\end{array}$ & $\begin{array}{l}\text { TEXAS UTILITIES } \\
\text { ELEC CO }\end{array}$ & $\mathrm{TX}$ & 44372 & 3453 & 8 & 581 & Operational & 1967 & GAS & FRESH & ONCE THROUGH \\
\hline $\begin{array}{l}\text { MOUNTAIN } \\
\text { CREEK }\end{array}$ & $\begin{array}{l}\text { TEXAS UTILITIES } \\
\text { ELEC CO }\end{array}$ & $\mathrm{TX}$ & 44372 & 3453 & 6 & 136 & Operational & 1956 & GAS & FRESH & ONCE THROUGH \\
\hline $\begin{array}{l}\text { MOUNTAIN } \\
\text { CREEK }\end{array}$ & $\begin{array}{l}\text { TEXAS UTILITIES } \\
\text { ELEC CO }\end{array}$ & $\mathrm{TX}$ & 44372 & 3453 & 2 & 31.2 & Standby & 1945 & GAS & FRESH & ONCE THROUGH \\
\hline
\end{tabular}




\begin{tabular}{|c|c|c|c|c|c|c|c|c|c|c|c|}
\hline Plant Name & Utility Name & State & Utility/P & $n t / U n$ & $I D$ & $\boldsymbol{M W}$ & Status & Year & Fuel & Water & Cooling System \\
\hline $\begin{array}{l}\text { MOUNTAIN } \\
\text { CREEK }\end{array}$ & $\begin{array}{l}\text { TEXAS UTILITIES } \\
\text { ELEC CO }\end{array}$ & $\mathrm{TX}$ & 44372 & 3453 & 7 & 136 & Operational & 1958 & GAS & FRESH & ONCE THROUGH \\
\hline MUSCATINE & $\begin{array}{l}\text { MUSCATINE } \\
\text { POWER \& WATER }\end{array}$ & IA & 13143 & 1167 & 7 & 22.5 & Operational & 1958 & COAL & FRESH & ONCE THROUGH \\
\hline MUSCATINE & $\begin{array}{l}\text { MUSCATINE } \\
\text { POWER \& WATER }\end{array}$ & $\mathrm{IA}$ & 13143 & 1167 & 8 & 66 & Operational & 1969 & COAL & FRESH & ONCE THROUGH \\
\hline MUSCATINE & $\begin{array}{l}\text { MUSCATINE } \\
\text { POWER \& WATER }\end{array}$ & IA & 13143 & 1167 & 9 & 160 & Operational & 1983 & COAL & FRESH & ONCE THROUGH \\
\hline $\begin{array}{l}\text { MUSKINGUM } \\
\text { RIVER }\end{array}$ & OHIO POWER CO & $\mathrm{OH}$ & 14006 & 2872 & 3 & 238 & Operational & 1957 & COAL & FRESH & ONCE THROUGH \\
\hline $\begin{array}{l}\text { MUSKINGUM } \\
\text { RIVER }\end{array}$ & OHIO POWER CO & $\mathrm{OH}$ & 14006 & 2872 & 4 & 238 & Operational & 1958 & COAL & FRESH & ONCE THROUGH \\
\hline $\begin{array}{l}\text { MUSKINGUM } \\
\text { RIVER }\end{array}$ & OHIO POWER CO & $\mathrm{OH}$ & 14006 & 2872 & 2 & 220 & Operational & 1954 & COAL & FRESH & ONCE THROUGH \\
\hline $\begin{array}{l}\text { MUSKINGUM } \\
\text { RIVER }\end{array}$ & OHIO POWER CO & $\mathrm{OH}$ & 14006 & 2872 & 1 & 220 & Operational & 1953 & COAL & FRESH & ONCE THROUGH \\
\hline MUSKOGEE & $\begin{array}{l}\text { OKLAHOMA GAS \& } \\
\text { ELEC CO }\end{array}$ & OK & 14063 & 2952 & 3 & 173 & Operational & 1956 & GAS & FRESH & ONCE THROUGH \\
\hline MYSTIC & BOSTON EDISON CO & MA & 1998 & 1588 & 4 & 156 & Operational & 1957 & OIL & BRACKISH & ONCE THROUGH \\
\hline MYSTIC & BOSTON EDISON CO & MA & 1998 & 1588 & 5 & 156 & Operational & 1959 & OIL & BRACKISH & ONCE THROUGH \\
\hline MYSTIC & BOSTON EDISON CO & MA & 1998 & 1588 & 6 & 156 & Operational & 1961 & OIL & BRACKISH & ONCE THROUGH \\
\hline MYSTIC & BOSTON EDISON CO & MA & 1998 & 1588 & 7 & 617 & Operational & 1975 & OIL & BRACKISH & ONCE THROUGH \\
\hline $\begin{array}{l}\text { NEARMAN } \\
\text { CREEK }\end{array}$ & $\begin{array}{l}\text { KANSAS CITY BD } \\
\text { PUB UTIL }\end{array}$ & KS & 9996 & 6064 & 1 & 261 & Operational & 1981 & COAL & FRESH & ONCE THROUGH \\
\hline
\end{tabular}




\begin{tabular}{|c|c|c|c|c|c|c|c|c|c|c|c|}
\hline Plant Name & Utility Name & State & Utility/P & $n t / U n$ & $I D$ & $M W$ & Status & Year & Fuel & Water & Cooling System \\
\hline $\begin{array}{l}\text { NEBRASKA } \\
\text { CITY }\end{array}$ & $\begin{array}{l}\text { OMAHA PUBLIC } \\
\text { POWER DIST }\end{array}$ & $\mathrm{NE}$ & 14127 & 6096 & 1 & 616 & Operational & 1979 & COAL & FRESH & ONCE THROUGH \\
\hline $\begin{array}{l}\text { NELSON } \\
\text { DEWEY }\end{array}$ & $\begin{array}{l}\text { WISCONSIN } \\
\text { POWER \& LIGHT }\end{array}$ & WI & 20856 & 4054 & 2 & 114 & Operational & 1962 & COAL & FRESH & ONCE THROUGH \\
\hline $\begin{array}{l}\text { NELSON } \\
\text { DEWEY }\end{array}$ & $\begin{array}{l}\text { WISCONSIN } \\
\text { POWER \& LIGHT }\end{array}$ & WI & 20856 & 4054 & 1 & 114 & Operational & 1959 & COAL & FRESH & ONCE THROUGH \\
\hline NEW BOSTON & BOSTON EDISON CO & MA & 1998 & 1589 & 1 & 359 & Operational & 1965 & GAS & SALINE & ONCE THROUGH \\
\hline NEW BOSTON & BOSTON EDISON CO & MA & 1998 & 1589 & 2 & 359 & Operational & 1967 & GAS & SALINE & ONCE THROUGH \\
\hline NEW CASTLE & $\begin{array}{l}\text { PENNSYLVANIA } \\
\text { POWER CO }\end{array}$ & PA & 14716 & 3138 & 3 & 97.8 & Operational & 1952 & COAL & FRESH & ONCE THROUGH \\
\hline NEW CASTLE & $\begin{array}{l}\text { PENNSYLVANIA } \\
\text { POWER CO }\end{array}$ & PA & 14716 & 3138 & 4 & 114 & Operational & 1958 & COAL & FRESH & ONCE THROUGH \\
\hline NEW CASTLE & $\begin{array}{l}\text { PENNSYLVANIA } \\
\text { POWER CO }\end{array}$ & PA & 14716 & 3138 & 5 & 136 & Operational & 1964 & COAL & FRESH & ONCE THROUGH \\
\hline $\begin{array}{l}\text { NEW HAVEN } \\
\text { HARBOR }\end{array}$ & $\begin{array}{l}\text { UNITED } \\
\text { ILLUMINATING CO }\end{array}$ & CT & 19497 & 6156 & 1 & 460 & Operational & 1975 & OIL & SALINE & ONCE THROUGH \\
\hline NEW MADRID & $\begin{array}{l}\text { ASSOCIATED } \\
\text { ELECTRIC COOP }\end{array}$ & MO & 924 & 2167 & 1 & 600 & Operational & 1972 & COAL & FRESH & ONCE THROUGH \\
\hline NEW MADRID & $\begin{array}{l}\text { ASSOCIATED } \\
\text { ELECTRIC COOP }\end{array}$ & MO & 924 & 2167 & 2 & 600 & Operational & 1977 & COAL & FRESH & ONCE THROUGH \\
\hline NEWINGTON & $\begin{array}{l}\text { PUBLIC SERV NEW } \\
\text { HAMPSHIRE }\end{array}$ & $\mathrm{NH}$ & 15472 & 8002 & 1 & 414 & Operational & 1974 & OIL & BRACKISH & ONCE THROUGH \\
\hline $\operatorname{NILES}(\mathrm{OH})$ & OHIO EDISON CO & $\mathrm{OH}$ & 13998 & 2861 & 1 & 125 & Operational & 1954 & COAL & FRESH & ONCE THROUGH \\
\hline NILES $(\mathrm{OH})$ & OHIO EDISON CO & $\mathrm{OH}$ & 13998 & 2861 & 2 & 125 & Operational & 1954 & COAL & FRESH & ONCE THROUGH \\
\hline
\end{tabular}




\begin{tabular}{|c|c|c|c|c|c|c|c|c|c|c|c|}
\hline Plant Name & Utility Name & State & Utility/Pl & $n t / U n i$ & $I D$ & $M W$ & Status & Year & Fuel & Water & Cooling System \\
\hline $\begin{array}{l}\text { NINE MILE } \\
\text { POINT }\end{array}$ & $\begin{array}{l}\text { NIAGARA MOHAWK } \\
\text { POWER CORP }\end{array}$ & NY & 13573 & 2589 & 1 & 642 & Operational & 1969 & UR & FRESH & ONCE THROUGH \\
\hline $\begin{array}{l}\text { NINEMILE } \\
\text { POINT }\end{array}$ & $\begin{array}{l}\text { LOUISIANA POWER } \\
\& \text { LIGHT }\end{array}$ & LA & 11241 & 1403 & 4 & 783 & Operational & 1971 & GAS & FRESH & ONCE THROUGH \\
\hline $\begin{array}{l}\text { NINEMILE } \\
\text { POINT }\end{array}$ & $\begin{array}{l}\text { LOUISIANA POWER } \\
\& \text { LIGHT }\end{array}$ & LA & 11241 & 1403 & 5 & 783 & Operational & 1973 & GAS & FRESH & ONCE THROUGH \\
\hline $\begin{array}{l}\text { NINEMILE } \\
\text { POINT }\end{array}$ & $\begin{array}{l}\text { LOUISIANA POWER } \\
\text { \& LIGHT }\end{array}$ & LA & 11241 & 1403 & 2 & 103 & Operational & 1953 & GAS & FRESH & ONCE THROUGH \\
\hline $\begin{array}{l}\text { NINEMILE } \\
\text { POINT }\end{array}$ & $\begin{array}{l}\text { LOUISIANA POWER } \\
\& \text { LIGHT }\end{array}$ & LA & 11241 & 1403 & 1 & 57 & Operational & 1951 & GAS & FRESH & ONCE THROUGH \\
\hline $\begin{array}{l}\text { NINEMILE } \\
\text { POINT }\end{array}$ & $\begin{array}{l}\text { LOUISIANA POWER } \\
\& \text { LIGHT }\end{array}$ & LA & 11241 & 1403 & 3 & 136 & Operational & 1955 & GAS & FRESH & ONCE THROUGH \\
\hline NOBLESVILLE & PSI ENERGY INC & IN & 15470 & 1007 & 1 & 50 & Operational & 1950 & COAL & FRESH & $\begin{array}{l}\text { COMBINATION (ONCE } \\
\text { THROUGH/HELPER TOWER) }\end{array}$ \\
\hline NOBLESVILLE & PSI ENERGY INC & IN & 15470 & 1007 & 2 & 50 & Operational & 1950 & COAL & FRESH & $\begin{array}{l}\text { COMBINATION (ONCE } \\
\text { THROUGH/HELPER TOWER) }\end{array}$ \\
\hline $\begin{array}{l}\text { NORTH } \\
\text { DENVER }\end{array}$ & $\begin{array}{l}\text { HASTINGS } \\
\text { UTILITIES DEPT }\end{array}$ & NE & 8245 & 60 & 4 & 17 & Standby & 1957 & GAS & GROUND & ONCE THROUGH \\
\hline NORTH LAKE & $\begin{array}{l}\text { TEXAS UTILITIES } \\
\text { ELEC CO }\end{array}$ & $\mathrm{TX}$ & 44372 & 3454 & 1 & 177 & Operational & 1959 & GAS & FRESH & ONCE THROUGH \\
\hline NORTH LAKE & $\begin{array}{l}\text { TEXAS UTILITIES } \\
\text { ELEC CO }\end{array}$ & TX & 44372 & 3454 & 2 & 171 & Operational & 1961 & GAS & FRESH & ONCE THROUGH \\
\hline NORTH LAKE & $\begin{array}{l}\text { TEXAS UTILITIES } \\
\text { ELEC CO }\end{array}$ & $\mathrm{TX}$ & 44372 & 3454 & 3 & 361 & Operational & 1964 & GAS & FRESH & ONCE THROUGH \\
\hline NORTH OMAHA & $\begin{array}{l}\text { OMAHA PUBLIC } \\
\text { POWER DIST }\end{array}$ & NE & 14127 & 2291 & 1 & 73.5 & Operational & 1954 & COAL & FRESH & ONCE THROUGH \\
\hline NORTH OMAHA & $\begin{array}{l}\text { OMAHA PUBLIC } \\
\text { POWER DIST }\end{array}$ & NE & 14127 & 2291 & 2 & 109 & Operational & 1957 & COAL & FRESH & ONCE THROUGH \\
\hline
\end{tabular}




\begin{tabular}{|c|c|c|c|c|c|c|c|c|c|c|c|}
\hline Plant Name & Utility Name & State & Utility/P & $n t / U n$ & $I D$ & $M W$ & Status & Year & Fuel & Water & Cooling System \\
\hline NORTH OMAHA & $\begin{array}{l}\text { OMAHA PUBLIC } \\
\text { POWER DIST }\end{array}$ & NE & 14127 & 2291 & 3 & 109 & Operational & 1959 & COAL & FRESH & ONCE THROUGH \\
\hline NORTH OMAHA & $\begin{array}{l}\text { OMAHA PUBLIC } \\
\text { POWER DIST }\end{array}$ & NE & 14127 & 2291 & 4 & 136 & Operational & 1963 & COAL & FRESH & ONCE THROUGH \\
\hline NORTH OMAHA & $\begin{array}{l}\text { OMAHA PUBLIC } \\
\text { POWER DIST }\end{array}$ & NE & 14127 & 2291 & 5 & 218 & Operational & 1968 & COAL & FRESH & ONCE THROUGH \\
\hline NORTH TEXAS & $\begin{array}{l}\text { BRAZOS ELEC } \\
\text { POWER COOP }\end{array}$ & $\mathrm{TX}$ & 2172 & 3627 & 3 & 38 & Operational & 1963 & GAS & FRESH & ONCE THROUGH \\
\hline NORTH TEXAS & $\begin{array}{l}\text { BRAZOS ELEC } \\
\text { POWER COOP }\end{array}$ & TX & 2172 & 3627 & 2 & 16.5 & Operational & 1958 & GAS & FRESH & ONCE THROUGH \\
\hline NORTH TEXAS & $\begin{array}{l}\text { BRAZOS ELEC } \\
\text { POWER COOP }\end{array}$ & $\mathrm{TX}$ & 2172 & 3627 & 1 & 16.5 & Operational & 1958 & GAS & FRESH & ONCE THROUGH \\
\hline NORTHPORT & $\begin{array}{l}\text { LONG ISLAND } \\
\text { LIGHTING CO }\end{array}$ & NY & 11172 & 2516 & 1 & 387 & Operational & 1967 & OIL & SALINE & ONCE THROUGH \\
\hline NORTHPORT & $\begin{array}{l}\text { LONG ISLAND } \\
\text { LIGHTING CO }\end{array}$ & NY & 11172 & 2516 & 4 & 387 & Operational & 1977 & GAS & SALINE & ONCE THROUGH \\
\hline NORTHPORT & $\begin{array}{l}\text { LONG ISLAND } \\
\text { LIGHTING CO }\end{array}$ & NY & 11172 & 2516 & 2 & 387 & Operational & 1968 & GAS & SALINE & ONCE THROUGH \\
\hline NORTHPORT & $\begin{array}{l}\text { LONG ISLAND } \\
\text { LIGHTING CO }\end{array}$ & NY & 11172 & 2516 & 3 & 387 & Operational & 1972 & OIL & SALINE & ONCE THROUGH \\
\hline NORTHSIDE & $\begin{array}{l}\text { JACKSONVILLE } \\
\text { ELEC AUTH }\end{array}$ & $\mathrm{FL}$ & 9617 & 667 & 1 & 298 & Operational & 1966 & OIL & BRACKISH & ONCE THROUGH \\
\hline NORTHSIDE & $\begin{array}{l}\text { JACKSONVILLE } \\
\text { ELEC AUTH }\end{array}$ & $\mathrm{FL}$ & 9617 & 667 & 2 & 298 & Operational & 1972 & OIL & BRACKISH & ONCE THROUGH \\
\hline NORTHSIDE & $\begin{array}{l}\text { JACKSONVILLE } \\
\text { ELEC AUTH }\end{array}$ & $\mathrm{FL}$ & 9617 & 667 & 3 & 564 & Operational & 1977 & OIL & BRACKISH & ONCE THROUGH \\
\hline $\begin{array}{l}\text { NORWALK } \\
\text { HARBOR }\end{array}$ & $\begin{array}{l}\text { CONNECTICUT } \\
\text { LIGHT \& POWER }\end{array}$ & CT & 4176 & 548 & 2 & 163 & Operational & 1963 & OIL & SALINE & ONCE THROUGH \\
\hline
\end{tabular}




\begin{tabular}{|c|c|c|c|c|c|c|c|c|c|c|c|}
\hline Plant Name & Utility Name & State & Utility/Pl & $n t / U n i$ & $I D$ & $\boldsymbol{M W}$ & Status & Year & Fuel & Water & Cooling System \\
\hline $\begin{array}{l}\text { NORWALK } \\
\text { HARBOR }\end{array}$ & $\begin{array}{l}\text { CONNECTICUT } \\
\text { LIGHT \& POWER }\end{array}$ & CT & 4176 & 548 & 1 & 163 & Operational & 1960 & OIL & SALINE & ONCE THROUGH \\
\hline NUECES BAY & $\begin{array}{l}\text { CENTRAL POWER \& } \\
\text { LIGHT CO }\end{array}$ & TX & 3278 & 3441 & 5 & 34.5 & Standby & 1949 & GAS & BRACKISH & ONCE THROUGH \\
\hline NUECES BAY & $\begin{array}{l}\text { CENTRAL POWER \& } \\
\text { LIGHT CO }\end{array}$ & TX & 3278 & 3441 & 6 & 180 & Operational & 1965 & GAS & BRACKISH & ONCE THROUGH \\
\hline NUECES BAY & $\begin{array}{l}\text { CENTRAL POWER \& } \\
\text { LIGHT CO }\end{array}$ & TX & 3278 & 3441 & 7 & 351 & Operational & 1972 & GAS & BRACKISH & ONCE THROUGH \\
\hline $\begin{array}{l}\text { OAK CREEK } \\
\text { (TX) }\end{array}$ & $\begin{array}{l}\text { WEST TEXAS UTIL } \\
\text { CO }\end{array}$ & $\mathrm{TX}$ & 20404 & 3523 & 1 & 81.6 & Operational & 1962 & GAS & FRESH & ONCE THROUGH \\
\hline $\begin{array}{l}\text { OAK CREEK } \\
\text { (WI) }\end{array}$ & $\begin{array}{l}\text { WISCONSIN ELEC } \\
\text { POWER CO }\end{array}$ & WI & 20847 & 4041 & 6 & 275 & Operational & 1961 & COAL & FRESH & ONCE THROUGH \\
\hline $\begin{array}{l}\text { OAK CREEK } \\
\text { (WI) }\end{array}$ & $\begin{array}{l}\text { WISCONSIN ELEC } \\
\text { POWER CO }\end{array}$ & WI & 20847 & 4041 & 7 & 318 & Operational & 1965 & COAL & FRESH & ONCE THROUGH \\
\hline $\begin{array}{l}\text { OAK CREEK } \\
\text { (WI) }\end{array}$ & $\begin{array}{l}\text { WISCONSIN ELEC } \\
\text { POWER CO }\end{array}$ & WI & 20847 & 4041 & 5 & 275 & Operational & 1959 & COAL & FRESH & ONCE THROUGH \\
\hline $\begin{array}{l}\text { OAK CREEK } \\
\text { (WI) }\end{array}$ & $\begin{array}{l}\text { WISCONSIN ELEC } \\
\text { POWER CO }\end{array}$ & WI & 20847 & 4041 & 8 & 324 & Operational & 1967 & COAL & FRESH & ONCE THROUGH \\
\hline OCONEE & DUKE POWER CO & SC & 5416 & 3265 & 1 & 925 & Operational & 1973 & UR & FRESH & ONCE THROUGH \\
\hline OCONEE & DUKE POWER CO & SC & 5416 & 3265 & 2 & 925 & Operational & 1974 & UR & FRESH & ONCE THROUGH \\
\hline OCONEE & DUKE POWER CO & SC & 5416 & 3265 & 3 & 925 & Operational & 1974 & UR & FRESH & ONCE THROUGH \\
\hline $\begin{array}{l}\text { ORMOND } \\
\text { BEACH }\end{array}$ & $\begin{array}{l}\text { SOUTHERN CALIF } \\
\text { EDISON CO }\end{array}$ & $\mathrm{CA}$ & 17609 & 350 & 1 & 806 & Operational & 1971 & GAS & SALINE & ONCE THROUGH \\
\hline $\begin{array}{l}\text { ORMOND } \\
\text { BEACH }\end{array}$ & $\begin{array}{l}\text { SOUTHERN CALIF } \\
\text { EDISON CO }\end{array}$ & $\mathrm{CA}$ & 17609 & 350 & 2 & 806 & Operational & 1973 & GAS & SALINE & ONCE THROUGH \\
\hline
\end{tabular}




\begin{tabular}{|c|c|c|c|c|c|c|c|c|c|c|c|}
\hline Plant Name & Utility Name & State & Utility/Pl & $n t / U n i$ & $I D$ & $\boldsymbol{M W}$ & Status & Year & Fuel & Water & Cooling System \\
\hline OSWEGO & $\begin{array}{l}\text { NIAGARA MOHAWK } \\
\text { POWER CORP }\end{array}$ & NY & 13573 & 2594 & 3 & 92 & Operational & 1948 & GAS & FRESH & ONCE THROUGH \\
\hline OSWEGO & $\begin{array}{l}\text { NIAGARA MOHAWK } \\
\text { POWER CORP }\end{array}$ & NY & 13573 & 2594 & 6 & 902 & Operational & 1980 & OIL & FRESH & ONCE THROUGH \\
\hline OSWEGO & $\begin{array}{l}\text { NIAGARA MOHAWK } \\
\text { POWER CORP }\end{array}$ & NY & 13573 & 2594 & 4 & 100 & Operational & 1951 & OIL & FRESH & ONCE THROUGH \\
\hline OSWEGO & $\begin{array}{l}\text { NIAGARA MOHAWK } \\
\text { POWER CORP }\end{array}$ & NY & 13573 & 2594 & 5 & 902 & Standby & 1975 & OIL & FRESH & ONCE THROUGH \\
\hline $\begin{array}{l}\text { OYSTER } \\
\text { CREEK }\end{array}$ & $\begin{array}{l}\text { GPU NUCLEAR } \\
\text { CORP }\end{array}$ & NJ & 7423 & 2388 & 1 & 550 & Operational & 1969 & UR & BRACKISH & ONCE THROUGH \\
\hline PAINT CREEK & $\begin{array}{l}\text { WEST TEXAS UTIL } \\
\text { CO }\end{array}$ & TX & 20404 & 3524 & 1 & 34.5 & Operational & 1953 & GAS & FRESH & ONCE THROUGH \\
\hline PAINT CREEK & $\begin{array}{l}\text { WEST TEXAS UTIL } \\
\text { CO }\end{array}$ & $\mathrm{TX}$ & 20404 & 3524 & 2 & 37.5 & Operational & 1954 & GAS & FRESH & ONCE THROUGH \\
\hline PAINT CREEK & $\begin{array}{l}\text { WEST TEXAS UTIL } \\
\text { CO }\end{array}$ & $\mathrm{TX}$ & 20404 & 3524 & 3 & 54.4 & Operational & 1959 & GAS & FRESH & ONCE THROUGH \\
\hline PAINT CREEK & $\begin{array}{l}\text { WEST TEXAS UTIL } \\
\text { CO }\end{array}$ & TX & 20404 & 3524 & 4 & 115 & Operational & 1971 & GAS & FRESH & ONCE THROUGH \\
\hline PALISADES (MI) & $\begin{array}{l}\text { CONSUMERS } \\
\text { POWER CO }\end{array}$ & $\mathrm{Ml}$ & 4254 & 1715 & 1 & 812 & Operational & 1971 & UR & FRESH & $\begin{array}{l}\text { COMBINATION (ONCE } \\
\text { THROUGH/HELPER TOWER) }\end{array}$ \\
\hline PALO SECO & $\begin{array}{l}\text { PUERTO RICO ELEC } \\
\text { PWR AUTH }\end{array}$ & PR & 15497 & & 2 & 93.8 & Operational & 1961 & OIL & SALINE & ONCE THROUGH \\
\hline PALO SECO & $\begin{array}{l}\text { PUERTO RICO ELEC } \\
\text { PWR AUTH }\end{array}$ & PR & 15497 & & 3 & 229 & Operational & 1970 & OIL & SALINE & ONCE THROUGH \\
\hline PALO SECO & $\begin{array}{l}\text { PUERTO RICO ELEC } \\
\text { PWR AUTH }\end{array}$ & PR & 15497 & & 1 & 93.8 & Operational & 1960 & OIL & SALINE & ONCE THROUGH \\
\hline PALO SECO & $\begin{array}{l}\text { PUERTO RICO ELEC } \\
\text { PWR AUTH }\end{array}$ & PR & 15497 & & 4 & 229 & Operational & 1970 & OIL & SALINE & ONCE THROUGH \\
\hline
\end{tabular}




\begin{tabular}{|c|c|c|c|c|c|c|c|c|c|c|c|}
\hline Plant Name & Utility Name & State & Utility/Pl & $n t / U n i$ & $I D$ & $M W$ & Status & Year & Fuel & Water & Cooling System \\
\hline PARADISE & $\begin{array}{l}\text { TENNESSEE } \\
\text { VALLEY AUTH }\end{array}$ & KY & 18642 & 1378 & 1 & 704 & Operational & 1963 & COAL & FRESH & $\begin{array}{l}\text { MIXED MODE (ONCE } \\
\text { THROUGH/COOLING TOWER) }\end{array}$ \\
\hline PARADISE & $\begin{array}{l}\text { TENNESSEE } \\
\text { VALLEY AUTH }\end{array}$ & KY & 18642 & 1378 & 2 & 704 & Operational & 1963 & COAL & FRESH & $\begin{array}{l}\text { MIXED MODE (ONCE } \\
\text { THROUGH/COOLING TOWER) }\end{array}$ \\
\hline PATERSON & $\begin{array}{l}\text { NEW ORLEANS } \\
\text { PUBLIC SERV }\end{array}$ & LA & 13478 & 1407 & 3 & 51.8 & Standby & 1950 & GAS & BRACKISH & ONCE THROUGH \\
\hline PATERSON & $\begin{array}{l}\text { NEW ORLEANS } \\
\text { PUBLIC SERV }\end{array}$ & LA & 13478 & 1407 & 4 & 81.3 & Standby & 1954 & GAS & BRACKISH & ONCE THROUGH \\
\hline $\begin{array}{l}\text { PEACH } \\
\text { BOTTOM }\end{array}$ & PECO ENERGY CO & PA & 14940 & 3166 & 3 & 1152 & Operational & 1974 & UR & FRESH & $\begin{array}{l}\text { COMBINATION (ONCE } \\
\text { THROUGH/HELPER TOWER) }\end{array}$ \\
\hline $\begin{array}{l}\text { PEACH } \\
\text { BOTTOM }\end{array}$ & PECO ENERGY CO & PA & 14940 & 3166 & 2 & 1152 & Operational & 1974 & UR & FRESH & $\begin{array}{l}\text { COMBINATION (ONCE } \\
\text { THROUGH/HELPER TOWER) }\end{array}$ \\
\hline PEARL & $\begin{array}{l}\text { SOYLAND POWER } \\
\text { COOP }\end{array}$ & $\mathrm{IL}$ & 40307 & 6238 & 1 & 22 & Operational & 1966 & COAL & FRESH & ONCE THROUGH \\
\hline PERRY (IN) & $\begin{array}{l}\text { INDIANAPOLIS } \\
\text { POWER \& LT }\end{array}$ & IN & 9273 & 992 & 7 & 11.6 & Operational & 1966 & COAL & FRESH & $\begin{array}{l}\text { COMBINATION (ONCE } \\
\text { THROUGH/SPRAY TOWER) }\end{array}$ \\
\hline PERRY (IN) & $\begin{array}{l}\text { INDIANAPOLIS } \\
\text { POWER \& LT }\end{array}$ & IN & 9273 & 992 & 4 & 15 & Operational & 1925 & COAL & FRESH & $\begin{array}{l}\text { COMBINATION (ONCE } \\
\text { THROUGH/SPRAY TOWER) }\end{array}$ \\
\hline PERU (IL) & $\begin{array}{l}\text { PERU (IL) LIGHT } \\
\text { DEPT }\end{array}$ & $\mathrm{IL}$ & 14840 & 3166 & 4 & 7.5 & Standby & 1960 & GAS & FRESH & ONCE THROUGH \\
\hline PERU (IN) & PERU (IN) UTILITIES & IN & 14839 & 1037 & 2 & 22 & Operational & 1948 & COAL & FRESH & ONCE THROUGH \\
\hline PERU (IN) & PERU (IN) UTILITIES & IN & 14839 & 1037 & 3 & 10 & Operational & 1959 & COAL & FRESH & ONCE THROUGH \\
\hline PETERSBURG & $\begin{array}{l}\text { INDIANAPOLIS } \\
\text { POWER \& LT }\end{array}$ & IN & 9273 & 994 & 2 & 471 & Operational & 1969 & COAL & FRESH & $\begin{array}{l}\text { COMBINATION (ONCE } \\
\text { THROUGH/HELPER TOWER) }\end{array}$ \\
\hline PETERSBURG & $\begin{array}{l}\text { INDIANAPOLIS } \\
\text { POWER \& LT }\end{array}$ & IN & 9273 & 994 & 1 & 253 & Operational & 1967 & COAL & FRESH & ONCE THROUGH \\
\hline
\end{tabular}




\begin{tabular}{|c|c|c|c|c|c|c|c|c|c|c|c|}
\hline Plant Name & Utility Name & State & Utility/Pl & $n t / U n i$ & $I D$ & $\boldsymbol{M W}$ & Status & Year & Fuel & Water & Cooling System \\
\hline PH ROBINSON & $\begin{array}{l}\text { HOUSTON } \\
\text { LIGHTING \& POWER }\end{array}$ & $\mathrm{TX}$ & 8901 & 3466 & 1 & 485 & Operational & 1966 & GAS & BRACKISH & $\begin{array}{l}\text { COMBINATION (ONCE } \\
\text { THROUGH/HELPER TOWER) }\end{array}$ \\
\hline PH ROBINSON & $\begin{array}{l}\text { HOUSTON } \\
\text { LIGHTING \& POWER }\end{array}$ & $\mathrm{TX}$ & 8901 & 3466 & 2 & 485 & Operational & 1967 & GAS & BRACKISH & $\begin{array}{l}\text { COMBINATION (ONCE } \\
\text { THROUGH/HELPER TOWER) }\end{array}$ \\
\hline PH ROBINSON & $\begin{array}{l}\text { HOUSTON } \\
\text { LIGHTING \& POWER }\end{array}$ & $\mathrm{TX}$ & 8901 & 3466 & 3 & 581 & Operational & 1968 & GAS & BRACKISH & $\begin{array}{l}\text { COMBINATION (ONCE } \\
\text { THROUGH/HELPER TOWER) }\end{array}$ \\
\hline PH ROBINSON & $\begin{array}{l}\text { HOUSTON } \\
\text { LIGHTING \& POWER }\end{array}$ & $\mathrm{TX}$ & 8901 & 3466 & 4 & 765 & Operational & 1973 & GAS & BRACKISH & $\begin{array}{l}\text { COMBINATION (ONCE } \\
\text { THROUGH/HELPER TOWER) }\end{array}$ \\
\hline PHILIP SPORN & $\begin{array}{l}\text { CENTRAL } \\
\text { OPERATING CO }\end{array}$ & WV & 3277 & 3938 & 4 & 153 & Operational & 1952 & COAL & FRESH & ONCE THROUGH \\
\hline PHILIP SPORN & $\begin{array}{l}\text { CENTRAL } \\
\text { OPERATING CO }\end{array}$ & WV & 3277 & 3938 & 3 & 153 & Operational & 1951 & COAL & FRESH & ONCE THROUGH \\
\hline PHILIP SPORN & $\begin{array}{l}\text { CENTRAL } \\
\text { OPERATING CO }\end{array}$ & WV & 3277 & 3938 & 2 & 153 & Operational & 1950 & COAL & FRESH & ONCE THROUGH \\
\hline PHILIP SPORN & $\begin{array}{l}\text { CENTRAL } \\
\text { OPERATING CO }\end{array}$ & WV & 3277 & 3938 & 1 & 153 & Operational & 1950 & COAL & FRESH & ONCE THROUGH \\
\hline PHILIP SPORN & $\begin{array}{l}\text { CENTRAL } \\
\text { OPERATING CO }\end{array}$ & WV & 3277 & 3938 & 5 & 496 & Operational & 1960 & COAL & FRESH & ONCE THROUGH \\
\hline PICWAY & $\begin{array}{l}\text { COLUMBUS } \\
\text { SOUTHERN POWER }\end{array}$ & $\mathrm{OH}$ & 4062 & 2843 & 5 & 106 & Operational & 1955 & COAL & FRESH & ONCE THROUGH \\
\hline PILGRIM & BOSTON EDISON CO & MA & 1998 & 1590 & 1 & 678 & Operational & 1972 & UR & SALINE & ONCE THROUGH \\
\hline PIQUA & $\begin{array}{l}\text { PIQUA MUNI } \\
\text { POWER SYSTEM }\end{array}$ & $\mathrm{OH}$ & 15095 & 2937 & 6 & 12.5 & Operational & 1951 & COAL & FRESH & ONCE THROUGH \\
\hline PIQUA & $\begin{array}{l}\text { PIQUA MUNI } \\
\text { POWER SYSTEM }\end{array}$ & $\mathrm{OH}$ & 15095 & 2937 & 7 & 20 & Operational & 1961 & COAL & FRESH & ONCE THROUGH \\
\hline PIQUA & $\begin{array}{l}\text { PIQUA MUNI } \\
\text { POWER SYSTEM }\end{array}$ & $\mathrm{OH}$ & 15095 & 2937 & 4 & 7.5 & Operational & 1947 & COAL & FRESH & ONCE THROUGH \\
\hline
\end{tabular}




\begin{tabular}{|c|c|c|c|c|c|c|c|c|c|c|c|}
\hline Plant Name & Utility Name & State & Utility/Pl & $n t / U n i$ & $I D$ & $M W$ & Status & Year & Fuel & Water & Cooling System \\
\hline PITTSBURG & $\begin{array}{l}\text { PACIFIC GAS \& } \\
\text { ELEC CO }\end{array}$ & $\mathrm{CA}$ & 14328 & 271 & 4 & 156 & Operational & 1954 & GAS & BRACKISH & ONCE THROUGH \\
\hline PITTSBURG & $\begin{array}{l}\text { PACIFIC GAS \& } \\
\text { ELEC CO }\end{array}$ & $\mathrm{CA}$ & 14328 & 271 & 5 & 326 & Operational & 1960 & GAS & BRACKISH & ONCE THROUGH \\
\hline PITTSBURG & $\begin{array}{l}\text { PACIFIC GAS \& } \\
\text { ELEC CO }\end{array}$ & $\mathrm{CA}$ & 14328 & 271 & 6 & 326 & Operational & 1961 & GAS & BRACKISH & ONCE THROUGH \\
\hline PITTSBURG & $\begin{array}{l}\text { PACIFIC GAS \& } \\
\text { ELEC CO }\end{array}$ & $\mathrm{CA}$ & 14328 & 271 & 2 & 156 & Operational & 1954 & GAS & BRACKISH & ONCE THROUGH \\
\hline PITTSBURG & $\begin{array}{l}\text { PACIFIC GAS \& } \\
\text { ELEC CO }\end{array}$ & $\mathrm{CA}$ & 14328 & 271 & 1 & 156 & Operational & 1954 & GAS & BRACKISH & ONCE THROUGH \\
\hline PITTSBURG & $\begin{array}{l}\text { PACIFIC GAS \& } \\
\text { ELEC CO }\end{array}$ & $\mathrm{CA}$ & 14328 & 271 & 3 & 156 & Operational & 1954 & GAS & BRACKISH & ONCE THROUGH \\
\hline POINT BEACH & $\begin{array}{l}\text { WISCONSIN ELEC } \\
\text { POWER CO }\end{array}$ & WI & 20847 & 4046 & 1 & 524 & Operational & 1970 & UR & FRESH & ONCE THROUGH \\
\hline POINT BEACH & $\begin{array}{l}\text { WISCONSIN ELEC } \\
\text { POWER CO }\end{array}$ & WI & 20847 & 4046 & 2 & 524 & Operational & 1972 & UR & FRESH & ONCE THROUGH \\
\hline PORT ALLEN & $\begin{array}{l}\text { CITIZENS UTILITIES } \\
(\mathrm{HI})\end{array}$ & $\mathrm{HI}$ & 3611 & 6474 & 1 & 10 & Operational & 1968 & OIL & SALINE & ONCE THROUGH \\
\hline $\begin{array}{l}\text { PORT } \\
\text { EVERGLADES }\end{array}$ & $\begin{array}{l}\text { FLORIDA POWER \& } \\
\text { LIGHT CO }\end{array}$ & $\mathrm{FL}$ & 6452 & 617 & 1 & 225 & Operational & 1960 & OIL & SALINE & ONCE THROUGH \\
\hline $\begin{array}{l}\text { PORT } \\
\text { EVERGLADES }\end{array}$ & $\begin{array}{l}\text { FLORIDA POWER \& } \\
\text { LIGHT CO }\end{array}$ & $\mathrm{FL}$ & 6452 & 617 & 4 & 402 & Operational & 1965 & OIL & SALINE & ONCE THROUGH \\
\hline $\begin{array}{l}\text { PORT } \\
\text { EVERGLADES }\end{array}$ & $\begin{array}{l}\text { FLORIDA POWER \& } \\
\text { LIGHT CO }\end{array}$ & $\mathrm{FL}$ & 6452 & 617 & 2 & 225 & Operational & 1961 & OIL & SALINE & ONCE THROUGH \\
\hline $\begin{array}{l}\text { PORT } \\
\text { EVERGLADES }\end{array}$ & $\begin{array}{l}\text { FLORIDA POWER \& } \\
\text { LIGHT CO }\end{array}$ & $\mathrm{FL}$ & 6452 & 617 & 3 & 402 & Operational & 1964 & OIL & SALINE & ONCE THROUGH \\
\hline $\begin{array}{l}\text { PORT } \\
\text { JEFFERSON }\end{array}$ & $\begin{array}{l}\text { LONG ISLAND } \\
\text { LIGHTING CO }\end{array}$ & NY & 11172 & 2517 & 1 & 46 & Operational & 1948 & OIL & SALINE & ONCE THROUGH \\
\hline
\end{tabular}




\begin{tabular}{|c|c|c|c|c|c|c|c|c|c|c|c|}
\hline Plant Name & Utility Name & State & Utility/Pl & nt/Uni & $I D$ & $M W$ & Status & Year & Fuel & Water & Cooling System \\
\hline $\begin{array}{l}\text { PORT } \\
\text { JEFFERSON }\end{array}$ & $\begin{array}{l}\text { LONG ISLAND } \\
\text { LIGHTING CO }\end{array}$ & NY & 11172 & 2517 & 2 & 46 & Operational & 1950 & OIL & SALINE & ONCE THROUGH \\
\hline $\begin{array}{l}\text { PORT } \\
\text { JEFFERSON }\end{array}$ & $\begin{array}{l}\text { LONG ISLAND } \\
\text { LIGHTING CO }\end{array}$ & NY & 11172 & 2517 & 3 & 188 & Operational & 1958 & OIL & SALINE & ONCE THROUGH \\
\hline $\begin{array}{l}\text { PORT } \\
\text { JEFFERSON }\end{array}$ & $\begin{array}{l}\text { LONG ISLAND } \\
\text { LIGHTING CO }\end{array}$ & NY & 11172 & 2517 & 4 & 188 & Operational & 1960 & OIL & SALINE & ONCE THROUGH \\
\hline $\begin{array}{l}\text { PORT } \\
\text { WASHINGTON }\end{array}$ & $\begin{array}{l}\text { WISCONSIN ELEC } \\
\text { POWER CO }\end{array}$ & WI & 20847 & 4040 & 1 & 80 & Operational & 1935 & COAL & FRESH & ONCE THROUGH \\
\hline $\begin{array}{l}\text { PORT } \\
\text { WASHINGTON }\end{array}$ & $\begin{array}{l}\text { WISCONSIN ELEC } \\
\text { POWER CO }\end{array}$ & WI & 20847 & 4040 & 4 & 80 & Operational & 1949 & COAL & FRESH & ONCE THROUGH \\
\hline $\begin{array}{l}\text { PORT } \\
\text { WASHINGTON }\end{array}$ & $\begin{array}{l}\text { WISCONSIN ELEC } \\
\text { POWER CO }\end{array}$ & WI & 20847 & 4040 & 3 & 80 & Operational & 1948 & COAL & FRESH & ONCE THROUGH \\
\hline $\begin{array}{l}\text { PORT } \\
\text { WASHINGTON }\end{array}$ & $\begin{array}{l}\text { WISCONSIN ELEC } \\
\text { POWER CO }\end{array}$ & WI & 20847 & 4040 & 2 & 80 & Operational & 1943 & COAL & FRESH & ONCE THROUGH \\
\hline $\begin{array}{l}\text { PORTLAND } \\
\text { (PA) }\end{array}$ & $\begin{array}{l}\text { METROPOLITAN } \\
\text { EDISON CO }\end{array}$ & PA & 12390 & 3113 & 1 & 172 & Operational & 1958 & COAL & FRESH & ONCE THROUGH \\
\hline $\begin{array}{l}\text { PORTLAND } \\
\text { (PA) }\end{array}$ & $\begin{array}{l}\text { METROPOLITAN } \\
\text { EDISON CO }\end{array}$ & PA & 12390 & 3113 & 2 & 255 & Operational & 1962 & COAL & FRESH & ONCE THROUGH \\
\hline $\begin{array}{l}\text { POSSUM } \\
\text { POINT }\end{array}$ & $\begin{array}{l}\text { VIRGINIA ELEC \& } \\
\text { POWER CO }\end{array}$ & VA & 19876 & 3804 & 4 & 239 & Operational & 1962 & COAL & FRESH & ONCE THROUGH \\
\hline $\begin{array}{l}\text { POSSUM } \\
\text { POINT }\end{array}$ & $\begin{array}{l}\text { VIRGINIA ELEC \& } \\
\text { POWER CO }\end{array}$ & VA & 19876 & 3804 & 3 & 114 & Operational & 1955 & COAL & FRESH & ONCE THROUGH \\
\hline $\begin{array}{l}\text { POSSUM } \\
\text { POINT }\end{array}$ & $\begin{array}{l}\text { VIRGINIA ELEC \& } \\
\text { POWER CO }\end{array}$ & VA & 19876 & 3804 & 2 & 69 & Standby & 1951 & OIL & FRESH & ONCE THROUGH \\
\hline $\begin{array}{l}\text { POSSUM } \\
\text { POINT }\end{array}$ & $\begin{array}{l}\text { VIRGINIA ELEC \& } \\
\text { POWER CO }\end{array}$ & VA & 19876 & 3804 & 1 & 69 & Standby & 1948 & OIL & FRESH & ONCE THROUGH \\
\hline $\begin{array}{l}\text { POTOMAC } \\
\text { RIVER }\end{array}$ & $\begin{array}{l}\text { POTOMAC } \\
\text { ELECTRIC POWER } \\
\end{array}$ & VA & 15270 & 3788 & 2 & 92 & Operational & 1950 & COAL & FRESH & ONCE THROUGH \\
\hline
\end{tabular}




\begin{tabular}{|c|c|c|c|c|c|c|c|c|c|c|c|}
\hline Plant Name & Utility Name & State & Utility/Pl & $n t / U n i$ & $I D$ & $M W$ & Status & Year & Fuel & Water & Cooling System \\
\hline $\begin{array}{l}\text { POTOMAC } \\
\text { RIVER }\end{array}$ & $\begin{array}{l}\text { POTOMAC } \\
\text { ELECTRIC POWER } \\
\sim\end{array}$ & VA & 15270 & 3788 & 3 & 110 & Operational & 1954 & COAL & FRESH & ONCE THROUGH \\
\hline $\begin{array}{l}\text { POTOMAC } \\
\text { RIVER }\end{array}$ & $\begin{array}{l}\text { POTOMAC } \\
\text { ELECTRIC POWER } \\
n\end{array}$ & VA & 15270 & 3788 & 4 & 110 & Operational & 1956 & COAL & FRESH & ONCE THROUGH \\
\hline $\begin{array}{l}\text { POTOMAC } \\
\text { RIVER }\end{array}$ & $\begin{array}{l}\text { POTOMAC } \\
\text { ELECTRIC POWER } \\
\text { n }\end{array}$ & VA & 15270 & 3788 & 5 & 110 & Operational & 1957 & COAL & FRESH & ONCE THROUGH \\
\hline $\begin{array}{l}\text { POTOMAC } \\
\text { RIVER }\end{array}$ & $\begin{array}{l}\text { POTOMAC } \\
\text { ELECTRIC POWER } \\
n\end{array}$ & VA & 15270 & 3788 & 1 & 92 & Operational & 1949 & COAL & FRESH & ONCE THROUGH \\
\hline POTRERO & $\begin{array}{l}\text { PACIFIC GAS \& } \\
\text { ELEC CO }\end{array}$ & $\mathrm{CA}$ & 14328 & 273 & 3 & 218 & Operational & 1965 & GAS & SALINE & ONCE THROUGH \\
\hline POTTER & $\begin{array}{l}\text { BRAINTREE ELEC } \\
\text { LIGHT DEPT }\end{array}$ & MA & 2144 & & 1 & 13 & Standby & 1959 & OIL & BRACKISH & ONCE THROUGH \\
\hline $\begin{array}{l}\text { POWER } \\
\text { HOUSE TWO }\end{array}$ & $\begin{array}{l}\text { NEW ORLEANS } \\
\text { SEW \& WTR BD }\end{array}$ & LA & & & 1 & 6 & Operational & 1928 & GAS & MUNICIPAL & ONCE THROUGH \\
\hline $\begin{array}{l}\text { POWER } \\
\text { HOUSE TWO }\end{array}$ & $\begin{array}{l}\text { NEW ORLEANS } \\
\text { SEW \& WTR BD }\end{array}$ & LA & & & 3 & 15 & Operational & 1940 & GAS & MUNICIPAL & ONCE THROUGH \\
\hline $\begin{array}{l}\text { PRAIRIE } \\
\text { CREEK }\end{array}$ & IES UTILITIES INC & IA & 9162 & 1073 & 1 & 23 & Standby & 1950 & GAS & FRESH & ONCE THROUGH \\
\hline $\begin{array}{l}\text { PRAIRIE } \\
\text { CREEK }\end{array}$ & IES UTILITIES INC & IA & 9162 & 1073 & 2 & 23 & Standby & 1951 & GAS & FRESH & ONCE THROUGH \\
\hline $\begin{array}{l}\text { PRAIRIE } \\
\text { CREEK }\end{array}$ & IES UTILITIES INC & IA & 9162 & 1073 & 3 & 50 & Operational & 1958 & COAL & FRESH & ONCE THROUGH \\
\hline $\begin{array}{l}\text { PRAIRIE } \\
\text { CREEK }\end{array}$ & IES UTILITIES INC & IA & 9162 & 1073 & 4 & 149 & Operational & 1967 & COAL & FRESH & ONCE THROUGH \\
\hline $\begin{array}{l}\text { PRAIRIE } \\
\text { ISLAND }\end{array}$ & $\begin{array}{l}\text { NORTHERN STATES } \\
\text { POWER CO }\end{array}$ & $\mathrm{MN}$ & 13781 & 1925 & 1 & 593 & Operational & 1973 & UR & FRESH & $\begin{array}{l}\text { MIXED MODE (ONCE } \\
\text { THROUGH/COOLING TOWER) }\end{array}$ \\
\hline $\begin{array}{l}\text { PRAIRIE } \\
\text { ISLAND }\end{array}$ & $\begin{array}{l}\text { NORTHERN STATES } \\
\text { POWER CO }\end{array}$ & MN & 13781 & 1925 & 2 & 593 & Operational & 1974 & UR & FRESH & $\begin{array}{l}\text { MIXED MODE (ONCE } \\
\text { THROUGH/COOLING TOWER) }\end{array}$ \\
\hline
\end{tabular}




\begin{tabular}{|c|c|c|c|c|c|c|c|c|c|c|c|}
\hline Plant Name & Utility Name & State & Utility/Pl & $n t / U n i$ & $I D$ & $\boldsymbol{M W}$ & Status & Year & Fuel & Water & Cooling System \\
\hline PRESQUE ISLE & $\begin{array}{l}\text { WISCONSIN ELEC } \\
\text { POWER CO }\end{array}$ & $\mathrm{Ml}$ & 20847 & 1769 & 5 & 90 & Operational & 1974 & COAL & FRESH & ONCE THROUGH \\
\hline PRESQUE ISLE & $\begin{array}{l}\text { WISCONSIN ELEC } \\
\text { POWER CO }\end{array}$ & MI & 20847 & 1769 & 8 & 90 & Operational & 1978 & COAL & FRESH & ONCE THROUGH \\
\hline PRESQUE ISLE & $\begin{array}{l}\text { WISCONSIN ELEC } \\
\text { POWER CO }\end{array}$ & MI & 20847 & 1769 & 9 & 90 & Operational & 1979 & COAL & FRESH & ONCE THROUGH \\
\hline PRESQUE ISLE & $\begin{array}{l}\text { WISCONSIN ELEC } \\
\text { POWER CO }\end{array}$ & MI & 20847 & 1769 & 6 & 90 & Operational & 1975 & COAL & FRESH & ONCE THROUGH \\
\hline PRESQUE ISLE & $\begin{array}{l}\text { WISCONSIN ELEC } \\
\text { POWER CO }\end{array}$ & MI & 20847 & 1769 & 3 & 54.4 & Operational & 1964 & COAL & FRESH & ONCE THROUGH \\
\hline PRESQUE ISLE & $\begin{array}{l}\text { WISCONSIN ELEC } \\
\text { POWER CO }\end{array}$ & MI & 20847 & 1769 & 2 & 37.5 & Operational & 1962 & COAL & FRESH & ONCE THROUGH \\
\hline PRESQUE ISLE & $\begin{array}{l}\text { WISCONSIN ELEC } \\
\text { POWER CO }\end{array}$ & MI & 20847 & 1769 & 1 & 25 & Operational & 1955 & COAL & FRESH & ONCE THROUGH \\
\hline PRESQUE ISLE & $\begin{array}{l}\text { WISCONSIN ELEC } \\
\text { POWER CO }\end{array}$ & MI & 20847 & 1769 & 7 & 90 & Operational & 1978 & COAL & FRESH & ONCE THROUGH \\
\hline PRESQUE ISLE & $\begin{array}{l}\text { WISCONSIN ELEC } \\
\text { POWER CO }\end{array}$ & MI & 20847 & 1769 & 4 & 57.8 & Operational & 1966 & COAL & FRESH & ONCE THROUGH \\
\hline PROVO & $\begin{array}{l}\text { PROVO DEPT OF } \\
\text { UTILITIES }\end{array}$ & UT & 15444 & 3686 & 4 & 7.5 & Operational & 1950 & GAS & UNKNOWN & ONCE THROUGH \\
\hline PUEBLO & $\begin{array}{l}\text { WESTPLAINS } \\
\text { ENERGY }\end{array}$ & $\mathrm{CO}$ & 20391 & 460 & 6 & 15 & Operational & 1949 & GAS & FRESH & $\begin{array}{l}\text { COMBINATION (ONCE } \\
\text { THROUGH/HELPER TOWER) }\end{array}$ \\
\hline PURDOM & $\begin{array}{l}\text { TALLAHASSEE ELEC } \\
\text { DEPT }\end{array}$ & $\mathrm{FL}$ & 18445 & 689 & 5 & 25 & Operational & 1958 & GAS & FRESH & ONCE THROUGH \\
\hline PURDOM & $\begin{array}{l}\text { TALLAHASSEE ELEC } \\
\text { DEPT }\end{array}$ & $\mathrm{FL}$ & 18445 & 689 & 6 & 25 & Operational & 1961 & GAS & FRESH & ONCE THROUGH \\
\hline PURDOM & $\begin{array}{l}\text { TALLAHASSEE ELEC } \\
\text { DEPT }\end{array}$ & $\mathrm{FL}$ & 18445 & 689 & 7 & 50 & Operational & 1966 & GAS & FRESH & ONCE THROUGH \\
\hline
\end{tabular}




\begin{tabular}{|c|c|c|c|c|c|c|c|c|c|c|c|}
\hline Plant Name & Utility Name & State & Utility/Pla & $n t / U n i$ & $I D$ & $M W$ & Status & Year & Fuel & Water & Cooling System \\
\hline QUAD CITIES & $\begin{array}{l}\text { COMMONWEALTH } \\
\text { EDISON CO }\end{array}$ & IL & 4110 & 880 & 1 & 828 & Operational & 1973 & UR & FRESH & ONCE THROUGH \\
\hline QUAD CITIES & $\begin{array}{l}\text { COMMONWEALTH } \\
\text { EDISON CO }\end{array}$ & IL & 4110 & 880 & 2 & 828 & Operational & 1973 & UR & FRESH & ONCE THROUGH \\
\hline $\begin{array}{l}\text { QUINDARO } \\
\text { THREE }\end{array}$ & $\begin{array}{l}\text { KANSAS CITY BD } \\
\text { PUB UTIL }\end{array}$ & KS & 9996 & 1295 & 1 & 81.6 & Operational & 1966 & COAL & FRESH & ONCE THROUGH \\
\hline $\begin{array}{l}\text { QUINDARO } \\
\text { THREE }\end{array}$ & $\begin{array}{l}\text { KANSAS CITY BD } \\
\text { PUB UTIL }\end{array}$ & KS & 9996 & 1295 & 2 & 158 & Operational & 1971 & COAL & FRESH & ONCE THROUGH \\
\hline RALPH GARCIA & KEY WEST UTIL BD & $\mathrm{FL}$ & 10226 & 6583 & 1 & 37 & Operational & 1972 & OIL & GROUND & ONCE THROUGH \\
\hline RATTS & $\begin{array}{l}\text { HOOSIER ENERGY } \\
\text { REC }\end{array}$ & IN & 9267 & 1043 & 1 & 117 & Operational & 1970 & COAL & FRESH & ONCE THROUGH \\
\hline RATTS & $\begin{array}{l}\text { HOOSIER ENERGY } \\
\text { REC }\end{array}$ & IN & 9267 & 1043 & 2 & 117 & Operational & 1970 & COAL & FRESH & ONCE THROUGH \\
\hline RAVENSWOOD & $\begin{array}{l}\text { CONSOLIDATED } \\
\text { EDISON CO }\end{array}$ & NY & 4226 & 2500 & 1 & 400 & Operational & 1963 & GAS & SALINE & ONCE THROUGH \\
\hline RAVENSWOOD & $\begin{array}{l}\text { CONSOLIDATED } \\
\text { EDISON CO }\end{array}$ & NY & 4226 & 2500 & 2 & 400 & Operational & 1963 & GAS & SALINE & ONCE THROUGH \\
\hline RAVENSWOOD & $\begin{array}{l}\text { CONSOLIDATED } \\
\text { EDISON CO }\end{array}$ & NY & 4226 & 2500 & 3 & 1030 & Operational & 1965 & GAS & SALINE & ONCE THROUGH \\
\hline RE BURGER & OHIO EDISON CO & $\mathrm{OH}$ & 13998 & 2864 & 3 & 100 & Operational & 1950 & COAL & FRESH & ONCE THROUGH \\
\hline RE BURGER & OHIO EDISON CO & $\mathrm{OH}$ & 13998 & 2864 & 4 & 160 & Operational & 1955 & COAL & FRESH & ONCE THROUGH \\
\hline RE BURGER & OHIO EDISON CO & $\mathrm{OH}$ & 13998 & 2864 & 5 & 160 & Operational & 1955 & COAL & FRESH & ONCE THROUGH \\
\hline RE RITCHIE & $\begin{array}{l}\text { ARKANSAS POWER } \\
\& \text { LIGHT CO }\end{array}$ & $A R$ & 814 & 173 & 1 & 359 & Standby & 1961 & GAS & FRESH & ONCE THROUGH \\
\hline
\end{tabular}




\begin{tabular}{|c|c|c|c|c|c|c|c|c|c|c|c|}
\hline Plant Name & Utility Name & State & Utility/Pl & $n t / U n i$ & $I D$ & $M W$ & Status & Year & Fuel & Water & Cooling System \\
\hline RE RITCHIE & $\begin{array}{l}\text { ARKANSAS POWER } \\
\& \text { LIGHT CO }\end{array}$ & AR & 814 & 173 & 2 & 545 & Operational & 1967 & GAS & FRESH & ONCE THROUGH \\
\hline RED WING & $\begin{array}{l}\text { NORTHERN STATES } \\
\text { POWER CO }\end{array}$ & MN & 13781 & 1926 & 2 & 11.5 & Operational & 1949 & REFUS & FRESH & ONCE THROUGH \\
\hline RED WING & $\begin{array}{l}\text { NORTHERN STATES } \\
\text { POWER CO }\end{array}$ & MN & 13781 & 1926 & 1 & 11.5 & Operational & 1949 & REFUS & FRESH & ONCE THROUGH \\
\hline $\begin{array}{l}\text { REDONDO } \\
\text { BEACH }\end{array}$ & $\begin{array}{l}\text { SOUTHERN CALIF } \\
\text { EDISON CO }\end{array}$ & CA & 17609 & 356 & 8 & 495 & Operational & 1967 & GAS & SALINE & ONCE THROUGH \\
\hline $\begin{array}{l}\text { REDONDO } \\
\text { BEACH }\end{array}$ & $\begin{array}{l}\text { SOUTHERN CALIF } \\
\text { EDISON CO }\end{array}$ & $\mathrm{CA}$ & 17609 & 356 & 2 & 69 & Standby & 1948 & GAS & SALINE & ONCE THROUGH \\
\hline $\begin{array}{l}\text { REDONDO } \\
\text { BEACH }\end{array}$ & $\begin{array}{l}\text { SOUTHERN CALIF } \\
\text { EDISON CO }\end{array}$ & CA & 17609 & 356 & 3 & 66 & Standby & 1949 & GAS & SALINE & ONCE THROUGH \\
\hline $\begin{array}{l}\text { REDONDO } \\
\text { BEACH }\end{array}$ & $\begin{array}{l}\text { SOUTHERN CALIF } \\
\text { EDISON CO }\end{array}$ & $\mathrm{CA}$ & 17609 & 356 & 4 & 69 & Standby & 1949 & GAS & SALINE & ONCE THROUGH \\
\hline $\begin{array}{l}\text { REDONDO } \\
\text { BEACH }\end{array}$ & $\begin{array}{l}\text { SOUTHERN CALIF } \\
\text { EDISON CO }\end{array}$ & CA & 17609 & 356 & 5 & 156 & Operational & 1954 & GAS & SALINE & ONCE THROUGH \\
\hline $\begin{array}{l}\text { REDONDO } \\
\text { BEACH }\end{array}$ & $\begin{array}{l}\text { SOUTHERN CALIF } \\
\text { EDISON CO }\end{array}$ & CA & 17609 & 356 & 6 & 163 & Operational & 1957 & GAS & SALINE & ONCE THROUGH \\
\hline $\begin{array}{l}\text { REDONDO } \\
\text { BEACH }\end{array}$ & $\begin{array}{l}\text { SOUTHERN CALIF } \\
\text { EDISON CO }\end{array}$ & $\mathrm{CA}$ & 17609 & 356 & 7 & 495 & Operational & 1967 & GAS & SALINE & ONCE THROUGH \\
\hline $\begin{array}{l}\text { REDONDO } \\
\text { BEACH }\end{array}$ & $\begin{array}{l}\text { SOUTHERN CALIF } \\
\text { EDISON CO }\end{array}$ & $\mathrm{CA}$ & 17609 & 356 & 1 & 66 & Standby & 1948 & GAS & SALINE & ONCE THROUGH \\
\hline REID & $\begin{array}{l}\text { BIG RIVERS ELEC } \\
\text { CORP }\end{array}$ & KY & 1692 & 1383 & 1 & 81.6 & Operational & 1965 & COAL & FRESH & ONCE THROUGH \\
\hline RH GORSUCH & $\begin{array}{l}\text { AMERICAN MUNI } \\
\text { POWER OHIO }\end{array}$ & $\mathrm{OH}$ & 40577 & 7286 & 1 & 100 & Operational & 1951 & COAL & FRESH & ONCE THROUGH \\
\hline RH GORSUCH & $\begin{array}{l}\text { AMERICAN MUNI } \\
\text { POWER OHIO }\end{array}$ & $\mathrm{OH}$ & 40577 & 7286 & 2 & 100 & Operational & 1953 & COAL & FRESH & ONCE THROUGH \\
\hline
\end{tabular}




\begin{tabular}{|c|c|c|c|c|c|c|c|c|c|c|c|}
\hline Plant Name & Utility Name & State & Utility/Pl & $n t / U n i$ & $I D$ & $M W$ & Status & Year & Fuel & Water & Cooling Systen \\
\hline RIVER ROUGE & $\begin{array}{l}\text { DETROIT EDISON } \\
\text { CO }\end{array}$ & MI & 5109 & 1740 & 1 & 283 & Standby & 1956 & GAS & FRESH & ONCE THROUGH \\
\hline RIVER ROUGE & $\begin{array}{l}\text { DETROIT EDISON } \\
\text { CO }\end{array}$ & MI & 5109 & 1740 & 2 & 293 & Operational & 1957 & COAL & FRESH & ONCE THROUGH \\
\hline RIVER ROUGE & $\begin{array}{l}\text { DETROIT EDISON } \\
\text { CO }\end{array}$ & MI & 5109 & 1740 & 3 & 358 & Operational & 1958 & COAL & FRESH & ONCE THROUGH \\
\hline RIVER STREET & $\begin{array}{l}\text { MENASHA ELEC \& } \\
\text { WATER UTIL }\end{array}$ & WI & 12298 & 4127 & 3 & 8 & Standby & 1956 & COAL & FRESH & ONCE THROUGH \\
\hline RIVER STREET & $\begin{array}{l}\text { MENASHA ELEC \& } \\
\text { WATER UTIL }\end{array}$ & WI & 12298 & 4127 & 4 & 14 & Operational & 1964 & COAL & FRESH & ONCE THROUGH \\
\hline $\begin{array}{l}\text { RIVERBEND } \\
\text { (NC) }\end{array}$ & DUKE POWER CO & $\mathrm{NC}$ & 5416 & 2732 & 4 & 100 & Standby & 1952 & COAL & FRESH & ONCE THROUGH \\
\hline $\begin{array}{l}\text { RIVERBEND } \\
\text { (NC) }\end{array}$ & DUKE POWER CO & $\mathrm{NC}$ & 5416 & 2732 & 7 & 133 & Standby & 1954 & COAL & FRESH & ONCE THROUGH \\
\hline $\begin{array}{l}\text { RIVERBEND } \\
\text { (NC) }\end{array}$ & DUKE POWER CO & $\mathrm{NC}$ & 5416 & 2732 & 6 & 133 & Standby & 1954 & COAL & FRESH & ONCE THROUGH \\
\hline $\begin{array}{l}\text { RIVERBEND } \\
\text { (NC) }\end{array}$ & DUKE POWER CO & $\mathrm{NC}$ & 5416 & 2732 & 5 & 100 & Operational & 1952 & COAL & FRESH & ONCE THROUGH \\
\hline $\begin{array}{l}\text { RIVERSIDE } \\
\text { (GA) }\end{array}$ & $\begin{array}{l}\text { SAVANNAH ELEC \& } \\
\text { POWER CO }\end{array}$ & GA & 16687 & 734 & 4 & 15 & Operational & 1926 & GAS & BRACKISH & ONCE THROUGH \\
\hline $\begin{array}{l}\text { RIVERSIDE } \\
\text { (GA) }\end{array}$ & $\begin{array}{l}\text { SAVANNAH ELEC \& } \\
\text { POWER CO }\end{array}$ & GA & 16687 & 734 & 5 & 7.5 & Operational & 1936 & GAS & BRACKISH & ONCE THROUGH \\
\hline $\begin{array}{l}\text { RIVERSIDE } \\
\text { (GA) }\end{array}$ & $\begin{array}{l}\text { SAVANNAH ELEC \& } \\
\text { POWER CO }\end{array}$ & GA & 16687 & 734 & 6 & 24.8 & Standby & 1949 & GAS & BRACKISH & ONCE THROUGH \\
\hline $\begin{array}{l}\text { RIVERSIDE } \\
\text { (GA) }\end{array}$ & $\begin{array}{l}\text { SAVANNAH ELEC \& } \\
\text { POWER CO }\end{array}$ & GA & 16687 & 734 & 7 & 21.3 & Standby & 1954 & GAS & BRACKISH & ONCE THROUGH \\
\hline $\begin{array}{l}\text { RIVERSIDE } \\
\text { (GA) }\end{array}$ & $\begin{array}{l}\text { SAVANNAH ELEC \& } \\
\text { POWER CO }\end{array}$ & GA & 16687 & 734 & 8 & 37.5 & Standby & 1956 & GAS & BRACKISH & ONCE THROUGH \\
\hline
\end{tabular}




\begin{tabular}{|c|c|c|c|c|c|c|c|c|c|c|c|}
\hline Plant Name & Utility Name & State & Utility/P & $n t / U n i$ & $I D$ & $\boldsymbol{M W}$ & Status & Year & Fuel & Water & Cooling Systen \\
\hline RIVERSIDE (IA) & $\begin{array}{l}\text { IOWA-ILLINOIS GAS } \\
\& \text { ELEC }\end{array}$ & IA & 9438 & 1081 & 5 & 136 & Operational & 1961 & COAL & FRESH & ONCE THROUGH \\
\hline $\begin{array}{l}\text { RIVERSIDE } \\
\text { (MD) }\end{array}$ & $\begin{array}{l}\text { BALTIMORE GAS \& } \\
\text { ELEC CO }\end{array}$ & MD & 1167 & 1559 & 4 & 72.3 & Operational & 1951 & GAS & BRACKISH & ONCE THROUGH \\
\hline $\begin{array}{l}\text { RIVERSIDE } \\
\text { (MN) }\end{array}$ & $\begin{array}{l}\text { NORTHERN STATES } \\
\text { POWER CO }\end{array}$ & $\mathrm{MN}$ & 13781 & 1927 & 8 & 239 & Operational & 1964 & COAL & FRESH & ONCE THROUGH \\
\hline $\begin{array}{l}\text { RIVERSIDE } \\
\text { (MN) }\end{array}$ & $\begin{array}{l}\text { NORTHERN STATES } \\
\text { POWER CO }\end{array}$ & $\mathrm{MN}$ & 13781 & 1927 & 7 & 137 & Operational & 1987 & COAL & FRESH & ONCE THROUGH \\
\hline RIVERTON & $\begin{array}{l}\text { EMPIRE DISTRICT } \\
\text { ELEC CO }\end{array}$ & KS & 5860 & 1239 & 7 & 37.5 & Operational & 1950 & COAL & FRESH & ONCE THROUGH \\
\hline RIVERTON & $\begin{array}{l}\text { EMPIRE DISTRICT } \\
\text { ELEC CO }\end{array}$ & KS & 5860 & 1239 & 8 & 50 & Operational & 1954 & COAL & FRESH & ONCE THROUGH \\
\hline RIVESVILLE & $\begin{array}{l}\text { MONONGAHELA } \\
\text { POWER CO }\end{array}$ & WV & 12796 & 3945 & 6 & 74.8 & Operational & 1951 & COAL & FRESH & ONCE THROUGH \\
\hline RIVESVILLE & $\begin{array}{l}\text { MONONGAHELA } \\
\text { POWER CO }\end{array}$ & WV & 12796 & 3945 & 5 & 35 & Operational & 1944 & COAL & FRESH & ONCE THROUGH \\
\hline RIVIERA & $\begin{array}{l}\text { FLORIDA POWER \& } \\
\text { LIGHT CO }\end{array}$ & $\mathrm{FL}$ & 6452 & 619 & 4 & 310 & Operational & 1963 & OIL & SALINE & ONCE THROUGH \\
\hline RIVIERA & $\begin{array}{l}\text { FLORIDA POWER \& } \\
\text { LIGHT CO }\end{array}$ & $\mathrm{FL}$ & 6452 & 619 & 3 & 310 & Operational & 1962 & OIL & SALINE & ONCE THROUGH \\
\hline ROCK RIVER & $\begin{array}{l}\text { WISCONSIN } \\
\text { POWER \& LIGHT }\end{array}$ & WI & 20856 & 4057 & 1 & 75 & Operational & 1954 & COAL & FRESH & ONCE THROUGH \\
\hline ROCK RIVER & $\begin{array}{l}\text { WISCONSIN } \\
\text { POWER \& LIGHT }\end{array}$ & WI & 20856 & 4057 & 2 & 75 & Operational & 1955 & COAL & FRESH & ONCE THROUGH \\
\hline ROSETON & $\begin{array}{l}\text { CENTRAL HUDSON } \\
\text { GAS \& ELEC }\end{array}$ & NY & 3249 & 8006 & 2 & 621 & Operational & 1974 & OIL & BRACKISH & ONCE THROUGH \\
\hline ROSETON & $\begin{array}{l}\text { CENTRAL HUDSON } \\
\text { GAS \& ELEC }\end{array}$ & NY & 3249 & 8006 & 1 & 621 & Operational & 1974 & OIL & BRACKISH & ONCE THROUGH \\
\hline
\end{tabular}




\begin{tabular}{|c|c|c|c|c|c|c|c|c|c|c|c|}
\hline Plant Name & Utility Name & State & Utility/Pl & $n t / U n i$ & $I D$ & $\boldsymbol{M W}$ & Status & Year & Fuel & Water & Cooling System \\
\hline ROXBORO & $\begin{array}{l}\text { CAROLINA POWER } \\
\text { \& LIGHT CO }\end{array}$ & NC & 3046 & 2712 & 3 & 745 & Operational & 1973 & COAL & FRESH & $\begin{array}{l}\text { COMBINATION (ONCE } \\
\text { THROUGH/HELPER TOWER) }\end{array}$ \\
\hline ROXBORO & $\begin{array}{l}\text { CAROLINA POWER } \\
\text { \& LIGHT CO }\end{array}$ & NC & 3046 & 2712 & 2 & 657 & Operational & 1968 & COAL & FRESH & ONCE THROUGH \\
\hline ROXBORO & $\begin{array}{l}\text { CAROLINA POWER } \\
\& \text { LIGHT CO }\end{array}$ & $\mathrm{NC}$ & 3046 & 2712 & 1 & 411 & Operational & 1966 & COAL & FRESH & ONCE THROUGH \\
\hline RP SMITH & $\begin{array}{l}\text { POTOMAC EDISON } \\
\text { CO }\end{array}$ & MD & 15263 & 1570 & 3 & 34.5 & Operational & 1947 & COAL & FRESH & ONCE THROUGH \\
\hline RP SMITH & $\begin{array}{l}\text { POTOMAC EDISON } \\
\text { CO }\end{array}$ & MD & 15263 & 1570 & 4 & 75 & Operational & 1957 & COAL & FRESH & ONCE THROUGH \\
\hline RUSH ISLAND & UNION ELECTRIC CO & MO & 19436 & 6155 & 1 & 621 & Operational & 1976 & COAL & FRESH & ONCE THROUGH \\
\hline RUSH ISLAND & UNION ELECTRIC CO & MO & 19436 & 6155 & 2 & 621 & Operational & 1977 & COAL & FRESH & ONCE THROUGH \\
\hline RUSSELL (NY) & $\begin{array}{l}\text { ROCHESTER GAS \& } \\
\text { ELEC CORP }\end{array}$ & NY & 16183 & 2642 & 1 & 46 & Operational & 1949 & COAL & FRESH & ONCE THROUGH \\
\hline RUSSELL (NY) & $\begin{array}{l}\text { ROCHESTER GAS \& } \\
\text { ELEC CORP }\end{array}$ & NY & 16183 & 2642 & 2 & 62.5 & Operational & 1951 & COAL & FRESH & ONCE THROUGH \\
\hline RUSSELL (NY) & $\begin{array}{l}\text { ROCHESTER GAS \& } \\
\text { ELEC CORP }\end{array}$ & NY & 16183 & 2642 & 3 & 62.5 & Operational & 1953 & COAL & FRESH & ONCE THROUGH \\
\hline RUSSELL (NY) & $\begin{array}{l}\text { ROCHESTER GAS \& } \\
\text { ELEC CORP }\end{array}$ & NY & 16183 & 2642 & 4 & 81.6 & Operational & 1957 & COAL & FRESH & ONCE THROUGH \\
\hline RW MILLER & $\begin{array}{l}\text { BRAZOS ELEC } \\
\text { POWER COOP }\end{array}$ & $\mathrm{TX}$ & 2172 & 3628 & 2 & 100 & Operational & 1972 & GAS & FRESH & ONCE THROUGH \\
\hline RW MILLER & $\begin{array}{l}\text { BRAZOS ELEC } \\
\text { POWER COOP }\end{array}$ & $\mathrm{TX}$ & 2172 & 3628 & 3 & 200 & Operational & 1975 & GAS & FRESH & ONCE THROUGH \\
\hline RW MILLER & $\begin{array}{l}\text { BRAZOS ELEC } \\
\text { POWER COOP }\end{array}$ & $\mathrm{TX}$ & 2172 & 3628 & 1 & 66 & Operational & 1968 & GAS & FRESH & ONCE THROUGH \\
\hline
\end{tabular}




\begin{tabular}{|c|c|c|c|c|c|c|c|c|c|c|c|}
\hline Plant Name & Utility Name & State & Utility/Pl & nt/Unit & $I D$ & $M W$ & Status & Year & Fuel & Water & Cooling System \\
\hline SABINE & $\begin{array}{l}\text { GULF STATES } \\
\text { UTILITIES }\end{array}$ & TX & 7806 & 3459 & 1 & 239 & Operational & 1962 & GAS & BRACKISH & ONCE THROUGH \\
\hline SABINE & $\begin{array}{l}\text { GULF STATES } \\
\text { UTILITIES }\end{array}$ & TX & 7806 & 3459 & 2 & 239 & Operational & 1962 & GAS & BRACKISH & ONCE THROUGH \\
\hline SABINE & $\begin{array}{l}\text { GULF STATES } \\
\text { UTILITIES }\end{array}$ & TX & 7806 & 3459 & 3 & 473 & Operational & 1966 & GAS & BRACKISH & ONCE THROUGH \\
\hline SABINE & $\begin{array}{l}\text { GULF STATES } \\
\text { UTILITIES }\end{array}$ & TX & 7806 & 3459 & 4 & 592 & Operational & 1974 & GAS & BRACKISH & ONCE THROUGH \\
\hline SAINT CROIX & $\begin{array}{l}\text { VIRGIN ISLANDS } \\
\text { WTR \& PWR }\end{array}$ & VI & 19879 & & 1 & 15 & Operational & 1966 & OIL & SALINE & ONCE THROUGH \\
\hline SAINT CROIX & $\begin{array}{l}\text { VIRGIN ISLANDS } \\
\text { WTR \& PWR }\end{array}$ & VI & 19879 & & 2 & 15 & Operational & 1966 & OIL & SALINE & ONCE THROUGH \\
\hline $\begin{array}{l}\text { SAINT } \\
\text { THOMAS A }\end{array}$ & $\begin{array}{l}\text { VIRGIN ISLANDS } \\
\text { WTR \& PWR }\end{array}$ & VI & 19879 & & 1 & 62 & Operational & 1975 & OIL & SALINE & ONCE THROUGH \\
\hline SALEM (NJ) & $\begin{array}{l}\text { PUBLIC SERVICE } \\
\text { ELEC \& GAS }\end{array}$ & $\mathrm{NJ}$ & 15477 & 2410 & 1 & 1170 & Operational & 1977 & UR & BRACKISH & ONCE THROUGH \\
\hline SALEM (NJ) & $\begin{array}{l}\text { PUBLIC SERVICE } \\
\text { ELEC \& GAS }\end{array}$ & $\mathrm{NJ}$ & 15477 & 2410 & 2 & 1170 & Operational & 1981 & UR & BRACKISH & ONCE THROUGH \\
\hline $\begin{array}{l}\text { SALEM } \\
\text { HARBOR }\end{array}$ & $\begin{array}{l}\text { NEW ENGLAND } \\
\text { POWER CO }\end{array}$ & MA & 13433 & 1626 & 1 & 81.9 & Operational & 1952 & COAL & SALINE & ONCE THROUGH \\
\hline $\begin{array}{l}\text { SALEM } \\
\text { HARBOR }\end{array}$ & $\begin{array}{l}\text { NEW ENGLAND } \\
\text { POWER CO }\end{array}$ & MA & 13433 & 1626 & 4 & 476 & Operational & 1972 & OIL & SALINE & ONCE THROUGH \\
\hline $\begin{array}{l}\text { SALEM } \\
\text { HARBOR }\end{array}$ & $\begin{array}{l}\text { NEW ENGLAND } \\
\text { POWER CO }\end{array}$ & MA & 13433 & 1626 & 3 & 166 & Operational & 1958 & COAL & SALINE & ONCE THROUGH \\
\hline $\begin{array}{l}\text { SALEM } \\
\text { HARBOR }\end{array}$ & $\begin{array}{l}\text { NEW ENGLAND } \\
\text { POWER CO }\end{array}$ & MA & 13433 & 1626 & 2 & 82 & Operational & 1952 & COAL & SALINE & ONCE THROUGH \\
\hline SAM BERTRON & $\begin{array}{l}\text { HOUSTON } \\
\text { LIGHTING \& POWER }\end{array}$ & TX & 8901 & 3468 & 1 & 188 & Operational & 1958 & GAS & BRACKISH & $\begin{array}{l}\text { COMBINATION (ONCE } \\
\text { THROUGH/HELPER TOWER) }\end{array}$ \\
\hline
\end{tabular}




\begin{tabular}{|c|c|c|c|c|c|c|c|c|c|c|c|}
\hline Plant Name & Utility Name & State & Utility/Pl & it/Uni & $I D$ & $\boldsymbol{M W}$ & Status & Year & Fuel & Water & Cooling System \\
\hline SAM BERTRON & $\begin{array}{l}\text { HOUSTON } \\
\text { LIGHTING \& POWER }\end{array}$ & TX & 8901 & 3468 & 2 & 188 & Operational & 1956 & GAS & BRACKISH & $\begin{array}{l}\text { COMBINATION (ONCE } \\
\text { THROUGH/HELPER TOWER) }\end{array}$ \\
\hline SAM BERTRON & $\begin{array}{l}\text { HOUSTON } \\
\text { LIGHTING \& POWER }\end{array}$ & TX & 8901 & 3468 & 3 & 225 & Operational & 1959 & GAS & BRACKISH & $\begin{array}{l}\text { COMBINATION (ONCE } \\
\text { THROUGH/HELPER TOWER) }\end{array}$ \\
\hline SAM BERTRON & $\begin{array}{l}\text { HOUSTON } \\
\text { LIGHTING \& POWER }\end{array}$ & TX & 8901 & 3468 & 4 & 225 & Operational & 1960 & GAS & BRACKISH & $\begin{array}{l}\text { COMBINATION (ONCE } \\
\text { THROUGH/HELPER TOWER) }\end{array}$ \\
\hline SAM RAYBURN & $\begin{array}{l}\text { SOUTH TEXAS ELEC } \\
\text { COOP }\end{array}$ & TX & 17583 & 3631 & 3 & 22 & Operational & 1965 & GAS & FRESH & $\begin{array}{l}\text { COMBINATION (ONCE } \\
\text { THROUGH/SPRAY POND) }\end{array}$ \\
\hline $\begin{array}{l}\text { SAN ANGELO } \\
\text { SC }\end{array}$ & $\begin{array}{l}\text { WEST TEXAS UTIL } \\
\text { CO }\end{array}$ & TX & 20404 & 3527 & 2 & 101 & Operational & 1966 & GAS & FRESH & ONCE THROUGH \\
\hline SAN JUAN (PR) & $\begin{array}{l}\text { PUERTO RICO ELEC } \\
\text { PWR AUTH }\end{array}$ & PR & 15497 & & 5 & 44 & Operational & 1956 & OIL & SALINE & ONCE THROUGH \\
\hline SAN JUAN (PR) & $\begin{array}{l}\text { PUERTO RICO ELEC } \\
\text { PWR AUTH }\end{array}$ & PR & 15497 & & 9 & 114 & Operational & 1968 & OIL & SALINE & ONCE THROUGH \\
\hline SAN JUAN (PR) & $\begin{array}{l}\text { PUERTO RICO ELEC } \\
\text { PWR AUTH }\end{array}$ & PR & 15497 & & 8 & 114 & Operational & 1966 & OIL & SALINE & ONCE THROUGH \\
\hline SAN JUAN (PR) & $\begin{array}{l}\text { PUERTO RICO ELEC } \\
\text { PWR AUTH }\end{array}$ & PR & 15497 & & 7 & 114 & Operational & 1966 & OIL & SALINE & ONCE THROUGH \\
\hline SAN JUAN (PR) & $\begin{array}{l}\text { PUERTO RICO ELEC } \\
\text { PWR AUTH }\end{array}$ & PR & 15497 & & 10 & 114 & Operational & 1969 & OIL & SALINE & ONCE THROUGH \\
\hline SAN JUAN (PR) & $\begin{array}{l}\text { PUERTO RICO ELEC } \\
\text { PWR AUTH }\end{array}$ & PR & 15497 & & 6 & 44 & Operational & 1957 & OIL & SALINE & ONCE THROUGH \\
\hline SAN ONOFRE & $\begin{array}{l}\text { SOUTHERN CALIF } \\
\text { EDISON CO }\end{array}$ & $\mathrm{CA}$ & 17609 & 360 & 2 & 1127 & Operational & 1983 & UR & SALINE & ONCE THROUGH \\
\hline SAN ONOFRE & $\begin{array}{l}\text { SOUTHERN CALIF } \\
\text { EDISON CO }\end{array}$ & $\mathrm{CA}$ & 17609 & 360 & 3 & 1127 & Operational & 1984 & UR & SALINE & ONCE THROUGH \\
\hline SANFORD (FL) & $\begin{array}{l}\text { FLORIDA POWER \& } \\
\text { LIGHT CO }\end{array}$ & FL & 6452 & 620 & 3 & 156 & Operational & 1959 & OIL & FRESH & ONCE THROUGH \\
\hline
\end{tabular}




\begin{tabular}{|c|c|c|c|c|c|c|c|c|c|c|c|}
\hline Plant Name & Utility Name & State & Utility/P & $n t / U n i$ & $I D$ & $M W$ & Status & Year & Fuel & Water & Cooling System \\
\hline SAYREVILLE & $\begin{array}{l}\text { JERSEY CENT } \\
\text { POWER \& LIGHT }\end{array}$ & NJ & 9726 & 2390 & 4 & 123 & Operational & 1955 & GAS & BRACKISH & ONCE THROUGH \\
\hline SAYREVILLE & $\begin{array}{l}\text { JERSEY CENT } \\
\text { POWER \& LIGHT }\end{array}$ & NJ & 9726 & 2390 & 5 & 125 & Operational & 1958 & GAS & BRACKISH & ONCE THROUGH \\
\hline $\begin{array}{l}\text { SCATTERGOO } \\
\text { D }\end{array}$ & $\begin{array}{l}\text { LOS ANGELES DEPT } \\
\text { WTR PWR }\end{array}$ & $\mathrm{CA}$ & 11208 & 404 & 1 & 163 & Operational & 1958 & GAS & SALINE & ONCE THROUGH \\
\hline $\begin{array}{l}\text { SCATTERGOO } \\
\text { D }\end{array}$ & $\begin{array}{l}\text { LOS ANGELES DEPT } \\
\text { WTR PWR }\end{array}$ & $\mathrm{CA}$ & 11208 & 404 & 2 & 163 & Operational & 1959 & GAS & SALINE & ONCE THROUGH \\
\hline $\begin{array}{l}\text { SCATTERGOO } \\
\text { D }\end{array}$ & $\begin{array}{l}\text { LOS ANGELES DEPT } \\
\text { WTR PWR }\end{array}$ & $\mathrm{CA}$ & 11208 & 404 & 3 & 497 & Operational & 1974 & GAS & SALINE & ONCE THROUGH \\
\hline SCHILLER & $\begin{array}{l}\text { PUBLIC SERV NEW } \\
\text { HAMPSHIRE }\end{array}$ & $\mathrm{NH}$ & 15472 & 2367 & 4 & 50 & Operational & 1952 & COAL & BRACKISH & ONCE THROUGH \\
\hline SCHILLER & $\begin{array}{l}\text { PUBLIC SERV NEW } \\
\text { HAMPSHIRE }\end{array}$ & $\mathrm{NH}$ & 15472 & 2367 & 5 & 50 & Operational & 1955 & COAL & BRACKISH & ONCE THROUGH \\
\hline SCHILLER & $\begin{array}{l}\text { PUBLIC SERV NEW } \\
\text { HAMPSHIRE }\end{array}$ & $\mathrm{NH}$ & 15472 & 2367 & 6 & 50 & Operational & 1957 & COAL & BRACKISH & ONCE THROUGH \\
\hline SCHOLZ & GULF POWER CO & $\mathrm{FL}$ & 7801 & 642 & 1 & 49 & Operational & 1953 & COAL & FRESH & ONCE THROUGH \\
\hline SCHOLZ & GULF POWER CO & $\mathrm{FL}$ & 7801 & 642 & 2 & 49 & Operational & 1953 & COAL & FRESH & ONCE THROUGH \\
\hline SCHUYLER & $\begin{array}{l}\text { SCHUYLER DEPT } \\
\text { UTILITIES }\end{array}$ & NE & 16778 & & 1 & 5 & Standby & 1958 & OIL & MUNICIPAL & ONCE THROUGH \\
\hline SCHUYLER & $\begin{array}{l}\text { SCHUYLER DEPT } \\
\text { UTILITIES }\end{array}$ & NE & 16778 & & 2 & 2.5 & Standby & 1955 & OIL & MUNICIPAL & ONCE THROUGH \\
\hline SCHUYLKILL & PECO ENERGY CO & PA & 14940 & 3169 & 1 & 190 & Operational & 1958 & OIL & FRESH & ONCE THROUGH \\
\hline SEABROOK & $\begin{array}{l}\text { NORTH ATLANTIC } \\
\text { ENERGY }\end{array}$ & $\mathrm{NH}$ & 13714 & 6115 & 1 & 1197 & Operational & 1990 & UR & SALINE & ONCE THROUGH \\
\hline
\end{tabular}




\begin{tabular}{|c|c|c|c|c|c|c|c|c|c|c|c|}
\hline Plant Name & Utility Name & State & Utility/Pl & $n t / U n i$ & $I D$ & $M W$ & Status & Year & Fuel & Water & Cooling System \\
\hline SEBRING & $\begin{array}{l}\text { TAMPA ELECTRIC } \\
\text { CO }\end{array}$ & $\mathrm{FL}$ & 18454 & 747 & 1 & 12.7 & Operational & 1966 & GAS & FRESH & ONCE THROUGH \\
\hline SEQUOYAH & $\begin{array}{l}\text { TENNESSEE } \\
\text { VALLEY AUTH }\end{array}$ & $\mathrm{TN}$ & 18642 & 6152 & 2 & 1221 & Operational & 1982 & UR & FRESH & $\begin{array}{l}\text { COMBINATION (ONCE } \\
\text { THROUGH/HELPER TOWER) }\end{array}$ \\
\hline SEQUOYAH & $\begin{array}{l}\text { TENNESSEE } \\
\text { VALLEY AUTH }\end{array}$ & $\mathrm{TN}$ & 18642 & 6152 & 1 & 1221 & Operational & 1981 & UR & FRESH & $\begin{array}{l}\text { COMBINATION (ONCE } \\
\text { THROUGH/HELPER TOWER) }\end{array}$ \\
\hline SEWARD & $\begin{array}{l}\text { PENNSYLVANIA } \\
\text { ELEC CO }\end{array}$ & PA & 14711 & 3130 & 4 & 62 & Operational & 1950 & COAL & FRESH & ONCE THROUGH \\
\hline SEWARD & $\begin{array}{l}\text { PENNSYLVANIA } \\
\text { ELEC CO }\end{array}$ & PA & 14711 & 3130 & 5 & 156 & Operational & 1957 & COAL & FRESH & ONCE THROUGH \\
\hline SEWAREN & $\begin{array}{l}\text { PUBLIC SERVICE } \\
\text { ELEC \& GAS }\end{array}$ & NJ & 15477 & 2411 & 1 & 111 & Operational & 1948 & GAS & SALINE & ONCE THROUGH \\
\hline SEWAREN & $\begin{array}{l}\text { PUBLIC SERVICE } \\
\text { ELEC \& GAS }\end{array}$ & NJ & 15477 & 2411 & 2 & 108 & Operational & 1948 & GAS & SALINE & ONCE THROUGH \\
\hline SEWAREN & $\begin{array}{l}\text { PUBLIC SERVICE } \\
\text { ELEC \& GAS }\end{array}$ & NJ & 15477 & 2411 & 3 & 116 & Operational & 1949 & GAS & SALINE & ONCE THROUGH \\
\hline SEWAREN & $\begin{array}{l}\text { PUBLIC SERVICE } \\
\text { ELEC \& GAS }\end{array}$ & NJ & 15477 & 2411 & 4 & 127 & Operational & 1951 & GAS & SALINE & ONCE THROUGH \\
\hline SHAWNEE (KY) & $\begin{array}{l}\text { TENNESSEE } \\
\text { VALLEY AUTH }\end{array}$ & KY & 18642 & 1379 & 9 & 175 & Operational & 1955 & COAL & FRESH & ONCE THROUGH \\
\hline SHAWNEE (KY) & $\begin{array}{l}\text { TENNESSEE } \\
\text { VALLEY AUTH }\end{array}$ & KY & 18642 & 1379 & 10 & 175 & Operational & 1956 & COAL & FRESH & ONCE THROUGH \\
\hline SHAWNEE (KY) & $\begin{array}{l}\text { TENNESSEE } \\
\text { VALLEY AUTH }\end{array}$ & KY & 18642 & 1379 & 8 & 175 & Operational & 1955 & COAL & FRESH & ONCE THROUGH \\
\hline SHAWNEE (KY) & $\begin{array}{l}\text { TENNESSEE } \\
\text { VALLEY AUTH }\end{array}$ & KY & 18642 & 1379 & 7 & 175 & Operational & 1954 & COAL & FRESH & ONCE THROUGH \\
\hline SHAWNEE (KY) & $\begin{array}{l}\text { TENNESSEE } \\
\text { VALLEY AUTH }\end{array}$ & KY & 18642 & 1379 & 6 & 175 & Operational & 1954 & COAL & FRESH & ONCE THROUGH \\
\hline
\end{tabular}




\begin{tabular}{|c|c|c|c|c|c|c|c|c|c|c|c|}
\hline Plant Name & Utility Name & State & Utility/Pl & $n t / U n i$ & $I D$ & $M W$ & Status & Year & Fuel & Water & Cooling System \\
\hline SHAWNEE (KY) & $\begin{array}{l}\text { TENNESSEE } \\
\text { VALLEY AUTH }\end{array}$ & KY & 18642 & 1379 & 4 & 175 & Operational & 1954 & COAL & FRESH & ONCE THROUGH \\
\hline SHAWNEE (KY) & $\begin{array}{l}\text { TENNESSEE } \\
\text { VALLEY AUTH }\end{array}$ & KY & 18642 & 1379 & 3 & 175 & Operational & 1953 & COAL & FRESH & ONCE THROUGH \\
\hline SHAWNEE (KY) & $\begin{array}{l}\text { TENNESSEE } \\
\text { VALLEY AUTH }\end{array}$ & KY & 18642 & 1379 & 2 & 175 & Operational & 1953 & COAL & FRESH & ONCE THROUGH \\
\hline SHAWNEE (KY) & $\begin{array}{l}\text { TENNESSEE } \\
\text { VALLEY AUTH }\end{array}$ & KY & 18642 & 1379 & 1 & 175 & Operational & 1953 & COAL & FRESH & ONCE THROUGH \\
\hline SHAWNEE (KY) & $\begin{array}{l}\text { TENNESSEE } \\
\text { VALLEY AUTH }\end{array}$ & KY & 18642 & 1379 & 5 & 175 & Operational & 1954 & COAL & FRESH & ONCE THROUGH \\
\hline SHAWVILLE & $\begin{array}{l}\text { PENNSYLVANIA } \\
\text { ELEC CO }\end{array}$ & PA & 14711 & 3131 & 1 & 125 & Operational & 1954 & COAL & FRESH & $\begin{array}{l}\text { COMBINATION (ONCE } \\
\text { THROUGH/HELPER TOWER) }\end{array}$ \\
\hline SHAWVILLE & $\begin{array}{l}\text { PENNSYLVANIA } \\
\text { ELEC CO }\end{array}$ & PA & 14711 & 3131 & 2 & 125 & Operational & 1954 & COAL & FRESH & $\begin{array}{l}\text { COMBINATION (ONCE } \\
\text { THROUGH/HELPER TOWER) }\end{array}$ \\
\hline SHAWVILLE & $\begin{array}{l}\text { PENNSYLVANIA } \\
\text { ELEC CO }\end{array}$ & PA & 14711 & 3131 & 3 & 188 & Operational & 1959 & COAL & FRESH & $\begin{array}{l}\text { COMBINATION (ONCE } \\
\text { THROUGH/HELPER TOWER) }\end{array}$ \\
\hline SHAWVILLE & $\begin{array}{l}\text { PENNSYLVANIA } \\
\text { ELEC CO }\end{array}$ & PA & 14711 & 3131 & 4 & 188 & Operational & 1960 & COAL & FRESH & $\begin{array}{l}\text { COMBINATION (ONCE } \\
\text { THROUGH/HELPER TOWER) }\end{array}$ \\
\hline SHIPMAN & $\begin{array}{l}\text { HAWAII ELEC LIGHT } \\
\text { CO }\end{array}$ & $\mathrm{HI}$ & 8287 & 6478 & 1 & 3.5 & Operational & 1943 & OIL & GROUND & ONCE THROUGH \\
\hline SHIPMAN & $\begin{array}{l}\text { HAWAII ELEC LIGHT } \\
\text { CO }\end{array}$ & $\mathrm{HI}$ & 8287 & 6478 & 3 & 7.5 & Operational & 1955 & OIL & GROUND & ONCE THROUGH \\
\hline SHIPMAN & $\begin{array}{l}\text { HAWAII ELEC LIGHT } \\
\text { CO }\end{array}$ & $\mathrm{HI}$ & 8287 & 6478 & 4 & 7.5 & Operational & 1958 & OIL & GROUND & ONCE THROUGH \\
\hline SHIRAS & $\begin{array}{l}\text { MARQUETTE BD LT } \\
\text { \& POWER }\end{array}$ & MI & 11701 & 1843 & 1 & 12.5 & Operational & 1967 & COAL & FRESH & ONCE THROUGH \\
\hline SHIRAS & $\begin{array}{l}\text { MARQUETTE BD LT } \\
\& \text { POWER }\end{array}$ & MI & 11701 & 1843 & 2 & 21 & Operational & 1972 & COAL & FRESH & ONCE THROUGH \\
\hline
\end{tabular}




\begin{tabular}{|c|c|c|c|c|c|c|c|c|c|c|c|}
\hline Plant Name & Utility Name & State & Utility/P & $n t / U n i$ & $I D$ & $\boldsymbol{M W}$ & Status & Year & Fuel & Water & Cooling System \\
\hline SHIRAS & $\begin{array}{l}\text { MARQUETTE BD LT } \\
\text { \& POWER }\end{array}$ & MI & 11701 & 1843 & 3 & 43.7 & Operational & 1983 & COAL & FRESH & ONCE THROUGH \\
\hline SHUFFLETON & $\begin{array}{l}\text { PUGET SOUND } \\
\text { POWER \& LIGHT }\end{array}$ & WA & & & 2 & 43.8 & Standby & 1930 & OIL & FRESH & ONCE THROUGH \\
\hline SIBLEY (MO) & $\begin{array}{l}\text { UTILICORP UNITED } \\
\text { INC }\end{array}$ & MO & 12698 & 2094 & 1 & 55 & Operational & 1960 & COAL & FRESH & ONCE THROUGH \\
\hline SIBLEY (MO) & $\begin{array}{l}\text { UTILICORP UNITED } \\
\text { INC }\end{array}$ & MO & 12698 & 2094 & 2 & 50 & Operational & 1962 & COAL & FRESH & ONCE THROUGH \\
\hline SIBLEY (MO) & $\begin{array}{l}\text { UTILICORP UNITED } \\
\text { INC }\end{array}$ & MO & 12698 & 2094 & 3 & 419 & Operational & 1969 & COAL & FRESH & ONCE THROUGH \\
\hline $\begin{array}{l}\text { SILVER LAKE } \\
(\mathrm{MN})\end{array}$ & $\begin{array}{l}\text { ROCHESTER (MN) } \\
\text { PUB UTIL }\end{array}$ & $\mathrm{MN}$ & 16181 & 2008 & 1 & 8 & Standby & 1948 & COAL & FRESH & ONCE THROUGH \\
\hline $\begin{array}{l}\text { SILVER LAKE } \\
(\mathrm{MN})\end{array}$ & $\begin{array}{l}\text { ROCHESTER (MN) } \\
\text { PUB UTIL }\end{array}$ & $\mathrm{MN}$ & 16181 & 2008 & 4 & 54 & Operational & 1969 & COAL & FRESH & $\begin{array}{l}\text { COMBINATION (ONCE } \\
\text { THROUGH/HELPER TOWER) }\end{array}$ \\
\hline $\begin{array}{l}\text { SILVER LAKE } \\
(\mathrm{MN})\end{array}$ & $\begin{array}{l}\text { ROCHESTER (MN) } \\
\text { PUB UTIL }\end{array}$ & $\mathrm{MN}$ & 16181 & 2008 & 2 & 12 & Standby & 1953 & GAS & FRESH & ONCE THROUGH \\
\hline $\begin{array}{l}\text { SILVER LAKE } \\
\text { (MN) }\end{array}$ & $\begin{array}{l}\text { ROCHESTER (MN) } \\
\text { PUB UTIL }\end{array}$ & $\mathrm{MN}$ & 16181 & 2008 & 3 & 25 & Operational & 1962 & COAL & FRESH & $\begin{array}{l}\text { COMBINATION (ONCE } \\
\text { THROUGH/HELPER TOWER) }\end{array}$ \\
\hline SIOUX & UNION ELECTRIC CO & $\mathrm{MO}$ & 19436 & 2107 & 1 & 550 & Operational & 1967 & COAL & FRESH & ONCE THROUGH \\
\hline SIOUX & UNION ELECTRIC CO & $\mathrm{MO}$ & 19436 & 2107 & 2 & 550 & Operational & 1968 & COAL & FRESH & ONCE THROUGH \\
\hline $\begin{array}{l}\text { SOMERSET } \\
\text { (MA) }\end{array}$ & $\begin{array}{l}\text { MONTAUP } \\
\text { ELECTRIC CO }\end{array}$ & MA & 12833 & 1613 & 6 & 100 & Operational & 1959 & COAL & BRACKISH & ONCE THROUGH \\
\hline SOUTH BAY & $\begin{array}{l}\text { SAN DIEGO GAS \& } \\
\text { ELEC CO }\end{array}$ & $\mathrm{CA}$ & 16609 & 310 & 1 & 136 & Operational & 1960 & GAS & SALINE & ONCE THROUGH \\
\hline SOUTH BAY & $\begin{array}{l}\text { SAN DIEGO GAS \& } \\
\text { ELEC CO }\end{array}$ & $\mathrm{CA}$ & 16609 & 310 & 2 & 136 & Operational & 1962 & GAS & SALINE & ONCE THROUGH \\
\hline
\end{tabular}




\begin{tabular}{|c|c|c|c|c|c|c|c|c|c|c|c|}
\hline Plant Name & Utility Name & State & Utility/Pla & $n t / U n i$ & $I D$ & $M W$ & Status & Year & Fuel & Water & Cooling Systen \\
\hline SOUTH BAY & $\begin{array}{l}\text { SAN DIEGO GAS \& } \\
\text { ELEC CO }\end{array}$ & $\mathrm{CA}$ & 16609 & 310 & 3 & 202 & Operational & 1964 & GAS & SALINE & ONCE THROUGH \\
\hline SOUTH BAY & $\begin{array}{l}\text { SAN DIEGO GAS \& } \\
\text { ELEC CO }\end{array}$ & $\mathrm{CA}$ & 16609 & 310 & 4 & 240 & Operational & 1971 & GAS & SALINE & ONCE THROUGH \\
\hline SOUTHSIDE & $\begin{array}{l}\text { JACKSONVILLE } \\
\text { ELEC AUTH }\end{array}$ & $\mathrm{FL}$ & 9617 & 668 & 5 & 157 & Operational & 1964 & OIL & BRACKISH & ONCE THROUGH \\
\hline SOUTHSIDE & $\begin{array}{l}\text { JACKSONVILLE } \\
\text { ELEC AUTH }\end{array}$ & $\mathrm{FL}$ & 9617 & 668 & 4 & 75 & Operational & 1958 & GAS & BRACKISH & ONCE THROUGH \\
\hline SOUTHSIDE & $\begin{array}{l}\text { JACKSONVILLE } \\
\text { ELEC AUTH }\end{array}$ & $\mathrm{FL}$ & 9617 & 668 & 3 & 50 & Standby & 1955 & OIL & BRACKISH & ONCE THROUGH \\
\hline SPRINGDALE & $\begin{array}{l}\text { WEST PENN } \\
\text { POWER CO }\end{array}$ & PA & 20387 & 3182 & 8 & 141 & Standby & 1954 & OIL & FRESH & ONCE THROUGH \\
\hline SPRINGDALE & $\begin{array}{l}\text { WEST PENN } \\
\text { POWER CO }\end{array}$ & PA & 20387 & 3182 & 7 & 74.8 & Standby & 1945 & OIL & FRESH & ONCE THROUGH \\
\hline ST CLAIR & $\begin{array}{l}\text { DETROIT EDISON } \\
\text { CO }\end{array}$ & MI & 5109 & 1743 & 4 & 169 & Operational & 1954 & COAL & FRESH & ONCE THROUGH \\
\hline ST CLAIR & $\begin{array}{l}\text { DETROIT EDISON } \\
\text { CO }\end{array}$ & MI & 5109 & 1743 & 7 & 545 & Operational & 1969 & COAL & FRESH & ONCE THROUGH \\
\hline ST CLAIR & $\begin{array}{l}\text { DETROIT EDISON } \\
\text { CO }\end{array}$ & MI & 5109 & 1743 & 5 & 358 & Standby & 1959 & OIL & FRESH & ONCE THROUGH \\
\hline ST CLAIR & $\begin{array}{l}\text { DETROIT EDISON } \\
\text { CO }\end{array}$ & MI & 5109 & 1743 & 2 & 156 & Operational & 1953 & COAL & FRESH & ONCE THROUGH \\
\hline ST CLAIR & $\begin{array}{l}\text { DETROIT EDISON } \\
\text { CO }\end{array}$ & MI & 5109 & 1743 & 1 & 169 & Operational & 1953 & COAL & FRESH & ONCE THROUGH \\
\hline ST CLAIR & $\begin{array}{l}\text { DETROIT EDISON } \\
\text { CO }\end{array}$ & MI & 5109 & 1743 & 6 & 353 & Operational & 1961 & COAL & FRESH & ONCE THROUGH \\
\hline ST CLAIR & $\begin{array}{l}\text { DETROIT EDISON } \\
\text { CO }\end{array}$ & MI & 5109 & 1743 & 3 & 156 & Operational & 1954 & COAL & FRESH & ONCE THROUGH \\
\hline
\end{tabular}




\begin{tabular}{|c|c|c|c|c|c|c|c|c|c|c|c|}
\hline Plant Name & Utility Name & State & Utility/P & $n t / U n i$ & $I D$ & $\boldsymbol{M W}$ & Status & Year & Fuel & Water & Cooling System \\
\hline ST LUCIE & $\begin{array}{l}\text { FLORIDA POWER \& } \\
\text { LIGHT CO }\end{array}$ & $\mathrm{FL}$ & 6452 & 6045 & 1 & 850 & Operational & 1976 & UR & SALINE & ONCE THROUGH \\
\hline ST LUCIE & $\begin{array}{l}\text { FLORIDA POWER \& } \\
\text { LIGHT CO }\end{array}$ & $\mathrm{FL}$ & 6452 & 6045 & 2 & 850 & Operational & 1983 & UR & SALINE & ONCE THROUGH \\
\hline ST MARYS $(\mathrm{OH})$ & $\begin{array}{l}\text { ST MARYS MUN LT } \\
\text { \& POWER }\end{array}$ & $\mathrm{OH}$ & 17891 & 2942 & 4 & 2.5 & Standby & 1946 & COAL & FRESH & ONCE THROUGH \\
\hline ST MARYS $(\mathrm{OH})$ & $\begin{array}{l}\text { ST MARYS MUN LT } \\
\text { \& POWER }\end{array}$ & $\mathrm{OH}$ & 17891 & 2942 & 5 & 6 & Standby & 1957 & COAL & FRESH & ONCE THROUGH \\
\hline ST MARYS $(\mathrm{OH})$ & $\begin{array}{l}\text { ST MARYS MUN LT } \\
\text { \& POWER }\end{array}$ & $\mathrm{OH}$ & 17891 & 2942 & 6 & 10 & Operational & 1967 & COAL & FRESH & ONCE THROUGH \\
\hline STANTON (ND) & $\begin{array}{l}\text { UNITED POWER } \\
\text { ASSOCIATION }\end{array}$ & ND & 19514 & 2824 & 1 & 172 & Operational & 1967 & COAL & FRESH & ONCE THROUGH \\
\hline STANTON (ND) & $\begin{array}{l}\text { UNITED POWER } \\
\text { ASSOCIATION }\end{array}$ & ND & 19514 & 2824 & 10 & 60 & Operational & 1982 & COAL & FRESH & ONCE THROUGH \\
\hline STATE LINE & $\begin{array}{l}\text { COMMONWEALTH } \\
\text { EDISON (IN) }\end{array}$ & IN & 4111 & 981 & 3 & 225 & Operational & 1955 & COAL & FRESH & ONCE THROUGH \\
\hline STATE LINE & $\begin{array}{l}\text { COMMONWEALTH } \\
\text { EDISON (IN) }\end{array}$ & IN & 4111 & 981 & 4 & 389 & Operational & 1962 & COAL & FRESH & ONCE THROUGH \\
\hline STERLINGTON & $\begin{array}{l}\text { LOUISIANA POWER } \\
\& \text { LIGHT }\end{array}$ & LA & 11241 & 1404 & 6 & 225 & Standby & 1958 & GAS & FRESH & ONCE THROUGH \\
\hline STREETER & $\begin{array}{l}\text { CEDAR FALLS } \\
\text { UTILITIES }\end{array}$ & IA & 3203 & 1131 & 6 & 16.5 & Operational & 1963 & COAL & GROUND & ONCE THROUGH \\
\hline SUNBURY & $\begin{array}{l}\text { PENNSYLVANIA } \\
\text { POWER \& LT }\end{array}$ & PA & 14715 & 3152 & 1 & 75 & Operational & 1949 & COAL & FRESH & ONCE THROUGH \\
\hline SUNBURY & $\begin{array}{l}\text { PENNSYLVANIA } \\
\text { POWER \& LT }\end{array}$ & PA & 14715 & 3152 & 2 & 75 & Operational & 1949 & COAL & FRESH & ONCE THROUGH \\
\hline SUNBURY & $\begin{array}{l}\text { PENNSYLVANIA } \\
\text { POWER \& LT }\end{array}$ & PA & 14715 & 3152 & 3 & 104 & Operational & 1951 & COAL & FRESH & ONCE THROUGH \\
\hline
\end{tabular}




\begin{tabular}{|c|c|c|c|c|c|c|c|c|c|c|c|}
\hline Plant Name & Utility Name & State & Utility/Pl & $n t / U n i$ & $I D$ & $M W$ & Status & Year & Fuel & Water & Cooling System \\
\hline SUNBURY & $\begin{array}{l}\text { PENNSYLVANIA } \\
\text { POWER \& LT }\end{array}$ & PA & 14715 & 3152 & 4 & 156 & Operational & 1953 & COAL & FRESH & ONCE THROUGH \\
\hline SURRY & $\begin{array}{l}\text { VIRGINIA ELEC \& } \\
\text { POWER CO }\end{array}$ & VA & 19876 & 3806 & 1 & 848 & Operational & 1972 & UR & BRACKISH & ONCE THROUGH \\
\hline SURRY & $\begin{array}{l}\text { VIRGINIA ELEC \& } \\
\text { POWER CO }\end{array}$ & VA & 19876 & 3806 & 2 & 848 & Operational & 1973 & UR & BRACKISH & ONCE THROUGH \\
\hline $\begin{array}{l}\text { SUWANNEE } \\
\text { RIVER }\end{array}$ & $\begin{array}{l}\text { FLORIDA POWER } \\
\text { CORP }\end{array}$ & $\mathrm{FL}$ & 6455 & 638 & 3 & 75 & Operational & 1956 & OIL & FRESH & ONCE THROUGH \\
\hline $\begin{array}{l}\text { SUWANNEE } \\
\text { RIVER }\end{array}$ & $\begin{array}{l}\text { FLORIDA POWER } \\
\text { CORP }\end{array}$ & $\mathrm{FL}$ & 6455 & 638 & 1 & 34.5 & Operational & 1953 & OIL & FRESH & ONCE THROUGH \\
\hline $\begin{array}{l}\text { SUWANNEE } \\
\text { RIVER }\end{array}$ & $\begin{array}{l}\text { FLORIDA POWER } \\
\text { CORP }\end{array}$ & $\mathrm{FL}$ & 6455 & 638 & 2 & 37.5 & Operational & 1954 & OIL & FRESH & ONCE THROUGH \\
\hline SYL LASKIN & $\begin{array}{l}\text { MINNESOTA } \\
\text { POWER \& LIGHT }\end{array}$ & MN & 12647 & 1891 & 1 & 58.1 & Operational & 1953 & COAL & FRESH & ONCE THROUGH \\
\hline SYL LASKIN & $\begin{array}{l}\text { MINNESOTA } \\
\text { POWER \& LIGHT }\end{array}$ & MN & 12647 & 1891 & 2 & 58 & Operational & 1953 & COAL & FRESH & ONCE THROUGH \\
\hline TANGUISSON & $\begin{array}{l}\text { GUAM POWER } \\
\text { AUTHORITY }\end{array}$ & GU & 40428 & & 1 & 26.5 & Operational & 1971 & OIL & SALINE & ONCE THROUGH \\
\hline TANGUISSON & $\begin{array}{l}\text { GUAM POWER } \\
\text { AUTHORITY }\end{array}$ & GU & 40428 & & 2 & 26.5 & Operational & 1972 & OIL & SALINE & ONCE THROUGH \\
\hline $\begin{array}{l}\text { TANNERS } \\
\text { CREEK }\end{array}$ & $\begin{array}{l}\text { INDIANA MICHIGAN } \\
\text { POWER CO }\end{array}$ & IN & 9249 & 988 & 1 & 153 & Operational & 1951 & COAL & FRESH & ONCE THROUGH \\
\hline $\begin{array}{l}\text { TANNERS } \\
\text { CREEK }\end{array}$ & $\begin{array}{l}\text { INDIANA MICHIGAN } \\
\text { POWER CO }\end{array}$ & IN & 9249 & 988 & 2 & 153 & Operational & 1952 & COAL & FRESH & ONCE THROUGH \\
\hline $\begin{array}{l}\text { TANNERS } \\
\text { CREEK }\end{array}$ & $\begin{array}{l}\text { INDIANA MICHIGAN } \\
\text { POWER CO }\end{array}$ & IN & 9249 & 988 & 3 & 215 & Operational & 1954 & COAL & FRESH & ONCE THROUGH \\
\hline $\begin{array}{l}\text { TANNERS } \\
\text { CREEK }\end{array}$ & $\begin{array}{l}\text { INDIANA MICHIGAN } \\
\text { POWER CO }\end{array}$ & IN & 9249 & 988 & 4 & 580 & Operational & 1964 & COAL & FRESH & ONCE THROUGH \\
\hline
\end{tabular}




\begin{tabular}{|c|c|c|c|c|c|c|c|c|c|c|c|}
\hline Plant Name & Utility Name & State & Utility/Pl & $n t / U n i$ & $I D$ & $M W$ & Status & Year & Fuel & Water & Cooling System \\
\hline TECHE & $\begin{array}{l}\text { CENTRAL } \\
\text { LOUISIANA ELEC CO }\end{array}$ & LA & 3265 & 1400 & 3 & 349 & Operational & 1971 & GAS & BRACKISH & ONCE THROUGH \\
\hline TECHE & $\begin{array}{l}\text { CENTRAL } \\
\text { LOUISIANA ELEC CO }\end{array}$ & LA & 3265 & 1400 & 2 & 54.4 & Standby & 1956 & GAS & BRACKISH & ONCE THROUGH \\
\hline TECHE & $\begin{array}{l}\text { CENTRAL } \\
\text { LOUISIANA ELEC CO }\end{array}$ & LA & 3265 & 1400 & 1 & 25 & Standby & 1953 & GAS & BRACKISH & ONCE THROUGH \\
\hline TH ALLEN & $\begin{array}{l}\text { TENNESSEE } \\
\text { VALLEY AUTH }\end{array}$ & $\mathrm{TN}$ & 18642 & 3393 & 3 & 330 & Operational & 1959 & COAL & FRESH & ONCE THROUGH \\
\hline TH ALLEN & $\begin{array}{l}\text { TENNESSEE } \\
\text { VALLEY AUTH }\end{array}$ & $\mathrm{TN}$ & 18642 & 3393 & 1 & 330 & Operational & 1958 & COAL & FRESH & ONCE THROUGH \\
\hline TH ALLEN & $\begin{array}{l}\text { TENNESSEE } \\
\text { VALLEY AUTH }\end{array}$ & $\mathrm{TN}$ & 18642 & 3393 & 2 & 330 & Operational & 1959 & COAL & FRESH & ONCE THROUGH \\
\hline THIRD STREET & $\begin{array}{l}\text { CLARKSDALE PUB } \\
\text { UTIL COMM }\end{array}$ & MS & 3702 & 2059 & 5 & 7.5 & Standby & 1952 & GAS & GROUND & ONCE THROUGH \\
\hline THIRD STREET & $\begin{array}{l}\text { CLARKSDALE PUB } \\
\text { UTIL COMM }\end{array}$ & MS & 3702 & 2059 & 4 & 3.5 & Standby & 1947 & GAS & GROUND & ONCE THROUGH \\
\hline $\begin{array}{l}\text { TRENTON } \\
\text { CHANNEL }\end{array}$ & $\begin{array}{l}\text { DETROIT EDISON } \\
\text { CO }\end{array}$ & MI & 5109 & 1745 & 8 & 120 & Operational & 1950 & COAL & FRESH & ONCE THROUGH \\
\hline $\begin{array}{l}\text { TRENTON } \\
\text { CHANNEL }\end{array}$ & $\begin{array}{l}\text { DETROIT EDISON } \\
\text { CO }\end{array}$ & MI & 5109 & 1745 & 9 & 536 & Operational & 1968 & COAL & FRESH & ONCE THROUGH \\
\hline $\begin{array}{l}\text { TRENTON } \\
\text { CHANNEL }\end{array}$ & $\begin{array}{l}\text { DETROIT EDISON } \\
\text { CO }\end{array}$ & $\mathrm{Ml}$ & 5109 & 1745 & 7 & 120 & Operational & 1949 & COAL & FRESH & ONCE THROUGH \\
\hline TYRONE (KY) & $\begin{array}{l}\text { KENTUCKY } \\
\text { UTILITIES CO }\end{array}$ & KY & 10171 & 1361 & 3 & 75 & Operational & 1953 & COAL & FRESH & ONCE THROUGH \\
\hline TYRONE (KY) & $\begin{array}{l}\text { KENTUCKY } \\
\text { UTILITIES CO }\end{array}$ & KY & 10171 & 1361 & 1 & 31.3 & Standby & 1947 & OIL & FRESH & ONCE THROUGH \\
\hline TYRONE (KY) & $\begin{array}{l}\text { KENTUCKY } \\
\text { UTILITIES CO }\end{array}$ & KY & 10171 & 1361 & 2 & 31.3 & Standby & 1948 & OIL & FRESH & ONCE THROUGH \\
\hline
\end{tabular}




\begin{tabular}{|c|c|c|c|c|c|c|c|c|c|c|c|}
\hline Plant Name & Utility Name & State & Utility/Pl & $n t / U n i$ & $I D$ & $M W$ & Status & Year & Fuel & Water & Cooling System \\
\hline URQUHART & $\begin{array}{l}\text { SOUTH CAROLINA } \\
\text { ELEC \& GAS }\end{array}$ & SC & 17539 & 3295 & 3 & 100 & Operational & 1955 & COAL & FRESH & ONCE THROUGH \\
\hline URQUHART & $\begin{array}{l}\text { SOUTH CAROLINA } \\
\text { ELEC \& GAS }\end{array}$ & SC & 17539 & 3295 & 1 & 75 & Operational & 1953 & COAL & FRESH & ONCE THROUGH \\
\hline URQUHART & $\begin{array}{l}\text { SOUTH CAROLINA } \\
\text { ELEC \& GAS }\end{array}$ & SC & 17539 & 3295 & 2 & 75 & Operational & 1954 & COAL & FRESH & ONCE THROUGH \\
\hline VALLEY (WI) & $\begin{array}{l}\text { WISCONSIN ELEC } \\
\text { POWER CO }\end{array}$ & WI & 20847 & 4042 & 2 & 136 & Operational & 1969 & COAL & FRESH & ONCE THROUGH \\
\hline VALLEY (WI) & $\begin{array}{l}\text { WISCONSIN ELEC } \\
\text { POWER CO }\end{array}$ & WI & 20847 & 4042 & 1 & 136 & Operational & 1968 & COAL & FRESH & ONCE THROUGH \\
\hline $\begin{array}{l}\text { VENICE (IL) } \\
\text { TWO }\end{array}$ & UNION ELECTRIC CO & IL & 19436 & 913 & 5 & 98 & Standby & 1950 & GAS & FRESH & ONCE THROUGH \\
\hline $\begin{array}{l}\text { VENICE (IL) } \\
\text { TWO }\end{array}$ & UNION ELECTRIC CO & IL & 19436 & 913 & 6 & 100 & Standby & 1950 & GAS & FRESH & ONCE THROUGH \\
\hline $\begin{array}{l}\text { VENICE (IL) } \\
\text { TWO }\end{array}$ & UNION ELECTRIC CO & IL & 19436 & 913 & 4 & 98 & Standby & 1948 & GAS & FRESH & ONCE THROUGH \\
\hline $\begin{array}{l}\text { VENICE (IL) } \\
\text { TWO }\end{array}$ & UNION ELECTRIC CO & IL & 19436 & 913 & 3 & 98 & Standby & 1943 & GAS & FRESH & ONCE THROUGH \\
\hline $\begin{array}{l}\text { VENICE (IL) } \\
\text { TWO }\end{array}$ & UNION ELECTRIC CO & IL & 19436 & 913 & 2 & 40 & Standby & 1942 & GAS & FRESH & ONCE THROUGH \\
\hline $\begin{array}{l}\text { VENICE (IL) } \\
\text { TWO }\end{array}$ & UNION ELECTRIC CO & IL & 19436 & 913 & 1 & 40 & Standby & 1942 & GAS & FRESH & ONCE THROUGH \\
\hline $\begin{array}{l}\text { VERMONT } \\
\text { YANKEE }\end{array}$ & $\begin{array}{l}\text { VERMONT YANKEE } \\
\text { NUC POWER }\end{array}$ & VT & 19796 & 3751 & 1 & 540 & Operational & 1972 & UR & FRESH & $\begin{array}{l}\text { MIXED MODE (ONCE } \\
\text { THROUGH/COOLING TOWER) }\end{array}$ \\
\hline VERO BEACH & $\begin{array}{l}\text { VERO BEACH } \\
\text { MUNICIPAL UTIL }\end{array}$ & $\mathrm{FL}$ & 19804 & 693 & 1 & 12.5 & Standby & 1961 & GAS & BRACKISH & ONCE THROUGH \\
\hline VERO BEACH & $\begin{array}{l}\text { VERO BEACH } \\
\text { MUNICIPAL UTIL }\end{array}$ & $\mathrm{FL}$ & 19804 & 693 & 2 & 16.5 & Standby & 1964 & GAS & BRACKISH & ONCE THROUGH \\
\hline
\end{tabular}




\begin{tabular}{|c|c|c|c|c|c|c|c|c|c|c|c|}
\hline Plant Name & Utility Name & State & Utility/Pl & $n t / U n i$ & $I D$ & $M W$ & Status & Year & Fuel & Water & Cooling System \\
\hline VERO BEACH & $\begin{array}{l}\text { VERO BEACH } \\
\text { MUNICIPAL UTIL }\end{array}$ & $\mathrm{FL}$ & 19804 & 693 & 3 & 33 & Standby & 1971 & GAS & BRACKISH & ONCE THROUGH \\
\hline VICTORIA (TX) & $\begin{array}{l}\text { CENTRAL POWER \& } \\
\text { LIGHT CO }\end{array}$ & $\mathrm{TX}$ & 3278 & 3443 & 4 & 75 & Standby & 1955 & GAS & FRESH & $\begin{array}{l}\text { MIXED MODE (ONCE } \\
\text { THROUGH/COOLING TOWER) }\end{array}$ \\
\hline VICTORIA (TX) & $\begin{array}{l}\text { CENTRAL POWER \& } \\
\text { LIGHT CO }\end{array}$ & TX & 3278 & 3443 & 5 & 180 & Standby & 1963 & GAS & FRESH & $\begin{array}{l}\text { MIXED MODE (ONCE } \\
\text { THROUGH/COOLING TOWER) }\end{array}$ \\
\hline VIRGINIA & $\begin{array}{l}\text { VIRGINIA PUB UTIL } \\
\text { COMM }\end{array}$ & $\mathrm{MN}$ & 19883 & 2018 & 5 & 7.5 & Operational & 1954 & COAL & FRESH & ONCE THROUGH \\
\hline VIRGINIA & $\begin{array}{l}\text { VIRGINIA PUB UTIL } \\
\text { COMM }\end{array}$ & $\mathrm{MN}$ & 19883 & 2018 & 6 & 18.5 & Operational & 1971 & COAL & FRESH & ONCE THROUGH \\
\hline $\begin{array}{l}\text { WABASH } \\
\text { RIVER }\end{array}$ & PSI ENERGY INC & IN & 15470 & 1010 & 6 & 387 & Operational & 1968 & COAL & FRESH & ONCE THROUGH \\
\hline $\begin{array}{l}\text { WABASH } \\
\text { RIVER }\end{array}$ & PSI ENERGY INC & IN & 15470 & 1010 & 5 & 125 & Operational & 1956 & COAL & FRESH & ONCE THROUGH \\
\hline $\begin{array}{l}\text { WABASH } \\
\text { RIVER }\end{array}$ & PSI ENERGY INC & IN & 15470 & 1010 & 4 & 113 & Standby & 1955 & COAL & FRESH & ONCE THROUGH \\
\hline $\begin{array}{l}\text { WABASH } \\
\text { RIVER }\end{array}$ & PSI ENERGY INC & IN & 15470 & 1010 & 3 & 123 & Operational & 1954 & COAL & FRESH & ONCE THROUGH \\
\hline $\begin{array}{l}\text { WABASH } \\
\text { RIVER }\end{array}$ & PSI ENERGY INC & IN & 15470 & 1010 & 1 & 113 & Operational & 1953 & COAL & FRESH & ONCE THROUGH \\
\hline $\begin{array}{l}\text { WABASH } \\
\text { RIVER }\end{array}$ & PSI ENERGY INC & IN & 15470 & 1010 & 2 & 113 & Operational & 1953 & COAL & FRESH & ONCE THROUGH \\
\hline WAIAU & $\begin{array}{l}\text { HAWAIIAN } \\
\text { ELECTRIC CO }\end{array}$ & $\mathrm{HI}$ & 19547 & 766 & 6 & 54.4 & Operational & 1961 & OIL & SALINE & ONCE THROUGH \\
\hline WAIAU & $\begin{array}{l}\text { HAWAIIAN } \\
\text { ELECTRIC CO }\end{array}$ & $\mathrm{HI}$ & 19547 & 766 & 7 & 81.6 & Operational & 1966 & OIL & SALINE & ONCE THROUGH \\
\hline WAIAU & $\begin{array}{l}\text { HAWAIIAN } \\
\text { ELECTRIC CO }\end{array}$ & $\mathrm{HI}$ & 19547 & 766 & 8 & 81.6 & Operational & 1968 & OIL & SALINE & ONCE THROUGH \\
\hline
\end{tabular}




\begin{tabular}{|c|c|c|c|c|c|c|c|c|c|c|c|}
\hline Plant Name & Utility Name & State & Utility/Pl & $n t / U n i$ & $I D$ & $M W$ & Status & Year & Fuel & Water & Cooling System \\
\hline WAIAU & $\begin{array}{l}\text { HAWAIIAN } \\
\text { ELECTRIC CO }\end{array}$ & $\mathrm{HI}$ & 19547 & 766 & 3 & 50 & Operational & 1947 & OIL & SALINE & ONCE THROUGH \\
\hline WAIAU & $\begin{array}{l}\text { HAWAIIAN } \\
\text { ELECTRIC CO }\end{array}$ & $\mathrm{HI}$ & 19547 & 766 & 4 & 50 & Standby & 1950 & OIL & SALINE & ONCE THROUGH \\
\hline WAIAU & $\begin{array}{l}\text { HAWAIIAN } \\
\text { ELECTRIC CO }\end{array}$ & $\mathrm{HI}$ & 19547 & 766 & 5 & 54.4 & Operational & 1959 & OIL & SALINE & ONCE THROUGH \\
\hline WARREN (PA) & $\begin{array}{l}\text { PENNSYLVANIA } \\
\text { ELEC CO }\end{array}$ & PA & 14711 & 3132 & 1 & 42.3 & Operational & 1948 & COAL & FRESH & ONCE THROUGH \\
\hline WARREN (PA) & $\begin{array}{l}\text { PENNSYLVANIA } \\
\text { ELEC CO }\end{array}$ & PA & 14711 & 3132 & 2 & 42.3 & Operational & 1949 & COAL & FRESH & ONCE THROUGH \\
\hline WARRICK & $\begin{array}{l}\text { SOUTHERN INDIANA } \\
\text { GAS ELEC }\end{array}$ & IN & 17633 & 6705 & 4 & 323 & Operational & 1970 & COAL & FRESH & ONCE THROUGH \\
\hline WATEREE (SC) & $\begin{array}{l}\text { SOUTH CAROLINA } \\
\text { ELEC \& GAS }\end{array}$ & SC & 17539 & 3297 & 1 & 386 & Operational & 1970 & COAL & FRESH & $\begin{array}{l}\text { MIXED MODE (ONCE } \\
\text { THROUGH/COOLING TOWER) }\end{array}$ \\
\hline WATEREE (SC) & $\begin{array}{l}\text { SOUTH CAROLINA } \\
\text { ELEC \& GAS }\end{array}$ & SC & 17539 & 3297 & 2 & 386 & Operational & 1971 & COAL & FRESH & $\begin{array}{l}\text { MIXED MODE (ONCE } \\
\text { THROUGH/COOLING TOWER) }\end{array}$ \\
\hline WATERFORD & $\begin{array}{l}\text { LOUISIANA POWER } \\
\& \text { LIGHT }\end{array}$ & LA & 11241 & 8056 & 1 & 446 & Operational & 1975 & GAS & FRESH & ONCE THROUGH \\
\hline WATERFORD & $\begin{array}{l}\text { LOUISIANA POWER } \\
\& \text { LIGHT }\end{array}$ & LA & 11241 & 8056 & 2 & 446 & Operational & 1975 & GAS & FRESH & ONCE THROUGH \\
\hline WATERFORD & $\begin{array}{l}\text { LOUISIANA POWER } \\
\& \text { LIGHT }\end{array}$ & LA & 11241 & 8056 & 3 & 1200 & Operational & 1985 & UR & FRESH & ONCE THROUGH \\
\hline $\begin{array}{l}\text { WATTS BAR } \\
\text { COAL }\end{array}$ & $\begin{array}{l}\text { TENNESSEE } \\
\text { VALLEY AUTH }\end{array}$ & $\mathrm{TN}$ & 18642 & 3419 & 1 & 60 & Standby & 1942 & COAL & FRESH & ONCE THROUGH \\
\hline $\begin{array}{l}\text { WATTS BAR } \\
\text { COAL }\end{array}$ & $\begin{array}{l}\text { TENNESSEE } \\
\text { VALLEY AUTH }\end{array}$ & $\mathrm{TN}$ & 18642 & 3419 & 2 & 60 & Standby & 1942 & COAL & FRESH & ONCE THROUGH \\
\hline $\begin{array}{l}\text { WATTS BAR } \\
\text { COAL }\end{array}$ & $\begin{array}{l}\text { TENNESSEE } \\
\text { VALLEY AUTH }\end{array}$ & $\mathrm{TN}$ & 18642 & 3419 & 3 & 60 & Standby & 1943 & COAL & FRESH & ONCE THROUGH \\
\hline
\end{tabular}




\begin{tabular}{|c|c|c|c|c|c|c|c|c|c|c|c|}
\hline Plant Name & Utility Name & State & Utility/P & $n t / U n i$ & $I D$ & $M W$ & Status & Year & Fuel & Water & Cooling System \\
\hline $\begin{array}{l}\text { WATTS BAR } \\
\text { COAL }\end{array}$ & $\begin{array}{l}\text { TENNESSEE } \\
\text { VALLEY AUTH }\end{array}$ & $\mathrm{TN}$ & 18642 & 3419 & 4 & 60 & Standby & 1945 & COAL & FRESH & ONCE THROUGH \\
\hline WAUKEGAN & $\begin{array}{l}\text { COMMONWEALTH } \\
\text { EDISON CO }\end{array}$ & $\mathrm{IL}$ & 4110 & 883 & 6 & 121 & Operational & 1952 & COAL & FRESH & ONCE THROUGH \\
\hline WAUKEGAN & $\begin{array}{l}\text { COMMONWEALTH } \\
\text { EDISON CO }\end{array}$ & IL & 4110 & 883 & 7 & 326 & Operational & 1958 & COAL & FRESH & ONCE THROUGH \\
\hline WAUKEGAN & $\begin{array}{l}\text { COMMONWEALTH } \\
\text { EDISON CO }\end{array}$ & $\mathrm{IL}$ & 4110 & 883 & 8 & 355 & Operational & 1962 & COAL & FRESH & ONCE THROUGH \\
\hline WC BECKJORD & $\begin{array}{l}\text { CINCINNATI GAS \& } \\
\text { ELEC CO }\end{array}$ & $\mathrm{OH}$ & 3542 & 2830 & 4 & 163 & Operational & 1958 & COAL & FRESH & ONCE THROUGH \\
\hline WC BECKJORD & $\begin{array}{l}\text { CINCINNATI GAS \& } \\
\text { ELEC CO }\end{array}$ & $\mathrm{OH}$ & 3542 & 2830 & 5 & 245 & Operational & 1962 & COAL & FRESH & ONCE THROUGH \\
\hline WC BECKJORD & $\begin{array}{l}\text { CINCINNATI GAS \& } \\
\text { ELEC CO }\end{array}$ & $\mathrm{OH}$ & 3542 & 2830 & 1 & 115 & Operational & 1952 & COAL & FRESH & ONCE THROUGH \\
\hline WC BECKJORD & $\begin{array}{l}\text { CINCINNATI GAS \& } \\
\text { ELEC CO }\end{array}$ & $\mathrm{OH}$ & 3542 & 2830 & 2 & 113 & Operational & 1953 & COAL & FRESH & ONCE THROUGH \\
\hline WC BECKJORD & $\begin{array}{l}\text { CINCINNATI GAS \& } \\
\text { ELEC CO }\end{array}$ & $\mathrm{OH}$ & 3542 & 2830 & 6 & 461 & Operational & 1969 & COAL & FRESH & ONCE THROUGH \\
\hline WC BECKJORD & $\begin{array}{l}\text { CINCINNATI GAS \& } \\
\text { ELEC CO }\end{array}$ & $\mathrm{OH}$ & 3542 & 2830 & 3 & 125 & Operational & 1954 & COAL & FRESH & ONCE THROUGH \\
\hline WEBSTER (TX) & $\begin{array}{l}\text { HOUSTON } \\
\text { LIGHTING \& POWER }\end{array}$ & TX & 8901 & 3471 & 3 & 410 & Operational & 1965 & GAS & BRACKISH & ONCE THROUGH \\
\hline WERNER & $\begin{array}{l}\text { JERSEY CENT } \\
\text { POWER \& LIGHT }\end{array}$ & NJ & 9726 & 2385 & 4 & 60 & Operational & 1953 & OIL & BRACKISH & ONCE THROUGH \\
\hline $\begin{array}{l}\text { WEST } \\
\text { SPRINGFIELD }\end{array}$ & $\begin{array}{l}\text { WESTERN MASS } \\
\text { ELEC CO }\end{array}$ & MA & 20455 & 1642 & 3 & 114 & Operational & 1957 & GAS & FRESH & ONCE THROUGH \\
\hline WESTON (WI) & $\begin{array}{l}\text { WISCONSIN PUBLIC } \\
\text { SERVICE }\end{array}$ & WI & 20860 & 4078 & 1 & 60 & Operational & 1954 & COAL & FRESH & ONCE THROUGH \\
\hline
\end{tabular}




\begin{tabular}{|c|c|c|c|c|c|c|c|c|c|c|c|}
\hline Plant Name & Utility Name & State & Utility/Pl & $n t / U n$ & $I D$ & $M W$ & Status & Year & Fuel & Water & Cooling Systen \\
\hline WESTON (WI) & $\begin{array}{l}\text { WISCONSIN PUBLIC } \\
\text { SERVICE }\end{array}$ & WI & 20860 & 4078 & 2 & 75 & Operational & 1960 & COAL & FRESH & ONCE THROUGH \\
\hline WH HILL & $\begin{array}{l}\text { HAWAII ELEC LIGHT } \\
\text { CO }\end{array}$ & $\mathrm{HI}$ & 8287 & 772 & 5 & 14.1 & Operational & 1965 & OIL & GROUND & ONCE THROUGH \\
\hline WH HILL & $\begin{array}{l}\text { HAWAII ELEC LIGHT } \\
\text { CO }\end{array}$ & $\mathrm{HI}$ & 8287 & 772 & 6 & 23 & Operational & 1974 & OIL & GROUND & ONCE THROUGH \\
\hline WH SAMMIS & OHIO EDISON CO & $\mathrm{OH}$ & 13998 & 2866 & 6 & 623 & Operational & 1969 & COAL & FRESH & ONCE THROUGH \\
\hline WH SAMMIS & OHIO EDISON CO & $\mathrm{OH}$ & 13998 & 2866 & 7 & 623 & Operational & 1971 & COAL & FRESH & ONCE THROUGH \\
\hline WH SAMMIS & OHIO EDISON CO & $\mathrm{OH}$ & 13998 & 2866 & 5 & 318 & Operational & 1967 & COAL & FRESH & ONCE THROUGH \\
\hline WH SAMMIS & OHIO EDISON CO & $\mathrm{OH}$ & 13998 & 2866 & 4 & 185 & Operational & 1962 & COAL & FRESH & ONCE THROUGH \\
\hline WH SAMMIS & OHIO EDISON CO & $\mathrm{OH}$ & 13998 & 2866 & 3 & 185 & Operational & 1961 & COAL & FRESH & ONCE THROUGH \\
\hline WH SAMMIS & OHIO EDISON CO & $\mathrm{OH}$ & 13998 & 2866 & 2 & 185 & Operational & 1960 & COAL & FRESH & ONCE THROUGH \\
\hline WH SAMMIS & OHIO EDISON CO & $\mathrm{OH}$ & 13998 & 2866 & 1 & 185 & Operational & 1959 & COAL & FRESH & ONCE THROUGH \\
\hline $\begin{array}{l}\text { WIDOWS } \\
\text { CREEK }\end{array}$ & $\begin{array}{l}\text { TENNESSEE } \\
\text { VALLEY AUTH }\end{array}$ & $\mathrm{AL}$ & 18642 & 50 & 5 & 141 & Operational & 1954 & COAL & FRESH & ONCE THROUGH \\
\hline $\begin{array}{l}\text { WIDOWS } \\
\text { CREEK }\end{array}$ & $\begin{array}{l}\text { TENNESSEE } \\
\text { VALLEY AUTH }\end{array}$ & $A L$ & 18642 & 50 & 8 & 550 & Operational & 1965 & COAL & FRESH & ONCE THROUGH \\
\hline $\begin{array}{l}\text { WIDOWS } \\
\text { CREEK }\end{array}$ & $\begin{array}{l}\text { TENNESSEE } \\
\text { VALLEY AUTH }\end{array}$ & $\mathrm{AL}$ & 18642 & 50 & 7 & 575 & Operational & 1961 & COAL & FRESH & ONCE THROUGH \\
\hline $\begin{array}{l}\text { WIDOWS } \\
\text { CREEK }\end{array}$ & $\begin{array}{l}\text { TENNESSEE } \\
\text { VALLEY AUTH }\end{array}$ & $\mathrm{AL}$ & 18642 & 50 & 6 & 141 & Operational & 1954 & COAL & FRESH & ONCE THROUGH \\
\hline
\end{tabular}




\begin{tabular}{|c|c|c|c|c|c|c|c|c|c|c|c|}
\hline Plant Name & Utility Name & State & Utility/Pl & $n t / U n i$ & $I D$ & $M W$ & Status & Year & Fuel & Water & Cooling System \\
\hline $\begin{array}{l}\text { WIDOWS } \\
\text { CREEK }\end{array}$ & $\begin{array}{l}\text { TENNESSEE } \\
\text { VALLEY AUTH }\end{array}$ & $A L$ & 18642 & 50 & 3 & 141 & Operational & 1952 & COAL & FRESH & ONCE THROUGH \\
\hline $\begin{array}{l}\text { WIDOWS } \\
\text { CREEK }\end{array}$ & $\begin{array}{l}\text { TENNESSEE } \\
\text { VALLEY AUTH }\end{array}$ & $\mathrm{AL}$ & 18642 & 50 & 2 & 141 & Operational & 1952 & COAL & FRESH & ONCE THROUGH \\
\hline $\begin{array}{l}\text { WIDOWS } \\
\text { CREEK }\end{array}$ & $\begin{array}{l}\text { TENNESSEE } \\
\text { VALLEY AUTH }\end{array}$ & $\mathrm{AL}$ & 18642 & 50 & 1 & 141 & Operational & 1952 & COAL & FRESH & ONCE THROUGH \\
\hline $\begin{array}{l}\text { WIDOWS } \\
\text { CREEK }\end{array}$ & $\begin{array}{l}\text { TENNESSEE } \\
\text { VALLEY AUTH }\end{array}$ & $\mathrm{AL}$ & 18642 & 50 & 4 & 141 & Operational & 1953 & COAL & FRESH & ONCE THROUGH \\
\hline WILL COUNTY & $\begin{array}{l}\text { COMMONWEALTH } \\
\text { EDISON CO }\end{array}$ & IL & 4110 & 884 & 3 & 299 & Operational & 1957 & COAL & FRESH & ONCE THROUGH \\
\hline WILL COUNTY & $\begin{array}{l}\text { COMMONWEALTH } \\
\text { EDISON CO }\end{array}$ & IL & 4110 & 884 & 4 & 598 & Operational & 1963 & COAL & FRESH & ONCE THROUGH \\
\hline WILL COUNTY & $\begin{array}{l}\text { COMMONWEALTH } \\
\text { EDISON CO }\end{array}$ & IL & 4110 & 884 & 2 & 184 & Operational & 1955 & COAL & FRESH & ONCE THROUGH \\
\hline WILL COUNTY & $\begin{array}{l}\text { COMMONWEALTH } \\
\text { EDISON CO }\end{array}$ & IL & 4110 & 884 & 1 & 188 & Operational & 1955 & COAL & FRESH & ONCE THROUGH \\
\hline WILLAMETTE & $\begin{array}{l}\text { EUGENE WATER \& } \\
\text { ELEC BD }\end{array}$ & OR & 6022 & & 2 & 7.5 & Standby & 1941 & WOOD & FRESH & ONCE THROUGH \\
\hline WILLAMETTE & $\begin{array}{l}\text { EUGENE WATER \& } \\
\text { ELEC BD }\end{array}$ & OR & 6022 & & 3 & 11.5 & Operational & 1950 & WOOD & FRESH & ONCE THROUGH \\
\hline WILLAMETTE & $\begin{array}{l}\text { EUGENE WATER \& } \\
\text { ELEC BD }\end{array}$ & OR & 6022 & & 1 & 6 & Standby & 1931 & WOOD & FRESH & ONCE THROUGH \\
\hline WILLOW GLEN & $\begin{array}{l}\text { GULF STATES } \\
\text { UTILITIES }\end{array}$ & LA & 7806 & 1394 & 1 & 163 & Operational & 1960 & GAS & FRESH & ONCE THROUGH \\
\hline WILLOW GLEN & $\begin{array}{l}\text { GULF STATES } \\
\text { UTILITIES }\end{array}$ & LA & 7806 & 1394 & 5 & 592 & Operational & 1976 & GAS & FRESH & ONCE THROUGH \\
\hline WILLOW GLEN & $\begin{array}{l}\text { GULF STATES } \\
\text { UTILITIES }\end{array}$ & LA & 7806 & 1394 & 4 & 592 & Operational & 1973 & GAS & FRESH & ONCE THROUGH \\
\hline
\end{tabular}




\begin{tabular}{|c|c|c|c|c|c|c|c|c|c|c|c|}
\hline Plant Name & Utility Name & State & Utility/Pl & $t /$ Uni & $I D$ & $M W$ & Status & Year & Fuel & Water & Cooling System \\
\hline WILLOW GLEN & $\begin{array}{l}\text { GULF STATES } \\
\text { UTILITIES }\end{array}$ & LA & 7806 & 1394 & 2 & 239 & Operational & 1964 & GAS & FRESH & ONCE THROUGH \\
\hline WILLOW GLEN & $\begin{array}{l}\text { GULF STATES } \\
\text { UTILITIES }\end{array}$ & LA & 7806 & 1394 & 3 & 592 & Standby & 1968 & GAS & FRESH & ONCE THROUGH \\
\hline $\begin{array}{l}\text { WILLOW } \\
\text { ISLAND }\end{array}$ & $\begin{array}{l}\text { MONONGAHELA } \\
\text { POWER CO }\end{array}$ & WV & 12796 & 3946 & 1 & 50 & Operational & 1949 & COAL & FRESH & ONCE THROUGH \\
\hline $\begin{array}{l}\text { WILLOW } \\
\text { ISLAND }\end{array}$ & $\begin{array}{l}\text { MONONGAHELA } \\
\text { POWER CO }\end{array}$ & WV & 12796 & 3946 & 2 & 163 & Operational & 1960 & COAL & FRESH & ONCE THROUGH \\
\hline WILMARTH & $\begin{array}{l}\text { NORTHERN STATES } \\
\text { POWER CO }\end{array}$ & MN & 13781 & 1934 & 1 & 12.5 & Operational & 1948 & RDF & FRESH & ONCE THROUGH \\
\hline WILMARTH & $\begin{array}{l}\text { NORTHERN STATES } \\
\text { POWER CO }\end{array}$ & MN & 13781 & 1934 & 2 & 12.5 & Operational & 1951 & RDF & FRESH & ONCE THROUGH \\
\hline WINFIELD (KS) & $\begin{array}{l}\text { WINFIELD MUN UTIL } \\
\text { COMM }\end{array}$ & KS & 20813 & 1332 & 2 & 5 & Standby & 1952 & GAS & FRESH & ONCE THROUGH \\
\hline WINFIELD (KS) & $\begin{array}{l}\text { WINFIELD MUN UTIL } \\
\text { COMM }\end{array}$ & $\mathrm{KS}$ & 20813 & 1332 & 1 & 10 & Operational & 1957 & GAS & FRESH & ONCE THROUGH \\
\hline WINNETKA & $\begin{array}{l}\text { WINNETKA ELEC } \\
\text { SYSTEM }\end{array}$ & IL & 20824 & 972 & 7 & 10 & Operational & 1964 & GAS & FRESH & ONCE THROUGH \\
\hline WINNETKA & $\begin{array}{l}\text { WINNETKA ELEC } \\
\text { SYSTEM }\end{array}$ & IL & 20824 & 972 & 4 & 7.5 & Operational & 1958 & GAS & FRESH & ONCE THROUGH \\
\hline WINNETKA & $\begin{array}{l}\text { WINNETKA ELEC } \\
\text { SYSTEM }\end{array}$ & IL & 20824 & 972 & 6 & 5 & Standby & 1948 & COAL & FRESH & ONCE THROUGH \\
\hline WN CLARK & $\begin{array}{l}\text { WESTPLAINS } \\
\text { ENERGY }\end{array}$ & $\mathrm{CO}$ & 20391 & 462 & 1 & 16.5 & Operational & 1955 & COAL & FRESH & $\begin{array}{l}\text { COMBINATION (ONCE } \\
\text { THROUGH/HELPER TOWER) }\end{array}$ \\
\hline WN CLARK & $\begin{array}{l}\text { WESTPLAINS } \\
\text { ENERGY }\end{array}$ & $\mathrm{CO}$ & 20391 & 462 & 2 & 22 & Operational & 1959 & COAL & FRESH & $\begin{array}{l}\text { COMBINATION (ONCE } \\
\text { THROUGH/HELPER TOWER) }\end{array}$ \\
\hline $\begin{array}{l}\text { WOOD RIVER } \\
\text { (IL) }\end{array}$ & ILLINOIS POWER CO & IL & 9208 & 898 & 1 & 50 & Standby & 1949 & GAS & FRESH & ONCE THROUGH \\
\hline
\end{tabular}




\begin{tabular}{|c|c|c|c|c|c|c|c|c|c|c|c|}
\hline Plant Name & Utility Name & State & Utility/Pl & t/Uni & $I D$ & $M W$ & Status & Year & Fuel & Water & Cooling System \\
\hline $\begin{array}{l}\text { WOOD RIVER } \\
\text { (IL) }\end{array}$ & ILLINOIS POWER CO & IL & 9208 & 898 & 2 & 50 & Standby & 1949 & GAS & FRESH & ONCE THROUGH \\
\hline $\begin{array}{l}\text { WOOD RIVER } \\
\text { (IL) }\end{array}$ & ILLINOIS POWER CO & IL & 9208 & 898 & 3 & 50 & Standby & 1950 & GAS & FRESH & ONCE THROUGH \\
\hline $\begin{array}{l}\text { WOOD RIVER } \\
\text { (IL) }\end{array}$ & ILLINOIS POWER CO & IL & 9208 & 898 & 4 & 113 & Operational & 1954 & COAL & FRESH & ONCE THROUGH \\
\hline $\begin{array}{l}\text { WOOD RIVER } \\
\text { (IL) }\end{array}$ & ILLINOIS POWER CO & IL & 9208 & 898 & 5 & 388 & Operational & 1964 & COAL & FRESH & ONCE THROUGH \\
\hline WYANDOTTE & $\begin{array}{l}\text { WYANDOTTE MUNI } \\
\text { SER COMM }\end{array}$ & $\mathrm{Ml}$ & 21048 & 1866 & 5 & 22 & Operational & 1958 & COAL & FRESH & ONCE THROUGH \\
\hline WYMAN & $\begin{array}{l}\text { CENTRAL MAINE } \\
\text { POWER CO }\end{array}$ & ME & 3266 & 1507 & 1 & 50 & Operational & 1957 & OIL & SALINE & ONCE THROUGH \\
\hline WYMAN & $\begin{array}{l}\text { CENTRAL MAINE } \\
\text { POWER CO }\end{array}$ & ME & 3266 & 1507 & 2 & 50 & Operational & 1958 & OIL & SALINE & ONCE THROUGH \\
\hline WYMAN & $\begin{array}{l}\text { CENTRAL MAINE } \\
\text { POWER CO }\end{array}$ & ME & 3266 & 1507 & 3 & 114 & Operational & 1965 & OIL & SALINE & ONCE THROUGH \\
\hline WYMAN & $\begin{array}{l}\text { CENTRAL MAINE } \\
\text { POWER CO }\end{array}$ & ME & 3266 & 1507 & 4 & 632 & Operational & 1978 & OIL & SALINE & ONCE THROUGH \\
\hline YATES & $\begin{array}{l}\text { GEORGIA POWER } \\
\text { CO }\end{array}$ & GA & 7140 & 728 & 5 & 156 & Operational & 1958 & COAL & FRESH & ONCE THROUGH \\
\hline YATES & $\begin{array}{l}\text { GEORGIA POWER } \\
\text { CO }\end{array}$ & GA & 7140 & 728 & 4 & 156 & Operational & 1957 & COAL & FRESH & ONCE THROUGH \\
\hline YATES & $\begin{array}{l}\text { GEORGIA POWER } \\
\text { CO }\end{array}$ & GA & 7140 & 728 & 3 & 123 & Operational & 1952 & COAL & FRESH & ONCE THROUGH \\
\hline YATES & $\begin{array}{l}\text { GEORGIA POWER } \\
\text { CO }\end{array}$ & GA & 7140 & 728 & 1 & 123 & Operational & 1950 & COAL & FRESH & ONCE THROUGH \\
\hline YATES & $\begin{array}{l}\text { GEORGIA POWER } \\
\text { CO }\end{array}$ & GA & 7140 & 728 & 2 & 123 & Operational & 1950 & COAL & FRESH & ONCE THROUGH \\
\hline
\end{tabular}




\begin{tabular}{|c|c|c|c|c|c|c|c|c|c|c|c|}
\hline Plant Name & Utility Name & State & Utility/P & $t / U n i$ & $D$ & $M W$ & Status & Year & Fuel & Water & Cooling System \\
\hline YAZOO CITY & $\begin{array}{l}\text { YAZOO CITY PUB } \\
\text { SER COMM }\end{array}$ & MS & 21095 & 2067 & 1 & 5 & Standby & 1945 & GAS & GROUND & ONCE THROUGH \\
\hline YORKTOWN & $\begin{array}{l}\text { VIRGINIA ELEC \& } \\
\text { POWER CO }\end{array}$ & VA & 19876 & 3809 & 1 & 188 & Operational & 1957 & COAL & BRACKISH & ONCE THROUGH \\
\hline YORKTOWN & $\begin{array}{l}\text { VIRGINIA ELEC \& } \\
\text { POWER CO }\end{array}$ & VA & 19876 & 3809 & 2 & 188 & Operational & 1958 & COAL & BRACKISH & ONCE THROUGH \\
\hline YORKTOWN & $\begin{array}{l}\text { VIRGINIA ELEC \& } \\
\text { POWER CO }\end{array}$ & VA & 19876 & 3809 & 3 & 882 & Operational & 1974 & OIL & BRACKISH & ONCE THROUGH \\
\hline ZION & $\begin{array}{l}\text { COMMONWEALTH } \\
\text { EDISON CO }\end{array}$ & IL & 4110 & 885 & 1 & 1085 & Operational & 1973 & UR & FRESH & ONCE THROUGH \\
\hline ZION & $\begin{array}{l}\text { COMMONWEALTH } \\
\text { EDISON CO }\end{array}$ & IL & 4110 & 885 & 2 & 1085 & Operational & 1974 & UR & FRESH & ONCE THROUGH \\
\hline ZUNI & $\begin{array}{l}\text { PUBLIC SERVICE } \\
\text { COLORADO }\end{array}$ & $\mathrm{CO}$ & 15466 & 478 & 1 & 35 & Operational & 1948 & GAS & FRESH & $\begin{array}{l}\text { COMBINATION (ONCE } \\
\text { THROUGH/HELPER TOWER) }\end{array}$ \\
\hline
\end{tabular}


Appendix 2 - "Cooling Water Use Patterns at U.S. Nonutility Electric Generating Stations" (manuscript submitted for publication in Environmental Science \& Policy, June 1999) 


\title{
Cooling Water Use Patterns at U.S. Nonutility Electric Generating Facilities
}

\author{
John A. Veil - Argonne National Laboratory \\ Markus G. Puder - Argonne National Laboratory \\ Debra J. Littleton - U.S. Department of Energy \\ David O. Moses - U.S. Department of Energy
}

\begin{abstract}
Cooling water is used by many industrial facilities. The largest user of cooling water is the electric power industry. Data pertaining to cooling water use patterns at utility plants are readily available; however, no information has been assembled for cooling water use at electric power generating facilities owned or operated by entities other than utilities (nonutilities). This paper presents cooling water use data from two subsets of the nonutility sector and focuses on plants using once-through cooling systems. The first subset includes 123 nonutility plants that each generate at least $150 \mathrm{MW}$ of power. Collectively, they represent $41,494 \mathrm{MW}$ of generating capacity, or about $56 \%$ of the total generating capacity. Approximately $17 \%$ of the installations within that subset utilize once-through cooling water. The second subset includes 58 waste-toenergy facilities, which individually produce less than $80 \mathrm{MW}$ but collectively generate about $2,200 \mathrm{MW}$. Only $11 \%$ of this subset of plants uses once-through cooling. The total 15,372 MW generated by once-through nonutilities is equivalent to only $6 \%$ of the $258,906 \mathrm{MW}$ generated by utilities utilizing once-through cooling. This share may, from a national perspective, appear relatively insignificant. However, in some states, the nonutility once-through total is equivalent to a more significant percentage of the utility once-through total.
\end{abstract}

\section{Introduction}

\section{I.I The U.S. Electric Power Industry}

The U.S. Department of Energy (DOE) Energy Information Administration (EIA) compiles, organizes, and publishes statistical data pertaining to various aspects of power production. For 1997, EIA (1998a) reports that U.S. generators produced 778,513 megawatts (MW) of electricity. Utilities produced $711,889 \mathrm{MW}$, whereas $74,021 \mathrm{MW}$ was generated by nonutility sources. EIA notes that its methodology does not permit direct summation of the utility and nonutility totals.

The relative proportions of utility and nonutility generating capacity sources will shift over time. The impending deregulation of the electric power industry and other market forces have prompted several utilities to divest themselves of generating capacity and to provide only power distribution services. For example, in early 1999, the Potomac Electric Power Company, based in Washington, D.C., announced its intention to sell its generating plants over the next few years. If the ultimate purchaser of those plants is a nonutility, nearly 6,000 MW of capacity would move from the utility total to the nonutility bracket.

Presently, over 3,250 U.S. electric utilities, including investor-owned utilities, publicly owned utilities, cooperative electric utilities, federal electric utilities, and power marketers, operate in 
the United States. More than $75 \%$ of U.S. electricity is supplied by investor-owned utilities (EIA 1998a). Fossil-fuel and nuclear steam electric plants generate nearly $545,000 \mathrm{MW}$. Gas turbines and internal combustion systems produce 65,000 MW. Hydroelectric and pumped storage systems generate $95,000 \mathrm{MW}$. Renewable sources produce about $2 \mathrm{MW}$. As a result of independent rounding, the sum of these generation rates does not equal the total for utilities provided above (EIA 1998b).

The Edison Electric Institute (EEI) Power Statistics Data Base estimates the installed U.S. steam electric generating capacity at 583,596 MW (EEI 1996). The discrepancy between the EIA and the EEI figures is attributable to differences in the ways in which data are obtained and updated. The EIA data are obtained through annual written questionnaires. The EEI data are developed by using the EIA data as a starting point. They were updated and revised regularly by contact with utilities through 1996. Since 1996, the EEI data have not been updated. The remainder of this paper uses EEI data for utilities because they provide more details pertaining to cooling water usage.

Approximately 2,000 U.S. nonutility power producers, including cogenerator qualified facilities, small power producers, exempt wholesale generators, cogenerator nonqualifying facilities, and independent power producers, operate in the United States. About $60 \%$ of nonutility capacity is generated by cogenerator qualified facilities. Some nonutilities sell their electricity to utilities, while others use it for their own internal consumption. Industrial plants that produce their own power to satisfy their huge electricity needs (e.g., iron and steel mills, paper mills) are considered nonutility generators.

\subsection{Cooling Water Use}

Water is used in many industrial applications to cool machinery or to condense steam. The largest industrial user of cooling water is the steam electric power industry. More than $75 \%$ of the utility-derived power in the United States is generated through the steam electric process. At nuclear and fossil-fuel power plants, electricity is produced by heating purified water to create steam. The steam is used to run turbines, which drive the generators that produce electricity. After leaving the turbines, the steam passes through a condenser, which has multiple tubes and a large surface area. A large volume of cool water circulates through the tubes, absorbing heat from the steam. As the steam cools and condenses, the temperature of the cooling water rises.

Most power plants use either once-through cooling or closed-cycle cooling. Once-through cooling systems withdraw large volumes of water from a river, lake, estuary, or ocean. The water is pumped through the condenser and finally returned to the same or a nearby water body. Closed-cycle cooling systems receive their cooling water from and return it to a cooling tower and basin, cooling pond, or cooling lake.

Table 1 (based on EEI 1996) shows the distribution of U.S. steam electric utility capacity by type of cooling system. Closed-cycle systems cool about $50 \%$ of the capacity, whereas once-through systems account for $44 \%$. The remainder is shared by systems utilizing both closed-cycle and once-through components ("combination") or switching seasonally between closed-cycle and once-through cooling ("mixed mode"). 
Table 2 (based on EEI 1996) provides information pertaining to the source of the cooling water used by utilities. Nearly half of the U.S. steam electric utility capacity utilizes cooling water withdrawn from rivers. Lakes, estuaries, and oceans supply cooling water for $27 \%, 11 \%$, and $9 \%$ of the capacity, respectively. The remaining $7 \%$ comes from a variety of other sources, including groundwater, tap water, and others.

\section{A Need for Nonutility Cooling Water Data}

Section 316(b) of the Clean Water Act, enacted by Congress in 1972, addresses withdrawal of cooling water from surface water bodies, as follows:

Any standard established pursuant to section 301 or section 306 of this Act and applicable to a point source shall require that the location, design, construction, and capacity of cooling water intake structures reflect the best technology available for minimizing adverse environmental impact.

In 1976, the U.S. Environmental Protection Agency (EPA) promulgated final $\$ 316(\mathrm{~b})$ regulations (April 26, 1976; 41 FR 17387). However, those regulations were successfully challenged by a group of 58 utilities [Appalachian Power Co. v. Train, 10 ERC $1965\left(4^{\text {th }}\right.$ Cir. 1977)]. In 1979, EPA formally withdrew its $§ 316$ (b) regulations (June 1979; 44 FR 32956). As a consequence of the vacuum created by the absence of federal regulations, states adopted their own cooling water intake regulations to implement the $\$ 316(\mathrm{~b})$ requirements. The broad statutory language facilitated widely differing interpretations by the states. Some adopted comprehensive programs, whereas others imposed less rigorous requirements.

In the mid-1990s, a coalition of environmental groups, headed by the Hudson Riverkeeper, filed suit against EPA over failure to repromulgate $\$ 316(\mathrm{~b})$ regulations [Cronin, et al. v. Reilly, 93 Civ. 0314 (AGS)]. On October 10, 1995, the U.S. District Court, Southern District of New York, entered a Consent Decree between the parties, directing EPA to regulate cooling water intake structures within 7 years. Under the Consent Decree, EPA agreed to propose regulations by June 1999 and promulgate a final rule by 2001 .

As a precursor to formal regulations, EPA is currently developing a draft framework governing cooling water intake structures. Determining the universe of cooling water users that may potentially be affected by a national $\$ 316($ b) regulation plays a crucial role in the regulatory process. Not every generating unit may be affected by the $\S 316(b)$ regulations. Some plants use closed-cycle cooling systems and other units generate power without a steam cycle, and therefore do not require a water-based cooling system. Consequently, EPA will probably inventory U.S. power plants using once-through cooling systems. However, cooling water use patterns at nonutilities cannot be readily extracted from existing information sources. The EEI Power Statistics Data Base includes only utilities and the EIA nonutility database does not address cooling water use.

DOE's mission includes monitoring actions that could either dramatically increase the cost of electricity to consumers or disrupt a reliable electricity supply for any parts of the country. DOE 
commissioned Argonne National Laboratory to obtain information that could be used to analyze the potential economic and reliability impacts of future $\$ 316(b)$ regulations (Veil 1999), including new data pertaining to cooling water use patterns at nonutilities. After describing the methodology for data collection, the paper presents and analyzes the data thus obtained.

\section{Methodology}

EIA (1998c) provides a list of approximately 2,000 U.S. nonutility power generators with at least $1 \mathrm{MW}$ capacity and includes information specifying the plant's name and owner, the state in which the plant is located, and the nameplate capacity of each plant. The size of these nonutility facilities ranges from I MW to $1849 \mathrm{MW}$. The EIA does not collect cooling water usage information from nonutilities. Neither do nonutility trade or industry associations maintain cooling water information. Therefore, contacting each facility individually remains the only viable option for collecting pertinent data. The authors did not have the resources to contact all 2,000 facilities. Thus, selected subsets of the total population that would provide a representative picture of all nonutilities were evaluated. Table 3 shows the proportion of the total nonutility capacity represented by several subsets. The subset of all U.S. nonutility facilities generating at least $150 \mathrm{MW}$, which includes 123 plants, was chosen. This subset represents $41,494 \mathrm{MW}$, or $56 \%$ of the total nonutility generating capacity. Using a list of plant names, addresses, facility contact persons, and phone numbers provided by EIA, the authors attempted to contact the facilities thus identified and inquire about their cooling systems.

A second data collection effort involved selected waste-to-energy plants, which constitute a different subset of the nonutility population. The Integrated Waste Services Association provided contact names and phone numbers for the six largest waste-to-energy operators, which collectively operate $58 \mathrm{U}$.S. plants generating about $2,200 \mathrm{MW}$. The information from these plants, which range in capacity from $4 \mathrm{MW}$ to $78 \mathrm{MW}$, gives an indication of cooling water use at the smaller end of the nonutility generating capacity spectrum. Each of the six companies was contacted. The interviews with company representatives were geared toward the future $\$ 316(\mathrm{~b})$ regulations and, therefore, were confined to determining the presence or absence of once-through cooling water intake structures. Information on the generating capacity of these facilities was obtained from EIA (1998a) and Rigo and Zannes (1998).

\section{Results}

\subsection{Facilities Generating At Least I50MW}

Table 4 lists the 123 nonutility facilities that generate at least $150 \mathrm{MW}$ of power, as well as the states in which they were located, MW generation rates, and cooling systems. Table 5 summarizes the number of facilities by type of cooling system. Survey responses could not be secured for 17 facilities representing $5,991 \mathrm{MW}$, because they could not be contacted (11) or did not reply (6). Percentages were thus assigned to the cooling systems used by 106 installations generating a total of 35,503 MW. Nearly half (48\%) employ closed-cycle cooling. Twenty-one percent do not utilize cooling processes. Thirteen percent are air-cooled. Eleven percent use once-through cooling. Six percent employ mixed-mode systems that include a once-through 
cooling element. One percent use other mixed mode approaches. In sum, the once-through share amounts to $17 \%$, representing $6,041 \mathrm{MW}$.

\subsection{Waste-to-Energy Facilities}

Table 6 lists the 58 waste-to-energy facilities operated by six companies, as well as the states in which they are located, MW generation rates, and reliance on once-through cooling or other, not specified, systems. Table 7 summarizes the number of installations by type of cooling system. Only 5 of 58 facilities employ once-through cooling. They represent $2,201 \mathrm{MW}$, or $11 \%$ of the total power generated by this subset of facilities.

\subsection{Geographic Distribution of Nonutilities Using Once-through Cooling}

Table 8 summarizes the number of facilities using once-through cooling by state. All but three of the 23 plants (18 larger nonutilities and 5 waste-to-energy installations) using once-through cooling are located in the eastern part of the country. About half of the facilities are located in New England or the Mid-Atlantic states. Seven other plants are situated in Gulf Coast states. All facilities, except for one located in Tennessee, operate in coastal states. Maryland and New York are the only states hosting once-through plants in both subsets. Louisiana, with four installations, is the only state hosting more than two once-through facilities. Three plants operate in Southeastern states and one facility each is located in the Midwest and Pacific Northwest.

\section{Discussion}

A relatively small portion of nonutility power sources utilize once-through cooling. A sizeable sample of the largest generators suggests that only about $17 \%$ use once-through cooling alone $(11 \%)$ or in conjunction with closed-cycle cooling in a mixed-mode system $(6 \%)$. This percentage share represents power generation of 6,041 MW.

Within the waste-to-energy subset of smaller nonutility facilities, about $11 \%$ use once-through cooling. No information is available for the other sectors of smaller nonutility facilities. The waste-to-energy industry is relatively new. Many of the facilities were constructed during the past decade. Nationally, the use of once-through cooling at newly constructed power plants has been declining since the mid-1980s. SAIC (1993) reports that only $26 \%$ of the cooling water intake flow at utility installations commissioned from 1986 to 1990 was utilized for oncethrough cooling systems. According to that study, no electric power facilities using oncethrough cooling went on line in 1991. In light of this national trend, the use of once-through cooling at waste-to-energy installations may be low because the facilities themselves are relatively new.

The total MW generated by once-through nonutilities can be estimated by adding the known capacities of the two subsets described above and an assumed percentage for the once-through nonutilities that have not actually been surveyed. As shown in Table 5, the larger nonutility subset represents a capacity of $6,041 \mathrm{MW}$. Table 7 indicates that the once-through waste-toenergy subset corresponds to a capacity of $252 \mathrm{MW}$. The summation of the two subsets yields a 
once-through capacity of 6,293 MW. As previously described, we received information on the cooling water use patterns from $37,704 \mathrm{MW}$ of nonutility capacity, while the overall nonutility capacity amounts to $74,021 \mathrm{MW}$. No cooling water usage information is available for the balance of $36,317 \mathrm{MW}$. For those facilities that provided information, the percentage using once-through cooling systems ranges from $11 \%$ to $17 \%$. The percentage of the remaining nonutility facilities using once-through cooling systems was estimated at $25 \%$ to provide a measure of conservatism in the analysis. The summation of the surveyed and estimated capacities yieids $15,372 \mathrm{MW}$ of capacity for facilities with once-through cooling.

The total power produced by nonutilities using once-through cooling $(15,372 \mathrm{MW})$ is equivalent to only about $6 \%$ of the $258,906 \mathrm{MW}$ generated by utilities using once-through cooling and may, from a national perspective, appear relatively insignificant. However, this appearance may not be accurate when the data are examined on a state-by-state basis. This situation is clearly illustrated in the State of Washington, where a small utility unit using once-through cooling produces just $44 \mathrm{MW}$. By comparison, the single nonutility once-through unit (a mixed-mode facility) generates $170 \mathrm{MW}$. In several other states, the nonutility once-through total is equivalent to more than $10 \%$ of the utility once-through total. In Maine, for example, the single once-through nonutility produces $298 \mathrm{MW}$, which is equivalent to $16 \%$ of the utility oncethrough total. The four Louisiana nonutility facilities using once-through cooling generate an amount of electricity equivalent to $14 \%$ of the utility once-through total. In Connecticut, the nonutility once-through total is equivalent to $12 \%$ of the utility total.

\section{Conclusions}

Only a relatively small portion of nonutility power sources utilize once-through cooling. The percentage of the generating capacity using once-through cooling ranges-from $11 \%$ to $1 \%$ in the two subsets of the nonutility population that were studied. A conservative estimate of the total once-through nonutility power generation yields $15,372 \mathrm{MW}$, which is equivalent to only $6 \%$ of the utility capacity employing once-through cooling. Potential impacts of future $\$ 316(b)$ regulations, which are expected to affect once-through cooling facilities in particular, may, from a national perspective, not be as significant for the nonutility sector because relatively few nonutilities use once-through cooling. However, this assessment may change when applied to individual states. These conclusions are based on the current distribution of utility and nonutility generating capacity. Should the proportion of nonutility capacity increase significantly in the future, these conclusions would need to be revisited.

\section{Acknowledgments}

Mr. Veil's and Dr. Puder's work was supported by the U.S. Department of Energy, Office of Fossil Energy and Office of Policy and International Affairs under Contract W-3 1-109-ENG-38. The authors acknowledge the assistance of Robert Schnapp of the Energy Information Administration, Maria Zannes of the Integrated Waste Services Association, and numerous personnel who responded at nonutility facilities.

\section{References}


EEL, 1996, Environmental Directory of US Powerplants, Edison Electric Institute, Washington, D.C.

EIA, 1998a, Electric Power Annual 1997, Volume II, U.S. Department of Energy, Energy Information Administration, DOE/ELA-0348(97)/2, October.

EIA, 1998b, Electric Power Anmual 1997, Volume I, U.S. Department of Energy, Energy Information Administration, DOE/EIA-0348(97)/1, July.

EIA, 1998c, Inventory of Power Plants in the United States As of January 1, 1998, U.S.

Department of Energy, Energy Information Administration, DOE/EIA-0095(98), December.

Rigo, G., and M. Zannes, 1998, The 1997-1998 IWSA Waste-To-Energy Directory of United States Facilities, Integrated Wastes Services Association, Washington, D.C.

SAIC, 1993, Cooling Water Use for Selected U.S. Industries and Summary of Selected EPA Regional and State Section 316(b) Activities, draft background paper 2, prepared for the U.S. Environmental Protection Agency by the Science Applications International Corporation, Falls Church, VA, December 13.

Veil, J.A., 1999, Potential Impacts of $316(\mathrm{~b})$ Regulatory Controls on Economics, Electricity Reliability, and the Environment, in proceedings of the Power Generation Impacts on Aquatic Resources Conference, sponsored by the Electric Power Research Institute and the U.S. Department of Energy, April 12-15, 1999, Atlanta, GA. 
Table I - Distribution of Types of Cooling Systems Used by Installed U.S. Steam-Electric Utilities

\begin{tabular}{|l|l|l|}
\hline Type of Cooling System & Capacity (MW) & Percentage of Total \\
\hline Once-through & 258,906 & 44 \\
\hline Cooling Tower & 206,605 & 35 \\
\hline Cooling Lake/Pond & 85,502 & 15 \\
\hline Combination & 24,070 & 4 \\
\hline Mixed-mode & 8,464 & 1 \\
\hline Other & 49 & 0 \\
\hline Total & 583,596 & 100 \\
\hline
\end{tabular}

Source: EEI (1996)

Table 2 - Distribution of Cooling Water Source Used by Installed U.S. Steam-Electric Utilities

\begin{tabular}{|l|l|l|}
\hline Cooling Water Source & Capacity (MW) & Percentage of Total \\
\hline River & 272,176 & 47 \\
\hline Lake & 160,419 & 27 \\
\hline Estuary & 62,210 & 11 \\
\hline Ocean & 49,705 & 9 \\
\hline Other & 39,819 & 7 \\
\hline Total & 584,329 & 100 \\
\hline
\end{tabular}

Source: EEI (1996) 
Table 3 - Generating Capacities Represented by Selected Populations of U.S. Nonutilities

\begin{tabular}{|l|l|l|l|}
\hline Population & $\begin{array}{l}\text { Number of } \\
\text { Facilities }\end{array}$ & Combined Capacity (MW) & Percentage of Total Capacity \\
\hline All & 1,983 & 74,021 & 100 \\
\hline $\begin{array}{l}\text { All facilities } \\
2100 \mathrm{MW}\end{array}$ & $2 \mathrm{Il}$ & 51,868 & 70 \\
\hline $\begin{array}{l}\text { All facilities } \\
2125 \mathrm{MW}\end{array}$ & 155 & 45,778 & 62 \\
\hline $\begin{array}{l}\text { All facilities } \\
2150 \mathrm{MW}\end{array}$ & 123 & 41,494 & 56 \\
\hline $\begin{array}{l}\text { All facilities } \\
2175 \mathrm{MW}\end{array}$ & 108 & 39105 & 53 \\
\hline $\begin{array}{l}\text { All facilities } \\
2200 \mathrm{MW}\end{array}$ & 95 & 36,674 & 50 \\
\hline
\end{tabular}

Source: Based on data from EIA (1998c) 
Table 4 - Cooling Systems Used at Nonutility Plants Generating $\geq 150 \mathrm{MW}$

\begin{tabular}{|c|c|c|c|}
\hline Facility Name & State & Capacit (MW) & Type of Cooling System \\
\hline Gcorgia-Pacific Corp & $\mathrm{AR}$ & 156.5 & Closed-cycle \\
\hline Pine Bluff Cogeneration Center & AR & 252.5 & Closed-cycle \\
\hline Nordic Power of South Point I & $\mathrm{AZ}$ & 233.2 & Unable to contact \\
\hline AES Placerita Incorporated & $\mathrm{CA}$ & 150 & Closed-cycle \\
\hline Collieville & $\mathrm{CA}$ & 253 & Unable to contact \\
\hline Crockett Cogeneration Project & $\overline{\mathrm{CA}}$ & $2+7.4$ & Closed-cycle \\
\hline Kem River Cogeneration Company & $\mathrm{CA}$ & 300 & No cooling system used \\
\hline Midway Sunset Cogeneration Company & CA & 234 & No cooling system used \\
\hline Sycamore Cogeneration Company & $\mathrm{CA}$ & 300 & No cooling system used \\
\hline Altamont Pass Windplant & $\overline{\mathrm{CA}}$ & 312.8 & Unable to contact \\
\hline Watson Cogeneration Company & $\mathrm{CA}$ & 398 & Closed-cycle \\
\hline Thermo Cogen Partnership L/P a Delaware L/P & $\mathrm{CO}$ & $\mathrm{I} 63$ & Unable to contact \\
\hline AES Thames, Incorporated & CT & 213.9 & Once-through \\
\hline Bridgeport Energy & $\mathrm{CT}$ & 520 & Once-through \\
\hline Auburndale Power Partners, Limited Partnership & FL & 192.8 & Closed-cycle \\
\hline Cedar Bay Generating Company $\mathrm{L} / \mathrm{P}$ & $\mathrm{FL}$ & 285 & Closed-cycle \\
\hline Indiantown Cogeneration Facility & F & 330 & Closed-cycle \\
\hline Mulberry Cogeneration Facility & FL & 153 & Closed-cycle \\
\hline Hardee Power Station & FL & 383.5 & No cooling system used \\
\hline Mid-Georgia Cogen & GA & 363.1 & Closed-cycle \\
\hline Union Camp Corporation - Savannah & GA & 192.5 & Mixed (once-through/closed-cycle) \\
\hline Hartwell Energy Limited Partnership & $\mathrm{GA}$ & 360 & No cooling system used \\
\hline Tenaska Georgia Generation Facility & GA & 306.8 & No cooling system used \\
\hline AES Barbers Point, Incorporated & $\mathrm{H}$ & 204 & Closed-cycle \\
\hline Kalaeola Cogeneration Plant & HI & 299.5 & Closed-cycle \\
\hline Cedar Rapids & IA & 155 & Closed-cycle \\
\hline Decatur & $\mathbb{L}$ & 261 & No information provided \\
\hline Bethlehem Steel Burns Harbor Plant & IN & $177 . \overline{7}$ & Once-through \\
\hline Georgia Gulf Corporation-Plaquemine Division & LA & 306 & Closed-cycle \\
\hline Nelson Industrial Steam Company & $\overline{\mathrm{LA}}$ & 200 & Closed-cycle \\
\hline Power and Utilities & LA & 587 & Once-through \\
\hline PPG - Riverside & LA & 159 & Once-through \\
\hline PPG- Powerhouse C & $\overline{\mathrm{LA}}$ & 357.8 & Once-through \\
\hline Sidney A. MurTay, Jr. Hydroelectric Station & LA & 192 & No cooling system used \\
\hline Taft Plant Union Carbide Corporation & $\overline{\mathrm{LA}}$ & 297.3 & Once-through \\
\hline Bellingham Cogeneration Facility & MA & 430.2 & Air-cooled \\
\hline Masspower & $\mathrm{MA}$ & 246 & Air-cooled \\
\hline Bershire Power & $\mathrm{MA}$ & 272 & Closed-cycle \\
\hline Milford Power Limited Partnership & MA & 178.1 & Closed-cycle \\
\hline Millennium Power & $\mathrm{MA}$ & 360 & Closed-cycle \\
\hline Dighton Power Associates & $\mathrm{MA}$ & 200 & Mixed (closed-cycle/air-cooled) \\
\hline Androscoggin Cogeneration Center & $\overline{\mathrm{MA}}$ & 163.7 & No cooling system used \\
\hline Island End Cogeneration Project & $\mathrm{MA}$ & 235 & Unable to contact \\
\hline AES Warior Run Cogeneration Facility & $\mathrm{MD}$ & 200 & Closed-cycle \\
\hline Panda Brandywine, L/P & $\mathrm{MD}$ & 288.9 & Closed-cycle \\
\hline
\end{tabular}




\begin{tabular}{|c|c|c|c|}
\hline Facility Name & State & Capacity (MW) & Type of Cooling System \\
\hline Bethlelem Sieel Sparrows Point & $\mathrm{MD}$ & 170 & Once-through \\
\hline Maine Independence Station & ME & 520 & Closed-cycle \\
\hline Great Northem Paper & ME & 297.5 & Once-through \\
\hline Michigan Power Limiled Partnership & MI & 154.1 & Closed-cycle \\
\hline Midland Cogeneration Venture & $\mathrm{MI}$ & 1849.5 & No cooling system used \\
\hline Rouge Powerhouse \#1 & $\mathrm{MI}$ & $3+5$ & Unable to contact \\
\hline Coltage Grove Cogeneration Facility & MN & 315 & Closed-cycle \\
\hline LTV Steel Mining Company-Schroeder & $\overline{\mathrm{MN}}$ & 202.5 & No information provided \\
\hline Caledonia Power Facility & MS & 290 & Air-cooled \\
\hline Batesville Generation Facility & $\overline{M S}$ & 801 & Closed-cycle \\
\hline Red Hills Generating Facility & MS & 513.8 & Closed-cycle \\
\hline Fulton Power Facility & MS & 290 & No cooling system used \\
\hline New Albany Power Facility & MS & 290 & No cooling system used \\
\hline Westmoreland - LG\&E Partners Roanoke Valley I & $\mathrm{NC}$ & 182.3 & No cooling system used \\
\hline Plymouth, NC & $\mathrm{NC}$ & 161.5 & Once-through \\
\hline Panda-Rosemary Limited Partnership & $\mathrm{NC}$ & 180 & Unable to contact \\
\hline Linden Cogen Plant & $\mathrm{NJ}$ & 761.6 & Air-cooled \\
\hline Sayreville Cogeneration Facility & $\mathrm{NJ}$ & 430.2 & Air-cooled \\
\hline Bayonne Cogen Plant & $\mathrm{NJ}$ & 191.6 & Closed-cycle \\
\hline Camden Cogen L.P. & $\mathrm{NJ}$ & 157 & Closed-cycle \\
\hline Eagle Point Cogeneration & $\mathrm{NJ}$ & 225 & Closed-cycle \\
\hline Lakewood Cogeneration, L/P & $\mathrm{NJ}$ & 238.5 & Closed-cycle \\
\hline Logan Generating Plant & $\mathrm{NJ}$ & 230 & Closed-cycle \\
\hline Chambers Cogeneration Limited Partnership & $\mathrm{NJ}$ & $285 \mid$ & No cooling system used \\
\hline Cobisa-Person Limited Partnership & NM & 150 & No cooling system used \\
\hline El Dorado Energy & NV & 632 & Air-cooled \\
\hline Nevada Sun-Peak Project & NV & 210 & Closed-cycle \\
\hline Saranac Facility & NY & 285.6 & Air-cooled \\
\hline Kodak Park Site & $\overline{N Y}$ & 206.9 & Closed-cycle \\
\hline Lockport Energy Assoc L/P Lockport Cogen Facility & NY & 209.6 & Closed-cycle \\
\hline Selkirk Cogen Partners, LP & NY & 357.6 & Closed-cycle \\
\hline Sithe/Independence Station & NY & 1305.5 & Closed-cycle \\
\hline Brooklyn Navy Yard Cogeneration Partners, L.P. & $\mathrm{NY}$ & 336.6 & Once-through \\
\hline Summit Pumped Storage Hydroelectric Project & $\mathrm{OH}$ & 1500 & Unable to contact \\
\hline AES Shady Point, Incorporated & OK & 350 & Closed-cycle \\
\hline Hermiston Generating Plant & OR & 621.2 & Closed-cycle \\
\hline Grays Ferry Cogeneration Partnership & PA & 192.6 & Once-through \\
\hline Tiverton Power Associates-Limited Partnership & RI & 272.9 & Air-cooled \\
\hline Ocean State Power & $\mathrm{RI}$ & $254.4 \mathrm{C}$ & Closed-cycle \\
\hline Ocean State Power II & $\mathrm{RI}$ & $25 \overline{4.4}$ & Closed-cycle \\
\hline Tenn Eastman Div, a Div of Eastman Chemical Co & IN & 188.9 & Mixed (once-through/closed-cycle) \\
\hline Brownsville Peaking Power Plant & TN & 290 & No cooling system used \\
\hline AES Deepwater, Incorporated & TX & $184 \mathrm{C}$ & Closed-cycle \\
\hline Encogen One & $\mathrm{TX}$ & 266 & Air-cooled \\
\hline Baytown Turbine Generator Project & $\mathrm{TX}$ & $2 \sqrt{2}$ & Closed-cycle \\
\hline C.R Wing Cogeneration Plant & $\mathrm{TX}$ & 224.5 & Closed-cycle \\
\hline Clear Lake Cogeneration Limited & $\mathrm{TX}$ & 377 & Closed-cycle \\
\hline
\end{tabular}




\begin{tabular}{|c|c|c|c|}
\hline \begin{tabular}{|c|} 
Facility Name \\
\end{tabular} & State & Capacity (MW) & Type of Cooling System \\
\hline Exxon Company USA-Baytown PP3/PP4 & TX & 225.5 & Closed-cycle \\
\hline Formosa Utility Venture, Limited & $\overline{\mathrm{TX}}$ & 652.2 & Closed-cycle \\
\hline Mustang Station & $\mathrm{TX}$ & 510 & Ciosed-cycle \\
\hline Oyster Creek Unit VIII & TX & 498 & Closed-cycle \\
\hline Tenaska Frontier Generation Station & TX & $5+9.6$ & Closed-cycle \\
\hline Tenaska III Texas Partners & TX & 250 & Closed-cycle \\
\hline Tenaska IV Texas Partners Ltd (Clebume Cogen) & $\overline{\mathrm{TX}}$ & 282.6 & Closed-cycle \\
\hline Shell Deer Park & $\mathrm{TX}$ & 255 & Mixed (closed-cycle/air-cooled) \\
\hline The Dow Chemical Company Texas Operations & TX & 1500.2 & Mixed (once-through/closed-cycle) \\
\hline Bayou Cogeneration Plant & TX & 300 & No cooling system used \\
\hline Beaumont Refinery & $\mathrm{TX}$ & 227.3 & No cooling system used \\
\hline Black Hawk Station & $\mathrm{TX}$ & 253.8 & No cooling system used \\
\hline Sweeny Cogeneration Facility & $\mathrm{TX}$ & 345 & No cooling system used \\
\hline Power Station $\$ 4$ & TX & 191.1 & No information provided \\
\hline Pasadena Power Plant & TX & 260.9 & No information provided \\
\hline Houston Chemical Complex Battleground Site & $\mathrm{TX}$ & 200 & No information provided \\
\hline Sandow & $\mathrm{TX}$ & 363 & Once-through \\
\hline CoGen Lyondell, Incorporated & TX & 564 & Unable to contact \\
\hline Cogenron, Incorporated & TX & 450 & Unable to contact \\
\hline Doswell Combined Cycle Facility & VA & 742.4 & Air-cooled \\
\hline Gordonsville Energy L.P. & VA & 300.4 & Air-cooled \\
\hline Cogentrix of Richmond, Incorporated & VA & 270 & Closed-cycle \\
\hline Commonwealth Atlantic Limited Partnership & VA & 388.9 & No cooling system used \\
\hline SEI Birchwood Power Facility & $\overline{\mathrm{VA}}$ & 240 & No information provided \\
\hline Franklin Fine Paper Division & VA & 155.6 & Once-through \\
\hline Hopewell Cogeneration & VA & 399 & Unable to contact \\
\hline Tenaska Washington Partners, L.P & WA & 245.7 & Closed-cycle \\
\hline Encogen NW & WA & 170.2 & Mixed (once-through/closed-cycle) \\
\hline March Point Cogeneration Company & WA & 167 & No cooling system used \\
\hline Whitesvater Cogeneration Facility & WI & $3 \overline{15}$ & Closed-cycle \\
\hline De Pere Energy Center & $\overline{\mathrm{W}}$ & 187.3 & No cooling system used \\
\hline Total -123 facilities & & $41,493.7$ & \\
\hline
\end{tabular}


Table 5 - Summary of Cooling Systems Used at Nonutility Plants Generating $\geq 150 \mathrm{MW}$ (from Table 4)

\begin{tabular}{|l|l|l|l|l|}
\hline $\begin{array}{l}\text { Type of Cooling } \\
\text { System }\end{array}$ & $\begin{array}{l}\text { Number of } \\
\text { Facilities }\end{array}$ & $\begin{array}{l}\text { Capacity } \\
\text { (MW) }\end{array}$ & $\begin{array}{l}\text { Percentage of } \\
\text { Total }\end{array}$ & $\begin{array}{l}\text { Percentage of Total } \\
\text { That Responded }^{3}\end{array}$ \\
\hline Once-through & 14 & 3,990 & 10 & 11 \\
\hline Closed-cycle & 53 & 16,904 & 41 & 48 \\
\hline Air-cooled & 11 & 4,657 & 11 & 13 \\
\hline $\begin{array}{l}\text { Mixed-mode } \\
\text { (includes some once- } \\
\text { through) }\end{array}$ & 4 & 2,051 & 5 & 6 \\
\hline $\begin{array}{l}\text { Mixed-mode } \\
\text { (includes no once- } \\
\text { through) }\end{array}$ & 2 & 455 & 1 & 1 \\
\hline $\begin{array}{l}\text { No cooling system } \\
\text { used }\end{array}$ & 22 & 7,496 & 18 & 21 \\
\hline $\begin{array}{l}\text { Unable to contact/no } \\
\text { information provided }\end{array}$ & 17 & 5,991 & 14 & -- \\
\hline Total & 123 & 41,494 & 100 & 100 \\
\hline
\end{tabular}

a The total that responded represents the overall total minus the 17 facilities in the "unable to contact/no information provided" category. The reduced total reflects a power generation of $35,503 \mathrm{MW}$. 
Table 6 - Cooling Systems Used at Selected Waste-to-Energy Facilities

\begin{tabular}{|c|c|c|}
\hline Facility Name & State & \begin{tabular}{|l|l|} 
MW & Once-Through Cooling Used \\
\end{tabular} \\
\hline Monicnay Power Corporation Long Beach SERRF & CA & $38 \mathrm{No}$ \\
\hline Ogden Martin Systems of Stanislaus, Inc. & $\mathrm{CA}$ & 22 No \\
\hline American Ref-Fuel Company of Southeastern Conneclicut & CT & \begin{tabular}{l|l}
17 & No \\
\end{tabular} \\
\hline Ogden Martin Systems of Bristol, Inc. & CT & 16) No \\
\hline ggden Martin Systems of Connecticut, Inc. & CT & 68 No \\
\hline Ogden Martin Projects of Wallingford L.P. & CT & II No \\
\hline \begin{tabular}{|l|} 
Wheelabrator Bridgeport RESCO \\
\end{tabular} & $\overline{C T}$ & 67 No \\
\hline Wheelabrator River Systems of Lisbon & CT & 15 No \\
\hline Montenay Power Corporation Dade County Resource Recovery Facility & $\mathrm{F}$ & $75 \sqrt{\mathrm{No}}$ \\
\hline Ogden Martin Systems of Hillsborough, Inc. & FL & 29 No \\
\hline Ogden Martin Systems of Lake, Inc. & FL & 16 No \\
\hline Ogden Martin Systems of Pasco, Inc. & FL & $31 /$ No \\
\hline Ogden Martin of Lee, Inc. & FL & $40 / \mathrm{No}^{2}$ \\
\hline Westinghouse Electric Corporation Bay County Energy Systems, Inc. & FL & 12 No \\
\hline Wheelabrator McKay Bay & F & 20 No \\
\hline Wheelabrator North Broward & FL & $\begin{array}{lll}66 & \text { No } \\
\end{array}$ \\
\hline Wheelabrator Pinellas & $\mathrm{FL}$ & $75 \widehat{\mathrm{No}}$ \\
\hline Wheelabrator South Broward & FL & 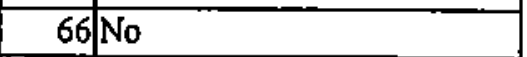 \\
\hline Ogden Martin Honolulu Resource Recovery Venture & $\mathrm{HI}$ & 57 No \\
\hline Foster Wheeler Robbins Resource Recovery Facility & $\pi$ & $\begin{array}{lll}\text { No } \\
50\end{array}$ \\
\hline Ogden Martin Systems of Indianapolis & IN & 7 No \\
\hline American Ref-Fuel Company of SEMASS, LP & $\mathrm{MA}$ & $78 /$ No \\
\hline Ogden Martin Systems of Haverhill, Inc. & MA. & \begin{tabular}{l|l|l}
46 & No \\
\end{tabular} \\
\hline Ogden Martin Haverhill Lawrence RDF Facility & $\overline{\mathrm{MA}}$ & $18 \sqrt{\mathrm{No}}$ \\
\hline Wheelabrator Massachuselts Refusetech & MA & \begin{tabular}{l|l} 
No \\
\end{tabular} \\
\hline Wheelabrator Millbury & MA & $45 \sqrt{\text { No }}$ \\
\hline Wheelabrator Saugus RESCO & MA & 54 Yes \\
\hline Ogden Martin Systems of Montgomery, Inc. & $\mathrm{MD}$ & $68 \sqrt{\mathrm{No}}$ \\
\hline Wheelabrator Baltimore RESCO & $M D$ & $60 \mid$ Yes \\
\hline Ogden Martin Systems Michigan Waste-to-Energy, Inc. & MI & $65 \sqrt{\mathrm{No}}$ \\
\hline Ogden Martin Systems of Kent, Inc. & MI & 18 No \\
\hline Ogden Martin Hennepin Energy Resource Company, L.P. & $\mathrm{MN}$ & $38 /$ No \\
\hline Wheelabrator Claremont RFD $\# 2$ & $\mathrm{NH}$ & 4 No \\
\hline Wheelabrator Concord & $\mathrm{NH}$ & 15 No \\
\hline American Ref-Fuel Company of Essex County & $\mathrm{NJ}$ & $68 \sqrt{\text { No }}$ \\
\hline Foster Wheeler Camden Resource Recovery Facility & $\overline{\mathrm{NJ}}$ & $34 \sqrt{\text { No }}$ \\
\hline Ogden Martin Warren Energy Resource Company, L.P. & $\mathrm{NJ}$ & \begin{tabular}{l|l|l}
13 & No \\
\end{tabular} \\
\hline Ogden Martin Systems of Union, Inc. & NJ & \begin{tabular}{l|l} 
No \\
\end{tabular} \\
\hline Wheelabrator Gloucester & NJ & $1 4 \longdiv { \text { Yes } }$ \\
\hline American Ref-Fuel Company of Hempstead & $\mathrm{NY}$ & 72 No \\
\hline American Ref Fuel Company of Niagara, L.P. & $\overline{N Y}$ & 50 No \\
\hline Foster Wheeler Washington County Facility & NY & $64 \mid$ Yes \\
\hline Ogden Martin Systems of Babylon, Inc. & $\mathrm{NX}$ & 17 No \\
\hline Ogden Martin Systems of Huntington, Inc. & $\overline{N Y}$ & 25 No \\
\hline Ogden Martin Systems of Onondaga, Inc. & NY & $\begin{array}{lll}40 & \text { No } \\
\end{array}$ \\
\hline
\end{tabular}




\begin{tabular}{|l|l|l|l|}
\hline \multicolumn{1}{|c|}{ Facility Name } & State & MW & Once-Through Cooling Used \\
\hline Westinghouse Electric Corporation Dutchess County Resource & NY & 12 & No \\
\hline Wheelabrator Westchester RESCO & NY & 60 & Yes \\
\hline Ogden Martin Systems of Tusa, Inc. & OK & 18 & No \\
\hline Ogden Martin Systems of Marion, Inc. & OR & 13 & No \\
\hline American Ref-Fuel Delaware Valley Resource Recovery Facility & PA & 80 & No \\
\hline Montenay Power Corporation Energy Resources of Montgomery & PA & 32 & No \\
\hline Ogden Martin Systems of Lancaster, Inc. & PA & 36 & No \\
\hline Westinghouse Electric Corporation York County Resource Energy, Inc. & PA & 36 & No \\
\hline Wheelabrator Falls & PA & 53 & No \\
\hline Foster Wheeler Charleston Facility & SC & 13 & No \\
\hline Ogden Martin Systems of Alexandria/Arlington, Inc. & VA & 22 & No \\
\hline Ogden Martin Systems of Fairfax, Inc. & VA & 80 & No \\
\hline Wheelabrator Spokane & WA & 26 & No \\
\hline Total & & 2201 & \\
\hline
\end{tabular}

Table 7 - Summary of Cooling Systems Used at Selected Waste-to-Energy Facilities from Table 6

\begin{tabular}{|l|l|l|l|}
\hline $\begin{array}{l}\text { Type of Cooling } \\
\text { System }\end{array}$ & Number of Facilities & Capacity (MW) & Percentage of Total \\
\hline Once-through & 5 & 252 & 11 \\
\hline All others & 53 & 1,808 & 89 \\
\hline Total & 58 & 2,201 & 100 \\
\hline
\end{tabular}


Table 8 - Occurrence of Nonutility Plants Using Once-Through Cooling in Two Subsets by State

\begin{tabular}{|c|c|c|c|c|}
\hline \multirow[t]{2}{*}{ State } & \multicolumn{2}{|c|}{ Facilities Generating $\geq 150 \mathrm{MW}$} & \multicolumn{2}{|c|}{ Selected Waste-to-Energy Facilities } \\
\hline & Total Facilities & $\begin{array}{l}\text { Facilities Using } \\
\text { Once-Through } \\
\text { Cooling } \\
\end{array}$ & Total Facilities & \begin{tabular}{|l|} 
Facilities Using \\
Once-Through \\
Cooling \\
\end{tabular} \\
\hline$\overline{A R}$ & 2 & 0 & 0 & 0 \\
\hline $\mathrm{AZ}$ & 1 & 0 & 0 & 0 \\
\hline $\mathrm{CA}$ & 8 & 0 & 2 & 0 \\
\hline $\mathrm{CO}$ & 1 & 0 & 0 & $\overline{0}$ \\
\hline$\overline{\mathrm{CT}}$ & 2 & 2 & 6 & 0 \\
\hline $\mathrm{FL}$ & 5 & 0 & 10 & 0 \\
\hline GA & 4 & 1 & 0 & $\overline{0}$ \\
\hline $\mathrm{HI}$ & 2 & 0 & 1 & 0 \\
\hline IA & 1 & 0 & 0 & 0 \\
\hline$\tilde{\mathrm{IL}}$ & 1 & $\overline{0}$ & 1 & 0 \\
\hline $\mathrm{IN}$ & 1 & 1 & 1 & 0 \\
\hline LA & 7 & 4 & 0 & 0 \\
\hline MA & 8 & 0 & 6 & 1 \\
\hline $\mathrm{MD}$ & 3 & I & 2 & 1 \\
\hline ME & 2 & 1 & 0 & 0 \\
\hline MI & 3 & 0 & 2 & 0 \\
\hline $\mathrm{MN}$ & 2 & 0 & I & 0 \\
\hline MS & 5 & 0 & 0 & 0 \\
\hline NH & 0 & 0 & 2 & 0 \\
\hline $\mathrm{NC}$ & 3 & 1 & 0 & 0 \\
\hline $\mathrm{NJ}$ & 8 & 0 & 5 & 1 \\
\hline NM & I & $\overline{0}$ & 0 & 0 \\
\hline NV & 2 & 0 & 0 & 0 \\
\hline $\mathrm{NY}$ & 6 & 1 & 8 & 2 \\
\hline $\mathrm{OH}$ & 1 & 0 & 0 & 0 \\
\hline $\mathrm{OK}$ & 1 & 0 & 1 & 0 \\
\hline
\end{tabular}




\begin{tabular}{|l|l|l|l|l|}
\hline State & \multicolumn{3}{|l|}{ Facilities Generating $2150 \mathrm{MW}$} & \multicolumn{2}{l|}{ Selected Waste-to-Energy Facilities } \\
\hline & Total Facilities & $\begin{array}{l}\text { Facilities Using } \\
\text { Once-Through } \\
\text { Cooling }\end{array}$ & Total Facilities & $\begin{array}{l}\text { Facilities Using } \\
\text { Once-Through } \\
\text { Cooling }\end{array}$ \\
\hline OR & 1 & 0 & 1 & 0 \\
\hline PA & 1 & 1 & 5 & 0 \\
\hline RI & 3 & 0 & 0 & 0 \\
\hline SC & 0 & 0 & 1 & 0 \\
\hline TN & 2 & 1 & 0 & 0 \\
\hline TX & 24 & 2 & 0 & 0 \\
\hline VA & 7 & 1 & 2 & 0 \\
\hline WA & 3 & I & 1 & 0 \\
\hline WI & 2 & 0 & 0 & 0 \\
\hline Total & 123 & 18 & 58 & 5 \\
\hline
\end{tabular}

